Natieli Alves da Silva

\title{
Aplicação da Teoria Quântica de Átomos em Moléculas em estudos de complexação, aromaticidade e excitação eletrônica
}

Tese apresentada ao Instituto de Química de São Carlos da Universidade de São Paulo como parte dos requisitos para a obtenção do título de Doutora em Ciências.

Área de concentração: Físico-Química

Orientador: Prof. Dr. Roberto Luiz A. Haiduke

\section{Exemplar revisado}

O exemplar original encontra-se em acervo reservado na Biblioteca do

IQSC-USP

São Carlos 
Aos meus pais, José e Orlete, a minha irmã, Taciane, e ao meu amor, Evandro, pelo apoio incondicional e por iluminarem a minha vida. 


\section{AGRADECIMENTOS}

Aos meus pais, José e Orlete, pelo amor, carinho, compreensão, incentivo e por me ajudarem a realizar meus sonhos. Muito obrigada por terem feito sempre o possível e o impossível por mim. Vocês são grandes exemplos e me inspiram a dar cada passo na minha vida. É inconcebível e aterrador pensar em um mundo em que vocês não estejam ao meu lado. Afinal, como viver sem os anjos que guardam o meu caminho? Eu os amo infinitamente, pai e mãe!

A minha irmã, Taciane, que sempre esteve ao meu lado em todos os momentos desde que nasci. Obrigada por desbravar os caminhos para que eu pudesse passar sem grandes danos! Obrigada por ser esta pessoa maravilhosa que procuro seguir na esperança de adquirir as suas melhores qualidades! Mas como você tem muitas delas, esta tarefa se tornou impossível! Afinal, como você consegue comprender escritos tão inintelígiveis e explicar de forma tão acessível? Ahhhhhh, e que capacidade de dominar todo um lugar com a sua fala e interpretar tudo com um viés literário e filosófico tão único...

Ao meu namorado, Evandro, que é o vento fresco que vem do mar, a luz que me aquece e o abraço que me conforta. Muito obrigada por todo o apoio e carinho que tem me dado desde que lhe conheci e por transformar os meus dias mais nublados em dias ensolarados e os meus dias mais tristes em dias alegres. Você é uma pessoa muito especial e admirável e que me faz querer sempre ser a melhor versão de mim mesma. Afinal, tenho ao meu lado um professor incrível, que se preocupa com o outro e que possui a força e a serenidade para encarar todos os desafios.

Ao meu orientador, professor Haiduke, por todos os ensinamentos que me transmitiu ao longo destes anos, pelas aulas instigantes, pela paciência em explicar um dado assunto de diferentes ângulos e maneiras possíveis e, sobretudo, por ser um professor excepcional e um cientista admirável. Sei que nunca poderei agradecê-lo o suficiente por tudo o que aprendi, por isso, apenas desejo que sejas muito feliz e faça a diferença na vida de inúmeras outras pessoas como fez na minha.

Ao meu ex-orientador, professor Valderi, que fez nascer em mim o amor pela físicoquímica e que possui uma contribuição gigantesca na minha formação acadêmica. Você foi e sempre será um grande exemplo de profissional para mim. Espero que muitos outros alunos tenham o privilégio e saibam aproveitar o presente que é encontrá-lo logo no início do 
caminho.

Aos professores do ensino médio, Edson e Márcia, que me mostraram como a química pode ser fascinante e, por meio de suas aulas, me direcionaram para esta área e me conduziram no início da minha jornada. Obrigada por terem me ajudado a dar o primeiro passo na minha trajetória acadêmica, auxiliando-me na preparação para o vestibular.

Às amigas do Paraná, Adriana, Naihara, Stephany, Paula e Maristela pelos inúmeros momentos de alegria, de descontração e de trabalho compartilhados. Obrigada por deixarem a minha vida mais leve, serena e, por simplesmente estarem presentes. Saibam que fui muito abençoada em ter a amizade de vocês.

A minha família de São Carlos, Aline, Amanda, Aldinéia, Eriosvaldo, Estevão, Fernando G., Gabriel, Guilherme, Genisson, Júlia, Luiz, Ronaldo, Régis e Wagner por terem tornado os anos de mestrado e doutorado bem mais significativos, as horas de trabalho mais agradáveis e os momentos de lazer inesquecíveis. Ao término deste ciclo, escrevo estes agradecimentos com um certo saudosismo e revivendo tantas lembranças de cada um de vocês que marcaram este período. Obrigada por tudo, meus queridos amigos/irmãos!

A todas as pessoas que de forma direta ou indireta contribuíram para a realização deste trabalho.

À Capes pela concessão da bolsa de doutorado e à FAPESP pelos recursos computacionais. 
"Ah! Tudo era compreensivel e contudo triste, embora belo. Ninguém sabia nada. Vivia-se, corria-se por esse mundo afora, ou cavalgava-se pelas florestas e muitas coisas nos olhavam de maneira tão desafiadora, com promessas, ou provocando saudades: uma estrela durante a noite, uma campânula azul, um lago de um verde como o dos canaviais, o olho de um ser humano [...]; às vezes parecia que algo nunca visto, porém longamente desejado, ia de repente acontecer, como se fosse cair um véu diante de todos; depois passava tudo isso. Nada acontecia, o enigma não era decifrado, o encanto secreto não se desfazia; finalmente ficavase velho [...], sempre sem saber nada, esperando e à espreita."

Hermann Hesse, Narciso e Goldmund. 


\section{RESUMO}

Da SILVA, N. A. Aplicação da Teoria Quântica de Átomos em Moléculas em estudos de complexação, aromaticidade e excitação eletrônica. 2020. 142. Tese - Instituto de Química de São Carlos, Universidade de São Paulo, São Carlos, 2020.

O modelo Carga - Fluxo de Carga - Fluxo de Dipolo (CCFDF), em termos dos multipolos da Teoria Quântica de Átomos em Moléculas (QTAIM), foi empregado para investigar as variações das intensidades de infravermelho durante a dimerização de ácidos carboxílicos, na coordenação de carbonilas e grupos cianeto a centros metálicos diversos e durante a excitação eletrônica de moléculas diatômicas simples $(\mathrm{AlH}, \mathrm{AlF}, \mathrm{AlCl}, \mathrm{AlBr}, \mathrm{BF}$, $\mathrm{BCl}, \mathrm{BBr}$ e CS). A intensificação das bandas de estiramento $\mathrm{OH}$ durante o processo de dimerização e as variações de intensidade das bandas de estiramento dos ligantes complexados a centros metálicos podem ser justificadas, principalmente, pelo termo cruzado entre carga e fluxo de carga. Por sua vez, a interpretação da variação da intensidade de infravermelho durante a excitação eletrônica requer a consideração de três contribuições, carga e seus termos cruzados com fluxo de carga e fluxo de dipolo. Além disto, a dimerização de ácidos carboxílicos é acompanhada por uma diminuição da densidade eletrônica nos BCPs das ligações $\mathrm{O}-\mathrm{H}$, resultando no enfraquecimento e redução do caráter covalente destas ligações. No que se refere à coordenação do ligante carbonil a metais de transição, observa-se também, na maioria dos casos, o enfraquecimento da ligação CO. Certos dados topológicos analisados, advindos da QTAIM, estão em grande concordância com as expectativas em termos da capacidade de retrodoação dos centros metálicos investigados. Por sua vez, as excitações verticais geralmente ocasionam reduções na densidade eletrônica dos pontos críticos de ligação nas moléculas diatômicas estudadas quando comparadas com os respectivos estados fundamentais. A transição eletrônica $\sigma \rightarrow \pi^{*}$ provoca ainda uma inversão no sentido do vetor de momento de dipolo para o estado tripleto das moléculas $\mathrm{BF}, \mathrm{BCl}, \mathrm{BBr}$ e CS. Estas mudanças de sentido deste vetor são causadas pela diminuição expressiva das contribuições de polarização atômica do boro e do carbono durante a excitação. Por fim, baseando-se apenas nas propriedades dos substratos iniciais, as curvaturas da densidade eletrônica nos pontos críticos dos anéis aromáticos (RCP) e dos núcleos de carbono, as densidades de energia cinética e potencial no RCP, juntamente com a energia intra-atômica dos carbonos, são apresentadas como descritores promissores para predizer a reatividade e a regiosseletividade em reações de substituição eletrofílica aromática.

Palavras-chave: intensidade de infravermelho, complexação, reatividade e regiosseletividade. 


\begin{abstract}
Da SILVA, N. A. Application of the Quantum Theory of Atoms in Molecules in studies of complexation, aromaticity, and electronic excitation. 2020. 142. Thesis - São Carlos Institute of Chemistry, University of São Paulo, São Carlos, 2020.
\end{abstract}

The Charge - Charge flux - Dipole flux (CCFDF) model, in terms of multipoles from the Quantum Atom Theory of Molecules (QTAIM), was employed to investigate the variations in infrared intensities during the dimerization of carboxylic acids, in the coordination of carbonyls and cyanide groups to different metallic centers and during the electronic excitation of simple diatomic molecules $(\mathrm{AlH}, \mathrm{AlF}, \mathrm{AlCl}, \mathrm{AlBr}, \mathrm{BF}, \mathrm{BCl}, \mathrm{BBr}$, and $\mathrm{CS}$ ). The intensification of $\mathrm{OH}$ stretching bands during the dimerization process and the variations in the intensity of the stretching bands of ligands complexed to metal centers can be justified, mainly, by the cross term between charge and charge flux. On the other hand, the interpretation of infrared intensity variations during electronic excitation requires the consideration of three contributions, charge and its cross - terms with charge flux and dipole flux. In addition, the dimerization of carboxylic acids is accompanied by a decrease in the electron density at the BCPs of O-H bonds, resulting in the weakening and covalent character decrements of these bonds. Regarding the coordination of carbonyl to transition metals, the $\mathrm{CO}$ bond weakening is also observed in most cases. Certain topological data analyzed, obtained from QTAIM, are in great agreement with the expectations regarding the backbonding capacity of the investigated metal centers. On the other hand, vertical excitations usually provoke electron density decrements at the critical points of the studied diatomic molecules when compared with the respective fundamental states. The $\sigma \rightarrow \pi^{*}$ electronic transition also causes dipole moment inversions for the triplet state of $\mathrm{BF}, \mathrm{BCl}, \mathrm{BBr}$, and $\mathrm{CS}$ molecules. The direction changes of this vector can be justified by the significant decrease observed in the atomic polarization contributions of boron and carbon during excitation. Finally, based only on the properties of initial substrates, the electron density curvatures at the critical points of aromatic rings (RCP) and of carbon nuclei, the densities of kinetic and potential energy at the RCP, along with the intra-atomic energy of carbons, are presented as promising descriptors to predict the reactivity and regioselectivity in aromatic electrophilic substitution reactions.

Keywords: infrared intensities, complexation, reactivity and regioselectivity. 


\section{LISTA DE FIGURAS}

Figura 1 - Pontos críticos envolvendo somente átomos de carbono da molécula de cubano, $\mathrm{C}_{8} \mathrm{H}_{8}$, em que os pontos críticos nucleares são representados pelas esferas azuis, os pontos críticos de ligação pelas esferas verdes, os pontos críticos dos anéis pelas esferas vermelhas e o ponto crítico de sela pela esfera amarela

Figura 2 - Forma dos átomos da molécula hidreto de lítio $(\mathrm{LiH})$ segundo as delimitações das superfícies de fluxo zero dadas pela partição QTAIM

Figura 3 - Estrutura dos dímeros de ácido carboxílicos plotada com o software Molden (version 5.3) (SCHAFTENAAR e NOORDIK, 2000), em que $\mathrm{X}$ e $\mathrm{X}^{\prime}$ referem a $\mathrm{H}, \mathrm{CH}_{3}, \mathrm{~F}, \mathrm{Cl}$ ou $\mathrm{Br}$ (note o anel formado após a dimerização)

Figura 4 - Correlação entre a energia de dimerização, $E_{H B}$, e a média das distâncias de

ligação O-H, $\langle r(O \ldots H)\rangle$, segundo cálculos B2PLYP/aug-cc-pVTZ 42

Figura 5 - Correlação entre as densidades eletrônicas nos pontos críticos das ligações de hidrogênio e de hidroxilas, respectivamente $\rho_{B C P}(\mathrm{O} \ldots \mathrm{H})$ e $\rho_{B C P}(\mathrm{O}-\mathrm{H})$, de acordo com cálculos B2PLYP/aug-cc-pVTZ (em u. a.)

Figura 6 - Correlação entre a energia interatômica das ligações de hidrogênio, $E_{\text {Inter }}(\mathrm{O} \ldots H)$, segundo cálculos B3LYP/aug-cc-pVTZ, e a densidade eletrônica nos seus respectivos BCPs, $\rho_{B C P}(\mathrm{O} \ldots \mathrm{H})$, de acordo com resultados B2PLYP/aug-cc-pVTZ (em u. a.)

Figura 7 - Correlação entre as energias de interação interatômica da ligação de hidrogênio, $E_{\text {Inter }}(\mathrm{O} \ldots \mathrm{H})$, obtidas a partir de cálculos B3LYP/aug-cc-pVTZ, e o Laplaciano da densidade eletrônica nos respectivos BCPs, $\nabla^{2} \rho_{B C P}(\mathrm{O} \ldots \mathrm{H})$, segundo cálculos B2PLYP/aug-cc-pVTZ (em u. a.)

Figura 8 - Correlação entre a energia de dimerização, $E_{H B}$, e o Laplaciano da densidade eletrônica nos pontos críticos dos anéis, $\nabla^{2} \rho_{R C P}$, para dímeros de ácidos carboxílicos segundo cálculos B2PLYP/aug-cc-pVTZ (em u. a.)

Figura 9 - Variação da intensidade de infravermelho dos modos de estiramento O-H durante a dimerização, $\Delta A$, em função da soma da contribuição de carga, $\Delta A^{C}$, e seu termos cruzados com fluxo de carga e fluxo de dipolo, $\Delta A^{C \times C F}$ e $\Delta A^{C \times D F}$, segundo cálculos B2PLYP/aug-ccpVTZ $\left(\mathrm{em} \mathrm{km} \mathrm{mol}{ }^{-1}\right)$

Figura 10 - Contribuições para as variações das intensidades de infravermelho totais dos modos de estiramento $\mathrm{O}-\mathrm{H}$ durante a dimerização segundo modelo CCFDF/QTAIM: (1) Cargas e seus termos cruzados com os fluxos de carga e de dipolos atômicos, $\Delta A^{C}+\Delta A^{C \times C F}+$ $\triangle A^{C \times D F}$, e (2) Fluxo de carga e fluxo de dipolo e o termo cruzado entre estes dois termos, $\Delta A^{C F}+\Delta A^{D F}+\Delta A^{C F \times D F}\left(\mathrm{em} \mathrm{km} \mathrm{mol}^{-1}\right)$ 56

Figura 11 - Variação da intensidade de infravermelho dos modos de estiramento O-H durante a dimerização, $\Delta A$, em função da contribuição cruzada entre a carga e o fluxo de carga, $\triangle A^{C \times C F}$, segundo cálculos B2PLYP/aug-cc-pVTZ (em km mol ${ }^{-1}$ )

Figura 12 - Sobreposição entre os orbitais HOMO e LUMO do metal e do ligante e o efeito da ligação $\pi$ sobre o desdobramento do campo ligante

Figura 13 - Geometrias de equilíbrio dos complexos de coordenação estudados plotadas com o software Molden (version 5.3) (SCHAFTENAAR e NOORDIK, 2000) 
Figura 14 - Variação da intensidade de infravermelho total dos modos de estiramento $\mathrm{CO}$ e $\mathrm{CN}$ durante a complexação por unidade de ligante, $\Delta A$, em função da contribuição cruzada entre a carga e o fluxo de carga, $\Delta A^{C \times C F}\left(\mathrm{em} \mathrm{km} \mathrm{mol}^{-1}\right)$ 75

Figura 15 - Correlação entre as médias da densidade de energia total, $H_{B C P}$, e da densidade eletrônica, $\rho_{B C P}$, nos pontos críticos das ligações entre o metal e o ligante (em u. a.) 77

Figura 16 - Representação esquemática dos estados de spin eletrônico singleto e tripleto durante uma excitação hipotética do tipo HOMO - LUMO

Figura 17 - Diagrama de Jablonski ilustrando um processo fotoquímico ___ 88

Figura 18 - Energias de transição vertical e adiabática __ 90

Figura 19 - Variação da intensidade de infravermelho dos modos de estiramento das moléculas $\mathrm{AlH}, \mathrm{AlF}, \mathrm{AlCl}, \mathrm{AlBr}, \mathrm{BF}, \mathrm{BCl}, \mathrm{BBr}$ durante a excitação eletrônica, $\Delta A$, em função da soma da contribuição de carga, $\Delta A^{C}$, e seus termos cruzados com fluxo de carga e fluxo de dipolo, $\Delta A^{C \times C F}$ e $\Delta A^{C \times D F}\left(\mathrm{em} \mathrm{km} \mathrm{mol}^{-1}\right)$

Figura 20 - Diagrama de coordenada de reação para a substituição eletrofílica do benzeno119 Figura 21 - Autovetores das curvaturas da densidade eletrônica $\left(\lambda_{2}\right.$ e $\left.\lambda_{3}\right)$ para o benzeno, $\mathrm{C}_{6} \mathrm{H}_{6}$ plotados com o software Molden (version 5.3) (SCHAFTENAAR e NOORDIK, 2000)

Figura 22 - Relação entre as curvaturas da densidade eletrônica $\Delta \lambda_{1}$ e $\Delta \lambda_{3}$ no ponto crítico do anel, RCP (em u. a.) 128

Figura 23 - Correlação entre as curvaturas da densidade eletrônica $\Delta \lambda_{2}$ e $\Delta \lambda_{3}$ no ponto crítico do anel, RCP (em u. a.)

Figura 24 - Correlação entre as variações de densidades de energia cinética $\left(\triangle G_{R C P}\right)$ e potencial $\left(\Delta V_{R C P}\right)$ no ponto crítico do anel em relação ao benzeno (em u. a.) 130

Figura 25 - Correlação entre a variação da segunda curvatura da densidade eletrônica, $\Delta \lambda_{2}$, (em u. a.) e o parâmetro empírico de efeito de ressonância $\left(\sigma_{R}\right)$ de Taft (os substituintes ativantes e desativantes estão destacados em azul e vermelho, respectivamente)

Figura 26 - Correlação entre a variação da densidade de energia potencial em relação ao benzeno, $\Delta V_{R C P}$, (em u. a.) e o parâmetro empírico de efeito de ressonância $\left(\sigma_{R}\right)$ de Taft (os substituintes ativantes e desativantes estão destacados em azul e vermelho, respectivamente) 134

Figura 27 - Correlação entre a curvatura da densidade eletrônica perpendicular ao núcleo carbono orto em relação ao benzeno, $\Delta \lambda_{\text {perp }}$, (em u. a.) e o parâmetro empírico de ressonância de Taft $\left(\sigma_{R}\right)$ (os substituintes ativantes e desativantes estão destacados em azul, respectivamente) 135

Figura 28 - Correlação entre a curvatura da densidade eletrônica perpendicular ao núcleo carbono para em relação ao benzeno, $\Delta \lambda_{\text {perp }}$, (em u. a.) e o parâmetro empírico de ressonância de Taft $\left(\sigma_{R}\right)$ (os substituintes ativantes e desativantes estão destacados em azul, respectivamente) 135 


\section{LISTA DE TABELAS}

Tabela 1 - Energias das ligações de hidrogênio $\left(E_{H B}\right)$ durante a dimerização de ácidos carboxílicos obtidas em cálculos B2PLYP/aug-cc-pVTZ ( $\mathrm{kcal} \mathrm{mol}^{-1}$ )

Tabela 2 - Propriedades QTAIM nos pontos críticos das ligações de hidrogênio calculadas em nível B2PLYP/aug-cc-pVTZ (em u. a.)

Tabela 3 - Variações nas propriedades topológicas QTAIM nos pontos críticos de ligação O$\mathrm{H}$ de dímeros de ácidos carboxílicos em relação aos monômeros isolados em suas geometrias de equilíbrio calculadas em nível B2PLYP/aug-cc-pVTZ (em u. a.)

Tabela 4 - Energias de interação IQA $\left(E_{\text {Inter }}\right)$ juntamente com os componentes clássico $\left(V_{c l}\right) \mathrm{e}$ de troca-correlação $\left(V_{X C}\right)$ para o par de átomos diretamente envolvido na ligação de hidrogênio $(\mathrm{O} . . . \mathrm{H})$ de dímeros de ácidos carboxílicos calculados em nível B3LYP/aug-ccpVTZ (em u. a.)

Tabela 5 - Variações na energia de interação IQA $\left(\Delta E_{\text {Inter }}\right)$ e nos componentes clássico $\left(\Delta V_{c l}\right)$ e de troca-correlação $\left(\Delta V_{X C}\right)$ das ligações $\mathrm{O}-\mathrm{H}$ durante a dimerização em nível de cálculo B3LYP/aug-cc-pVTZ (em u. a.)

Tabela 6 - Laplaciano e as três curvaturas da densidade eletrônica nos pontos críticos dos anéis (RCP), $\nabla^{2} \rho_{R C P}$ e $\lambda$, respectivamente, dos dímeros de ácidos carboxílicos, obtidos a partir de cálculos B2PL-YP/aug-cc-pVTZ (em u. a.)

Tabela 7 - Magnitude do momento de dipolo molecular, $|\vec{\mu}|$, para os monômeros e dímeros bidentados (em D) 51

Tabela 8 - Frequências vibracionais $\left(\mathrm{cm}^{-1}\right)$ e intensidades fundamentais de infravermelho $\left(\mathrm{km} \mathrm{mol}^{-1}\right)$ dos modos de estiramento O-H dos monômeros isolados, obtidas a partir do nível de cálculo B2PLYP/aug-cc-pVTZ e calculadas por meio do modelo CCFDF/QTAIM 52 Tabela 9 - Frequências vibracionais $\left(\mathrm{cm}^{-1}\right)$ e intensidades fundamentais de infravermelho $\left(\mathrm{km} \mathrm{mol}^{-1}\right)$ dos modos de estiramento O-H dos homo- e heterodímeros de ácidos carboxílicos, calculadas diretamente em nível de cálculo B2PLYP/aug-cc-pVTZ e obtidas por meio do modelo CCFDF/QTAIM

Tabela 10 - Variações das contribuições de carga, fluxo de carga, fluxo de dipolo e os termos cruzados entre estas quantidades durante a dimerização para a intensidade total, $A,\left(\mathrm{~km} \mathrm{~mol}^{-1}\right)$ dos modos de estiramento O-H segundo o modelo CCFDF/QTAIM

Tabela 11 - Variações nas cargas atômicas, obtidas a partir dos formalismos QTAIM e NPA, dos dímeros de ácidos carboxílicos em relação aos monômeros isolados em nível de cálculo B2PLYP/aug-cc-pVTZ (em u. a.)

Tabela 12 - Distâncias de ligação para os ligantes $\left(\mathrm{CO}\right.$ e $\left.\mathrm{CN}^{-}\right)$livres e nos complexos metálicos, obtidas em nível de cálculo B2PLYP/aug-cc-pVTZ, bem como os dados experimentais disponíveis (em $\AA$ ) 71

Tabela 13 - Frequências vibracionais $\left(\mathrm{cm}^{-1}\right)$ e intensidades fundamentais de infravermelho $\left(\mathrm{km} \mathrm{mol}^{-1}\right)$ dos modos de estiramento $\mathrm{CO}$ e $\mathrm{CN}$ dos ligantes livres e dos complexos metálicos, calculadas diretamente em nível de cálculo B2PLYP/aug-cc-pVTZ e obtidas por meio do modelo CCFDF/QTAIM

Tabela 14 - Variações das contribuições de carga, fluxo de carga, fluxo de dipolo e os termos cruzados entre estas quantidades durante a complexação por unidade de ligante para a 
intensidade total, $\mathrm{A},\left(\mathrm{km} \mathrm{mol}^{-1}\right)$ dos modos de estiramentos $\mathrm{CO}$ e $\mathrm{CN}$ segundo o modelo CCFDF/QTAIM

Tabela 15 - Médias das propriedades QTAIM nos pontos críticos das ligações entre o metal e o ligante calculadas em nível B2PLYP/aug-cc-pVTZ (em u. a.)

Tabela 16 - Variações das propriedades QTAIM nos pontos críticos das ligações $\mathrm{CO}$ e CN nos complexos de coordenação estudados em relação aos ligantes livres, calculadas em nível B2PLYP/aug-cc-pVTZ (em u. a.)

Tabela 17 - Médias das energias de interação IQA ( $\left.\left\langle E_{\text {Inter }}\right\rangle\right)$ juntamente com os componentes clássico $\left(V_{c l}\right)$ e de troca-correlação $\left(V_{X C}\right)$ para as ligações metal-carbono, carbono-oxigênio e carbono-nitrogênio dos ligantes livres e dos complexos de coordenação, calculadas em nível B2PLYP/aug-cc-pVTZ (em u. a.)

Tabela 18 - Valores médios das energias de interação IQA entre o metal e o ligante, assim como os seus componentes eletrostático e de troca-correlação, obtidos em nível de cálculo B2PLYP/aug-cc-pVTZ (em u. a.)

Tabela 19 - Cargas atômicas QTAIM dos complexos de coordenação estudados, bem como suas variações em relação aos ligantes livres (em $e$ )

Tabela 20 - Energias de excitação verticais $\left(\Delta E_{v e r t}\right)$ e adiabáticas $\left(\Delta E_{a d}\right)$ das moléculas $\mathrm{AlH}$, $\mathrm{AlF}, \mathrm{AlCl}, \mathrm{AlBr}, \mathrm{BF}, \mathrm{BCl}, \mathrm{BBr}$ e CS, calculadas com o nível de teoria PBE0/aug-cc-pVTZ $(\mathrm{em} \mathrm{eV})$

Tabela 21 - Componentes da energia atômica, segundo a Partição Virial, obtidas por meio de cálculos PBE0/aug-cc-pVTZ nas geometrias de equilíbrio de cada estado (em u. a.) 94

Tabela 22 - Distâncias de ligação para os estados fundamentais e excitados, obtidas a partir de cálculos PBE0/aug-cc-pVTZ, e resultados experimentais disponíveis (em $\AA$ )

Tabela 23 - Momentos de dipolo $(\vec{\mu})$ e seus componentes, polarização atômica $\left(\vec{\mu}_{A P}\right)$ e transferência de carga $\left(\vec{\mu}_{C T}\right)$, obtidos a partir de cálculos PBE0/aug-cc-pVTZ nas geometrias de equilíbrio do estado fundamental e excitados para as moléculas estudadas (em D)

Tabela 24 - Decomposição atômica do momento de dipolo $(\vec{\mu})$ dos sistemas $\mathrm{AlH}, \mathrm{AlF}, \mathrm{AlCl}$, $\mathrm{AlBr}, \mathrm{BF}, \mathrm{BCl}, \mathrm{BBr}$ e CS calculados com PBE0/aug-cc-pVTZ nas geometrias de equilíbrio de cada estado (As cargas estão em u. a. e os momentos de dipolo em $\mathrm{D}$ ) 101

Tabela 25 - Momentos de quadrupolo atômico QTAIM ao longo do eixo molecular de ligação $\left(Q_{z z}\right)$ obtidos em cálculos PBE0/aug-cc-pVTZ das moléculas $\mathrm{AlH}, \mathrm{AlF}, \mathrm{AlCl}, \mathrm{AlBr}$, $\mathrm{BF}, \mathrm{BCl}, \mathrm{BBr}$ e CS (em u. a.)

Tabela 26 - Propriedades topológicas QTAIM nos pontos críticos de ligação calculadas em nível PBE0/aug-cc-pVTZ para processos de excitação verticais e adiabáticos (em u. a.) ${ }^{\mathrm{a}}{ }_{\text {_ }} 107$

Tabela 27 - Frequências vibracionais harmônicas $(\omega)$, obtidas em cálculos PBE0/aug-ccpVTZ, e resultados experimentais $\left(\mathrm{em} \mathrm{cm}^{-1}\right)$

Tabela 28 - Contribuições para as intensidades fundamentais de infravermelho segundo análise CCFDF/QTAIM, assim como as obtidas em cálculos PBE0/aug-cc-pVTZ, e suas variações durante a excitação eletrônica $\left(\mathrm{em} \mathrm{km} \mathrm{mol}^{-1}\right)^{\mathrm{a}}$

Tabela 29 - Variações nos componentes $z z$ dos momentos de quadrupolo atômicos, $\Delta Q_{z z}$, do carbono de compostos aromáticos substituídos (ArY, em que Y é o substituinte) em relação ao benzeno (Ar), segundo cálculos B2PLYP/cc-pVQZ (em u. a.) 
Tabela 30 - Variações das propriedades topológicas densidade eletrônica $\left(\Delta \rho_{R C P}\right)$ e suas curvaturas $\left(\Delta \lambda_{1}, \Delta \lambda_{2}\right.$ e $\left.\Delta \lambda_{3}\right)$, Laplaciano da densidade eletrônica $\left(\Delta \nabla^{2} \rho_{R C P}\right)$, densidades de energia potencial e cinética $\left(\Delta V_{R C P}\right.$ e $\left.\Delta G_{R C P}\right)$, assim como a total $\left(\Delta H_{R C P}\right)$, no ponto crítico do anel aromático em relação ao benzeno (em u. a.)

Tabela 31 - Variações das curvaturas da densidade eletrônica perpendiculares ao anel aromático no atratores nucleares de átomos de carbonos, $\Delta \lambda_{\text {perp }}$, de acordo com cálculos B2PLYP/cc-VQZ (em u. a.) 131

Tabela 32 - Variações das energias intra-atômicas e de seus componentes (energia cinética eletrônica do átomo $\Omega, T(\Omega)$, energia de atração elétron-núcleo, $V_{e n}(\Omega)$, e energia de interação elétron-elétron, $V_{e e}(\Omega)$ ) durante a substituição do hidrogênio do benzeno (Ar) por diferentes substituintes (Y), obtidas em nível de cálculo B3LYP/cc-pVQZ (em u. a.) 136 


\section{LISTA DE ABREVIATURAS E SIGLAS}

AP - Polarização atômica (do inglês “atomic polarization”).

B2PLYP - Funcional de troca-correlação duplo híbrido com correção de perturbação de segunda ordem.

B3LYP - Funcional híbrido de três parâmetros Becke Lee-Yang-Parr (do inglês "Becke 3 parameter Lee-Yang-Parr functional”).

BCP - Ponto crítico de ligação (do inglês "Bond critical point").

BSSE - Erro de superposição das funções de base (do inglês "Basis set superposition error"). C - Carga.

CCFDF - Carga - Fluxo de Carga - Fluxo de Dipolo (do inglês "Charge - Charge flux e Dipole flux").

CCP - Ponto crítico de cela (do inglês "Cage critical point”).

CF - Fluxo de carga (do inglês "Charge flux").

CP - Ponto crítico (do inglês "Critical point").

CT - Transferência de carga (do inglês "Charge transfer").

DF - Fluxo de dipolo (do inglês "Dipole dipole").

DFT - Teoria do Funcional de densidade (do inglês “Density Functional Theory”).

Exp. - Experimental.

Freq. - Frequência vibracional.

$\mathbf{G}_{\mathbf{C P}}$ - Densidade de energia cinética no ponto crítico (do inglês "Kinetic energy density at the critical point").

GGA - Aproximação do gradiente generalizado (do inglês "Generalized gradiente approximation").

HB - Ligação de hidrogênio (do inglês "Hydrogen bond").

HCP - Densidade de energia total no ponto crítico (do inglês "Total energy density at the critical point").

HOMO - Orbital molecular ocupado de maior energia (do inglês "Highest occupied molecular orbital").

Int. - Intensidade de infravermelho.

IQA - Átomos Quânticos Interagentes (do inglês "Interacting Quantum Atoms").

LDA - Aproximação da densidade local (do inglês "Local density approximation"). 
LUMO - Orbital molecular desocupado de menor energia (do inglês "Lowest unccupied molecular orbital").

NCP - Ponto crítico nuclear (do inglês “nuclear critical point”).

NPA - Análise de População Natural.

NOX - Número de oxidação.

PBE0 - Funcional híbrido de troca e de correlação de correlação de Perdew-BurkeErnzerhof.

PBE - Funcional de Perdew-Burke-Ernzerhof.

PW91 - Funcional de Perdew e Wang 91.

QTAIM - Teoria Quântica de Átomos em Moléculas (do inglês "Quantum Theory of Atoms in Molecules").

RCP - Ponto crítico do anel (do inglês "ring critical point").

SEAr - Substituição eletrofílica aromática.

TD-DFT - Teoria do funcional de densidade dependente do tempo (do inglês "Timedependent density functional theory").

Teo. - Teórico.

TD-DFT - Teoria do Funcional de Densidade Dependente do Tempo (do inglês "TimeDependent Density Functional Theory")

$\mathbf{V}_{\mathbf{C P}}$ - Densidade de energia potencial no ponto crítico (do inglês "potential energy density at the critical point").

ZPE - Energia do ponto zero (do inglês "Zero point energy"). 


\section{SUMÁRIO}

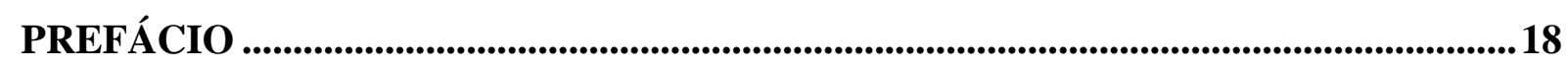

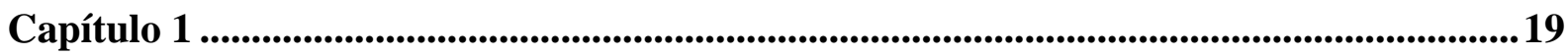

Introdução à Teoria Quântica de Átomos em Moléculas (QTAIM)..................................... 19

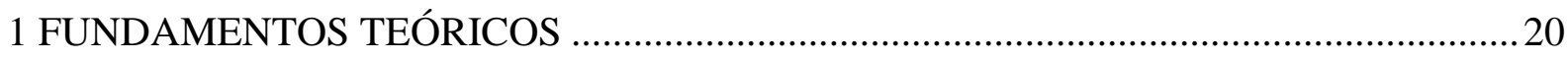

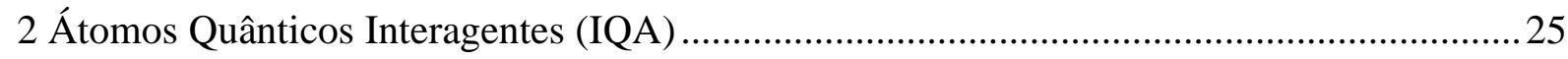

3 Modelo de partição Carga - Fluxo de Carga - Fluxo de Dipolo em termos dos multipolos da

Teoria Quântica de Átomos em Moléculas (CCFDF/QTAIM) .............................................26

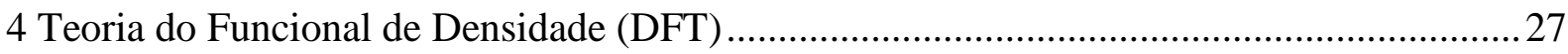

5 Teoria do Funcional de Densidade Dependente do Tempo (TD-DFT)...................................31

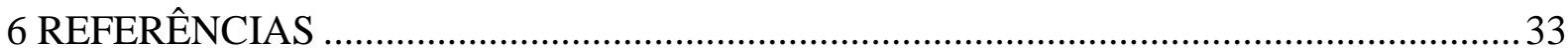

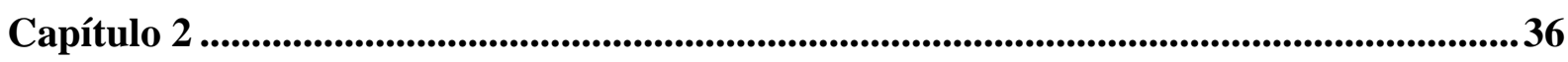

Análise das intensidades de infravermelho dos modos de estiramento da hidroxila de dímeros de ácidos carboxílicos por meio do modelo de Carga - Fluxo de carga - Fluxo

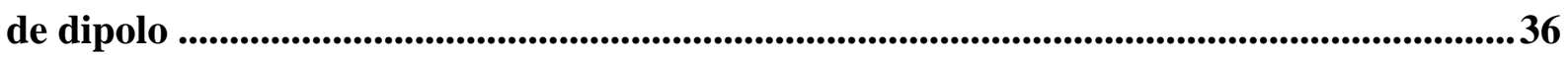

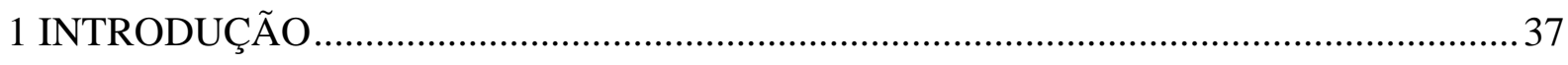

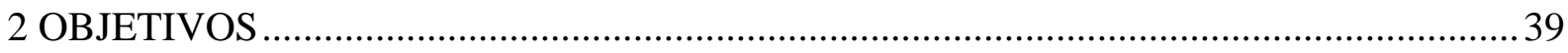

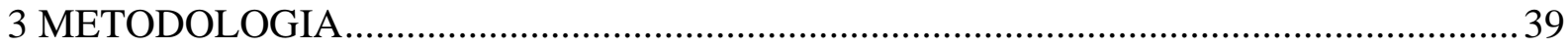

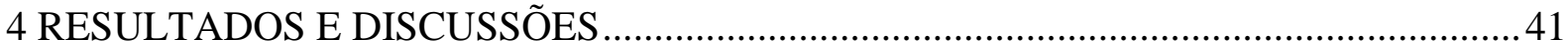

4.1 Propriedades energéticas e topológicas das ligações de hidrogênio.................................. 41

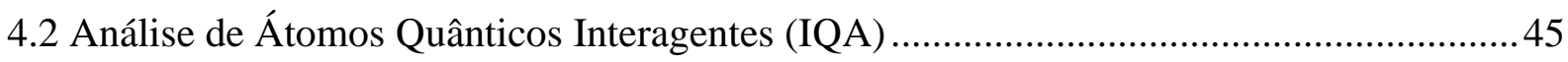

4.3 Momentos de dipolo e propriedades espectroscópicas dos monômeros e dímeros de ácidos

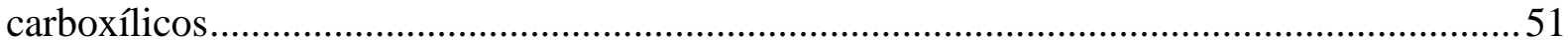

4.4 Análise das variações de carga atômicas durante a dimerização........................................57

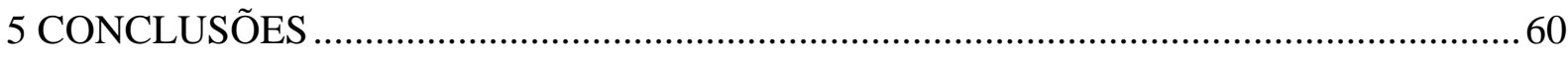

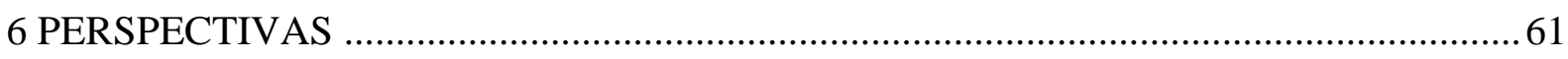

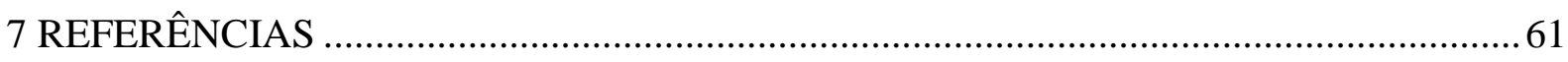

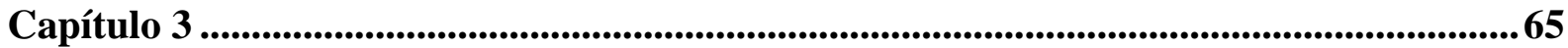

Análise de carga, fluxo de carga e fluxo de dipolo para as intensidades de infravermelho dos modos de estiramento $\mathrm{CO}$ em carbonilas metálicas .......................................................65

1 INTRODUÇÃ

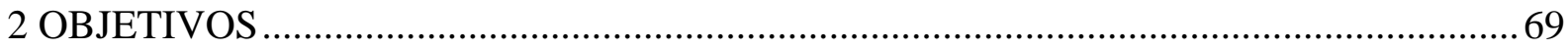

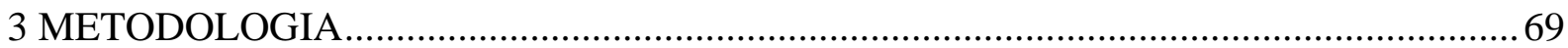




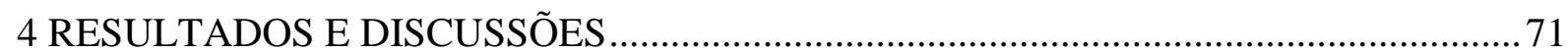

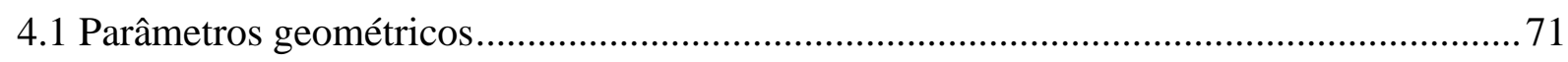

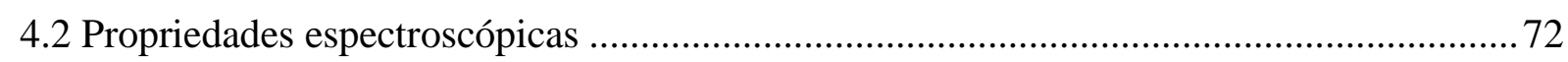

4.4 Análise de Átomos Quânticos Interagentes .................................................................... 78

4.5 Análise das variações de cargas atômicas durante a complexaçãa ................................... 80

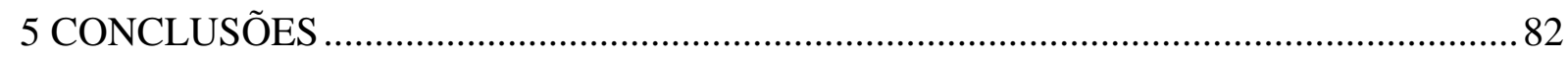

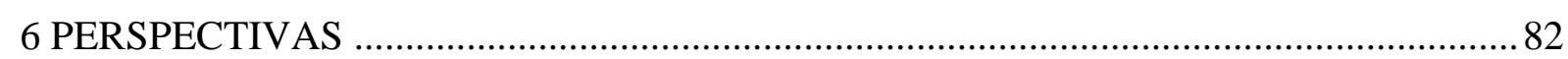

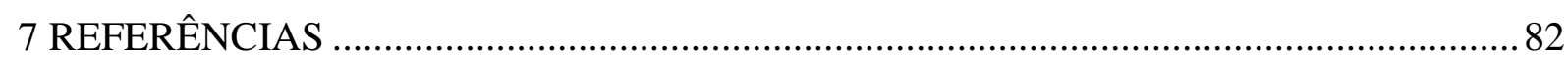

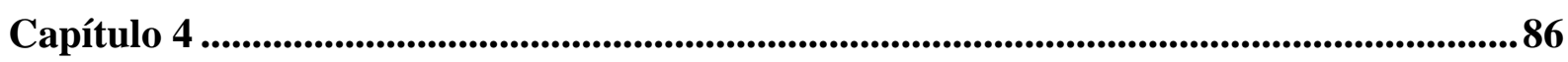

Análise das variações das intensidades de infravermelho durante a excitação eletrônica segundo o modelo de Carga - Fluxo de Carga - Fluxo de dipolo .............................................86

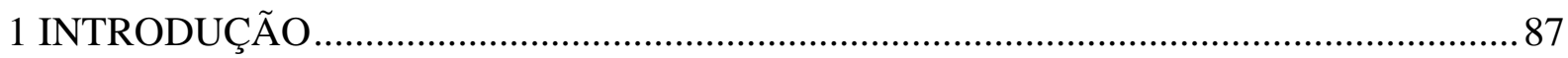

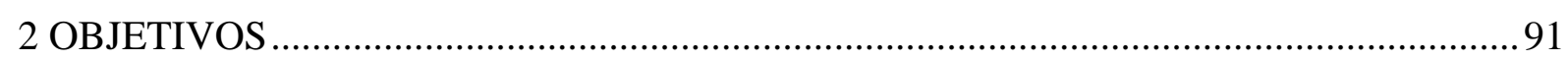

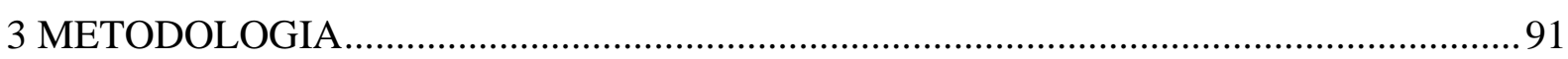

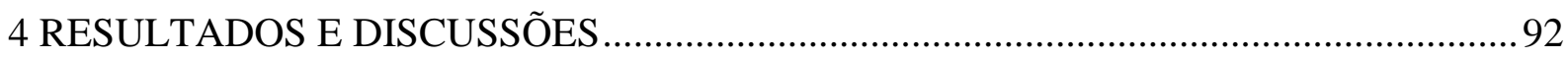

4.1 Energias de excitação vertical e adiabática ...................................................................... 92

4.2 Energias atômicas obtidas a partir da aproximação Virial .............................................. 94

4.3 Comprimentos de ligação nos estados fundamentais e excitados ....................................96

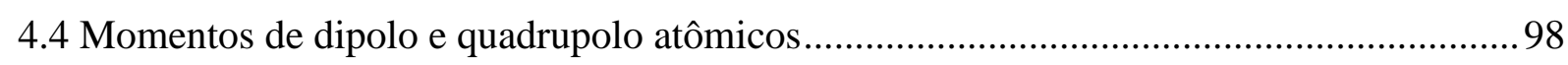

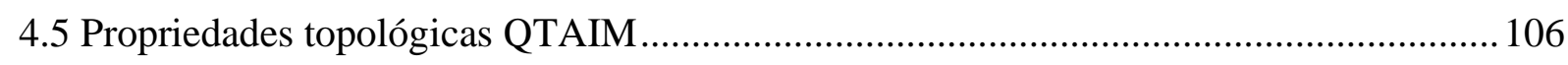

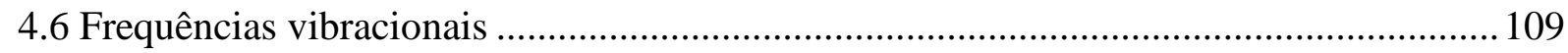

4.7 Análise de Carga - Fluxo de Carga - Fluxo de Dipolo das intensidades de infravermelho

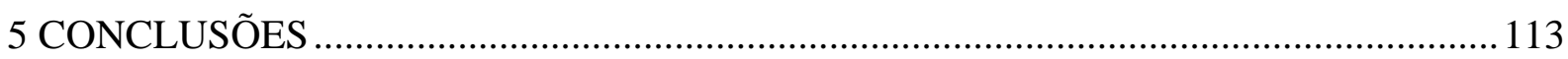

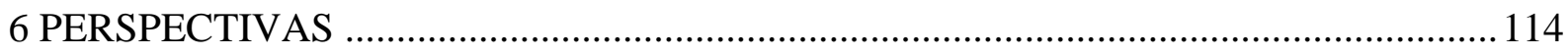

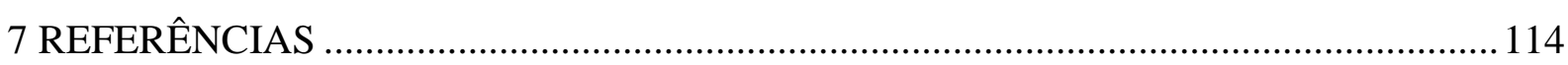

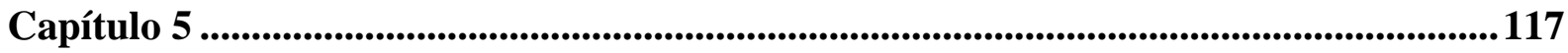

Propriedades topológicas da densidade eletrônica para a predição da reatividade e da regiosseletividade em substituições eletrofílicas aromáticas ............................................117

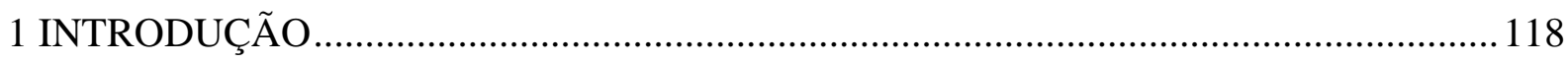

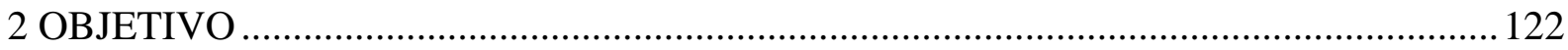

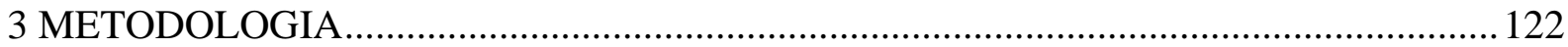

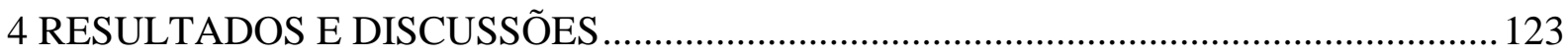


4.1 Efeitos dos substituintes nos momentos de quadrupolo dos átomos de carbono de anéis

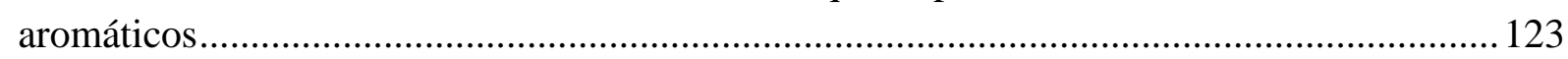

4.2 Propriedades topológicas QTAIM nos pontos críticos dos anéis aromáticos ...................126

4.3 Análise das variações de energia intra-atômica segundo a partição de Átomos Quânticos

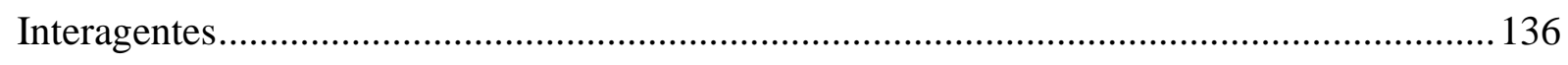

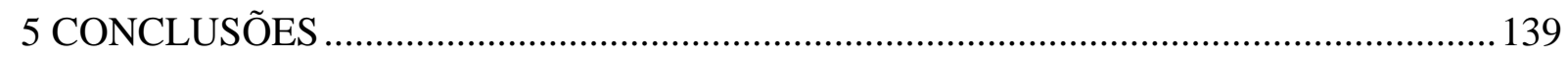

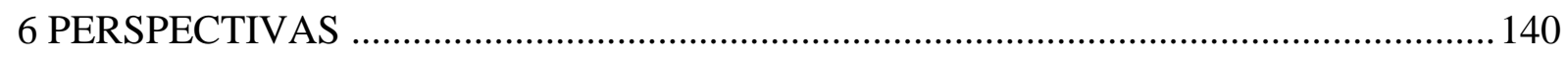

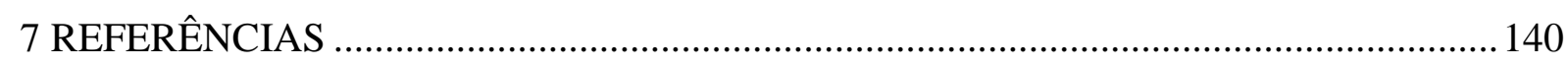

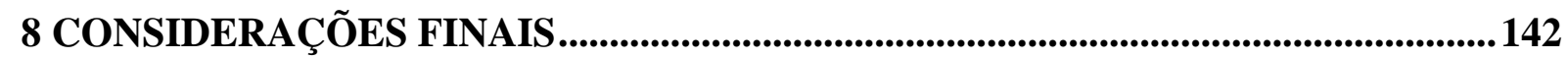




\section{PREFÁCIO}

Esta tese reúne as minhas principais contribuições em termos de aplicações da Teoria Quântica de Átomos em Moléculas (QTAIM), proposta por Bader (1989), em diferentes aspectos da química, como estudos de propriedades espectroscópicas, ligações de hidrogênio, complexos metálicos, estados excitados e reatividade/regiosseletividade de compostos aromáticos substituídos. Os fundamentos teóricos da QTAIM serão abordados no decorrer do capítulo 1, assim como o modelo de Carga - Fluxo de carga - Fluxo de Dipolo segundo os multipolos da Teoria Quântica de Átomos em Moléculas (CCFDF/QTAIM) (HAIDUKE e BRUNS, 2005), o qual é utilizado para a interpretação das intensidades vibracionais de infravermelho. Além disso, a partição energética de Átomos Quânticos Interagentes (IQA) (PENDÁS, BLANCO e FRANCISCO, 2005), que utiliza os critérios preconizados pela QTAIM para a definição dos subsistemas atômicos, e a Teoria do Funcional de Densidade, empregada na metodologia computacional, serão apresentadas de forma sucinta. No capítulo 2 aplicaremos o modelo de partição CCFDF/QTAIM para compreender a natureza das variações nas intensidades de infravermelho durante a formação de ligações de hidrogênio em dímeros de ácidos carboxílicos. No capítulo 3, investigaremos o efeito da complexação sobre as propriedades espectroscópicas das carbonilas metálicas segundo o mesmo modelo mencionado anteriormente. Além disso, no capítulo 4, avaliaremos como as partições QTAIM e de CCFDF explicam, respectivamente, as inversões no sentido do vetor de momento dipolar e o enfraquecimento ou fortalecimento de bandas vibracionais durante a excitação eletrônica de moléculas diatômicas simples. Por fim, mostraremos no capítulo 5 que as quantidades QTAIM e IQA podem ser utilizadas como alternativas para predizer a reatividade e a regiosseletividade em reações de substituição eletrofílica aromática baseando-se apenas em propriedades dos substratos iniciais. 
Capítulo 1

Introdução à Teoria Quântica de Átomos em Moléculas (QTAIM) 


\section{FUNDAMENTOS TEÓRICOS}

O arcabouço teórico-matemático fornecido pela Teoria Quântica de Átomos em Moléculas (QTAIM), proposta por Bader (1990), possibilita explicar conceitos químicos tradicionais com base na teoria quântica e, portanto, possui aplicações em diferentes campos que incluem, por exemplo, química de ligação, aromaticidade, reatividade, espectroscopia, fotofísica, estados excitados e análise conformacional (GARCÍA-REVILLA et al., 2018). Muitas propriedades de uma molécula, como a suscetibilidade diamagnética, o momento de dipolo, o campo elétrico e o gradiente do campo elétrico podem ser total ou parcialmente determinados por meio de uma análise da distribuição tridimensional da densidade eletrônica (MATTA e BOYD, 2007).

A QTAIM está fundamentada na partição inequívoca da densidade eletrônica $(\rho(\vec{r}))$ e no fato desta quantidade apresentar valores máximos nas posições dos núcleos permitindo a partição da molécula em regiões mononucleares separadas, os átomos. Esta divisão, baseada em critérios topológicos, satisfaz importantes teoremas da química quântica, como o Teorema Virial (BADER, 1990). Assim, a superfície que delimita um átomo em uma molécula é a superfície de fluxo zero, que satisfaz a condição particular:

$$
\nabla \rho(\vec{r}) \cdot \vec{n}(\vec{r})=0
$$

em que $\vec{n}$ é o vetor normal a esta superfície e $\nabla \rho(\vec{r})$ é o vetor gradiente da densidade eletrônica, para todos os pontos $\vec{r}$ da superfície. As linhas de caminho gradiente de átomos distintos (linhas que seguem as trajetórias ditadas pelos vetores $\nabla \rho(\vec{r})$ ) nunca se cruzam. Desta forma, tais linhas se tornam paralelas à superfície de fluxo zero (BADER, 1990).

A análise topológica da densidade eletrônica fornece informações sobre vários tipos de interações intra- e intermoleculares, sendo relevante para se caracterizar um dado sistema molecular. Um ponto no espaço no qual a primeira derivada da densidade eletrônica é nula é denominado ponto crítico, CP (MATTA e BOYD, 2007). Este ponto pode ser um mínimo local, um máximo local ou um ponto de sela. Uma maneira de distingui-los é por meio da segunda derivada da densidade eletrônica. As nove possibilidades da derivada segunda da densidade eletrônica formam a matriz Hessiana que, quando calculada no ponto crítico, pode ser expressa do seguinte modo (GRABOWSKI, 2011): 


$$
A\left(r_{C P}\right)=\left(\begin{array}{lll}
\frac{\partial^{2} \rho}{\partial x^{2}} & \frac{\partial^{2} \rho}{\partial x \partial y} & \frac{\partial^{2} \rho}{\partial x \partial z} \\
\frac{\partial^{2} \rho}{\partial y \partial x} & \frac{\partial^{2} \rho}{\partial y^{2}} & \frac{\partial^{2} \rho}{\partial y \partial z} \\
\frac{\partial^{2} \rho}{\partial z \partial x} & \frac{\partial^{2} \rho}{\partial z \partial y} & \frac{\partial^{2} \rho}{\partial z^{2}}
\end{array}\right)_{r=r_{C}}
$$

A matriz Hessiana pode ser diagonalizada mediante uma transformação unitária:

$$
\begin{aligned}
\Lambda\left(r_{C P}\right) & =\left(\begin{array}{ccc}
\frac{\partial^{2} \rho}{\partial x^{\prime 2}} & 0 & 0 \\
0 & \frac{\partial^{2} \rho}{\partial y^{\prime 2}} & 0 \\
0 & 0 & \frac{\partial^{2} \rho}{\partial z^{\prime 2}}
\end{array}\right)_{r \prime=r_{C}} \\
\Lambda\left(r_{C P}\right) & =\left(\begin{array}{ccc}
\lambda_{1} & 0 & 0 \\
0 & \lambda_{2} & 0 \\
0 & 0 & \lambda_{3}
\end{array}\right)
\end{aligned}
$$

na qual $\lambda_{1}, \lambda_{2}$ e $\lambda_{3}$ são as curvaturas da densidade eletrônica em relação aos eixos principais x', y' e z' (MATTA e BOYD, 2007; GRABOWSKI, 2011; BADER, 1990).

Os pontos críticos são classificados de acordo com o número de curvaturas da densidade eletrônica que são diferentes de zero e por meio da soma algébrica dos seus sinais, sendo que cada curvatura $\lambda$ contribui com \pm 1 dependendo se é positiva ou negativa (GRABOWSKI, 2011). Há quatro pontos críticos estáveis em uma molécula e cada um deles está associado a um elemento da estrutura química: $(3,-3)$ ponto crítico nuclear (do inglês "nuclear critical point" - NCP); $(3,-1)$ ponto crítico de ligação (do inglês "bond critical point" - BCP); $(3,+1)$ ponto crítico do anel (do inglês "ring critical point" - RCP) e $(3,+3)$ ponto crítico de sela (do inglês "cage critical point" - CCP), como ilustrados na Figura 1 (MATTA e BOYD, 2007). 
Figura 1 - Pontos críticos envolvendo somente átomos de carbono da molécula de cubano, $\mathrm{C}_{8} \mathrm{H}_{8}$, em que os pontos críticos nucleares são representados pelas esferas azuis, os pontos críticos de ligação pelas esferas verdes, os pontos críticos dos anéis pelas esferas vermelhas e o ponto crítico de sela pela esfera amarela

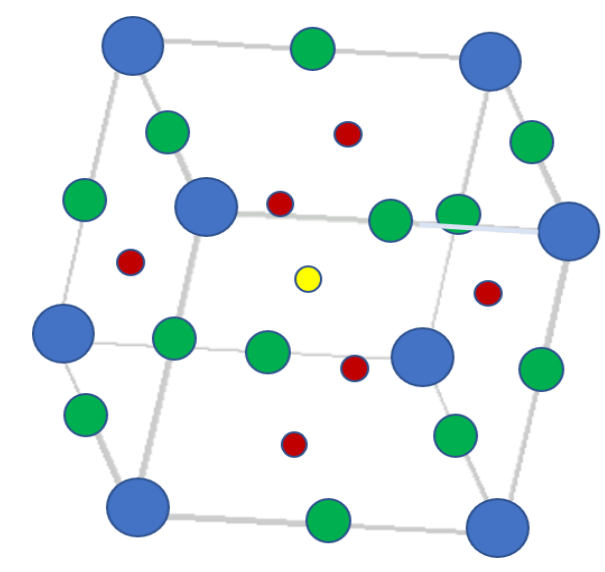

Fonte: Adaptado de MATTA, C. F. e BOYD, R. J. The Quantum Theory of Atoms in Molecules From Solid State to DNA and Drug Design, Weinheim: Wiley-Vch, 2007.

A soma das curvaturas $\lambda_{1}, \lambda_{2}$ e $\lambda_{3}$ fornece o Laplaciano da densidade eletrônica, $\nabla^{2} \rho(\vec{r})$, que é invariante frente à rotação do sistema de coordenadas (Eq. 5). Quando $\rho_{B C P}$ é grande, normalmente encontramos uma concentração de carga eletrônica no ponto crítico da ligação $\left(\nabla^{2} \rho_{B C P}>0\right)$. Por sua vez, quando $\rho_{B C P}$ é pequeno, observa-se uma tendência à depleção de carga eletrônica na região internuclear $\left(\nabla^{2} \rho_{B C P}<0\right)$ (MATTA e BOYD, 2007). Matematicamente,

$$
\nabla^{2} \rho(\vec{r})=\frac{\partial^{2} \rho}{\partial x^{\prime 2}}+\frac{\partial^{2} \rho}{\partial y^{\prime 2}}+\frac{\partial^{2} \rho}{\partial z^{\prime 2}}=\lambda_{1}+\lambda_{2}+\lambda_{3}
$$

O sinal do Laplaciano da densidade eletrônica em um ponto específico do espaço determina se a energia potencial negativa ou a energia cinética positiva está acima da razão virial por um valor de 2, visto que a relação entre o Laplaciano da densidade eletrônica e os componentes da densidade de energia, $H_{C P}$, é dada por (MATTA e BOYD, 2007; BADER, 1990):

$$
\frac{1}{4} \nabla^{2} \rho_{C P}=2 G_{C P}+V_{C P},
$$


na qual $G_{C P}$ é a densidade de energia cinética e $V_{C P}$ é a densidade de energia potencial local. A soma destas duas quantidades, por sua vez, resulta na densidade de energia eletrônica da distribuição de carga (BADER, 1990),

$$
H_{C P}=G_{C P}+V_{C P}
$$

A Teoria Quântica de Átomos em Moléculas propicia, além da descrição topológica do sistema, a obtenção de propriedades moleculares a partir da integração da densidade eletrônica associada ao volume atribuído ao átomo (RODRIGUES et al., 2008; Da SILVA, 2009; BADER et al., 1990). O volume atômico é o espaço definido por superfícies de fluxo zero, que delimitam o átomo no interior da molécula, e, eventualmente, por superfícies de isodensidade que demarcam a região exterior da molécula. Como a densidade eletrônica da molécula estende-se, a princípio, ao infinito, a superfície de isodensidade com $\rho(\vec{r})=$ 0,001 u.a. é usualmente escolhida como limite externo da mesma, porque contempla o volume experimental de van der Waals na fase gasosa e, geralmente, envolve $99 \%$ da população eletrônica da molécula (BADER, 1990). A Figura 2 mostra o formato dos átomos do hidreto de lítio ( $\mathrm{LiH})$ segundo estes limites.

Figura 2 - Forma dos átomos da molécula hidreto de lítio $(\mathrm{LiH})$ segundo as delimitações das superfícies de fluxo zero dadas pela partição QTAIM

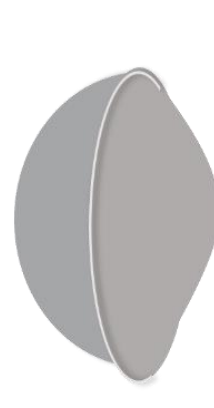

$\mathbf{L i}$

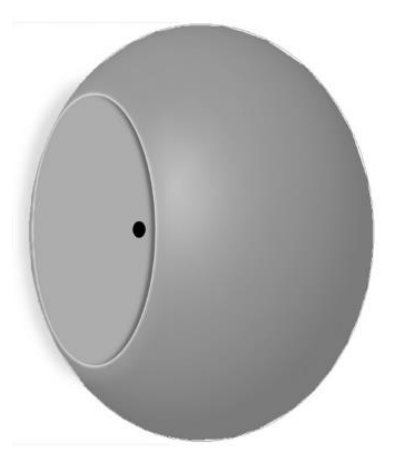

$\mathbf{H}$

Fonte: Adaptado do site: https://www.chemistry.mcmaster.ca/aim/aim_3.html. Acesso em 07 de set de 2019.

A população total de elétrons de um átomo $\Omega(N(\Omega))$ em uma molécula, por exemplo, é obtida por (MATTA e BOYD, 2007): 


$$
N(\Omega)=\int_{\Omega} \rho(\vec{r}) d \vec{r}
$$

Desta forma, a carga atômica, $q(\Omega)$, é obtida pela subtração de $N(\Omega)$ da carga nuclear $Z_{\Omega}$, ou seja,

$$
q(\Omega)=Z_{\Omega}-N(\Omega)
$$

Isoladamente, as cargas QTAIM não reproduzem de forma adequada o momento dipolar molecular e o potencial eletrostático ao redor de uma molécula, pois não levam em consideração a deformação da nuvem eletrônica do átomo na molécula (polarização atômica). Então, esta assimetria na distribuição de carga eletrônica em torno dos núcleos dos átomos origina um momento de dipolo atômico (BADER, 1990). Assim, de acordo com o formalismo QTAIM e para obter o resultado líquido do momento de dipolo molecular, é necessário efetuar uma soma vetorial da contribuição de carga e de dipolo atômico,

$$
\vec{\mu}=\sum_{\Omega} \vec{\mu}_{P A}(\Omega)+\sum_{\Omega} q(\Omega) \vec{R}_{\Omega}
$$

em que $q(\Omega)$ e $\vec{\mu}_{P A}(\Omega)$ são a carga e o dipolo atômico do átomo $\Omega$ com coordenadas $\vec{R}_{\Omega}$ (vetor de componentes $X_{\Omega}, Y_{\Omega}$ e $Z_{\Omega}$ ) em termos de uma origem arbitrária. A transformação do segundo termo da equação anterior resulta em (MATTA e BOYD, 2007):

$$
\vec{\mu}=\sum_{\Omega} \vec{\mu}(\Omega)=\sum_{\Omega}\left\{\vec{\mu}_{P A}+\sum_{\Omega^{\prime} \neq \Omega} q\left(\Omega^{\prime}\right)\left[\vec{R}_{B C P}\left(\Omega \mid \Omega^{\prime}\right)-\vec{R}_{\Omega}\right]\right\}
$$

na qual:

$$
\begin{aligned}
& \vec{\mu}(\Omega)=\vec{\mu}_{P A}(\Omega)+\vec{\mu}_{C T}(\Omega), \\
& \vec{\mu}_{P A}(\Omega)=\int_{\Omega} \vec{r}_{\Omega} \rho(\vec{r}) d \vec{r} \\
& \vec{\mu}_{C T}(\Omega)=\sum_{\Omega^{\prime} \neq \Omega} q\left(\Omega^{\prime}\right)\left[\vec{R}_{B C P}\left(\Omega \mid \Omega^{\prime}\right)-\vec{R}_{\Omega}\right]
\end{aligned}
$$


em que $\vec{R}_{B C P}\left(\Omega \mid \Omega^{\prime}\right)$ é a posição do ponto crítico de ligação (BCP) entre átomos vizinhos $\Omega$ e $\Omega^{\prime}, \rho(\vec{r})$ é a densidade eletrônica e $\vec{r}_{\Omega}=\vec{r}-\vec{R}_{\Omega}$ é a vetor posição com a origem transladada para o núcleo do átomo.

\section{2 Átomos Quânticos Interagentes (IQA)}

Recentemente, utilizando os fundamentos da QTAIM, Pendás e colaboradores (PENDÁS, BLANCO e FRANCISCO, 2005; BLANCO, PENDÁS e FRANCISCO, 2006) introduziram uma maneira de particionar a energia eletrônica de uma molécula em contribuições intra- e interatômicas e, assim, abordando conceitos químicos em termos energéticos. Neste caso,

$$
E=\sum_{\Omega} E_{\text {Intra }}(\Omega)+\sum_{\Omega} \sum_{\Omega^{\prime}>\Omega} E_{\text {Inter }}\left(\Omega, \Omega^{\prime}\right)
$$

A energia intra-atômica compreende a energia cinética eletrônica do átomo $\Omega, T(\Omega)$, a energia de atração entre a densidade eletrônica de $\Omega$ e seu núcleo, $V_{e n}(\Omega)$, e a energia de interação elétron-elétron do átomo $\Omega, V_{e e}(\Omega)$, ou seja,

$$
E_{\text {Intra }}(\Omega)=T(\Omega)+V_{e n}(\Omega)+V_{e e}(\Omega)
$$

De modo similar, a energia interatômica pode ser subdividida em energia de atração entre o núcleo do átomo $\Omega$ e a distribuição de densidade eletrônica do átomo $\Omega^{\prime}, V_{n e}\left(\Omega, \Omega^{\prime}\right)$, e vice-versa, $V_{e n}\left(\Omega, \Omega^{\prime}\right)$, energia de repulsão internuclear, $V_{n n}\left(\Omega, \Omega^{\prime}\right)$ e interação elétron-elétron $V_{e e}\left(\Omega, \Omega^{\prime}\right)$. Este último termo pode ser particionado ainda em energia Coulômbica, $V_{e e}^{C}\left(\Omega, \Omega^{\prime}\right)$, e energia de troca-correlação, $V_{X C}\left(\Omega, \Omega^{\prime}\right.$ ) (PENDÁS, BLANCO e FRANCISCO, 2005; BLANCO, PENDÁS e FRANCISCO, 2006),

$$
E_{\text {Inter }}\left(\Omega, \Omega^{\prime}\right)=V_{n n}\left(\Omega, \Omega^{\prime}\right)+V_{n e}\left(\Omega, \Omega^{\prime}\right)+V_{e n}\left(\Omega, \Omega^{\prime}\right)+V_{e e}^{C}\left(\Omega, \Omega^{\prime}\right)+V_{X C}\left(\Omega, \Omega^{\prime}\right) .
$$

Então, a energia de interação total pode ser dividida em um termo eletrostático clássico, $V_{c l}\left(\Omega, \Omega^{\prime}\right)=V_{n n}\left(\Omega, \Omega^{\prime}\right)+V_{n e}\left(\Omega, \Omega^{\prime}\right)+V_{e n}\left(\Omega, \Omega^{\prime}\right)+V_{e e}^{C}\left(\Omega, \Omega^{\prime}\right)$, e o termo de trocacorrelação ou termo não-clássico, $V_{X C}\left(\Omega, \Omega^{\prime}\right)=V_{X}\left(\Omega, \Omega^{\prime}\right)+V_{C O R R}\left(\Omega, \Omega^{\prime}\right)$. Assim, de acordo 
com Pendás, Blanco e Francisco (2005, 2006), a energia de interação IQA pode ser representada por:

$$
E_{\text {Inter }}\left(\Omega, \Omega^{\prime}\right)=V_{c l}\left(\Omega, \Omega^{\prime}\right)+V_{X C}\left(\Omega, \Omega^{\prime}\right)
$$

\section{Modelo de partição Carga - Fluxo de Carga - Fluxo de Dipolo em termos dos multipolos da Teoria Quântica de Átomos em Moléculas (CCFDF/QTAIM)}

Uma ferramenta valiosa para analisar as derivadas do momento de dipolo molecular, que, por sua vez, estão correlacionadas com as intensidades de infravermelho, é o modelo de Carga - Fluxo de Carga - Fluxo de Dipolo (CCFDF) em termos dos multipolos da Teoria Quântica de Átomos em Moléculas (QTAIM) (HAIDUKE e BRUNS, 2005; BADER, 1990). De acordo com este modelo, as derivadas dos componentes do momento do dipolo molecular $\left(\mu_{r}\right.$, sendo $r=x, y$ e $z$ ) durante vibrações associadas a cada modo normal $\left(Q_{i}\right)$ podem ser escritas em termos de quantidades que descrevem o deslocamento de cargas atômicas (carga, C), transferência de carga eletrônica entre átomos (fluxo de carga, CF) e mudanças na polarização de átomos (fluxo de dipolo, DF), isto é,

$$
\frac{\partial \mu_{r}}{\partial Q_{i}}=\sum_{\Omega} q^{0}(\Omega) \frac{\partial r_{\Omega}^{0}}{\partial Q_{i}}+\sum_{\Omega} r_{\Omega} \frac{\partial q_{\Omega}}{\partial Q_{i}}+\sum_{\Omega} \frac{\partial \mu_{r}(\Omega)}{\partial Q_{i}}=\left(\frac{\partial \mu_{r}}{\partial Q_{i}}\right)_{C}+\left(\frac{\partial \mu_{r}}{\partial Q_{i}}\right)_{C F}+\left(\frac{\partial \mu_{r}}{\partial Q_{i}}\right)_{D F},
$$

na qual $q(\Omega)$ e $\mu_{r}(\Omega)$ representam a carga e o momento de dipolo atômico do átomo $\Omega$ (HAIDUKE e BRUNS, 2005).

Portanto, na partição CCFDF/QTAIM, as intensidades de infravermelho $(A)$ também são interpretadas por meio das contribuições de carga, fluxo de carga e fluxo de dipolo, juntamente com os termos cruzados entre pares dessas quantidades (De OLIVEIRA, 1999; PERSON e NEWTON, 1974; SILVA et al., 2012), ou seja,

$$
A_{i}=\frac{N_{A} \pi}{3 c^{2}}\left[\left|\left(\frac{\partial \vec{\mu}}{\partial Q_{i}}\right)_{C}\right|^{2}+\left|\left(\frac{\partial \vec{\mu}}{\partial Q_{i}}\right)_{C F}\right|^{2}+\left|\left(\frac{\partial \vec{\mu}}{\partial Q_{i}}\right)_{D F}\right|^{2}+2\left(\frac{\partial \vec{\mu}}{\partial Q_{i}}\right)_{C} \cdot\left(\frac{\partial \vec{\mu}}{\partial Q_{i}}\right)_{C F}+2\left(\frac{\partial \vec{\mu}}{\partial Q_{i}}\right)_{C} \cdot\left(\frac{\partial \vec{\mu}}{\partial Q_{i}}\right)_{D F}+2\left(\frac{\partial \vec{\mu}}{\partial Q_{i}}\right)_{C F} \cdot\left(\frac{\partial \vec{\mu}}{\partial Q_{i}}\right)_{D F}\right]
$$

ou, sucintamente, 


$$
A=A^{C}+A^{C F}+A^{D F}+A^{C \times C F}+A^{C \times D F}+A^{C F \times D F}
$$

na qual $N_{A}$ e $c$ representam o número de Avogadro e a velocidade da luz, respectivamente.

Os primeiros três termos descrevem as contribuições de carga, fluxo de carga e fluxo de dipolo correspondentes a uma intensidade vibracional fundamental. Os três últimos termos são os termos cruzados entre as quantidades mencionadas e podem ser positivos, o que resulta em um aumento da intensidade total, ou negativos, acarretando uma redução do valor desta propriedade. Esta partição em contribuições clássicas permite compreender de modo mais fundamental as mudanças das intensidades de infravermelho durante diversos fenômenos de interesse químico.

\section{Teoria do Funcional de Densidade (DFT)}

O tratamento teórico empregado nas aplicações da Teoria Quântica de Átomos em Moléculas para os estudos dos fenômenos de dimerização de ácidos carboxílicos, complexação de carbonilas metálicas, excitação eletrônica de moléculas diatômicas simples e reatividade e regiosseletividade de compostos aromáticos substituídos em reações de substituição eletrofílica (capítulos 2, 3 e 4) será realizado por meio da Teoria do Funcional de Densidade. A Teoria do funcional da densidade (DFT) fundamenta-se em dois teoremas propostos por Hohenberg e Kohn (1964) e no desenvolvimento de um conjunto de equações formuladas por Kohn e Sham na metade de 1960. O primeiro teorema afirma que "a energia do estado fundamental a partir da equação de Schrödinger pode ser expressa como um funcional único da densidade eletrônica (SHOLL e STECKEL, 2009).” Então, podemos expressar esta energia da seguinte forma:

$$
E=[\rho(\vec{r})]
$$

em que $\rho(\vec{r})$ é a densidade eletrônica. Além disto, a densidade eletrônica determina todas as propriedades do sistema (LEVINE, 2000; MORGON e COUTINHO, 2007).

Embora o primeiro teorema de Hohenberg-Kohn afirme que a energia, assim como as demais propriedades do sistema, pode ser obtida por meio de um funcional da densidade eletrônica, o teorema não aborda sobre qual seria o formato matemático deste funcional. $\mathrm{O}$ segundo teorema de Hohenberg-Kohn (1964) define uma importante propriedade do 
funcional: "a densidade eletrônica que minimiza a energia do funcional exato é a verdadeira densidade eletrônica correspondente à solução completa da equação de Schrödinger." Se a forma do funcional exato fosse conhecida, então seria possível variar a densidade eletrônica até que a energia do funcional fosse minimizada, fornecendo, assim, um procedimento operacional para encontrar a densidade eletrônica (SHOLL e STECKEL, 2009). Este princípio variacional é empregado na prática com formas aproximadas de funcionais.

Segundo as equações Kohn-Sham, a energia do funcional, $E[\rho]$ pode ser escrita como:

$$
E[\rho]=T[\rho]+J[\rho]+E_{n e}[\rho]+E_{X C}[\rho]
$$

na qual $T[\rho]$ é a energia cinética dos elétrons, $J[\rho]$, a energia de repulsão clássica elétronelétron, $E_{n e}[\rho]$, a energia de atração elétron-núcleo e $E_{X C}[\rho]$, a energia de troca-correlação, que inclui todos os efeitos quanto-mecânicos que não foram considerados nos termos expressos analiticamente, como troca e correlação de Coulomb, correção para auto-interação e diferença entre as energias cinéticas do sistema real e do não-interagente (SHOLL e STECKEL, 2009).

Para resolver as equações de Kohn-Sham é necessário conhecer a densidade eletrônica. Mas, para encontrá-la, deve-se determinar as funções de onda de um elétron e, para conhecer estas funções, as equações de Kohn-Sham precisam ser resolvidas. A fim de romper este círculo, o problema é tratado de forma interativa segundo o procedimento a seguir (SHOLL e STECKEL, 2009):

1) Defina uma densidade eletrônica inicial como tentativa $(\rho(\vec{r}))$;

2) Resolva as equações de Kohn-Sham definidas usando a densidade eletrônica tentativa para encontrar as funções de onda de um elétron;

3) Calcule a densidade eletrônica definida pelas funções de onda de Kohn-Sham de um elétron a partir do passo $2, \rho_{K S}(\vec{r})=2 \sum_{i} \psi_{i}^{*}(\vec{r}) \psi_{i}(\vec{r})$;

4) Compare a densidade eletrônica calculada, $\rho_{K S}(\vec{r})$, com a densidade eletrônica utilizada para resolver as equações de Kohn-Sham, $\rho(\vec{r})$. Se as duas densidades são iguais, então, esta é densidade eletrônica do estado fundamental e pode ser empregada para o cálculo da energia total. Se as duas densidades forem diferentes, a densidade eletrônica tentativa deve ser modificada de alguma forma. Quando isso for feito, o processo começa novamente a partir do passo 2 . 
Esta forma interativa de resolver o problema permite uma solução auto consistente das equações de Kohn-Sham. Para solucionar estas equações, é necessário especificar o formato do funcional de troca-correlação (cuja existência é garantida pelo teorema de HohenbergKohn), embora a sua forma exata não seja conhecida para quase a totalidade dos sistemas. Há apenas alguns casos no qual este funcional pode ser derivado exatamente, por exemplo, o gás uniforme de elétrons e sistemas monoeletrônicos. Nesta situação, a densidade de elétrons é constante em todos os pontos do espaço e o potencial de troca-correlação pode ser calculado por meio da seguinte equação (SHOLL e STECKEL, 2009):

$$
V_{X C}(\vec{r})=V_{X C}^{G a ́ s ~ d e ~ e l e ́ t r o n ~}[\rho(\vec{r})]
$$

Esta aproximação utiliza apenas a densidade local para definir o funcional de trocacorrelação, sendo denominada de aproximação da densidade local (LDA). Além da LDA, a próxima classe de funcionais utiliza informações sobre a gradiente da densidade eletrônica. Esta abordagem define uma aproximação de gradiente generalizado (GGA) em que a densidade eletrônica não é uniforme (LEVINE, 2000):

$$
E_{X C}^{G G A}[\rho]=f[\rho(\vec{r}), \nabla \rho(\vec{r})] d \vec{r}
$$

Os funcionais Perdew-Wang 91 (PW91) e o Perdew-Burke-Ernzerhof (PBE) são baseados nesta aproximação. Em geral, a função $f[\rho(\vec{r}), \nabla \rho(\vec{r})]$ é desenvolvida empregandose parâmetros que são ajustáveis a partir de relações exatas ou de valores de propriedades. Outra classe de funcionais é constituída pelos meta-GGA, que incluem informações como a densidade eletrônica, seu gradiente e seu Laplaciano, ou seja, $\rho(\vec{r}), \nabla \rho(\vec{r})$ e $\nabla^{2}(\vec{r})$, respectivamente.

Funcionais baseados nesta quantidade não podem ser avaliados em uma localização espacial particular a menos que a densidade eletrônica seja conhecida em todos os pontos do espaço. A inclusão de contribuições de energia de troca exata em um funcional GGA ou metaGGA gera outra classe de funcionais, os funcionais híbridos. De modo geral, os funcionais híbridos incorporam o termo de troca exato (escritos a partir de orbitais de Kohn-Sham (1965)), análogo ao presente na teoria Hartree-Fock, com funcionais de troca e correlação 
eletrônica provenientes da Aproximação da Densidade Local (LDA) e da Aproximação do Gradiente Generalizado (GGA) (LEVINE, 2000):

Atualmente, o funcional híbrido mais popular é conhecido como B3LYP e foi sugerido por Koch et al., em 1994. A expressão para a energia de troca-correlação para este funcional é:

$$
E_{X C}^{B 3 L Y P}=(1-a-b) E_{X}^{L D A}+a E_{X}^{H F}+b E_{X}^{B 88}+c E_{C}^{L Y P}+(1-c) E_{C}^{L D A}
$$

em que $a=0,20, b=0,72$ e $c=0,81 . E_{X}^{L D A}$ e $E_{c}^{L D A}$ são, respectivamente, o funcional de troca da expressão de Vosko, Wilk e Nusair (1980) e o termo de correlação, ambos da Aproximação da Densidade Local, e $E_{X}^{B 88}$ e $E_{c}^{L Y P}$ são, respectivamente, a correção do funcional de troca de Becke (1988) e o funcional de correlação de Lee, Yang e Parr (1988), oriundos da GGA.

O funcional PBE0 também faz parte da família dos híbridos e tem sido bastante aplicado em estudos de estados excitados de moléculas orgânicas. Ele inclui uma fração de $25 \%$ do termo de troca exata do Hartree-Fock $\left(E_{X}^{H F}\right)$ e o funcional GGA de troca-correlação, PBE (ADAMO e BARONE, 1999):

$$
E_{X C}^{P B E 0}=E_{X C}^{P B E}+0,25\left(E_{X}^{H F}-E_{X}^{P B E}\right)
$$

No decorrer deste trabalho, utilizaremos ainda o funcional B2PLYP, proposto por GRIMME (2006) e pertencente à família dos duplos híbridos, que tem sido bem-sucedido na predição de diversas propriedades físico-químicas. O B2PLYP é baseado em uma mistura de aproximações de gradiente generalizado para troca de Becke (1993) (B) e para correlação de Lee, Yang e Parr (LYP) (1988), incluindo uma parcela de troca exata Hartree-Fock de 53\% e outra de correlação de segunda ordem perturbativa (PT2), obtidas a partir dos orbitais de Kohn-Sham e de seus autovalores. Então,

$$
E_{X C}^{B 2 P L Y P}=(1-0,53) B_{X}^{88}+0,53 E_{X}^{H F}+(1-0,27) E_{C}^{L Y P}+0,27 E_{C}^{P T 2}
$$




\section{Teoria do Funcional de Densidade Dependente do Tempo (TD-DFT)}

O processo de interação de sistemas moleculares com a radiação eletromagnética é descrito na mecânica quântica por meio da seguinte equação em termos das coordenadas das partículas $(q)$ e do tempo $(t)$ :

$$
\widehat{H}(q, t) \psi(q, t)=i \frac{\partial \Psi(q, t)}{\partial t}
$$

Neste caso, o operador Hamiltoniano é escrito em função da energia cinética dos elétrons, da repulsão intereletrônica, da radiação eletromagnética interagindo com o sistema molecular e da atração elétron-núcleo, respectivamente (YANG, 1988):

$$
\widehat{H}(q, t)=-\frac{1}{2} \sum_{i=1}^{N} \nabla_{i}^{2}+\sum_{i=1}^{N} \sum_{j>1}^{N} \frac{1}{r_{i j}}+E f(t) \operatorname{sen}(\omega t) \vec{r}_{i} \cdot \alpha-\sum_{j=1}^{M} \frac{Z_{j}}{\left|\vec{r}_{i}-\vec{R}_{i}\right|}
$$

em que $\alpha, \omega e E$ são a polarização, a frequência e a amplitude da radiação, enquanto $f(t)$ representa a sua evolução temporal.

A solução da equação 29 é bastante complexa, pois seria necessário obter uma função de onda do estado fundamental e de todos os estados excitados. No entanto, o Teorema de Runge e Gross (1984) estabelece que todas as propriedades observáveis podem ser calculadas mediante a densidade eletrônica. Um modo de se aplicar a TD-DFT seria por meio de equações escritas com o auxílio do sistema de elétrons não-interagentes, nas quais a densidade pode ser obtida por meio dos orbitais de Kohn-Sham dependentes do tempo (VAN LEEUWEN, 1999):

$$
\rho(\vec{r}, t)=\sum_{i=1}^{N}\left|\varphi_{i}(\vec{r}, N)\right|^{2}
$$

e o potencial $v_{S}(\vec{r}, t)$, pode ser definido como:

$$
v_{s}(\vec{r}, t)=v_{e x t}(\vec{r}, t)+\int \frac{\rho\left(\vec{r}^{\prime}, t\right)}{\left|\vec{r}_{i}-\vec{r}_{i}^{\prime}\right|} d \vec{r}_{i}+v_{x c}(\vec{r}, t)
$$

Assim, para se encontrar os orbitais: 


$$
i \frac{\partial}{\partial t}\left(\varphi_{i}(\vec{r}, t)\right)=\left[-\frac{1}{2} \nabla^{2}+v_{s}(\vec{r}, t)\right] \varphi_{i}(\vec{r}, t)
$$

O potencial de troca-correlação, $v_{x c}(\vec{r}, t)$, é uma quantidade difícil de se obter porque sua representação matemática deveria considerar a "história do sistema" até um determinado instante de tempo $t$. Na prática, porém, utiliza-se a aproximação adiabática, visto que o principal interesse é determinar as energias de transições eletrônicas e as intensidades de bandas vibracionais (MARQUES et. al., 2006; MARQUES e GROSS, 2004; YANG, 1988):

$$
v_{x c}(\vec{r}, t) \approx \frac{\delta E_{X C}[\rho(\vec{r}, t)]}{\delta[\rho(\vec{r}, t)]}
$$

De modo geral, os programas computacionais utilizam uma abordagem simplificada, a teoria de resposta linear, na qual a perturbação, representada por um potencial dependente do tempo, é aplicada a um sistema no estado fundamental e, em seguida, a densidade eletrônica é escrita como uma série de Taylor (MARQUES et. al., 2006; MARQUES e GROSS, 2004):

$$
\rho(\vec{r}, t)=\rho_{0}(\vec{r}, t)+\rho_{1}(\vec{r}, t)+\rho_{2}(\vec{r}, t)+\cdots
$$

No caso da resposta linear, inclui-se apenas a correção de primeira ordem:

$$
\rho(\vec{r}, t)=\rho_{0}(\vec{r}, t)+\rho_{1}(\vec{r}, t)
$$

Assim,

$$
\rho_{1}(\vec{r}, t)=\int_{0}^{\infty} \int \chi\left(\vec{r} t, r^{\prime} t\right) \delta v_{\text {ext }}\left(\vec{r}^{\prime}, t^{\prime}\right) d \vec{r}^{\prime}
$$

no qual $\chi\left(\vec{r} t, r^{\prime} t\right)$ é a função de resposta interagente (YANG, 1988):

$$
\chi\left(\vec{r} t, r^{\prime} t\right)=\left.\frac{\delta \rho(\vec{r}, t)}{\delta v_{\text {ext }}\left(\vec{r}^{\prime}, t^{\prime}\right)}\right|_{v_{\text {ext }, 0}}
$$

Esta equação pode ser escrita a partir das soluções da teoria do funcional de densidade do estado fundamental: 


$$
\chi_{K S}\left(\vec{r} t, r^{\prime} t\right)=\left.\frac{\delta \rho(\vec{r}, t)}{\delta v_{K S}\left(\vec{r}^{\prime}, t^{\prime}\right)}\right|_{v_{K S, 0}}
$$

Assim, para fazer um cálculo TD-DFT (YANG, 1988), primeiramente, escolhe-se um funcional e um conjunto de funções de base, resolve-se as equações de Kohn-Sham do estado fundamental e, por fim, é necessário encontrar os pólos da seguinte equação no domínio das frequências:

$$
\begin{aligned}
& \chi\left(\vec{r} t, r^{\prime} t\right)=\chi_{K S}\left(\vec{r} t, r^{\prime} t\right)+\iiint \int \chi_{K S}\left(\vec{r} t, r^{\prime} t\right)\left[\frac{\delta\left(t_{1}-t_{2}\right.}{\left|\vec{r}_{1}-\vec{r}_{2}\right|}+f_{X C}\left(\vec{r}_{1} t_{1}, \vec{r}_{2} t_{2}\right)\right] . \\
& \chi\left(\vec{r} t, r^{\prime} t\right) d t_{1} d t_{2} d \vec{r}_{1} d \vec{r}_{2}
\end{aligned}
$$

na qual,

$$
f_{X C}\left(\vec{r}_{1} t_{1}, \vec{r}_{2} t_{2}\right)=\frac{\delta v_{X C}\left(\vec{r}_{1}, t_{1}\right)}{\delta \rho\left(\vec{r}_{2}, t_{2}\right)}
$$

A aplicação de uma transformada Fourier permite realizar esta mudança do domínio do tempo para o das frequências, sendo que os pólos da equação 40, são pontos de singularidade da função resposta, que fornecem as frequências de transição eletrônicas. Além disso, as intensidades das bandas podem ser obtidas por meio da força do oscilador, visto que estas quantidades são proporcionais.

\section{REFERÊNCIAS}

ADAMO, C. e BARONE V. Toward reliable density functional methods without adjustable parameters. The Journal of Chemical Physics, v. 110, p. 6158-6159, 1999.

BADER, R. F. W. Atoms in Molecules: A Quantum Theory. $1^{\text {a }}$ ed. Oxford, UK: Clarendon Press, 1990.

BECKE, A. D. Density-functional exchange-energy approximation with correct asymptotic behavior. Physical Review A, v. 38, p. 3098-3100, 1988.

BECKE, A. D. Density-functional thermochemistry III. The role of exact exchange. The Journal of Chemical Physics, v. 98, p. 5648-5652, 1993. 
BLANCO, M. A.; PENDÁS, A. M. e FRANCISCO, E. Interacting quantum atoms: a correlated energy decomposition scheme based on the quantum theory of atoms in molecules. Journal of Chemical Theory and Computation, v. 1, p. 1096-1109, 2005.

Da SILVA, J. V.; OLIVEIRA, A. E.; HASE e Y. BRUNS, R. E. Quantum Theory Atoms in Molecules Charge - Charge Flux - Dipole Flux Models for the Infrared Intensities of Benzene and Hexafluorobenzene. Journal Physical Chemistry A, v. 113, p. 7972-7978, 2009.

De OLIVEIRA, A. E. e GUADAGNINE, P. H. A. Simple Potential Model Criterion for the Quality of Atomic Charges. Journal Physical Chemistry A, v. 103, p. 4918-4924, 1999.

GARCÍA-REVILLA, M. A.; CORTÉS-GÚZMAN, F.; ROCHA-RINZA, T. e HERNÁNDEZ-TRUJILLO, J. Latin American contributions to quantum chemical topology. International of Quantum Chemistry, v. 119, p. 1-26, 2019.

GRIMME, S. Semiempirical hybrid density functional with perturbative second-order correlation. The Journal of Chemical Physics, v. 124, p. 1-16, 2006.

GRABOWSKI, S. J. What is the covalency of hydrogen bond? Chemical Reviews, v. 111, p. 2597-2625, 2011.

HAIDUKE, R. L. A. e BRUNS, R. E. An atomic charge-charge flux-dipole flux atom-inmolecule decomposition for molecular dipole-moment derivatives and infrared fundamental intensities. Journal of Chemical Physics A, v. 109, p. 2680-2688, 2005.

HOHENBERG, O. e KOHN, W. Inhomogeneous electron gas. Physical Review, v. 136, p. 864-871, 1964.

KOHN, W.; SHAM, L. J. Self-consistent equations including exchange and correlation effects. Physical Review, v. 140, n. 4A, p. 1133-1138, 1965.

KOCH, W. e HOLTHAUSEN, M. C. A Chemist' guide to Density Functional Theory. $2^{\mathrm{a}}$ ed. Weinheim. Germany: Wiley-VCH VelagGmbH, 2000

LEE, C.; YANG, W. e PARR, R. G. Development of the Colle-Salvetti correlation-energy formula into a functional of the electron density. Physical Review, v. 37, p. 785-789, 1988.

LEVINE, I. N. Quantum Chemistry. Upper Sanddle River: Prentice Hall, 2000. 585-591 p.

MARQUES, M. A. L. e GROSS, E. K. U. Time-dependent density functional theory. Annual Review Physical Chemistry, v. 55, p. 427-455, 2004.

MARQUES, M. A. L., ULLRICH, C. A.; NOGUEIRA, F.; RUBIO, A.; BURQUE, K. E. e GROSS, E. K. U. Time-dependent density functional theory. Berlin Heidelberg: Springer, 2006, $591 \mathrm{p}$.

MATTA, C. F. e BOYD, R. J. The Quantum Theory of Atoms in Molecules From Solid State to DNA and Drug Design, Weinheim: Wiley-Vch, 2007.

MORGON, N. H. e COUTINHO, K. Métodos de Química Teórica e Modelagem

Molecular. São Paulo: Livraria da Física, 2007. 134 p. 
PENDÁS, A. M.; BLANCO, M. A. e FRANCISCO, E. The nature of the hydrogen bond: a synthesis from the interacting quantum atoms picture. The Journal of Chemical Physics, v. $125,184112,2006$.

PERSON, W. B. e NEWTON, J. H. Dipole moment derivatives and infrared intensities. I Polar tensors. The Journal of Chemical Physics, v. 61, p. 1040-1049, 1974.

RODRIGUES, E. F. F.; DE SÁ, E. L. e HAIDUKE, R. L. A. Electrostatic properties of small molecules by means of atomic multipoles from the Quantum Theory of Atoms in Molecules. International Journal of Quantum Chemistry, v. 108, p. 2417-2427, 2008.

RUNGE, E. e GROSS, E. K. U., Density-functional theory for time-dependent systems. Physical Review Letter, v. 52, p. 997-1000, 1974.

SHOLL, S. S. e STECKEL, J. A. Density Functional Theory: A Practical Introduction. New Jersey: Wiley, 2009. 1-10 p.

SILVA, A. F.; RICHTER, W. E.; MENESES, H. G. C.; FARIA, S. H. D. M. e BRUNS, R. E. How accessible is atomic charge information from intensities? A QTAIM/CCFDF interpretation. The Journal of Physical Chemistry A, v. 116, p. 8238-8249, 2012.

STEPHENS, J.; DEVLIN, F. J.; CHABALOWSKI, C. F. e FRISCH, M. J. Ab initio calculation of vibrational absorption and circular dichroism spectra using density functional force fields. The Journal of Physical Chemistry, v. 98, p. 11623-11627, 1994.

VAN LEEUWEN, R. Mapping from densities to potentials in time-dependent densityfunctional theory. Physical Review Letter, v. 82, p. 3863-3866, 1999.

VOSKO, S. H.; WILK, L. e NUSAIR, M. Accurate spin-dependent electron liquid correlation energies for local spin density calculations: a critical analysis. Canadian Journal of Physics, v. 58, p. 1200-1211, 1980.

YANG, W. Dynamic linear response of many-electron systems: An integral formulation of density-functional theory. Physical Review A, v. 38, p. 5512-5519, 1988. 
Capítulo 2

Análise das intensidades de infravermelho dos modos de estiramento da hidroxila de dímeros de ácidos carboxílicos por meio do modelo de Carga -

Fluxo de carga - Fluxo de Dipolo 


\section{INTRODUÇÃO}

A ligação de hidrogênio (HB - do inglês "hydrogen bond") possui grande relevância em processos bioquímicos e suas características funcionais são tão essenciais que a vida não seria possível sem este tipo de interação, pois é ela que determina a estrutura organizacional e os modos de reconhecimento e associação entre biomoléculas nos seres vivos, como proteínas, polissacarídeos e ácidos nucleicos (JEFFREY e SAENGER, 1991; DESIRAJU e STEINER, 1999). A IUPAC (2011) define a ligação de hidrogênio como uma "interação atrativa entre um átomo de hidrogênio de uma molécula ou de um fragmento molecular $X-H$, em que X é mais eletronegativo que $H$, e um átomo ou grupo de átomos de uma mesma ou de diferentes moléculas" (ARUNAN et al., 2011). Levando-se em conta esta definição, X-H...Y representa a ligação de hidrogênio composta por um monômero doador de próton genérico, $\mathrm{X}-\mathrm{H}$, e uma unidade aceptora de próton com extremidade simbolizada por Y (Da SILVA e HAIDUKE, 2019).

As ligações de hidrogênio podem ser classificadas com base na sua energia de ligação como fracas $\left(1-4 \mathrm{kcal} \mathrm{mol}^{-1}\right)$, médias $\left(4-15 \mathrm{kcal} \mathrm{mol}^{-1}\right)$ e fortes $\left(15-40 \mathrm{kcal} \mathrm{mol}^{-1}\right)$. Esta energia é descrita pela diferença entre a energia total do complexo e as energias dos monômeros isolados (MATTA e BOYD, 2007). Rozas et al. (2000) propôs outra definição baseada não apenas na energia, mas também em dados topológicos de densidade eletrônica. Para ligações de hidrogênio fracas, a energia da HB deve ser menor que $12 \mathrm{kcal} \mathrm{mol}^{-1}$, enquanto o Laplaciano da densidade eletrônica $\left(\nabla^{2} \rho_{\mathrm{H}} \ldots \mathrm{Y}\right)$ e sua densidade de energia $\left(H_{b}\right)$ no BCP da ligação de hidrogênio (H...Y) devem ser positivos. Para ligações de hidrogênio de força média, a energia envolvida deve estar entre 12 e $24 \mathrm{kcal} \mathrm{mol}^{-1}$, ao mesmo tempo em que $\nabla^{2} \rho_{\mathrm{H} \ldots \mathrm{Y}}>0$ e $H_{b}<0$. Por sua vez, a energia de HBs fortes é maior do que $24 \mathrm{kcal} \mathrm{mol}^{-1} \mathrm{e}$ ambos os parâmetros topológicos, $\nabla^{2} \rho_{\mathrm{H} . . . \mathrm{Y}}$ e $H_{b}$, apresentam valores negativos.

O fato de o Laplaciano da densidade eletrônica ser negativo é um indicativo de ligações covalentes e também é uma característica de ligações de hidrogênio fortes (GRABOWSKI, 2007). Portanto, as ligações de hidrogênio podem apresentar certo caráter covalente (GRABOWSKI, 2007; ISAACS, SHUKLA e PLATZMAN, 1999). Contudo, o sinal do Laplaciano isoladamente não é suficiente para classificar uma interação porque valores positivos desta propriedade já foram encontrados para ligações CO duplas e triplas (CREMER e KRAKA, 1984). O sinal negativo da densidade de energia eletrônica total no BCP é requerido, portanto, para classificar uma ligação como covalente (GRABOWSKI, 2007 e 2011). 
A densidade de energia eletrônica total $\left(H_{b}\right)$ determina se a energia cinética ou potencial domina no BCP e se a densidade eletrônica neste ponto possui efeito estabilizante ou desestabilizante sobre o sistema (GRABOVSKI, 2011). Então, uma interação apresenta caráter covalente se a energia potencial domina sobre a energia cinética $\left(H_{b}<0\right)$ de modo que a densidade eletrônica estabiliza as espécies. Contudo, as ligações de hidrogênio diferem das ligações covalentes tradicionais por serem facilmente deformadas e, além disso, exibirem propriedades de grupo (JEFFREY e SAENGER, 1991). Quantidades QTAIM, tais como a densidade de elétrons, seu Laplaciano $\left(\nabla^{2} \rho\right)$ e a densidade de energia nos pontos críticos de ligação (BCPs), como discutido anteriormente, têm sido utilizadas para caracterizar ligações de hidrogênio inter- e intramoleculares (GRABOWSKI, 2007; BADER, 1990; KOCH e POPELIER, 1995) e, além disso, a partição energética de Átomos Quânticos Interagentes (IQA) (PENDÁS, BLANCO e FRANCISCO, 2005 e 2006) deve permitir avaliar a estabilização e a desestabilização das ligações de hidrogênio e das ligações envolvidas diretamente neste processo.

A formação da ligação de hidrogênio modifica a estrutura eletrônica dos monômeros envolvidos, por exemplo, em ligações de hidrogênio fortes, nas quais as ligações X-H podem ser alongadas de tal maneira que esta perturbação pode ser propagada para outras ligações adjacentes (JEFFREY e SAENGER, 1991). Estas mudanças nas ligações covalentes que participam diretamente da HB são mais facilmente detectadas por métodos espectroscópicos. Além disso, o modo de estiramento da ligação X-H apresenta grande aumento de intensidade no espectro de infravermelho durante a formação das ligações de hidrogênio em relação ao monômero doador isolado (RUSU et al., 2011; TERRABUIO et al. 2014). Desse modo, a partição das derivadas do momento dipolar em contribuições de carga, fluxo de carga e fluxo de dipolo (CCFDF) (HAIDUKE e BRUNS, 2005), a partir dos multipolos da Teoria Quântica de Átomos em Moléculas (QTAIM - do inglês "Quantum Theory of Atoms in Molecules") (BADER, 1990), permite compreender o porquê de certas bandas vibracionais se intensificarem durante a formação de ligações de hidrogênio (TERRABUIO et al., 2014).

Para dímeros simples tais como $\mathrm{HF} \ldots \mathrm{HCl}$ e $\mathrm{HCl}$... HCl, por exemplo, observa-se que a banda correspondente ao estiramento XH torna-se mais intensa devido a formação da ligação de hidrogênio e isto ocorre em virtude da contribuição cruzada entre a carga e o fluxo de carga $\left(A^{C \times C F}\right)$ para a intensidade de infravermelho total (TERRABUIO et al., 2014; SILVA et al., 2014). As mudanças desta propriedade espectroscópica durante a dimerização destes sistemas mais simples, ou seja, contendo uma ligação de hidrogênio, já foi analisada 
em alguns trabalhos anteriores (TERRABUIO et al., 2014; SILVA et al., 2014). No entanto, uma investigação mais aprofundada do fenômeno de interação entre monômeros e aceptores de prótons formando dímeros bidentados ( $\mathrm{HCOOH} . . . \mathrm{HCOOH}, \mathrm{FCOOH} . . . \mathrm{FCOOH}$, dentre outros), isto é, com duas ligações de hidrogênio, e o seu reflexo nas intensidades de infravermelho segundo o modelo CCFDF/QTAIM (HAIDUKE e BRUNS, 2005) ainda não consta na literatura. Estes dímeros de ácidos carboxílicos apenas foram avaliados por modelos mais simples, como o modelo de carga de equilíbrio - fluxo de carga (ECCF), que não considera a assimetria na distribuição de carga eletrônica na molécula, isto é, a polarização atômica (GALIMBERTI et al., 2013).

\section{OBJETIVOS}

2.1 Analisar as variações das propriedades topológicas QTAIM e investigar a estabilização e a desestabilização das ligações de hidrogênio e das hidroxilas nos monômeros e dímeros de ácidos carboxílicos segundo a partição de Átomos Quânticos Interagentes;

2.2 Aplicar o modelo de partição de Carga - Fluxo de Carga - Fluxo de Dipolo em termos dos multipolos da Teoria Quântica de Átomos em Moléculas para compreender a natureza das variações nas intensidades de infravermelho durante a formação de ligações de hidrogênio em dímeros de ácidos carboxílicos.

\section{METODOLOGIA}

Os cálculos dos monômeros e dímeros de ácidos carboxílicos foram realizados no programa Gaussian 09 (FRISCH et al., 2013) utilizando-se o funcional duplo híbrido B2PLYP (GRIMME, 2006) com o conjunto triplo- $\zeta$ de funções de base de Dunning (1989) incluindo funções difusas, aug-cc-pVTZ (KENDALL, DUNNING JR. e HARRISON, 1992; WILSON et al., 1999; WOON e DUNNING, 1993). A primeira etapa envolveu a determinação de todas as estruturas moleculares. As energias de dimerização, as propriedades topológicas e energéticas, os momentos de dipolo, as frequências vibracionais harmônicas, as intensidades fundamentais de infravermelho e as cargas atômicas, obtidas por dois formalismos diferentes, QTAIM (BADER, 1990) e NPA (REED, WEINSTOCK e WEINHOLD, 1985), foram determinadas a partir das geometrias de equilíbrio (veja o 
esquema genérico na Figura 3). As energias de dimerização foram corrigidas pela energia do ponto zero (do inglês Zero point energy - ZPE) e os pelos erros provenientes da superposição das funções de base (do inglês Basis set superposition error - BSSE), que foram determinados por meio da metodologia "counterpoise" (BOYS, BERNARDI, 1970). Além disso, quantidades como densidade eletrônica $\left(\rho_{B C P}\right)$, Laplaciano da densidade eletrônica $\left(\nabla^{2} \rho_{B C P}\right)$ e densidade de energia eletrônica $\left(H_{b}\right)$ nos pontos críticos das ligações O...H e O-H foram avaliadas. Algumas destas propriedades topológicas, como o Laplaciano e as curvaturas da densidade eletrônica, também foram analisadas nos pontos críticos dos anéis (RCPs) presentes nos dímeros de ácidos carboxílicos, ilustrados esquematicamente na Figura 3.

Figura 3 - Estrutura dos dímeros de ácido carboxílicos plotada com o software Molden (version 5.3) (SCHAFTENAAR e NOORDIK, 2000), em que $\mathrm{X}$ e $\mathrm{X}^{\prime}$ referem a $\mathrm{H}, \mathrm{CH}_{3}, \mathrm{~F}, \mathrm{Cl}$ ou $\mathrm{Br}$ (note o anel formado após a dimerização)

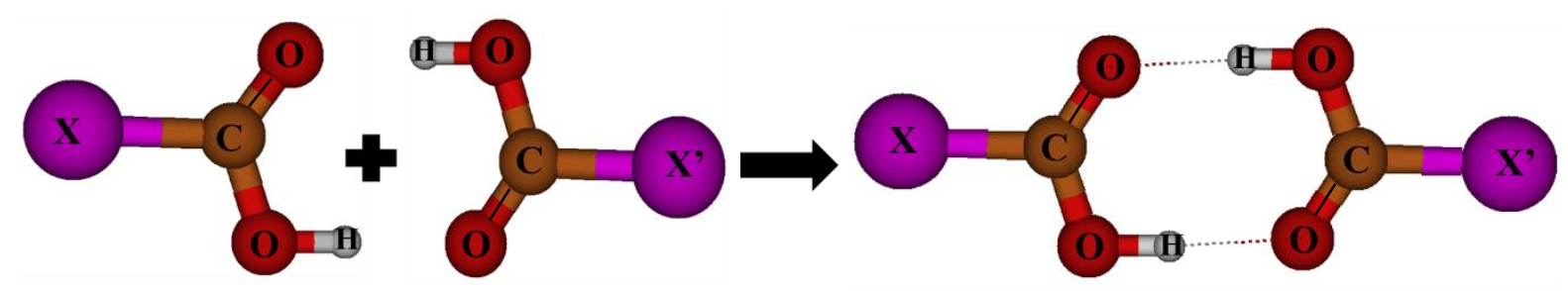

Além desta análise topológica, realizou-se um estudo da decomposição da energia eletrônica de interação das ligações de hidrogênio nos dímeros $(\mathrm{O} \ldots \mathrm{H})$ e das ligações $\mathrm{O}-\mathrm{H}$ dos monômeros e dímeros de ácidos carboxílicos segundo a partição de Átomos Quânticos Interagentes (IQA) (BLANCO, PENDÁS e FRANCISCO, 2005, 2006). Esta análise foi realizada empregando-se as densidades eletrônicas dos cálculos B3LYP/aug-cc-pVTZ nas geometrias previamente otimizadas com B2PLYP/aug-cc-pVTZ devido à alta demanda por recursos computacionais associada ao emprego do funcional duplo híbrido na realização da partição IQA completa para alguns dos sistemas estudados neste trabalho.

Para determinar as intensidades de infravermelho e as contribuições para esta quantidade de acordo com a partição CCFDF/QTAIM, os parâmetros QTAIM de carga e dipolo atômico foram obtidos com o programa AIMAll (version 16.10.31) (KEITH, 2016) nas posições de equilíbrio dos átomos e também em estruturas nas quais cada átomo foi deslocado $0,01 \AA$ a o longo dos três eixos Cartesianos. As cargas e dipolos atômicos foram, portanto, utilizados para calcular as derivadas do momento de dipolo molecular, por meio de uma abordagem numérica usual de dois pontos, e suas contribuições de carga, fluxo de carga e 
fluxo de dipolo são especificadas em coordenadas Cartesianas. Em seguida, estas derivadas foram convertidas para o sistema de coordenadas normal, que descreve os modos vibracionais, e, por fim, as intensidades de infravermelho foram estimadas pelo modelo CCFDF/QTAIM.

\section{RESULTADOS E DISCUSSÕES}

\subsection{Propriedades energéticas e topológicas das ligações de hidrogênio}

A Tabela 1 mostra as energias associadas à formação simultânea das duas ligações de hidrogênio em dímeros de ácidos carboxílicos, $E_{H B}$ (do inglês “hydrogen bond energy”). Os valores negativos desta propriedade indicam a estabilização do sistema considerado de modo que a energia do dímero $\left(E_{\mathrm{a} \ldots \mathrm{b}}\right)$ é menor que a soma das energias dos monômeros isolados $\left(E_{\mathrm{a}} e E_{b}\right)$, ou seja, $E_{H B}=E_{\mathrm{a} \ldots \mathrm{b}}-\left(E_{\mathrm{a}}+E_{b}\right)$ (MATTA e BOYD, 2007; Da SILVA e HAIDUKE, 2019). Os dados apresentados foram corrigidos pela contribuição da energia de ponto zero $\left(E_{H B(Z P E)}\right)$ e erros de superposição de funções de base.

Tabela 1 - Energias das ligações de hidrogênio $\left(E_{H B}\right)$ durante a dimerização de ácidos carboxílicos obtidas em cálculos B2PLYP/aug-cc-pVTZ ( $\left.\mathrm{kcal} \mathrm{mol}^{-1}\right)$

\begin{tabular}{|c|c|c|c|}
\hline Reação & $E_{H B}$ & $E_{H B(Z P E)}$ & $E_{H B(Z P E+B S S E)}$ \\
\hline $\mathrm{HCOOH}+\mathrm{HCOOH} \rightarrow \mathrm{HCOOH} . . . \mathrm{HCOOH}$ & $-15,97$ & $-14,05$ & $-13,43$ \\
\hline $\mathrm{FCOOH}+\mathrm{FCOOH} \rightarrow \mathrm{FCOOH} . . . \mathrm{FCOOH}$ & $-17,07$ & $-15,72$ & $-15,00$ \\
\hline $\mathrm{ClCOOH}+\mathrm{ClCOOH} \rightarrow \mathrm{ClCOOH} . . . \mathrm{ClCOOH}$ & $-15,41$ & $-14,20$ & $-13,43$ \\
\hline $\mathrm{BrCOOH}+\mathrm{BrCOOH} \rightarrow \mathrm{BrCOOH}_{. . .} \mathrm{BrCOOH}$ & $-15,03$ & $-13,90$ & $-12,98$ \\
\hline $\mathrm{ClCOOH}+\mathrm{HCOOH} \rightarrow \mathrm{ClCOOH} . . . \mathrm{HCOOH}$ & $-16,07$ & $-14,51$ & $-13,82$ \\
\hline $\mathrm{ClCOOH}+\mathrm{FCOOH} \rightarrow \mathrm{ClCOOH} . . . \mathrm{FCOOH}$ & $-16,22$ & $-14,94$ & $-14,20$ \\
\hline $\mathrm{ClCOOH}+\mathrm{BrCOOH} \rightarrow \mathrm{ClCOOH} . . . \mathrm{BrCOOH}$ & $-15,22$ & $-14,05$ & $-13,20$ \\
\hline $\mathrm{FCOOH}+\mathrm{HCOOH} \rightarrow \mathrm{FCOOH} . . . \mathrm{HCOOH}$ & $-16,96$ & $-15,34$ & $-14,67$ \\
\hline $\mathrm{FCOOH}+\mathrm{BrCOOH} \rightarrow \mathrm{FCOOH} . . . \mathrm{BrCOOH}$ & $-16,01$ & $-14,77$ & $-13,95$ \\
\hline $\mathrm{BrCOOH}+\mathrm{HCOOH} \rightarrow \mathrm{BrCOOH} . . . \mathrm{HCOOH}$ & $-15,90$ & $-14,37$ & $-13,61$ \\
\hline $\mathrm{CH}_{3} \mathrm{COOH}+\mathrm{HCOOH} \rightarrow \mathrm{CH}_{3} \mathrm{COOH} . . . \mathrm{HCOOH}$ & $-16,30$ & $-14,63$ & $-13,98$ \\
\hline $\mathrm{CH}_{3} \mathrm{COOH}+\mathrm{FCOOH} \rightarrow \mathrm{CH}_{3} \mathrm{COOH} \ldots . . \mathrm{FCOOH}$ & $-17,63$ & $-16,33$ & $-15,61$ \\
\hline $\mathrm{CH}_{3} \mathrm{COOH}+\mathrm{ClCOOH} \rightarrow \mathrm{CH}_{3} \mathrm{COOH} . . . \mathrm{ClCOOH}$ & $-16,76$ & $-15,51$ & $-14,78$ \\
\hline $\mathrm{CH}_{3} \mathrm{COOH}+\mathrm{BrCOOH} \rightarrow \mathrm{CH}_{3} \mathrm{COOH} \ldots \mathrm{BrCOOH}$ & $-16,62$ & $-15,44$ & $-14,62$ \\
\hline
\end{tabular}


Os dímeros de ácidos carboxílicos $\mathrm{CH}_{3} \mathrm{COOH}$...FCOOH, FCOOH...FCOOH e FCOOH...HCOOH são os mais estáveis $\left(E_{H B}=-17,63,-17,07\right.$ e $-16,96 \mathrm{kcal} \mathrm{mol}^{-1}$, respectivamente), enquanto os sistemas $\mathrm{BrCOOH} . . . \mathrm{BrCOOH}, \mathrm{ClCOOH} . . . \mathrm{BrCOOH}$ e НСООН...HCOOH são os menos estáveis $\left(E_{H B}=-15,03,-15,22\right.$ e $-15,97$ kcal mol ${ }^{-1}$, respectivamente), independentemente do tipo de correção aplicada nesta propriedade. Além disto, a média aritmética das duas distâncias das ligações de hidrogênio O...H presentes em cada sistema correlaciona-se de forma linear com a energia de dimerização $\left(R^{2}=0,901\right)$, como mostrado na Figura 4.

Figura 4 - Correlação entre a energia de dimerização, $E_{H B}$, e a média das distâncias de ligação $\mathrm{O}-\mathrm{H},\langle r(O \ldots H)\rangle$, segundo cálculos B2PLYP/aug-cc-pVTZ

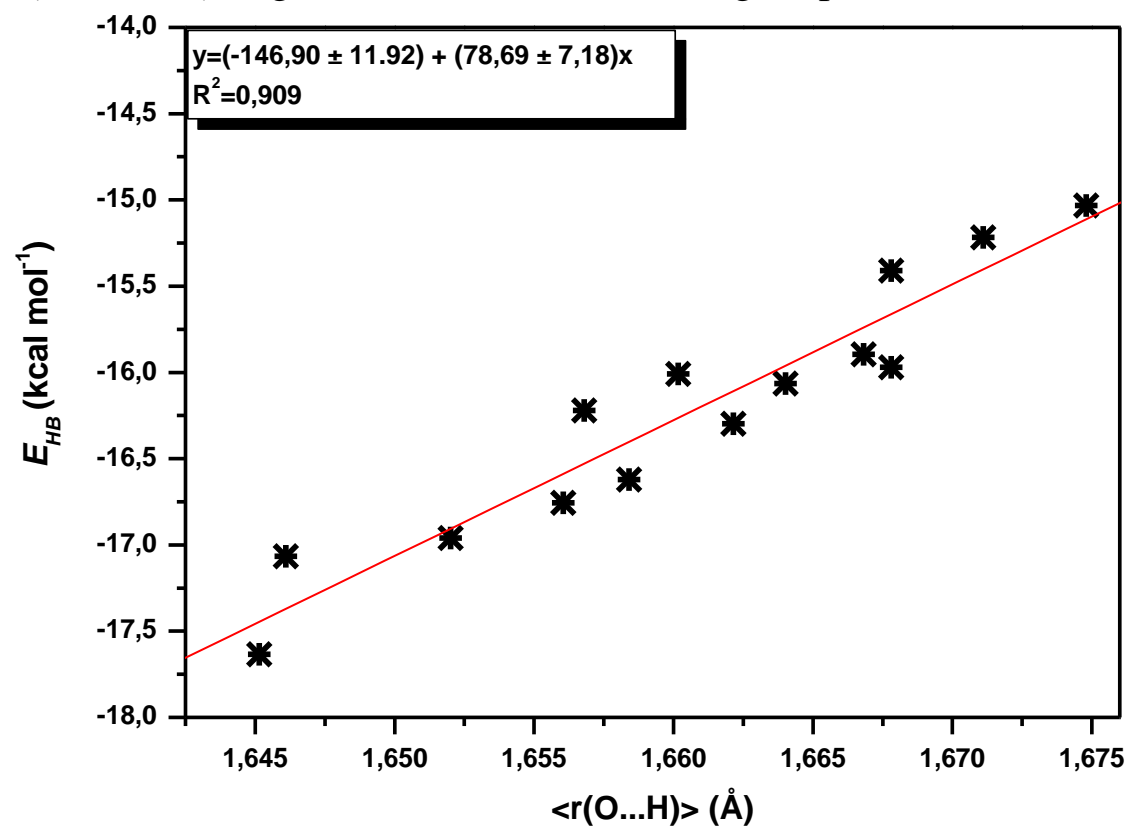

A Tabela 2 mostra as propriedades topológicas nos pontos críticos das ligações de hidrogênio dos dímeros de ácidos carboxílicos. As ligações de hidrogênio entre a carbonila $(\mathrm{C}=\mathrm{O})$ do ácido acético e a hidroxila de monômeros halogenados são aquelas que apresentam maiores valores de densidade eletrônica, $\rho_{B C P} \geq 0,060 \mathrm{u}$. a. Quando o ácido acético, por sua vez, é substituído pelo ácido fórmico, $\rho_{B C P}$ diminui para, aproximadamente, 0,056 u. a. A densidade eletrônica no ponto crítico de ligação tende a se correlacionar com a força de ligação como mostrado por Mo (2012) e Grabowski (2001) e isto sugere então que as ligações de hidrogênio mencionadas anteriormente são as mais fortes (individualmente) no conjunto de dímeros considerado. Isto poderia ser justificado pelo fato de um grupo metila, substituinte tido como doador de densidade eletrônica, estar diretamente ligado a carbonila, enquanto o halogênio parece atuar como retirador de carga eletrônica do hidrogênio da hidroxila. Por 
outro lado, os menores valores de densidade eletrônica ( $\rho_{B C P} \leq 0,046$ u. a.) ocorrem para as ligações de hidrogênio entre carbonilas de monômeros halogenados e hidroxilas do ácido fórmico e, principalmente, do ácido acético. Assim, para estes sistemas, ao mesmo tempo em que uma ligação de hidrogênio apresenta uma densidade eletrônica mais alta no ponto crítico, a outra ligação HB presente no sistema possui valores mais baixos desta propriedade.

Tabela 2 - Propriedades QTAIM nos pontos críticos das ligações de hidrogênio calculadas em nível B2PLYP/aug-cc-pVTZ (em u. a.)

\begin{tabular}{|c|c|c|c|c|c|c|c|}
\hline Sistema $^{a}$ & $\rho_{B C P}(\mathrm{O} \ldots \mathrm{H})$ & $\nabla^{2} \rho_{B C P}$ & $H_{b}=\left(\mathrm{G}_{\mathrm{b}}+\mathrm{V}_{\mathrm{b}}\right)$ & Sistema & $\rho_{B C P}\left(\mathrm{H}_{\ldots} . . \mathrm{O}\right)$ & $\nabla^{2} \rho_{B C P}$ & $H_{b}=\left(\mathrm{G}_{\mathrm{b}}+\mathrm{V}_{\mathrm{b}}\right)$ \\
\hline НСООН...НСООН & 0,0496 & 0,1002 & $-0,0128$ & НСOOH...НСОOH & 0,0496 & 0,1002 & $-0,0128$ \\
\hline FCOOH...FCOOH & 0,0510 & 0,1053 & $-0,0135$ & FCOOH...FCOOH & 0,0510 & 0,1053 & $-0,0135$ \\
\hline СlСООН...СlCOOH & 0,0484 & 0,1019 & $-0,0120$ & СlCOOH...ClCOOH & 0,0484 & 0,1019 & $-0,0120$ \\
\hline $\mathrm{BrCOOH} . . . \mathrm{BrCOOH}$ & 0,0476 & 0,1009 & $-0,0116$ & ВrCOOH...BrCOOH & 0,0476 & 0,1009 & $-0,0116$ \\
\hline FСООН...НСООН & 0,0458 & 0,1012 & $-0,0104$ & FCOOH...HCOOH & 0,0569 & 0,1033 & $-0,0173$ \\
\hline FCOOH....BrCOOH & 0,0497 & 0,1033 & $-0,0127$ & FCOOH...BrCOOH & 0,0489 & 0,1027 & $-0,0123$ \\
\hline СlСООН...НСООН & 0,0441 & 0,0993 & $-0,0095$ & ClCOOH...HCOOH & 0,0556 & 0,1022 & $-0,0165$ \\
\hline $\mathrm{ClCOOH} . . . \mathrm{FCOOH}$ & 0,0496 & 0,1034 & $-0,0127$ & СlCOOH...FCOOH & 0,0498 & 0,1038 & $-0,0128$ \\
\hline СlСООН...ВrCOOH & 0,0483 & 0,1015 & $-0,0120$ & СlCOOH...BrCOOH & 0,0477 & 0,1013 & $-0,0116$ \\
\hline $\mathrm{BrCOOH} . . . \mathrm{HCOOH}$ & 0,0434 & 0,0986 & $-0,0091$ & ВrCOOH...HCOOH & 0,0557 & 0,1018 & $-0,0166$ \\
\hline $\mathrm{CH}_{3} \mathrm{COOH} . . . \mathrm{HCOOH}$ & 0,0531 & 0,1017 & $-0,0149$ & $\mathrm{CH}_{3} \mathrm{COOH} \ldots \mathrm{HCOOH}$ & 0,0477 & 0,0995 & $-0,0116$ \\
\hline $\mathrm{CH}_{3} \mathrm{COOH} . . . \mathrm{FCOOH}$ & 0,0611 & 0,1040 & $-0,0201$ & $\mathrm{CH}_{3} \mathrm{COOH} \ldots \mathrm{FCOOH}$ & 0,0442 & 0,1002 & $-0,0095$ \\
\hline $\mathrm{CH}_{3} \mathrm{COOH} . . . \mathrm{ClCOOH}$ & 0,0601 & 0,1030 & $-0,0194$ & $\mathrm{CH}_{3} \mathrm{COOH} \ldots \mathrm{ClCOOH}$ & 0,0426 & 0,0983 & $-0,0087$ \\
\hline $\mathrm{CH}_{3} \mathrm{COOH} . . . \mathrm{BrCOOH}$ & 0,0604 & 0,1025 & $-0,0197$ & $\mathrm{CH}_{3} \mathrm{COOH} \ldots \mathrm{BrCOOH}$ & 0,0419 & 0,0975 & $-0,0083$ \\
\hline
\end{tabular}

${ }^{a}$ Os átomos destacados em negrito indicam a ligação de hidrogênio considerada.

O Laplaciano da densidade eletrônica, $\nabla^{2} \rho$, é um indicador que descreve se há um acúmulo ou não de carga eletrônica na região da ligação (MATTA e BOYD, 2007). Os dímeros bidentados possuem $\nabla^{2} \rho>0$, o que é uma característica comum das ligações de hidrogênio fracas e daquelas que possuem força média (GRABOWSKI, 2007). Além disso, a densidade de energia total no ponto crítico de ligação $\left(H_{b}\right)$ apresenta valores negativos para os sistemas estudados, indicando o predomínio da energia potencial em relação à energia cinética para as interações $\mathrm{O} . . . \mathrm{H}$, principalmente para a ligação de hidrogênio correspondente a interação da carbonila do ácido acético com a hidroxila de monômeros halogenados $\left(H_{b} \approx-\right.$ 0.020 u. a.) (Da SILVA e HAIDUKE, 2019). Isto significa que a localização da densidade eletrônica possui um impacto estabilizante no sistema (GRABOWSKI, 2011). Segundo a definição de Rozas et al. (2000), as ligações de hidrogênio presentes em dímeros de ácidos 
carboxílicos são de força média, visto que o valor absoluto da energia está entre 12 e 24 kcal $\mathrm{mol}^{-1}$ (ver Tabela 1$), \nabla^{2} \rho_{\mathrm{H} \ldots \mathrm{Y}}>0$ e $H_{b}<0$.

Conforme se observa na Tabela 3, a formação das ligações de hidrogênio acarreta na diminuição das densidades eletrônicas nos pontos críticos de ligação das hidroxilas que participam diretamente destas interações, sugerindo, por sua vez, um enfraquecimento das ligações O-H. Além disso, a Figura 5 indica que as densidades eletrônicas nos BCPs das ligações de hidrogênio são inversamente proporcionais àquelas correspondentes aos BCPs das ligações O-H. Esta tendência já foi observada para outros sistemas com HBs (SHAHI e ARUNAN, 2014). Por sua vez, os monômeros halogenados nos dímeros com ácido etanoico são os que apresentam maiores variações do Laplaciano da densidade eletrônica para as hidroxilas $(0,43,0,41$ e 0,40 u. a., respectivamente), indicando um aumento da ionicidade das ligações O-H destes monômeros durante a formação das ligações de hidrogênio. Assim como ocorre com $\nabla^{2} \rho_{B C P}$, as variações na densidade de energia total são maiores que zero e as maiores mudanças nos valores desta propriedade são as apresentadas pelos monômeros FCOOH e ClCOOH nos dímeros com ácidos acético e fórmico.

Tabela 3 - Variações nas propriedades topológicas QTAIM nos pontos críticos de ligação O$\mathrm{H}$ de dímeros de ácidos carboxílicos em relação aos monômeros isolados em suas geometrias de equilíbrio calculadas em nível B2PLYP/aug-cc-pVTZ (em u. a.)

\begin{tabular}{|c|c|c|c|c|}
\hline Sistema & Monômero & $\Delta\left[\rho_{B C \mathrm{P}}(\mathrm{O}-\mathrm{H})\right]$ & $\Delta\left[\nabla^{2} \rho_{B C P}(\mathrm{O}-\mathrm{H})\right]$ & $\Delta\left[H_{b}=\left(\mathrm{G}_{\mathrm{b}}+\mathrm{V}_{\mathrm{b}}\right)(\mathrm{O}-\mathrm{H})\right]$ \\
\hline $\mathrm{HCOOH} . . . \mathrm{HCOOH}$ & $\mathrm{HCOOH}$ & $-0,0376$ & 0,2517 & 0,0677 \\
\hline FCOOH...FCOOH & $\mathrm{FCOOH}$ & $-0,0402$ & 0,2924 & 0,0766 \\
\hline $\mathrm{ClCOOH} . . . \mathrm{ClCOOH}$ & $\mathrm{ClCOOH}$ & $-0,0360$ & 0,2517 & 0,0667 \\
\hline $\mathrm{BrCOOH} . . . \mathrm{BrCOOH}$ & $\mathrm{BrCOOH}$ & $-0,0347$ & 0,2388 & 0,0636 \\
\hline \multirow[t]{2}{*}{ FCOOH...HCOOH } & $\mathrm{FCOOH}$ & $-0,0480$ & 0,3797 & 0,0969 \\
\hline & $\mathrm{HCOOH}$ & $-0,0324$ & 0,1983 & 0,0551 \\
\hline \multirow[t]{2}{*}{ FCOOH...BrCOOH } & $\mathrm{FCOOH}$ & $-0,0378$ & 0,2736 & 0,0718 \\
\hline & $\mathrm{BrCOOH}$ & $-0,0370$ & 0,2563 & 0,0680 \\
\hline \multirow[t]{2}{*}{ СlCOOH...HCOOH } & $\mathrm{ClCOOH}$ & $-0,0453$ & 0,3480 & 0,0896 \\
\hline & $\mathrm{HCOOH}$ & $-0,0306$ & 0,1846 & 0,0516 \\
\hline \multirow[t]{2}{*}{$\mathrm{ClCOOH} . . . \mathrm{FCOOH}$} & $\mathrm{ClCOOH}$ & $-0,0375$ & 0,2632 & 0,0696 \\
\hline & $\mathrm{FCOOH}$ & $-0,0386$ & 0,2802 & 0,0735 \\
\hline \multirow[t]{2}{*}{$\mathrm{ClCOOH} . . . \mathrm{BrCOOH}$} & $\mathrm{ClCOOH}$ & $-0,0352$ & 0,2455 & 0,0652 \\
\hline & $\mathrm{BrCOOH}$ & $-0,0355$ & 0,2454 & 0,0653 \\
\hline \multirow[t]{2}{*}{ ВrCOOH...HCOOH } & $\mathrm{BrCOOH}$ & $-0,0450$ & 0,3435 & 0,0886 \\
\hline & $\mathrm{HCOOH}$ & $-0,0299$ & 0,1790 & 0,0502 \\
\hline \multirow[t]{2}{*}{$\mathrm{CH}_{3} \mathrm{COOH} . . . \mathrm{HCOOH}$} & $\mathrm{CH}_{3} \mathrm{COOH}$ & $-0,0356$ & 0,2326 & 0,0632 \\
\hline & $\mathrm{HCOOH}$ & $-0,0415$ & 0,2912 & 0,0772 \\
\hline
\end{tabular}




\begin{tabular}{llrrr}
\hline Sistema & Monômero & $\Delta\left[\rho_{B C \mathrm{P}}(\mathrm{O}-\mathrm{H})\right]$ & $\Delta\left[\nabla^{2} \rho_{B C P}(\mathrm{O}-\mathrm{H})\right]$ & $\Delta\left[H_{b}=\left(\mathrm{G}_{\mathrm{b}}+\mathrm{V}_{\mathrm{b}}\right)(\mathrm{O}-\mathrm{H})\right]$ \\
\hline $\mathrm{CH}_{3} \mathrm{COOH} . . . \mathrm{FCOOH}$ & $\mathrm{CH}_{3} \mathrm{COOH}$ & $-0,0307$ & 0,1832 & 0,0515 \\
& $\mathrm{FCOOH}$ & $-0,0531$ & 0,4343 & 0,1098 \\
$\mathrm{CH}_{3} \mathrm{COOH} . . . \mathrm{ClCOOH}$ & $\mathrm{CH}_{3} \mathrm{COOH}$ & $-0,0290$ & 0,1704 & 0,0482 \\
& $\mathrm{ClCOOH}$ & $-0,0507$ & 0,4050 & 0,1031 \\
$\mathrm{CH}_{3} \mathrm{COOH} . . . \mathrm{BrCOOH}$ & $\mathrm{CH}_{3} \mathrm{COOH}$ & $-0,0283$ & 0,1652 & 0,0696 \\
& $\mathrm{BrCOOH}$ & $-0,0506$ & 0,4030 & 0,0636 \\
\hline
\end{tabular}

Figura 5 - Correlação entre as densidades eletrônicas nos pontos críticos das ligações de hidrogênio e de hidroxilas, respectivamente $\rho_{B C P}(\mathrm{O} \ldots \mathrm{H})$ e $\rho_{B C P}(\mathrm{O}-\mathrm{H})$, de acordo com cálculos B2PLYP/aug-cc-pVTZ (em u. a.)

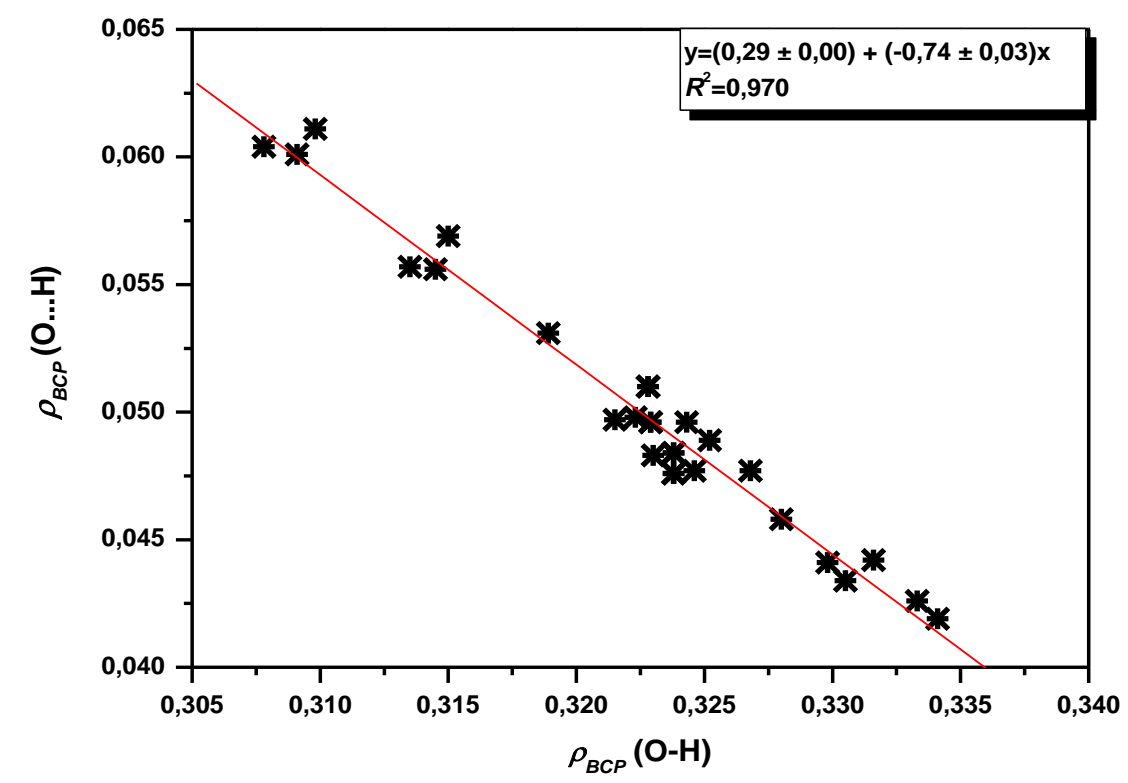

\subsection{Análise de Átomos Quânticos Interagentes (IQA)}

As energias IQA de interação entre os átomos que formam a ligação de hidrogênio, $E_{\text {Inter }}(\mathrm{O} \ldots \mathrm{H})$, são mostradas na Tabela 4. Os contatos mais fortes via ligações de hidrogênio $\left(E_{\text {Inter }} \leq-0,27\right.$ u. a.) são novamente aqueles entre a carbonila do ácido acético e as hidroxilas dos monômeros halogenados. Os termos de interação clássicos e de troca-correlação estão, respectivamente, associados com conceitos químicos de ionicidade e covalência (GUEVARA-VELA et al., 2016). Como se observa na Tabela 4, o caráter eletrostático dos contatos via ligações de hidrogênio é superior ao caráter covalente em todos os casos analisados. 
Tabela 4 - Energias de interação IQA $\left(E_{\text {Inter }}\right)$ juntamente com os componentes clássico $\left(V_{c l}\right)$ e de troca-correlação $\left(V_{X C}\right)$ para o par de átomos diretamente envolvido na ligação de hidrogênio $(\mathrm{O} \ldots \mathrm{H})$ de dímeros de ácidos carboxílicos calculados em nível B3LYP/aug-ccpVTZ (em u. a.)

\begin{tabular}{|c|c|c|c|c|c|c|c|}
\hline Sistema & $E_{\text {Inter }}(\mathrm{O} \ldots \mathrm{H})^{\mathrm{a}}$ & $V_{c l}(\mathrm{O} \ldots \mathrm{H})$ & $V_{X C}(\mathrm{O} \ldots \mathrm{H})$ & Sstema & $E_{\text {Inter }}(\mathrm{H} \ldots \mathrm{O})$ & $V_{c l}(\mathrm{H} \ldots \mathrm{O})$ & $V_{X C}(\mathrm{H} \ldots \mathrm{O})$ \\
\hline НСООН...НСООН & $-0,2513$ & $-0,2229$ & $-0,0284$ & НСООН...НСООН & $-0,2515$ & $-0,2232$ & $-0,0284$ \\
\hline FCOOH...FCOOH & $-0,2593$ & $-0,2312$ & $-0,0281$ & FCOOH...FCOOH & $-0,2595$ & $-0,2314$ & $-0,0281$ \\
\hline СlCOOH...СlCOOH & $-0,2473$ & $-0,2201$ & $-0,0272$ & СlCOOH...ClCOOH & $-0,2475$ & $-0,2203$ & $-0,0272$ \\
\hline ВrCOOH...ВrCOOH & $-0,2442$ & $-0,2174$ & $-0,0268$ & ВrCOOH...BrCOOH & $-0,2444$ & $-0,2176$ & $-0,0268$ \\
\hline FCOOH...HCOOH & $-0,2471$ & $-0,2210$ & $-0,0261$ & FCOOH...HCOOH & $-0,2661$ & $-0,2347$ & $-0,0315$ \\
\hline FCOOH...BrCOOH & $-0,2540$ & $-0,2261$ & $-0,0279$ & FCOOH...BrCOOH & $-0,2498$ & $-0,2227$ & $-0,0271$ \\
\hline СlCOOH...НСООН & $-0,2389$ & $-0,2137$ & $-0,0252$ & СlСООН...НСОOH & $-0,2619$ & $-0,2307$ & $-0,0312$ \\
\hline СlCOOН...FСООН & $-0,2517$ & $-0,2242$ & $-0,0274$ & СlСООН...FСООН & $-0,2551$ & $-0,2272$ & $-0,0279$ \\
\hline СlCOOH...BrCOOH & $-0,2464$ & $-0,2192$ & $-0,0272$ & СlCOOH...BrCOOH & $-0,2454$ & $-0,2186$ & $-0,0268$ \\
\hline ВrCOOH...НСООН & $-0,2367$ & $-0,2119$ & $-0,0248$ & ВrCOOH...НСОOH & $-0,2614$ & $-0,2300$ & $-0,0313$ \\
\hline $\mathrm{CH}_{3} \mathrm{COOH} . . . \mathrm{HCOOH}$ & $-0,2593$ & $-0,2292$ & $-0,0301$ & $\mathrm{CH}_{3} \mathrm{COOH} . . \mathrm{HCOOH}$ & $-0,2480$ & $-0,2205$ & $-0,0275$ \\
\hline $\mathrm{CH}_{3} \mathrm{COOH} \ldots \mathrm{FCOOH}$ & $-0,2749$ & $-0,2413$ & $-0,0336$ & $\mathrm{CH}_{3} \mathrm{COOH} \ldots \mathrm{FCOOH}$ & $-0,2441$ & $-0,2188$ & $-0,0253$ \\
\hline $\mathrm{CH}_{3} \mathrm{COOH} . . . \mathrm{ClCOOH}$ & $-0,2712$ & $-0,2378$ & $-0,0334$ & $\mathrm{CH}_{3} \mathrm{COOH} \ldots \mathrm{ClCOOH}$ & $-0,2358$ & $-0,2114$ & $-0,0245$ \\
\hline $\mathrm{CH}_{3} \mathrm{COOH} . . . \mathrm{BrCOOH}$ & $-0,2709$ & $-0,2372$ & $-0,0337$ & $\mathrm{CH}_{3} \mathrm{COOH} . . \mathrm{BrCOOH}$ & $-0,2337$ & $-0,2096$ & $-0,0241$ \\
\hline
\end{tabular}

a Os átomos destacados em negrito indicam a ligação de hidrogênio considerada.

Além disso, as energias de interação entre o par de átomos envolvidos diretamente na ligação de hidrogênio, O...H, correlacionam-se linearmente com as densidades eletrônicas nos seus respectivos BCPs, como mostrado na Figura 6. O aumento da densidade eletrônica no $\mathrm{BCP}$ das ligações O...H individuais resulta em valores menores de energia interatômica, indicando um aumento da atração entre os átomos envolvidos. Este tipo de correlação já foi observado para outros sistemas contendo ligações de hidrogênio (GRABOWSKI, 2001). Dentre os dímeros de ácidos carboxílicos analisados, os contatos via ligações de hidrogênio mais fortes $\left(E_{\text {Inter }} \leq-0,27\right.$ u. a.) são aqueles entre a carbonila do ácido acético e as hidroxilas dos monômeros halogenados como observado na análise topológica. 
Figura 6 - Correlação entre a energia interatômica das ligações de hidrogênio, $E_{\text {Inter }}(\mathrm{O} \ldots \mathrm{H})$, segundo cálculos B3LYP/aug-cc-pVTZ, e a densidade eletrônica nos seus respectivos BCPs, $\rho_{B C P}(\mathrm{O} \ldots \mathrm{H})$, de acordo com resultados B2PLYP/aug-cc-pVTZ (em u. a.)

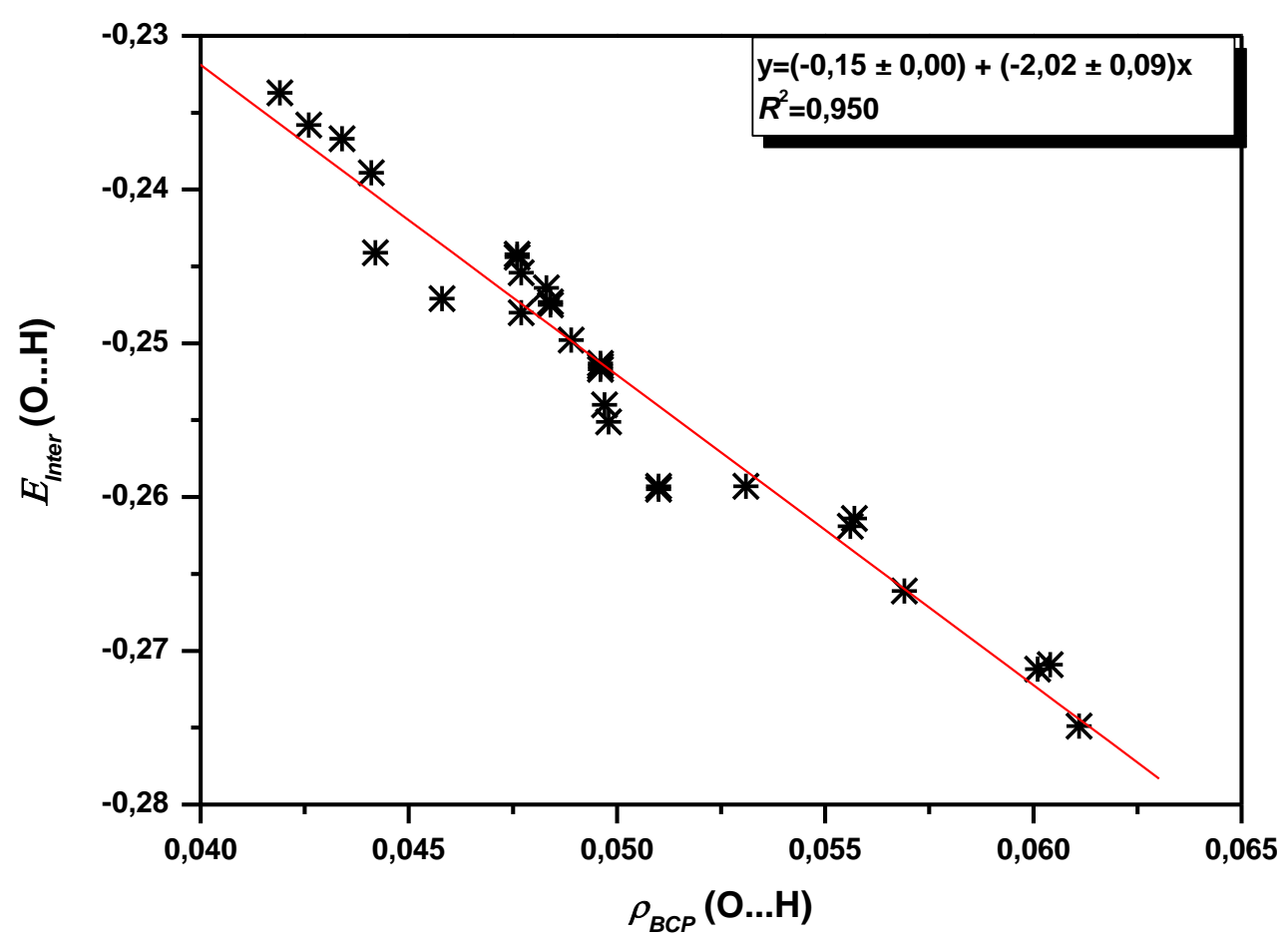

A energia de interação para as ligações O...H também se correlaciona de modo linear com o Laplaciano da densidade eletrônica nos respectivos BCPs (Figura 7). Esta propriedade topológica está usualmente correlacionada com quantidades que descrevem a energia da ligação de hidrogênio, como mostrado em estudos prévios (GRABOWSKI, 2001). No caso dos dímeros de ácidos carboxílicos, observa-se ainda uma separação dos dímeros em dois grupos, em que um deles apresenta sempre ácido fórmico ou o ácido acético como um dos monômeros enquanto o outro grupo é constituído apenas por monômeros halogenados. 
Figura 7 - Correlação entre as energias de interação interatômica da ligação de hidrogênio, $E_{\text {Inter }}(\mathrm{O} \ldots \mathrm{H})$, obtidas a partir de cálculos B3LYP/aug-cc-pVTZ, e o Laplaciano da densidade eletrônica nos respectivos BCPs, $\nabla^{2} \rho_{B C P}(\mathrm{O} \ldots \mathrm{H})$, segundo cálculos B2PLYP/aug-cc-pVTZ (em u. a.)

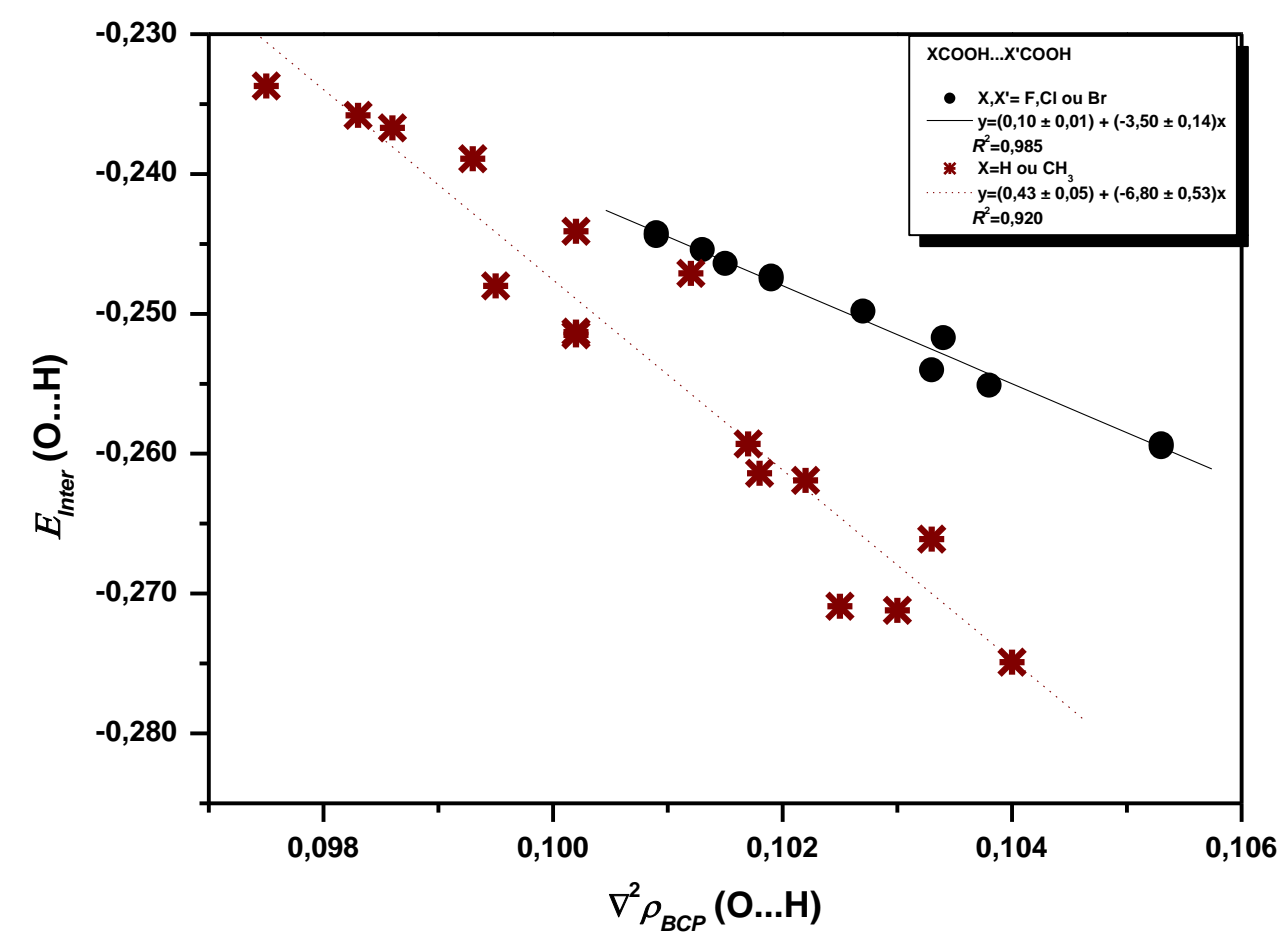

A Tabela 5 mostra as variações nos termos de energia interatômica para as ligações O-H durante a dimerização. A contribuição eletrostática diminui e a contribuição de trocacorrelação aumenta devido à formação das ligações de hidrogênio, porém $\Delta V_{c l}$ é predominante para a variação de energia de interação $\mathrm{O}-\mathrm{H}$, indicando um aumento da ionicidade das ligações hidroxilas. Pendás e colaboradores (2006) já haviam mostrado esta característica das ligações envolvidas diretamente no processo.

Tabela 5 - Variações na energia de interação IQA $\left(\Delta E_{\text {Inter }}\right)$ e nos componentes clássico $\left(\Delta V_{c l}\right)$ e de troca-correlação $\left(\Delta V_{X C}\right)$ das ligações $\mathrm{O}-\mathrm{H}$ durante a dimerização em nível de cálculo B3LYP/aug-cc-pVTZ (em u. a.)

\begin{tabular}{llccc}
\hline Sistema & Monômero & $\Delta E_{\text {Inter }}(\mathrm{O}-\mathrm{H})$ & $\Delta V_{c l}(\mathrm{O}-\mathrm{H})$ & $\Delta V_{X C}(\mathrm{O}-\mathrm{H})$ \\
\hline $\mathrm{HCOOH} . . . \mathrm{HCOOH}$ & $\mathrm{HCOOH}$ & $-0,0162$ & $-0,0631$ & 0,0469 \\
FCOOH...FCOOH & FCOOH & $-0,0123$ & $-0,0586$ & 0,0462 \\
$\mathrm{ClCOOH} . . . \mathrm{ClCOOH}$ & $\mathrm{ClCOOH}$ & $-0,0127$ & $-0,0565$ & 0,0438 \\
BrCOOH...BrCOOH & $\mathrm{BrCOOH}$ & $-0,0130$ & $-0,0560$ & 0,0430 \\
FCOOH...HCOOH & FCOOH & $-0,0081$ & $-0,0578$ & 0,0497 \\
& $\mathrm{HCOOH}$ & $-0,0176$ & $-0,0618$ & 0,0441 \\
FCOOH...BrCOOH & FCOOH & $-0,0113$ & $-0,0553$ & 0,0441 \\
& BrCOOH & $-0,0142$ & $-0,0593$ & 0,0452
\end{tabular}




\begin{tabular}{|c|c|c|c|c|}
\hline Sistema & Monômero & $\Delta E_{\text {Inter }}(\mathrm{O}-\mathrm{H})$ & $\Delta V_{c l}(\mathrm{O}-\mathrm{H})$ & $\Delta V_{X C}(\mathrm{O}-\mathrm{H})$ \\
\hline \multirow[t]{2}{*}{ СlСOOH...НСООН } & $\mathrm{ClCOOH}$ & $-0,0097$ & $-0,0587$ & 0,0490 \\
\hline & $\mathrm{HCOOH}$ & $-0,0168$ & $-0,0592$ & 0,0424 \\
\hline \multirow[t]{2}{*}{$\mathrm{ClCOOH} . . . \mathrm{FCOOH}$} & $\mathrm{ClCOOH}$ & $-0,0135$ & $-0,0588$ & 0,0453 \\
\hline & $\mathrm{FCOOH}$ & $-0,0116$ & $-0,0563$ & 0,0448 \\
\hline \multirow[t]{2}{*}{$\mathrm{ClCOOH} . . . \mathrm{BrCOOH}$} & $\mathrm{ClCOOH}$ & $-0,0124$ & $-0,0554$ & 0,0431 \\
\hline & $\mathrm{BrCOOH}$ & $-0,0133$ & $-0,0570$ & 0,0437 \\
\hline \multirow[t]{2}{*}{ ВrCOOH...HCOOH } & $\mathrm{BrCOOH}$ & $-0,0102$ & $-0,0593$ & 0,0491 \\
\hline & $\mathrm{HCOOH}$ & $-0,0164$ & $-0,0580$ & 0,0417 \\
\hline \multirow[t]{2}{*}{$\mathrm{CH}_{3} \mathrm{COOH} . . . \mathrm{HCOOH}$} & $\mathrm{CH}_{3} \mathrm{COOH}$ & $-0,0174$ & $-0,0630$ & 0,0456 \\
\hline & $\mathrm{HCOOH}$ & $-0,0156$ & $-0,0652$ & 0,0495 \\
\hline \multirow[t]{2}{*}{$\mathrm{CH}_{3} \mathrm{COOH} . . . \mathrm{FCOOH}$} & $\mathrm{CH}_{3} \mathrm{COOH}$ & $-0,0186$ & $-0,0614$ & 0,0429 \\
\hline & $\mathrm{FCOOH}$ & $-0,0064$ & $-0,0588$ & 0,0525 \\
\hline \multirow[t]{2}{*}{$\mathrm{CH}_{3} \mathrm{COOH} . . . \mathrm{ClCOOH}$} & $\mathrm{CH}_{3} \mathrm{COOH}$ & $-0,0176$ & $-0,0587$ & 0,0411 \\
\hline & $\mathrm{ClCOOH}$ & $-0,0079$ & $-0,0600$ & 0,0521 \\
\hline \multirow[t]{2}{*}{$\mathrm{CH}_{3} \mathrm{COOH} . . \mathrm{BrCOOH}$} & $\mathrm{CH}_{3} \mathrm{COOH}$ & $-0,0171$ & $-0,0575$ & 0,0404 \\
\hline & $\mathrm{BrCOOH}$ & $-0,0082$ & $-0,0605$ & 0,0522 \\
\hline
\end{tabular}

A Tabela 6, por sua vez, mostra os valores do Laplaciano da densidade eletrônica nos pontos críticos dos anéis presentes nos dímeros (veja Figura 3), assim como as três curvaturas de $\rho_{R C P}, \lambda_{1}, \lambda_{2}$ e $\lambda_{3}$. Para os homodímeros ( $\mathrm{XCOOH} . . . \mathrm{XCOOH}$, na qual $\mathrm{X}=\mathrm{H}, \mathrm{Br}, \mathrm{Cl}$ e F), o valor de $\nabla^{2} \rho_{R C P}$ tende a aumentar de acordo com a eletronegatividade do substituinte $(0,0302$ a 0,0326 u. a.). Esta mesma tendência é observada para as séries de heterodímeros $\left(\mathrm{XCOOH} . . . \mathrm{X}^{\prime} \mathrm{COOH}\right.$, na qual $\mathrm{X}$ e $\mathrm{X}^{\prime}=\mathrm{H}, \mathrm{CH}_{3}, \mathrm{Br}, \mathrm{Cl}$ e $\mathrm{F}$ e $\left.\mathrm{X} \neq \mathrm{X}^{\prime}\right)$, em que um dos monômeros é mantido constante. Além disto, a terceira curvatura da densidade eletrônica é predominante para determinar o valor do Laplaciano, enquanto a primeira e a segunda curvaturas se cancelam parcialmente.

Tabela 6 - Laplaciano e as três curvaturas da densidade eletrônica nos pontos críticos dos anéis (RCP), $\nabla^{2} \rho_{R C P}$ e $\lambda$, respectivamente, dos dímeros de ácidos carboxílicos, obtidos a partir de cálculos B2PL-YP/aug-cc-pVTZ (em u. a.)

\begin{tabular}{lrrrr}
\hline Sistema & $\nabla^{2} \rho_{R C P}$ & $\lambda_{1}$ & $\lambda_{2}$ & $\lambda_{3}$ \\
\hline BrCOOH...BrCOOH & 0,0310 & $-0,0067$ & 0,0117 & 0,0252 \\
ClCOOH...ClCOOH & 0,0315 & $-0,0066$ & 0,0102 & 0,0289 \\
FCOOH...FCOOH & 0,0326 & $-0,0067$ & 0,0102 & 0,0280 \\
HCOOH...HCOOH & 0,0302 & $-0,0066$ & 0,0100 & 0,0277 \\
FCOOH...HCOOH & 0,0314 & $-0,0067$ & 0,0111 & 0,0264 \\
FCOOH...BrCOOH & 0,0318 & $-0,0066$ & 0,0102 & 0,0285 \\
ClCOOH...HCOOH & 0,0309 & $-0,0066$ & 0,0101 & 0,0278 \\
ClCOOH...FCOOH & 0,0321 & $-0,0066$ & 0,0112 & 0,0268
\end{tabular}




\begin{tabular}{lrrrr}
\hline Sistema & $\nabla^{2} \rho_{R C P}$ & $\lambda_{1}$ & $\lambda_{2}$ & $\lambda_{3}$ \\
\hline $\mathrm{ClCOOH} . . . \mathrm{BrCOOH}$ & 0,0313 & $-0,0066$ & 0,0101 & 0,0283 \\
$\mathrm{BrCOOH} . . . \mathrm{HCOOH}$ & 0,0306 & $-0,0066$ & 0,0110 & 0,0262 \\
$\mathrm{CH}_{3} \mathrm{COOH} . . . \mathrm{HCOOH}$ & 0,0310 & $-0,0069$ & 0,0120 & 0,0259 \\
$\mathrm{CH}_{3} \mathrm{COOH} . . . \mathrm{FCOOH}$ & 0,0322 & $-0,0068$ & 0,0118 & 0,0273 \\
$\mathrm{CH}_{3} \mathrm{COOH} . . \mathrm{ClCOOH}$ & 0,0317 & $-0,0068$ & 0,0116 & 0,0269 \\
$\mathrm{CH}_{3} \mathrm{COOH} . . . \mathrm{BrCOOH}$ & 0,0315 & $-0,0068$ & 0,0116 & 0,0267 \\
\hline
\end{tabular}

A correlação entre a energia de dimerização e o Laplaciano da densidade eletrônica nos RCPs é linear para os dímeros de ácidos carboxílicos, como mostrado na Figura 8, e, novamente, se observa uma separação em grupos entre os dímeros halogenados e aquelas em que um dos monômeros é o ácido fórmico ou ácido acético. $\mathrm{O} \nabla^{2} \rho_{R C P}$, segundo Grabowski (2002), pode ser considerado um descritor da energia de ligações de hidrogênio intramoleculares. Além disso, Rybarczyc-Pirek et al. (2002) e Gora et al. (2005) mostraram que a densidade eletrônica no RCP frequentemente está correlacionada com a energia das ligações HBs intra- ou intermoleculares.

Figura 8 - Correlação entre a energia de dimerização, $E_{H B}$, e o Laplaciano da densidade eletrônica nos pontos críticos dos anéis, $\nabla^{2} \rho_{R C P}$, para dímeros de ácidos carboxílicos segundo cálculos B2PLYP/aug-cc-pVTZ (em u. a.)

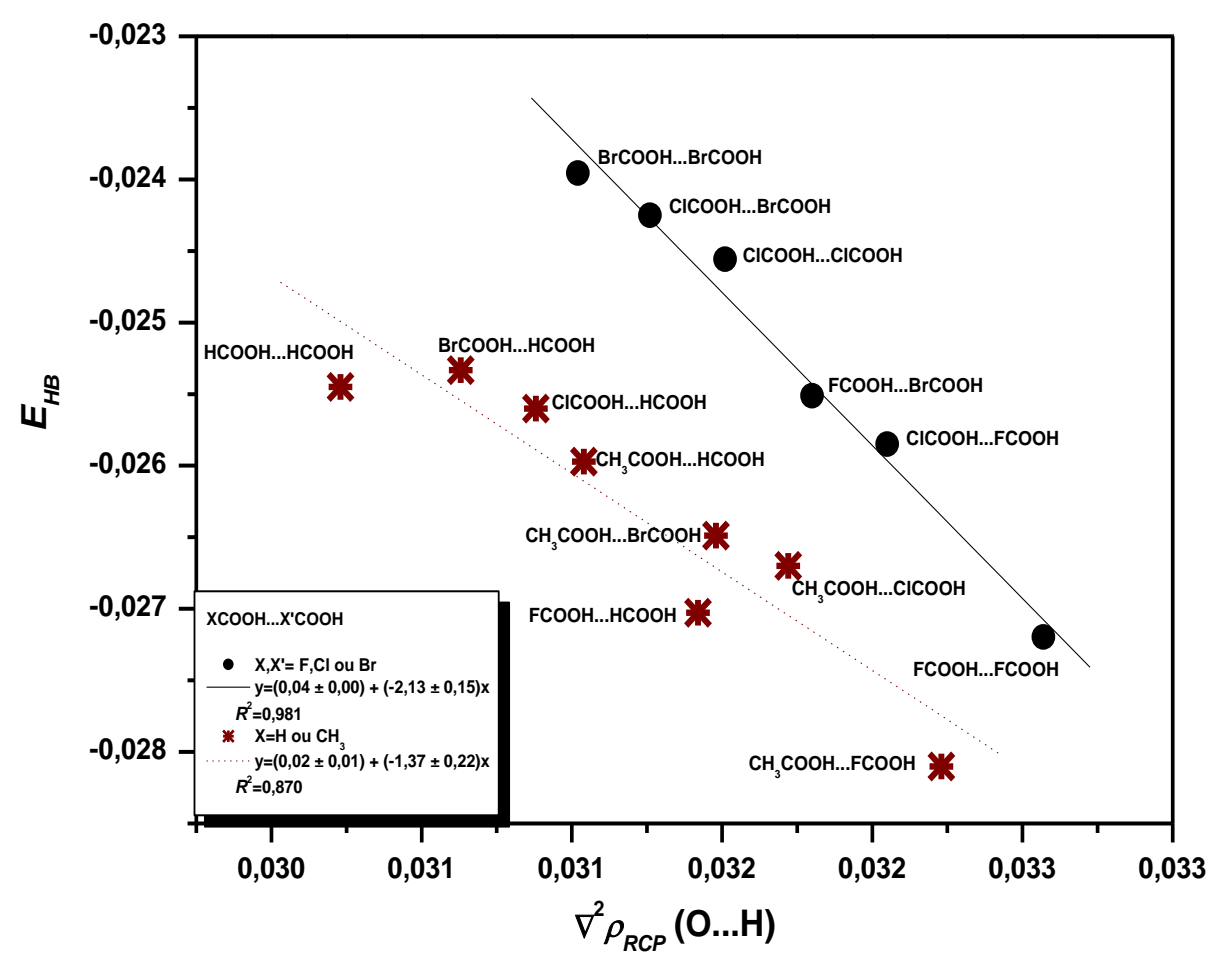




\subsection{Momentos de dipolo e propriedades espectroscópicas dos monômeros e dímeros de ácidos carboxílicos}

As magnitudes dos momentos de dipolo para os monômeros e os dímeros de ácidos carboxílicos constam na Tabela 7. As conformações dos monômeros de ácidos carboxílicos que apresentaram menores valores de energia eletrônica foram aquelas em que o oxigênio da carbonila e o hidrogênio da hidroxila estão na posição cis (veja Figura 3), de modo que esta conformação foi adotada no decorrer deste trabalho (Da SILVA e HAIDUKE, 2019). Os únicos dados experimentais encontrados referem-se ao ácido fórmico $(1,425 \pm 0,002) \mathrm{D}$ e ao ácido acético (1,70 \pm 0,02) D (HAYNES, 2016, KRISHER e SAEGEBARTH, 1971). Nestes casos, os valores teóricos de momento de dipolo diferem em somente 0,08 e 0,05 D, respectivamente. Os homodímeros possuem momento de dipolo nulo por apresentarem um centro de inversão e, por isso, não foram incluídos na Tabela 7.

Tabela 7 - Magnitude do momento de dipolo molecular, $\vec{\mu}$, para os monômeros e dímeros bidentados (em D)

\begin{tabular}{lc}
\hline Sistema & $|\vec{\mu}|$ (B2PLYP/aug-cc-pVTZ) \\
\hline HCOOH & 1,508 \\
FCOOH & 2,222 \\
ClCOOH & 1,873 \\
BrCOOH & 1,822 \\
$\mathrm{CH}_{3} \mathrm{COOH}$ & 1,747 \\
FCOOH...HCOOH & 2,005 \\
FCOOH...BrCOOH & 0,465 \\
ClCOOH...HCOOH & 1,730 \\
ClCOOH...FCOOH & 0,419 \\
ClCOOH...BrCOOH & 0,040 \\
BrCOOH...HCOOH & 1,748 \\
CH ${ }_{3} \mathrm{COOH} . . . \mathrm{HCOOH}$ & 0,988 \\
$\mathrm{CH}_{3} \mathrm{COOH} . . . \mathrm{FCOOH}$ & 3,078 \\
$\mathrm{CH}_{3} \mathrm{COOH} . . . \mathrm{ClCOOH}$ & 2,868 \\
$\mathrm{CH}_{3} \mathrm{COOH} . . . \mathrm{BrCOOH}$ & 2,921 \\
\hline
\end{tabular}

De modo geral, observa-se um aumento de 1,51 a 2,22 D no valor desta grandeza na série monomérica $\mathrm{HCOOH}<\mathrm{CH}_{3} \mathrm{COOH}<\mathrm{BrCOOH}<\mathrm{ClCOOH}<\mathrm{FCOOH}$ de acordo com a mudança do hidrogênio para substituintes mais eletronegativos. Além disso, os dímeros formados pelo ácido etanoico/ácido fórmico e pelos monômeros halogenados apresentam maiores valores de momento de dipolo $\left(\mathrm{CH}_{3} \mathrm{COOH} \ldots \mathrm{XCOOH}: 2,92\right.$ a 3,08 $\mathrm{D}$ e HCOOH...XCOOH: 1,73 a 2,01 D, em que $\mathrm{X}=\mathrm{F}, \mathrm{Cl}$ e Br). O aumento do momento de dipolo para estes dímeros, em que $\mathrm{CH}_{3} \mathrm{COOH}$ e $\mathrm{HCOOH}$ foram mantidos constantes, apresentam a 
mesma sequência observada para os monômeros isolados, porém há uma inversão na ordem dos substituintes bromo e cloro.

A Tabela 8 mostra as frequências harmônicas e as intensidades de infravermelho para os estiramentos $\mathrm{O}-\mathrm{H}$ dos monômeros de ácidos carboxílicos. Neste estudo, analisaremos apenas estes estiramentos, pois são tais ligações que atuam como doadoras de próton para o estabelecimento das ligações intermoleculares de modo que suas propriedades topológicas e espectroscópicas são afetadas substancialmente. Uma das características da ligação de hidrogênio, observada previamente na literatura, é justamente a intensificação da banda correspondente ao estiramento da ligação H-X do monômero doador (TERRABUIO et al., 2014; SILVA et al., 2014). A frequência e a intensidade de infravermelho teóricas do monômero de ácido fórmico concordam satisfatoriamente com as obtidas experimentalmente em fase gasosa, $3569 \mathrm{~cm}^{-1}$ e 60,6 $\mathrm{km} \mathrm{mol}^{-1}$ (YOKOYAMA, MIWA e MACHIDA, 1991), apresentando desvios de 5 e $11 \%$ apenas. Além disso, a frequência teórica para o ácido acético difere em $5 \%$ quando comparada ao dado experimental $\left(3583 \mathrm{~cm}^{-1}\right)$ (HAURIE e NOVAK, 1965).

Tabela 8 - Frequências vibracionais $\left(\mathrm{cm}^{-1}\right)$ e intensidades fundamentais de infravermelho $\left(\mathrm{km} \mathrm{mol}^{-1}\right)$ dos modos de estiramento $\mathrm{O}-\mathrm{H}$ dos monômeros isolados, obtidas a partir do nível de cálculo B2PLYP/aug-cc-pVTZ e calculadas por meio do modelo CCFDF/QTAIM

\begin{tabular}{ccrr}
\hline \multirow{2}{*}{ Sistema } & \multicolumn{2}{c}{ B2PLYP/aug-cc-pVTZ } & CCFDF/QTAIM \\
\cline { 2 - 3 } & Freq. & Int. & Int. \\
\hline HCOOH & 3733 & 67,5 & 67,3 \\
FCOOH & 3790 & 111,1 & 111,8 \\
$\mathrm{ClCOOH}$ & 3739 & 98,6 & 97,7 \\
$\mathrm{BrCOOH}$ & 3719 & 91,1 & 91,3 \\
$\mathrm{CH}_{3} \mathrm{COOH}$ & 3751 & 66,5 & 66,0 \\
\hline
\end{tabular}

As intensidades obtidas pelo modelo CCFDF/QTAIM para os monômeros isolados apresentam ótima concordância com aquelas calculadas diretamente em nível B2PLYP/augcc-pVTZ, visto que a maior diferença, $0,8 \mathrm{~km} \mathrm{~mol}^{-1}$, é obtida para o $\mathrm{ClCOOH}$. Além disto, as frequências vibracionais e as intensidades de infravermelho aumentam de acordo com a eletronegatividade do halogênio segundo a sequência $\mathrm{BrCOOH}<\mathrm{ClCOOH}<\mathrm{FCOOH}$, enquanto os valores destas quantidades para o ácido etanoico e para o ácido fórmico são bem próximas (3751 e $3733 \mathrm{~cm}^{-1}$ e 66,5 e $67,5 \mathrm{~km} \mathrm{~mol}^{-1}$, respectivamente) (Da SILVA e HAIDUKE, 2019).

A dimerização com formação de ligações de hidrogênio provoca um deslocamento batocrômico na posição da banda de absorção O-H, conforme mostrado na Tabela 9. Os 
resultados experimentais para o modo de estiramento $\mathrm{O}-\mathrm{H}$ assimétrico do ácido fórmico mostram um deslocamento de $459 \mathrm{~cm}^{-1}$ para comprimentos de onda maiores (YOKOYAMA, MIWA e MACHIDA, 1991), enquanto os cálculos teóricos evidenciam uma variação de 524 $\mathrm{cm}^{-1}$. Por sua vez, a intensidade de infravermelho experimental para o dímero HCOOH...HCOOH (1980 km mol ${ }^{-1}$ ) (YOKOYAMA, MIWA e MACHIDA, 1991) difere em somente $9 \%$ do resultado obtido em nível B2PLYP/aug-cc-pVTZ $\left(2157 \mathrm{~km} \mathrm{~mol}^{-1}\right)$.

Tabela 9 - Frequências vibracionais $\left(\mathrm{cm}^{-1}\right)$ e intensidades fundamentais de infravermelho $\left(\mathrm{km} \mathrm{mol}^{-1}\right)$ dos modos de estiramento O-H dos homo- e heterodímeros de ácidos carboxílicos, calculadas diretamente em nível de cálculo B2PLYP/aug-cc-pVTZ e obtidas por meio do modelo CCFDF/QTAIM

\begin{tabular}{|c|c|c|c|c|}
\hline \multirow{2}{*}{ Sistema } & \multirow{2}{*}{$\begin{array}{l}\text { Modo } \\
\text { vibracional }^{\text {a }}\end{array}$} & \multicolumn{2}{|c|}{ B2PLYP/aug-cc-pVTZ } & \multirow{2}{*}{$\begin{array}{r}\text { CCFDF/QTAIM } \\
\text { Int. }\end{array}$} \\
\hline & & Freq. & Int. & \\
\hline \multirow{2}{*}{ HCOOH...HCOOH } & $\mathrm{OH}(\mathrm{s})$ & 3091 & 0,0 & 0,1 \\
\hline & $\mathrm{OH}$ (as) & 3209 & 2157,3 & 2152,4 \\
\hline \multirow[t]{2}{*}{ FCOOH...FCOOH } & $\mathrm{OH}(\mathrm{s})$ & 3127 & 0,0 & 0,0 \\
\hline & $\mathrm{OH}$ (as) & 3238 & 2589,4 & 2600,1 \\
\hline \multirow[t]{2}{*}{ СlCOOH...ClCOOH } & $\mathrm{OH}(\mathrm{s})$ & 3136 & 0,0 & 0,2 \\
\hline & $\mathrm{OH}$ (as) & 3235 & 3501,6 & 3508,0 \\
\hline \multirow[t]{2}{*}{$\mathrm{BrCOOH} . . \mathrm{BrCOOH}$} & $\mathrm{OH}(\mathrm{s})$ & 3138 & 0,0 & 1,0 \\
\hline & $\mathrm{OH}$ (as) & 3232 & 3937,2 & 3857,5 \\
\hline \multirow[t]{2}{*}{ FCOOH...HCOOH } & $\mathrm{OH}(\mathrm{s})$ & 3026 & 926,3 & 918,8 \\
\hline & $\mathrm{OH}$ (as) & 3260 & 1652,3 & 1632,1 \\
\hline \multirow[t]{2}{*}{ FCOOH...BrCOOH } & $\mathrm{OH}(\mathrm{s})$ & 3125 & 200,4 & 210,4 \\
\hline & $\mathrm{OH}$ (as) & 3243 & 3030,8 & 3046,8 \\
\hline \multirow[t]{2}{*}{ СlCOOH...HCOOH } & $\mathrm{OH}(\mathrm{s})$ & 3016 & 1227,6 & 1227,1 \\
\hline & $\mathrm{OH}$ (as) & 3280 & 1798,4 & 1805,6 \\
\hline \multirow[t]{2}{*}{$\mathrm{ClCOOH} . . . \mathrm{FCOOH}$} & $\mathrm{OH}(\mathrm{s})$ & 3129 & 73,2 & 70,6 \\
\hline & $\mathrm{OH}$ (as.) & 3240 & 2955,9 & 2985,4 \\
\hline \multirow[t]{2}{*}{ СlCOOH...BrCOOH } & $\mathrm{OH}(\mathrm{s})$ & 3136 & 51,8 & 42,2 \\
\hline & $\mathrm{OH}$ (as) & 3235 & 3668,0 & 3618,2 \\
\hline \multirow[t]{2}{*}{ ВrCOOH...HCOOH } & $\mathrm{OH}(\mathrm{s})$ & 3002 & 1400,8 & 1391,1 \\
\hline & $\mathrm{OH}(\mathrm{as})$ & 3289 & 1843,6 & 1853,0 \\
\hline \multirow[t]{2}{*}{$\mathrm{CH}_{3} \mathrm{COOH} . . . \mathrm{HCOOH}$} & $\mathrm{OH}(\mathrm{s})$ & 3055 & 695,3 & 690,0 \\
\hline & $\mathrm{OH}(\mathrm{as})$ & 3225 & 2174,0 & 2137,8 \\
\hline \multirow[t]{2}{*}{$\mathrm{CH}_{3} \mathrm{COOH} . . . \mathrm{FCOOH}$} & $\mathrm{OH}(\mathrm{s})$ & 2944 & 1431,6 & 1482,4 \\
\hline & $\mathrm{OH}$ (as) & 3300 & 1604,2 & 1581,6 \\
\hline \multirow{2}{*}{$\mathrm{CH}_{3} \mathrm{COOH} . . . \mathrm{ClCOOH}$} & $\mathrm{OH}(\mathrm{s})$ & 2926 & 1784,5 & 1735,8 \\
\hline & $\mathrm{OH}$ (as) & 3321 & 1766,7 & 1834,0 \\
\hline \multirow[t]{2}{*}{$\mathrm{CH}_{3} \mathrm{COOH} . . . \mathrm{BrCOOH}$} & $\mathrm{OH}(\mathrm{s})$ & 2907 & 1986,5 & 2027,5 \\
\hline & $\mathrm{OH}$ (as) & 3330 & 1828,1 & 1834,0 \\
\hline
\end{tabular}

A maior diferença entre as intensidades de infravermelho obtidas pela partição CCFDF/QTAIM e aquelas calculadas em nível B2PLYP/aug-cc-pVTZ é observada para o homodímero de bromo $\left(79,7 \mathrm{~km} \mathrm{~mol}^{-1}\right)$. A comparação entre estas duas abordagens evidencia desvios acima de $50 \mathrm{~km} \mathrm{~mol}^{-1}$ para bandas mais intensas (pelo menos, cerca de $1500 \mathrm{~km} \mathrm{~mol}^{-}$ 
${ }^{1}$ ) devido a pequenos erros de integração dos multipolos QTAIM ou nas derivadas numéricas usadas para o cálculo de fluxos de carga e de dipolo atômico durante a determinação das intensidades CCFDF/QTAIM (Da SILVA e HAIDUKE, 2019). Entretanto, como as intensidades das bandas vibracionais consideradas são elevadas, este erro, de 3,8\% na maioria dos casos, não chega a ser significativo. Além disso, as pequenas discrepâncias numéricas também explicam o fato de os valores desta propriedade espectroscópica provenientes da partição CCFDF/QTAIM não serem exatamente zero para os modos de estiramento O-H simétricos dos homodímeros.

De modo geral, o fenômeno de dimerização é acompanhado por um aumento das intensidades de infravermelho correspondentes ao estiramento da hidroxila. As bandas vibracionais dos estiramentos $\mathrm{O}-\mathrm{H}$ assimétricos dos homodímeros e dos dímeros em que ambos os substituintes são halogênios se tornam mais intensas (2157 a 3937 e 2956 a 3668 $\mathrm{km} \mathrm{mol}^{-1}$, respectivamente) que as dos demais sistemas.

A partição das intensidades de infravermelho, segundo o modelo CCFDF/QTAIM, para os modos de estiramento $\mathrm{O}-\mathrm{H}$ em termos das contribuições de carga, fluxo de carga, fluxo de dipolo e de seus termos cruzados são encontradas na Tabela 10. Entretanto, os estiramentos $\mathrm{O}-\mathrm{H}$ simétricos e assimétricos se acoplam durante a vibração desta ligação nos heterodímeros, o que inviabiliza a correta distinção entre estes modos e, consequentemente, uma análise isolada deles. Assim, para efetuar uma análise mais apropriada, as variações das intensidades de infravermelho foram calculadas subtraindo-se a soma das intensidades dos modos de estiramento simétricos e assimétricos dos dímeros em relação àquela dos monômeros isolados $a$ e $b$ envolvidos na ligação de hidrogênio, $\Delta A=\left[\left(A_{O-H}(s)+\right.\right.$ $\left.\left.A_{O-H}(a s)\right)_{\text {Dímero }}-\left(A_{O-H}(a)+A_{O-H}(b)\right)_{\text {Monômeros }}\right]$ (Da SILVA e HAIDUKE, 2019).

Tabela 10 - Variações das contribuições de carga, fluxo de carga, fluxo de dipolo e os termos cruzados entre estas quantidades durante a dimerização para a intensidade total, $A,\left(\mathrm{~km} \mathrm{~mol}^{-1}\right)$ dos modos de estiramento O-H segundo o modelo CCFDF/QTAIM

\begin{tabular}{lccccccc}
\hline \multirow{2}{*}{ Sistema } & \multicolumn{7}{c}{ CCFDF/QTAIM $^{\mathrm{a}}$} \\
\cline { 2 - 8 } & $\Delta A^{C}$ & $\Delta A^{C F}$ & $\Delta \mathrm{A}^{D F}$ & $\Delta A^{C \times C F}$ & $\Delta A^{C \times D F}$ & $\Delta A^{C F \times D F}$ & $\Delta A^{\text {Total }}$ \\
\hline HCOOH...HCOOH & 90,0 & $-469,1$ & $-32,9$ & 2222,1 & $-191,3$ & 399,1 & 2017,8 \\
FCOOH...FCOOH & 123,8 & $-405,1$ & 80,3 & 1834,3 & 350,5 & 392,7 & 2376,6 \\
ClCOOH...ClCOOH & 127,2 & $-239,9$ & 65,1 & 2450,9 & 276,9 & 632,6 & 3312,8 \\
BrCOOH...BrCOOH & 126,1 & $-159,0$ & 59,3 & 2672,1 & 249,3 & 727,9 & 3675,9 \\
FCOOH...HCOOH & 144,8 & $-458,3$ & 50,6 & 1972,6 & 216,7 & 445,3 & 2371,8 \\
FCOOH...BrCOOH & 124,9 & $-299,2$ & 69,1 & 2293,2 & 294,8 & 571,3 & 3054,1 \\
ClCOOH...HCOOH & 150,3 & $-382,1$ & 48,7 & 2288,8 & 196,9 & 565,0 & 2867,6 \\
ClCOOH...FCOOH & 125,2 & $-337,5$ & 74,1 & 2152,4 & 315,5 & 516,8 & 2846,6
\end{tabular}




\begin{tabular}{lccccccc}
\hline \multirow{2}{*}{ Sistema } & \multicolumn{7}{c}{ CCFDF/QTAIM $^{\mathrm{a}}$} \\
\cline { 2 - 8 } & $\Delta A^{C}$ & $\Delta A^{C F}$ & $\Delta \mathrm{A}^{D F}$ & $\Delta A^{C \times C F}$ & $\Delta A^{C \times D F}$ & $\Delta A^{C F \times D F}$ & $\Delta A^{\text {Total }}$ \\
\hline $\mathrm{ClCOOH} . . . \mathrm{BrCOOH}$ & 126,7 & $-207,4$ & 61,9 & 2551,7 & 262,1 & 676,5 & 3471,4 \\
$\mathrm{BrCOOH} . . . \mathrm{HCOOH}$ & 150,2 & $-344,6$ & 48,5 & 2416,8 & 193,3 & 621,3 & 3085,4 \\
$\mathrm{CH}_{3} \mathrm{COOH} . . . \mathrm{HCOOH}$ & 177,6 & $-417,9$ & 37,2 & 2361,3 & 48,7 & 487,5 & 2694,5 \\
$\mathrm{CH}_{3} \mathrm{COOH} . . . \mathrm{FCOOH}$ & 144,2 & $-322,7$ & 44,6 & 2265,9 & 212,8 & 541,5 & 2886,3 \\
$\mathrm{CH}_{3} \mathrm{COOH} . . . \mathrm{ClCOOH}$ & 132,6 & $-178,1$ & 29,2 & 2630,3 & 146,1 & 646,0 & 3406,1 \\
$\mathrm{CH}_{3} \mathrm{COOH} . . . \mathrm{BrCOOH}$ & 145,0 & $-117,4$ & 37,8 & 2744,4 & 171,6 & 722,8 & 3704,2 \\
\hline${ }^{\mathrm{a}} \Delta A=\left[\left(A_{O-H}(s)+A_{O-H}(a s)\right)_{\text {Dímero }}-\left(A_{O-H}(a)+A_{O-H}(b)\right)_{\text {Monômeros }}\right]$.
\end{tabular}

A dimerização de ácidos carboxílicos resulta na intensificação das bandas correspondentes à hidroxila (2018 a $3704 \mathrm{~km} \mathrm{~mol}^{-1}$ ), conforme mencionado anteriormente. $\mathrm{O}$ aumento da intensidade de infravermelho para os homodímeros (2018 a $3676 \mathrm{~km} \mathrm{~mol}^{-1}$ ) segue a tendência de polarizabilidade do substituinte, $\mathrm{H}<\mathrm{F}<\mathrm{Cl}<\mathrm{Br}$. Nos casos dos heterodímeros, quando se mantêm constante o monômero de ácido fórmico ou de ácido etanoico no dímero, observa-se esta mesma tendência de aumento da intensidade durante a substituição no outro monômero (2018 a 3085 e 2695 a $3704 \mathrm{~km} \mathrm{~mol}^{-1}$ para as séries contendo $\mathrm{HCOOH}$ e $\mathrm{CH}_{3} \mathrm{COOH}$, respectivamente). As bandas correspondentes aos estiramentos $\mathrm{O}-\mathrm{H}$ mais intensas são aquelas apresentadas pelos dímeros duplamente halogenados com bromo e cloro.

O modelo CCFDF/QTAIM explica esta intensificação das bandas das hidroxilas após a formação das duas ligações de hidrogênio como um resultado da variação de carga de cada átomo durante a dimerização, em conjunto com as alterações dos termos cruzados entre esta quantidade e os fluxos de carga e de dipolo atômicos, $\Delta A^{\mathrm{C}}+\Delta A^{\mathrm{C} \times \mathrm{CF}}+\Delta A^{\mathrm{C} \times \mathrm{DF}}$ (Figuras 9 e 10). Todavia, a contribuição referente ao termo cruzado entre a carga e o fluxo de carga, $\Delta A^{\mathrm{C} \times \mathrm{CF}}$, é preponderante para todos os sistemas (Figura 11), sendo capaz de explicar, isoladamente, uma parcela significativa da variação da intensidade de infravermelho dos modos de estiramento analisados $\left(\mathrm{R}^{2}=0,74\right)$, assim como observado para dímeros simples (TERRABUIO et al., 2014). Esta correlação aumenta quando o homodímero de ácido fórmico é removido da análise $\left(\mathrm{R}^{2}=0,90\right)$. Isto signfica que é a transferência de carga eletrônica, que reforça as derivadas do momento de dipolo molecular causada pelo deslocamento dos átomos carregados, que provoca o aumento da intensidade de infravermelho dos modos de estiramento $\mathrm{OH}$ durante a formação das ligações de hidrogênio. 
Figura 9 - Variação da intensidade de infravermelho dos modos de estiramento O-H durante a dimerização, $\Delta A$, em função da soma da contribuição de carga, $\Delta A^{C}$, e seu termos cruzados com fluxo de carga e fluxo de dipolo, $\Delta A^{C \times C F}$ e $\Delta A^{C \times D F}$, segundo cálculos B2PLYP/aug-cc$\mathrm{pVTZ}\left(\mathrm{em} \mathrm{km} \mathrm{mol}{ }^{-1}\right)$

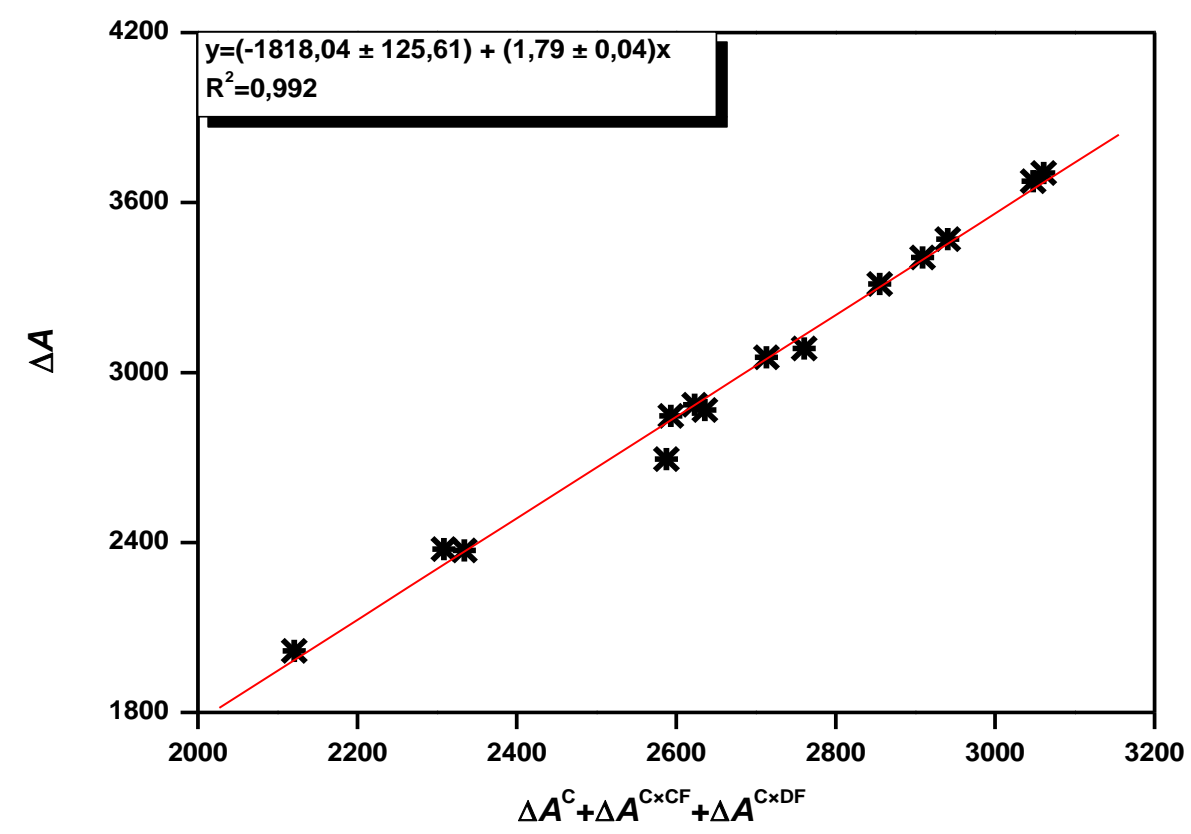

Figura 10 - Contribuições para as variações das intensidades de infravermelho totais dos modos de estiramento $\mathrm{O}-\mathrm{H}$ durante a dimerização segundo modelo CCFDF/QTAIM: (1) Cargas e seus termos cruzados com os fluxos de carga e de dipolos atômicos, $\Delta A^{\mathrm{C}}+\Delta A^{\mathrm{C} \times \mathrm{CF}}+$ $\Delta A^{\mathrm{C} \times \mathrm{DF}}$, e (2) Fluxo de carga e fluxo de dipolo e o termo cruzado entre estes dois termos, $\Delta A^{\mathrm{CF}}+\Delta A^{\mathrm{DF}}+\Delta A^{\mathrm{CF} \times \mathrm{DF}}\left(\mathrm{em} \mathrm{km} \mathrm{mol}{ }^{-1}\right)$

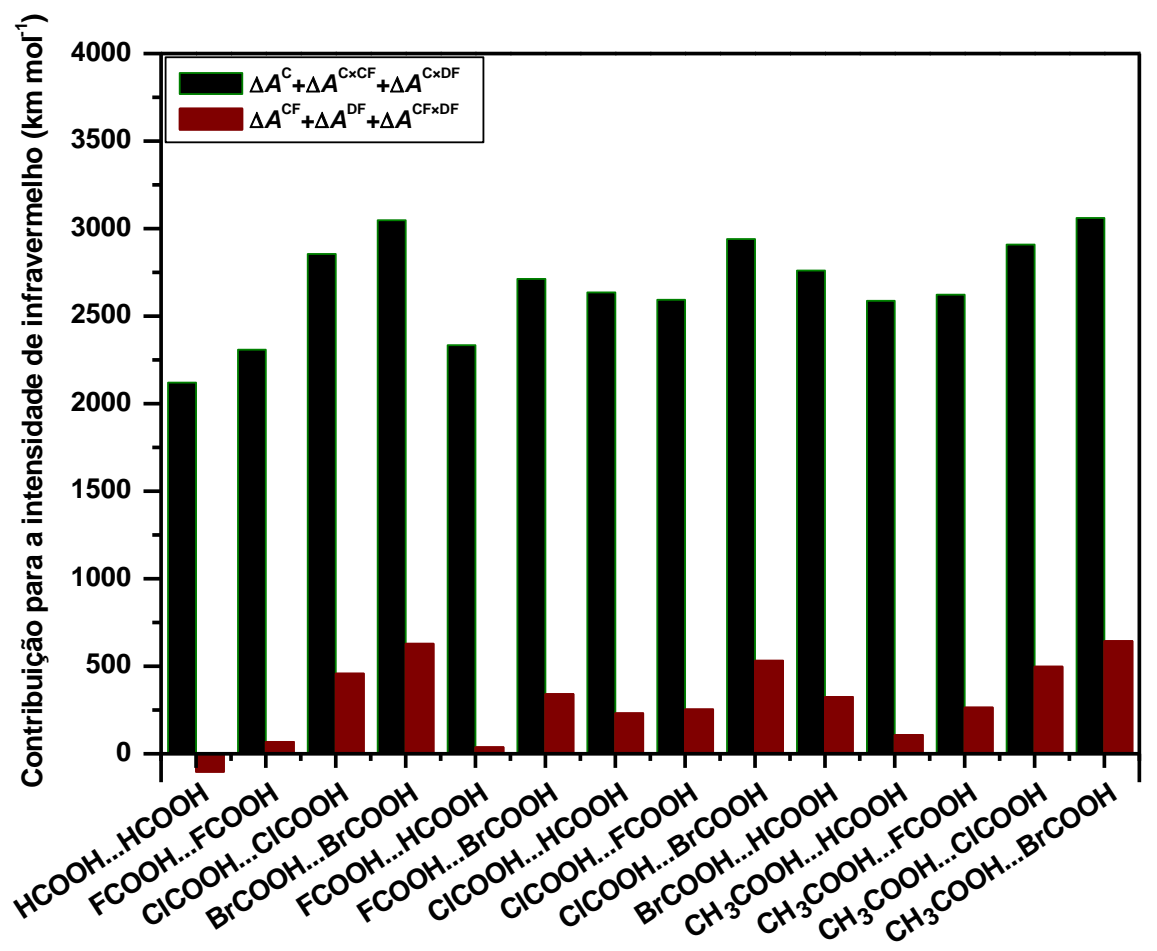


Figura 11 - Variação da intensidade de infravermelho dos modos de estiramento O-H durante a dimerização, $\Delta A$, em função da contribuição cruzada entre a carga e o fluxo de carga, $\Delta A^{\mathrm{C} \times \mathrm{CF}}$, segundo cálculos B2PLYP/aug-cc-pVTZ $\left(\mathrm{em} \mathrm{km} \mathrm{mol}^{-1}\right)$

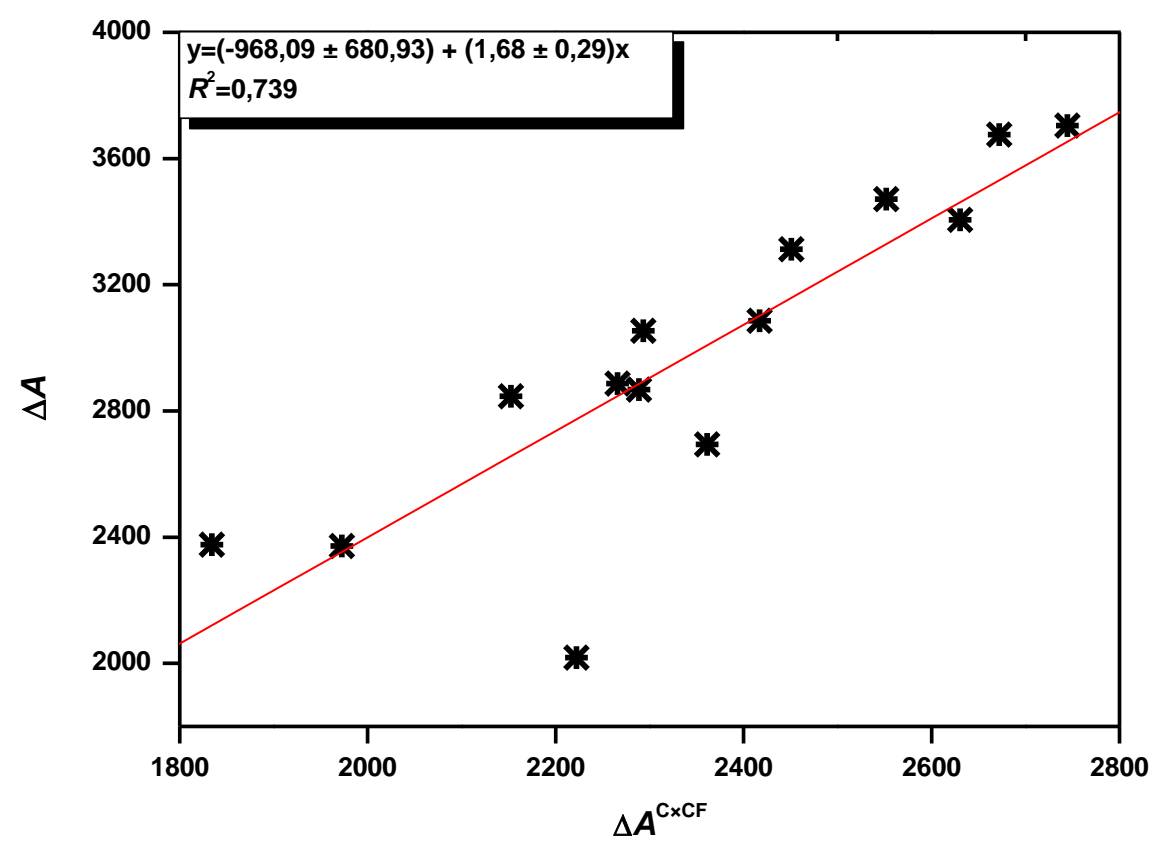

\subsection{Análise das variações de carga atômicas durante a dimerização}

A Tabela 11 mostra as variações das cargas atômicas calculadas durante a dimerização em nível B2PLYP/aug-cc-pVTZ empregando-se os formalismos QTAIM e NPA. Assim como observado para dímeros mais simples (TERRABUIO et al., 2014) e segundo ambos os modelos de carga utilizados, o átomo de hidrogênio do grupo doador de próton (hidroxila) sempre se torna mais positivo $\left(\Delta q_{Q T A I M} \approx 0,06\right.$ e $\left.\Delta q_{N P A} \approx 0,02 e\right)$ e o oxigênio da carbonila fica mais negativamente carregado $\left(\Delta q_{Q T A I M} \approx-0,03\right.$ e $\left.\Delta q_{N P A} \approx-0,07 e\right)$ durante a dimerização.

Tabela 11 - Variações nas cargas atômicas, obtidas a partir dos formalismos QTAIM e NPA, dos dímeros de ácidos carboxílicos em relação aos monômeros isolados em nível de cálculo B2PLYP/aug-cc-pVTZ (em u. a.)

\begin{tabular}{lrrr}
\hline Sistema & Átomo $^{\mathrm{a}}$ & $\Delta q(\mathrm{QTAIM})^{\mathrm{b}}$ & $\Delta q(\mathrm{NPA})^{\mathrm{b}}$ \\
\hline HCOOH...HCOOH & $\mathrm{H}$ & 0,002 & 0,010 \\
& $\mathrm{C}$ & 0,027 & 0,023 \\
& $\mathrm{O}$ & $-0,034$ & $-0,070$ \\
& $\mathrm{O}$ & $-0,060$ & 0,015 \\
& $\mathrm{H}$ & 0,064 & 0,022 \\
\hline FCOOH...FCOOH & $\mathrm{F}$ & 0,000 & 0,014 \\
& $\mathrm{C}$ & 0,035 & 0,035 \\
& $\mathrm{O}$ & $-0,033$ & $-0,079$ \\
& $\mathrm{O}$ & $-0,057$ & 0,012 \\
& $\mathrm{H}$ & 0,056 & 0,018 \\
\hline
\end{tabular}




\begin{tabular}{|c|c|c|c|}
\hline Sistema & Átomo ${ }^{\mathrm{a}}$ & 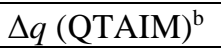 & $\Delta q(\mathrm{NPA})^{\mathrm{b}}$ \\
\hline \multirow{5}{*}{$\mathrm{ClCOOH} . . . \mathrm{ClCOOH}$} & $\mathrm{Cl}$ & 0,017 & 0,026 \\
\hline & $\mathrm{C}$ & 0,022 & 0,027 \\
\hline & $\mathrm{O}$ & $-0,038$ & $-0,084$ \\
\hline & $\mathrm{O}$ & $-0,055$ & 0,009 \\
\hline & $\mathrm{H}$ & 0,055 & 0,021 \\
\hline \multirow[t]{5}{*}{$\mathrm{BrCOOH} . . . \mathrm{BrCOOH}$} & $\mathrm{Br}$ & 0,023 & 0,030 \\
\hline & $\mathrm{C}$ & 0,015 & 0,025 \\
\hline & $\mathrm{O}$ & $-0,039$ & $-0,085$ \\
\hline & $\mathrm{O}$ & $-0,055$ & 0,008 \\
\hline & $\mathrm{H}$ & 0,055 & 0,021 \\
\hline \multirow{10}{*}{ СlCOOH...НCOOH } & $\mathrm{Cl}$ & 0,001 & 0,011 \\
\hline & $\mathrm{C}$ & 0,028 & 0,029 \\
\hline & $\mathrm{O}$ & $-0,039$ & $-0,085$ \\
\hline & $\mathrm{O}$ & $-0,063$ & 0,004 \\
\hline & $\mathrm{H}$ & 0,058 & 0,019 \\
\hline & $\mathrm{H}$ & 0,011 & 0,016 \\
\hline & $\mathrm{C}$ & 0,026 & 0,027 \\
\hline & $\mathrm{O}$ & $-0,031$ & $-0,066$ \\
\hline & $\mathrm{O}$ & $-0,051$ & 0,023 \\
\hline & $\mathrm{H}$ & 0,060 & 0,023 \\
\hline \multirow[t]{10}{*}{ СlCOOH...FCOOH } & $\mathrm{Cl}$ & 0,018 & 0,028 \\
\hline & $\mathrm{C}$ & 0,022 & 0,027 \\
\hline & $\mathrm{O}$ & $-0,039$ & $-0,087$ \\
\hline & $\mathrm{O}$ & $-0,058$ & 0,009 \\
\hline & $\mathrm{H}$ & 0,058 & 0,023 \\
\hline & $\mathrm{F}$ & 0,000 & 0,013 \\
\hline & $\mathrm{C}$ & 0,033 & 0,034 \\
\hline & $\mathrm{O}$ & $-0,032$ & $-0,076$ \\
\hline & $\mathrm{O}$ & $-0,055$ & 0,012 \\
\hline & $\mathrm{H}$ & 0,053 & 0,017 \\
\hline \multirow[t]{10}{*}{$\mathrm{ClCOOH} . . . \mathrm{BrCOOH}$} & $\mathrm{Cl}$ & 0,017 & 0,027 \\
\hline & $\mathrm{C}$ & 0,021 & 0,027 \\
\hline & $\mathrm{O}$ & $-0,037$ & $-0,083$ \\
\hline & $\mathrm{O}$ & $-0,054$ & 0,010 \\
\hline & $\mathrm{H}$ & 0,054 & 0,020 \\
\hline & $\mathrm{Br}$ & 0,022 & 0,030 \\
\hline & $\mathrm{C}$ & 0,015 & 0,025 \\
\hline & $\mathrm{O}$ & $-0,039$ & $-0,086$ \\
\hline & $\mathrm{O}$ & $-0,056$ & 0,008 \\
\hline & $\mathrm{H}$ & 0,056 & 0,022 \\
\hline \multirow[t]{10}{*}{ FCOOH...HCOOH } & $\mathrm{F}$ & $-0,002$ & 0,006 \\
\hline & $\mathrm{C}$ & 0,029 & 0,031 \\
\hline & $\mathrm{O}$ & $-0,034$ & $-0,079$ \\
\hline & $\mathrm{O}$ & $-0,063$ & 0,006 \\
\hline & $\mathrm{H}$ & 0,056 & 0,014 \\
\hline & $\mathrm{H}$ & 0,011 & 0,016 \\
\hline & $\mathrm{C}$ & 0,026 & 0,028 \\
\hline & $\mathrm{O}$ & $-0,033$ & $-0,069$ \\
\hline & $\mathrm{O}$ & $-0,054$ & 0,022 \\
\hline & $\mathrm{H}$ & 0,063 & 0,025 \\
\hline \multirow[t]{5}{*}{ FCOOH...BrCOOH } & $\mathrm{F}$ & 0,000 & 0,013 \\
\hline & $\mathrm{C}$ & 0,033 & 0,034 \\
\hline & $\mathrm{O}$ & $-0,032$ & $-0,075$ \\
\hline & $\mathrm{O}$ & $-0,054$ & 0,013 \\
\hline & $\mathrm{H}$ & 0,052 & 0,016 \\
\hline
\end{tabular}




\begin{tabular}{|c|c|c|c|}
\hline \multirow[t]{6}{*}{ Sistema } & Átomo ${ }^{\mathrm{a}}$ & 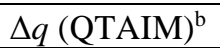 & $\Delta q(\mathrm{NPA})^{\mathrm{b}}$ \\
\hline & $\mathrm{Br}$ & 0,024 & 0,032 \\
\hline & $\mathrm{C}$ & 0,014 & 0,026 \\
\hline & $\mathrm{O}$ & $-0,041$ & $-0,090$ \\
\hline & $\mathrm{O}$ & $-0,058$ & 0,007 \\
\hline & $\mathrm{H}$ & 0,059 & 0,024 \\
\hline \multirow[t]{10}{*}{ ВrCOOH...HCOOH } & $\mathrm{Br}$ & 0,001 & 0,012 \\
\hline & $\mathrm{C}$ & 0,026 & 0,028 \\
\hline & $\mathrm{O}$ & $-0,040$ & $-0,086$ \\
\hline & $\mathrm{O}$ & $-0,064$ & 0,003 \\
\hline & $\mathrm{H}$ & 0,060 & 0,020 \\
\hline & $\mathrm{H}$ & 0,012 & 0,016 \\
\hline & $\mathrm{C}$ & 0,025 & 0,027 \\
\hline & $\mathrm{O}$ & $-0,031$ & $-0,065$ \\
\hline & $\mathrm{O}$ & $-0,050$ & 0,023 \\
\hline & $\mathrm{H}$ & 0,059 & 0,022 \\
\hline \multirow[t]{13}{*}{$\mathrm{CH}_{3} \mathrm{COOH} . . . \mathrm{HCOOH}$} & $\mathrm{C}$ & 0,000 & 0,007 \\
\hline & $\mathrm{H}$ & $-0,001$ & $-0,001$ \\
\hline & $\mathrm{H}$ & 0,004 & 0,003 \\
\hline & $\mathrm{H}$ & 0,004 & 0,003 \\
\hline & $\mathrm{C}$ & 0,028 & 0,032 \\
\hline & $\mathrm{O}$ & $-0,030$ & $-0,071$ \\
\hline & $\mathrm{O}$ & $-0,062$ & 0,011 \\
\hline & $\mathrm{H}$ & 0,063 & 0,025 \\
\hline & $\mathrm{H}$ & $-0,002$ & 0,007 \\
\hline & $\mathrm{C}$ & 0,028 & 0,021 \\
\hline & $\mathrm{O}$ & $-0,034$ & $-0,070$ \\
\hline & $\mathrm{O}$ & $-0,064$ & 0,012 \\
\hline & $\mathrm{H}$ & 0,066 & 0,022 \\
\hline \multirow[t]{13}{*}{$\mathrm{CH}_{3} \mathrm{COOH} \ldots \mathrm{FCOOH}$} & $\mathrm{C}$ & 0,002 & 0,007 \\
\hline & $\mathrm{H}$ & 0,003 & 0,001 \\
\hline & $\mathrm{H}$ & 0,008 & 0,005 \\
\hline & $\mathrm{H}$ & 0,008 & 0,005 \\
\hline & $\mathrm{C}$ & 0,023 & 0,038 \\
\hline & $\mathrm{O}$ & $-0,030$ & $-0,071$ \\
\hline & $\mathrm{O}$ & $-0,056$ & 0,018 \\
\hline & $\mathrm{H}$ & 0,062 & 0,027 \\
\hline & $\mathrm{F}$ & $-0,003$ & 0,003 \\
\hline & $\mathrm{C}$ & 0,026 & 0,029 \\
\hline & $\mathrm{O}$ & $-0,034$ & $-0,079$ \\
\hline & $\mathrm{O}$ & $-0,067$ & 0,003 \\
\hline & $\mathrm{H}$ & 0,057 & 0,013 \\
\hline \multirow[t]{13}{*}{$\mathrm{CH}_{3} \mathrm{COOH} . . . \mathrm{ClCOOH}$} & $\mathrm{C}$ & 0,002 & 0,007 \\
\hline & $\mathrm{H}$ & 0,003 & 0,001 \\
\hline & $\mathrm{H}$ & 0,008 & 0,005 \\
\hline & $\mathrm{H}$ & 0,008 & 0,005 \\
\hline & $\mathrm{C}$ & 0,022 & 0,037 \\
\hline & $\mathrm{O}$ & $-0,028$ & $-0,067$ \\
\hline & $\mathrm{O}$ & $-0,053$ & 0,019 \\
\hline & $\mathrm{H}$ & 0,059 & 0,025 \\
\hline & $\mathrm{Cl}$ & $-0,007$ & 0,003 \\
\hline & $\mathrm{C}$ & 0,032 & 0,029 \\
\hline & $\mathrm{O}$ & $-0,038$ & $-0,084$ \\
\hline & $\mathrm{O}$ & $-0,067$ & 0,001 \\
\hline & $\mathrm{H}$ & 0,060 & 0,019 \\
\hline $\mathrm{CH}_{3} \mathrm{COOH} . . . \mathrm{BrCOOH}$ & $\mathrm{C}$ & 0,002 & 0,007 \\
\hline
\end{tabular}




\begin{tabular}{rrrr}
\hline Sistema & Átomo $^{\mathrm{a}}$ & $\Delta q(\mathrm{QTAIM})^{\mathrm{b}}$ & $\Delta q(\mathrm{NPA})^{\mathrm{b}}$ \\
\hline $\mathrm{H}$ & 0,004 & 0,001 \\
$\mathrm{H}$ & 0,008 & 0,005 \\
$\mathrm{H}$ & 0,008 & 0,005 \\
$\mathrm{C}$ & 0,021 & 0,038 \\
$\mathrm{O}$ & $-0,028$ & $-0,066$ \\
$\mathrm{O}$ & $-0,052$ & 0,020 \\
$\mathrm{H}$ & 0,057 & 0,024 \\
$\mathrm{Br}$ & $-0,010$ & 0,002 \\
$\mathrm{C}$ & 0,032 & 0,030 \\
$\mathrm{O}$ & $-0,039$ & $-0,085$ \\
$\mathrm{O}$ & $-0,067$ & 0,001 \\
$\mathrm{H}$ & 0,062 & 0,019 \\
\hline
\end{tabular}

a Os átomos foram apresentados na ordem em que a estrutura do dímero foi escrita;

${ }^{\mathrm{b}} \Delta q=q$ (dímero) $-q$ (monômeros).

\section{CONCLUSÕES}

De modo geral, observa-se que a dimerização de ácidos carboxílicos resulta na redução das frequências vibracionais e no aumento das intensidades de infravermelho para os modos de estiramento das ligações dos grupamentos hidroxila $(\mathrm{O}-\mathrm{H})$. Segundo o modelo CCFDF/QTAIM, a intensificação das bandas O-H pode ser justificada pelas mudanças nas cargas, em conjunto com as alterações coordenadas de fluxo de carga e fluxo de dipolo $\left(\triangle A^{C F}\right.$ $\left.+\triangle A^{C \times C F}+\Delta A^{C F \times D F}\right)$, as quais ocorrem durante a formação de ligações de hidrogênio. Todavia, considerando-se isoladamente as contribuições para a intensidade de infravermelho, o termo cruzado entre a carga e o fluxo de carga $\left(\triangle A^{C \times C F}\right)$ é capaz de explicar a maior parte do aumento tão expressivo desta propriedade espectroscópica, o que também foi observado para dímeros mais simples (TERRABUIO et al., 2014). Inclusive, Terrabuio et al. (2014) lançaram a hipótese de esta ser uma característica geral de sistemas com ligações de hidrogênio.

Em relação às propriedades topológicas nos pontos críticos das ligações de hidrogênio, observa-se que (1) a densidade eletrônica é maior quando o oxigênio da carbonila do ácido etanoico interage com o hidrogênio da hidroxila de monômeros halogenados, (2) o Laplaciano é maior que zero e (3) todos os valores da densidade de energia total são negativos para os sistemas estudados, indicando um predomínio da energia potencial sobre a energia cinética nas interações O...H. Estes dados são condizentes com ligações de hidrogênio de força média. Além disto, a dimerização de ácidos carboxílicos é acompanhada por uma diminuição da densidade eletrônica e um aumento do Laplaciano da densidade nos BCPs das ligações O-H, resultando no enfraquecimento e um concomitante aumento do caráter iônico destas ligações. 
A partição energética de Átomos Quânticos Interagentes também mostra que as ligações de hidrogênio de ácidos carboxílicos são de força média e que a energia de interação para as ligações $\mathrm{O}$...H e a energia de dimerização se correlacionam de modo linear com o Laplaciano da densidade eletrônica nos BCPs e RCPs, respectivamente. Nestes casos, observa-se uma separação dos dímeros em dois grupos, em que um deles sempre apresenta ácido fórmico ou o ácido acético como um dos monômeros enquanto o outro é formado por dímeros apenas de monômeros halogenados. Por fim, durante a dimerização, o hidrogênio da hidroxila perde um pouco de carga eletrônica, enquanto o oxigênio da carbonila fica mais negativamente carregado.

\section{PERSPECTIVAS}

Investigar os efeitos cooperativos das ligações de hidrogênio para as intensidades de infravermelho dos dímeros de ácidos carboxílicos. Para isso, seria necessário a realização de cálculos desta propriedade em diferentes arranjos estruturais dos dímeros, avaliando-se a formação de uma ligação de hidrogênio por vez, de modo a comparar os resultados obtidos com aqueles apresentados neste capítulo.

\section{REFERÊNCIAS}

ARUNAN, E., DESIRAJU, G. R., KLEIN, R. A., SADLEJ, J., SCHEINER, S., ALKORTA, I., CLARY, D. C., CRABTREE, R. H., DANNENBERG, J. J., HOBZA, P., KJAERGAARD, H. G., LEGON, A. C.; MENNUCCI, B. e NESBITT, D. J. Definition of the hydrogen bond. Pure Applied Chemistry, v. 83, p. 1637-1641, 2011.

BADER, R. F. W. Atoms in Molecules: A Quantum Theory, Clarendon Press: Oxford, 1990. Capítulos 1 e 7, 13-52 e 248-351 p.

BLANCO, M. A.; PENDÁS, A. M. e FRANCISCO, E. Interacting quantum atoms: a correlated energy decomposition scheme based on the quantum theory of atoms in molecules. Journal of Chemical Theory and Computation, v. 1, p. 1096-1109, 2005.

BOYS, S. F. e BERNARDI, F. The calculation of small molecular interactions by the differences of separate total energies. Some procedures with reduced errors. Molecular Physics, v. 19, p. 553-566, 1970.

CREMER, D e KRAKA, E: in Conceptual Quantum Chemistry, Models and Applications, Z. B. Maksić, Ed., Croatica Chemica Acta, v. 57, p. 1259-1281, 1984.

KOCH, U. e POPELIER, P. L. A. Characterization of C-H-O hydrogen bonds on the basis of the charge density. The Journal of Chemical Physics, v. 99, p. 9747-9754, 1995. 
Da SILVA, N. A e HAIDUKE, R. L. A. Infrared intensity analysis of hydroxyl stretching modes in carboxylic acid dimers by means of the Charge-Charge flux-Dipole flux model. Journal of Computational Chemistry, v. 40, p. 2482-2490, 2019.

DESIRAJU, G. R. e STEINER, T. The weak hydrogen bond: in structural chemistry e biology; Oxford University Press, Inc.: New York, 1999, Capítulo 1, 1-28 p.

DUNNING JR, T. H. Gaussian basis sets for use in correlated molecular calculations. I. The atoms boron through neon and hydrogen. The Journal of Chemical Physics, v. 90, p. 10071023, 1989.

FRISCH, M. J.; TRUCKS G. W.; SCHLEGEL, H. B.; SCUSERIA, G. E.; ROBB, M. A.; CHEESEMAN, J. R.; SCALMANI, G.; BARONE, V.; MENNUCCI, B.; PETERSSON, G. A.; NAKATSUJI, H.; CARICATO, M.; LI, X.; HRATCHIAN, H. P.; IZMAYLOV, A. F.; BLOINO, J.; ZHENG, G.; SONNENBERG, J. L.; HADA, M.; EHARA, M.; TOYOTA, K.; FUKUDA, R.; HASEGAWA, J.; ISHIDA, M.; NAKAJIMA, T.; HONDA, Y.; KITAO, O.; NAKAI, H.; VREVEN, T.; MONTGOMERY JR., J. A.; PERALTA, J. E.; OGLIARO, F.; BEARPARK, M.; HEYD, J. J.; BROTHERS, E.; KUDIN, K. N.; STAROVEROV, V. N.; KEITH, T.; KOBAYASHI, R.; NORMAND, J.; RAGHAVACHARI, K.; RENDELL, A.; BURANT, J. C.; IYENGAR, S. S.; TOMASI, J.; COSSI, M.; REGA, N.; MILLAM, J. M.; KLENE, M.; KNOX, J. E.; CROSS, J. B.; BAKKEN, V.; ADAMO, C.; JARAMILLO, J.; GOMPERTS, R.; STRATMANN, R. E.; YAZYEV, O.; AUSTIN, A. J.; CAMMI, R.; POMELLI, C.; OCHTERSKI, J. W.; MARTIN, R. L.; MOROKUMA, K.; ZAKRZEWSKI, V. G.; VOTH, G. A.; SALVADOR, P.; DANNENBERG, J. J.; DAPPRICH, S.; DANIELS, A. D.; FARKAS, O.; FORESMAN, J. B.; ORTIZ, J. V.; CIOSLOWSKI, J. e FOX, D. J. Gaussian 09, Revision D.01, Gaussian Inc., Wallingford, CT, 2013.

GALIMBERTI, D.; MILANI, A. e CASTIGLIONI, C. Infrared intensities and charge mobility in hydrogen bonded complexes. The Journal of Chemical Physics, v. 139, p. 115$164,2013$.

GORA, R. W., GRABOWSKI, S. J. e LESZCZYNSKI, J. Dimers of formic acid, acetic acid, formamide and Pyrrole-2-carboxylic acid: an ab initio study. The Journal of Physical Chemistry A, v. 109, p. 6397-6405, 2005.

GRABOWSKI, S. J. In The Quantum Theory of Atoms in Molecules: From Solid State to DNA e Drug Design; Matta, C. F. e Boyd R. J., Eds.; Wiley-VCH: Weinheim, 2007; Capítulo 17, 453-469 p.

GRABOWSKI, S. J. What is the covalency of hydrogen bonding? Chemical Reviews, v. 111, p. $-2597-2625,2011$.

GRABOWSKI. S. J. Ab initio calculations on conventional and unconventional hydrogen bonds-study of the hydrogen bond strength. The Journal of Chemical Physics A, v. 105, p. 10739-10746, 2001.

GRABOWSKI, S. J. Properties of a ring critical point as measures of intramolecular H-bond strength. Monatshefte für Chemie. v. 133, p. 1373-1380, 2002.

GRIMME, S. Semiempirical hybrid density functional with perturbative second-order 
correlation. The Journal of Chemical Physics, v. 124, p. 1-16, 2006.

GUEVARA-VELA, J. M., ROMERO-MONTALVO, E., COSTALES, A., PENDÁS, A. M. e ROCHA-RINZA, T. The nature of ressonance-assisted hydrogen bonds: a quantum chemical topology perspective. Physical Chemistry Chemical Physics, v. 38, p. 26383-26390, 2016.

HAIDUKE, R. L. A. e BRUNS, R. E. An atomic Charge-Charge Flux-Dipole Flux Atom-inMolecule decomposition for molecular dipole-moment derivatives and infrared fundamental intensities. The Journal of Physical Chemistry A, v. 109, p. 2680-2688, 2005.

HAURIE M. e NOVAK A. Spectres de vibration des molecules $\mathrm{CH}_{3} \mathrm{COOH}, \mathrm{CH}_{3} \mathrm{COOD}$, $\mathrm{CD}_{3} \mathrm{COOH}$ et $\mathrm{CD}_{3} \mathrm{COOD}-\mathrm{III}$. Spectres infrarouges des cristaux. The Journal of Chemical Physics, v. 62, p. 137-145, 1965.

HAYNES, W. M. Ed. CRC Handbook of Chemistry e Physics; 96th ed. CRC Press, Taylor e Francis: Boca Raton, FL, 2016.

ISAACS, E. D.; SHUKLA A.; PLATZMAN, P. M.; HAMANN, D. R.; Barbiellini, B. e C. A. Tulk. Covalency of the hydrogen bond in ice: a direct X-ray measurement. Physical Review Letters, v. 82, p. 600-603, 1999.

JEFFREY, G. A. e SAENGER, W. Hydrogen Bonding in Biological Structures; Springer: Berlin, 1991, Capítulo 2, 15-48 p.

KEITH, T. A. AIMAll (version 16.10.31), TK Gristmill Software, Overle Park. KS 2016. Available at: aim.tkgristmill.com (acessado em 10 de março de 2016).

KENDALL, R. A.; DUNNING JR., T.H.; HARRISON, R.J. Electron affinities of the first-row atoms revisited. Systematic basis sets and wave functions. The Journal of Chemical Physics, v. 96, p. 6796, 1992.

KRISHER, L. C. e SAEGEBARTH. Microwave spectrum of acetic Acid, $\mathrm{CH}_{3} \mathrm{COOH}$ and $\mathrm{CD}_{3} \mathrm{COOH}$. The Journal of Chemical Physics, v. 54, p. 4553-4558, 1971.

MATTA, C. F. e BOYD, R. J. The Quantum Theory of Atoms in Molecules From Solid State to DNA and Drug Design, Weinheim: Wiley-Vch, 2007. 1-21 p.

MO. Y. Can QTAIM topological parameters be a measure of hydrogen bonding strength? The Journal of Chemical Physics A, v. 116, p. 5240-5246, 2012.

PENDÁS, A. M.; BLANCO, M. A. e FRANCISCO, E. The nature of the hydrogen bond: a synthesis from the interacting quantum atoms picture. The Journal of Chemical Physics, v. 125 , p. 184-112, 2006.

REED, A. E.; WEINSTOCK, R. B. e WEINHOLD, F. Natural population analysis. The Journal of Chemical Physics, v. 83, p. 735-746, 1985.

ROZAS, I.; ALKORTA, I. e ELGUERO, J. Behavior of ylides containing n, o, and c atoms as hydrogen bond acceptors. Journal of the American Chemical Society, v. 122, p. 1115411161, 2000. 
RUSU, V. H., RAMOS, M. N. e LONGO, R. L. CCFOM and CCFDF models to interpret infrared intensities: a comparative study. Journal of Molecular Structure, v. 993, p. 86-90, 2011.

RYBARCZYK-PIREK, A. J.; GRABOWSKI, S. J.; MAŁECKA, M. e NAWROTMODRANKA, J. Crystal and molecular structures of new chromone derivatives as empirical evidence of intramolecular proton transfer reaction; ab initio studies on intramolecular $\mathrm{H}$ bonds in enaminones. The Journal of Chemical Physics, v. 106, 11956-11962, 2002.

SCHAFTENAAR, G. e NOORDIK J. H. Molden: a pre- and post-processing program for molecular and electronic structures, Journal Computational - Aided Molecular Design, v. 14, p. 123-134, 2000.

SHAHI, A. e ARUNAN, E. Hydrogen bonding, halogen bonding and lithium bonding: in atoms in molecules and natural bond orbital perspective towards conservation of total bond order, inter- and intra-molecular bonding. Physical Chemistry Chemical Physics, v. 16, p. 22935-22952, 2014.

SILVA, A. F.; RICHTER, W. E.; TERRABUIO, L. A.; HAIDUKE, R. L. A. e BRUNS, R. E. Quantum theory of atoms in molecules/charge-charge flux-dipole flux models for fundamental vibrational intensity changes on $\mathrm{H}$-bond formation of water and hydrogen fluoride. The Journal of Chemical Physics A, v. 140, 084306, 2014.

SILVA, A. F; RICHTER, W. E. e BRUNS, R. E. Quantum Theory of Atoms in Molecules/Charge-Charge Flux-Dipole Flux interpretation of fundamental vibrational intensity enhancements on H-bond formation of water trimer. Chemical Physics Letters, v. 610-611, p. 14-18, 2014.

TERRABUIO, L. A.; RICHTER, W. E., SILVA, A. F.; BRUNS, R. E. e HAIDUKE, R. L. A. An atom in molecules study of infrared intensity enhancements in fundamental donor stretching bands in hydrogen bond formation. Physical Chemistry Chemical Physics, v. 16, p. 24920-24928, 2014.

WILSON, A. K.; WOON, D. E.; PETERSON, K. A. e DUNNING JR, T. H. Gaussian basis sets for use in correlated molecular calculations. IX. The atoms gallium through krypton. Journal of Chemical Physics, v. 110, 7667-7676, 1999.

WOON, D. E. e DUNNING Jr., T. H. Gaussian-basis sets for use in correlated molecular calculations. 3. The atoms aluminum through argon. The Journal of Chemical Physics, v. 98, p. 1358-1371, 1993.

YOKOYAMA, I.; MIWA, Y. e MACHIDA, K. Extended molecular mechanics calculations of thermodynamic quantities, structures, vibrational frequencies, and infrared absorption intensities of formic acid monomer and dimer. Journal of the American Chemical Society, v. 113, p. 6458-6464, 1991. 
Capítulo 3

Análise de carga, fluxo de carga e fluxo de dipolo para as intensidades de infravermelho dos modos de estiramento $\mathrm{CO}$ em carbonilas metálicas 


\section{INTRODUÇÃO}

O carbonil (CO) é um dos ligantes mais comuns na química dos organometálicos, de modo que sistemas M-CO (em que M é um metal de transição) são exemplos clássicos para se investigar a natureza deste tipo de ligação (HUHEEY, KEITER, E. A., KEITER, R. L., 1993; WRIGTON, 1974). O grupo CO tipicamente estabiliza estados de oxidação mais baixos do metal gerando complexos eletricamente neutros. As carbonilas metálicas, como estes compostos são denominados, podem ser empregadas em diversas aplicações, como em catálises homogêneas e heterogêneas (DAVIDE et al., 2010). De modo geral, estes complexos podem ser sintetizados a partir do próprio $\mathrm{CO}$, por exemplo, a formação do pentacarbonilferro $(0)$

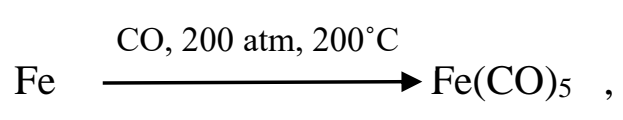

no entanto, a maioria destas reações diretas requerem pressões e temperaturas elevadas (CRABTREE, 2005). Outra metodologia para se sintetizar complexos M-CO, denominada carbonilação redutiva, é por meio da redução de sais ou complexos metálicos na presença do ligante (Ibidem):

$$
\mathrm{NiSO}_{4}+\mathrm{CO}+\mathrm{S}_{2} \mathrm{O}_{4}{ }^{2-} \rightarrow \mathrm{Ni}(\mathrm{CO})_{4}
$$

As carbonilas metálicas simples possuem formas bem definidas e simétricas, nas quais os ligantes CO ocupam as posições mais distantes. Por exemplo, as hexacarbonilas do grupo 6, como o hecarbonilcromo $(0)\left(\mathrm{Cr}(\mathrm{CO})_{6}\right)$ e suas espécies isoeletrônicas $\left(\left[\mathrm{Mn}(\mathrm{CO})_{6}\right]^{+}\right.$, $\left[\mathrm{Fe}(\mathrm{CO})_{6}\right]^{2+}$ e $\left.\left[\mathrm{Co}(\mathrm{CO})_{6}\right]^{3+}\right)$ são octaédricas, o pentacarbonilferro(0) $\left(\mathrm{Fe}(\mathrm{CO})_{5}\right)$ possui geometria bipiramidal trigonal e o tetracarbonilníquel $(0)\left(\mathrm{Ni}(\mathrm{CO})_{4}\right)$ é tetraédrico. A ligação entre o metal e o ligante nestes sistemas é descrita classicamente pelo modelo Dewar-ChattDuncanson (1951 e 1953) de doação e retrodoação $\pi$ (HOUSECROFT e SHARPE, 2008). Assim, a ligação entre o metal e o ligante baseia-se em uma interação sinérgica entre a doação do tipo $\sigma$ e a retrodoação do tipo $\pi$ (REED e DUNCAN, 2010). A ampla gama de complexos carbonílicos de metais de transição $\left(\mathrm{M}(\mathrm{CO})_{\mathrm{n}}\right.$, em que $\mathrm{M}=\mathrm{Cu}, \mathrm{Fe}, \mathrm{Mn}, \mathrm{Ni}, \mathrm{Cr}$ e $\mathrm{Co}$, dentre outros) pode ser atribuída em parte à interação $\sigma$ do orbital molecular ocupado de mais alta energia (HOMO) do CO com os orbitais não preenchidos do centro metálico (LUMO). 
A Figura 12 mostra a sobreposição entre o orbital ocupado $d_{\pi}$ do metal e o orbital $\pi^{*}$ do CO para formar a ligação M-CO. Apesar de o orbital $\pi^{*}(\mathrm{CO})$ ser antiligante apenas em relação ao carbono e oxigênio, ele ainda pode ser ligante com respeito ao metal e ao carbono (CRABTREE, 2005). Os orbitais antiligantes $\pi^{*}$ dos grupos CO possuem energia mais alta, porém são capazes de estabilizar os orbitais $d_{\pi}$, resultando em um aumento do desdobramento do campo ligante, como mostrado na Figura 12, o que explica o fato de o CO ser um ligante de campo forte, embora seja eletricamente neutro (Ibidem). Além disto, quanto mais eficiente for o processo de retrodoação, mais estáveis serão os complexos formados, pois uma parte da densidade eletrônica do metal retornará para os ligantes. Estudos teóricos e espectroscópicos mostram que, para o $\mathrm{CO}$, a retrodoação $\pi$ é frequentemente comparável ou maior do que a doação de densidade eletrônica do ligante para o metal na ligação $\sigma$ (GOLDMAN e KROGHJESPERSEN, 1996).

Figura 12 - Sobreposição entre os orbitais HOMO e LUMO do metal e do ligante e o efeito da ligação $\pi$ sobre o desdobramento do campo ligante

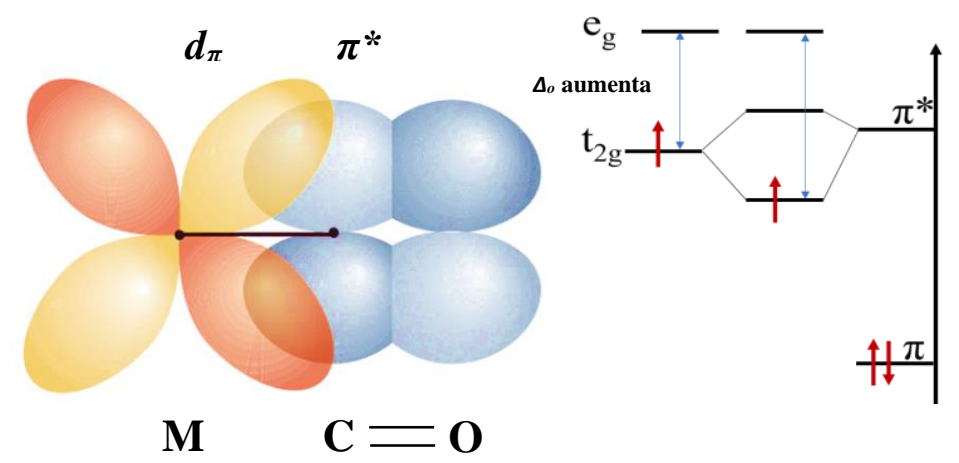

Fonte: Adaptado de CRABTREE, R. H. The organometallic chemistry of the transition metals. $4^{\mathrm{a}}$ ed. New Jersey, US: John Wiley \& Sons, 2005.

O efeito sinérgico de doação-retrodoação de carga eletrônica entre o metal e o ligante aceptor $\pi$ acarreta alterações substanciais nas frequências vibracionais e nas intensidades de infravermelho quando o ligante carbonil está coordenado a centros metálicos distintos (CORTÉS-GUZMÁN e BADER, 2005). A espectroscopia vibracional é um dos métodos mais relevantes para se caracterizar carbonilas metálicas. A frequência vibracional do grupo CO é usada, por exemplo, para examinar a natureza da ligação entre o metal e o ligante, visto que o deslocamento desta banda depende da estrutura eletrônica do complexo e correlaciona-se com a habilidade de doação de densidade eletrônica do metal (RICKS et al., 2009). 
De modo geral, a frequência do estiramento carbonil é menor que aquela apresentada pelo ligante livre devido a uma diminuição na densidade eletrônica ligante e um aumento na densidade eletrônica antiligante nos orbitais $\pi^{*}$ e isto é consistente com o enfraquecimento da ligação CO (REED e DUNCAN, 2010). Consequentemente, carbonilas metálicas que apresentam este tipo de variação da frequência, que tende a ser maior quanto mais eficiente for a retrodoação, apresentam a banda correspondente ao estiramento $\mathrm{CO}$ deslocada para o vermelho (REED e DUNCAN, 2010; GOLDMAN e KROGH-JESPERSEN, 1996). Como este fenômeno é, geralmente, observado para a maioria destes complexos, eles são denominados de carbonilas clássicas. Em raras situações, todavia, observa-se o deslocamento para o azul, isto é, a frequência vibracional $\mathrm{CO}$ do sistema $\mathrm{M}-\mathrm{CO}$ é maior do que a apresentada pelo ligante livre e, nestes casos, as carbonilas apresentam um comportamento não-clássico (GOLDMAN e KROGH-JESPERSEN, 1996). Em suma, o que determinará se a carbonila apresentará um comportamento clássico ou não clássico é a relevância ou não da retrodoação $\pi$ para o composto (LUPINETTI, FRENCKING e STRAUSS, 1998)

A frequência do estiramento $\mathrm{CO}$ constitui um bom indicador da densidade eletrônica do metal e, frequentemente, está correlacionada com outras maneiras de se estimar o caráter nucleofílico, como a facilidade de se remover um elétron (CRABTREE, 2005). Além disto, as carbonilas metálicas, geralmente, apresentam intensidades de infravermelho elevadas, constituindo-se outra família representativa de moléculas para a interpretação das intensidades de infravermelho segundo a partição Carga - Fluxo de Carga - Fluxo de dipolo em função dos multipolos da Teoria Quântica de Átomos e Moléculas (CCFDF/QTAIM) (HAIDUKE e BRUNS, 2005; BADER, 1990). A comparação de complexos isoeletrônicos ou ligantes pode ser útil para se analisar propriedades e apontar os contrastes entre estes sistemas. Por isso, além das carbonilas metálicas, o complexo $\left[\mathrm{Zn}(\mathrm{CN})_{4}\right]^{2-}$, contendo o ligante ciano $\left(\mathrm{CN}^{-}\right)$, análogo iônico do $\mathrm{CO}$, foi incluído neste trabalho. Em geral, os efeitos de polarização apresentam grande relevância na determinação da reatividade de ligantes insaturados e os mesmos efeitos observados para o $\mathrm{CO}$ podem ser estendidos para outros grupos (CRABTREE, 2005).

A investigação da anticorrelação entre os fluxos de carga e de dipolo atômicos de compostos organometálicos pode contribuir, por exemplo, para o estabelecimento de novas propostas sobre as ligações químicas presentes nestes compostos de coordenação, visto que a carga do metal de transição pode provocar importantes alterações na polarização e na distribuição da densidade eletrônica dos orbitais do CO (GOLDMAN e KROGH- 
JESPERSEN, 1996; LUPINETTI, FRENCKING, e STRAUSS, 1998). Estas mudanças podem tornar o carbono e o oxigênio mais sensíveis aos ataques nucleofílicos e eletrofílicos, respectivamente. Além do estudo das propriedades espectroscópicas, nós analisamos quantidades topológicas da densidade eletrônica nos pontos críticos das ligações entre o metal e o ligante, o carbono e o oxigênio e o carbono e nitrogênio, com o fim de caracterizar e compreender melhor estes tipos de interações. O caráter iônico e covalente destas ligações também foi investigado por meio da partição de Átomos Quânticos Interagentes (PENDÁS, BLANCO e FRANCISCO, 2005 e 2006).

\section{OBJETIVOS}

2.1 Investigar o efeito da complexação sobre as propriedades espectroscópicas das carbonilas metálicas segundo o modelo de Carga - Fluxo de Carga - Fluxo de Dipolo em termos dos multipolos da Teoria Quântica de Átomos em Moléculas (CCFDF/QTAIM);

2.2 Analisar as propriedades topológicas QTAIM nos pontos críticos das ligações metalligante, carbono-oxigênio e carbono-nitrogênio;

2.3 Avaliar o caráter iônico e covalente das ligações presentes nos complexos de coordenação de acordo com a Teoria de Átomos Quânticos Interagentes (IQA).

\section{METODOLOGIA}

Os cálculos de estrutura eletrônica das carbonilas metálicas foram realizados no programa Gaussian 09 (FRISCH et al., 2013) utilizando-se o funcional duplo híbrido B2PLYP (GRIMME, 2006) com o conjunto de funções de base aug-cc-pVTZ (DUNNING JR., 1989; KENDALL, DUNNING JR. e HARRISON, 1992; WILSON et al., 1999; WOON e DUNNING, 1993). Inicialmente, as geometrias de equilíbrio foram determinadas. $\mathrm{Na}$ sequência, as propriedades espectroscópicas (frequências vibracionais e intensidades de infravermelho) e topológicas (densidade eletrônica, Laplaciano da densidade eletrônica, densidades de energia cinética e potencial nos pontos críticos de ligação, BCPs) foram obtidas a partir das estruturas otimizadas destes compostos, as quais estão apresentadas na Figura 13. As propriedades topológicas mencionadas, assim como as cargas atômicas, foram obtidas 
utilizando-se o programa AIMAll (KEITH, 2013) por meio das densidades eletrônicas previamente calculadas

Figura 13 - Geometrias de equilíbrio dos complexos de coordenação estudados plotadas com o software Molden (version 5.3) (SCHAFTENAAR e NOORDIK, 2000)
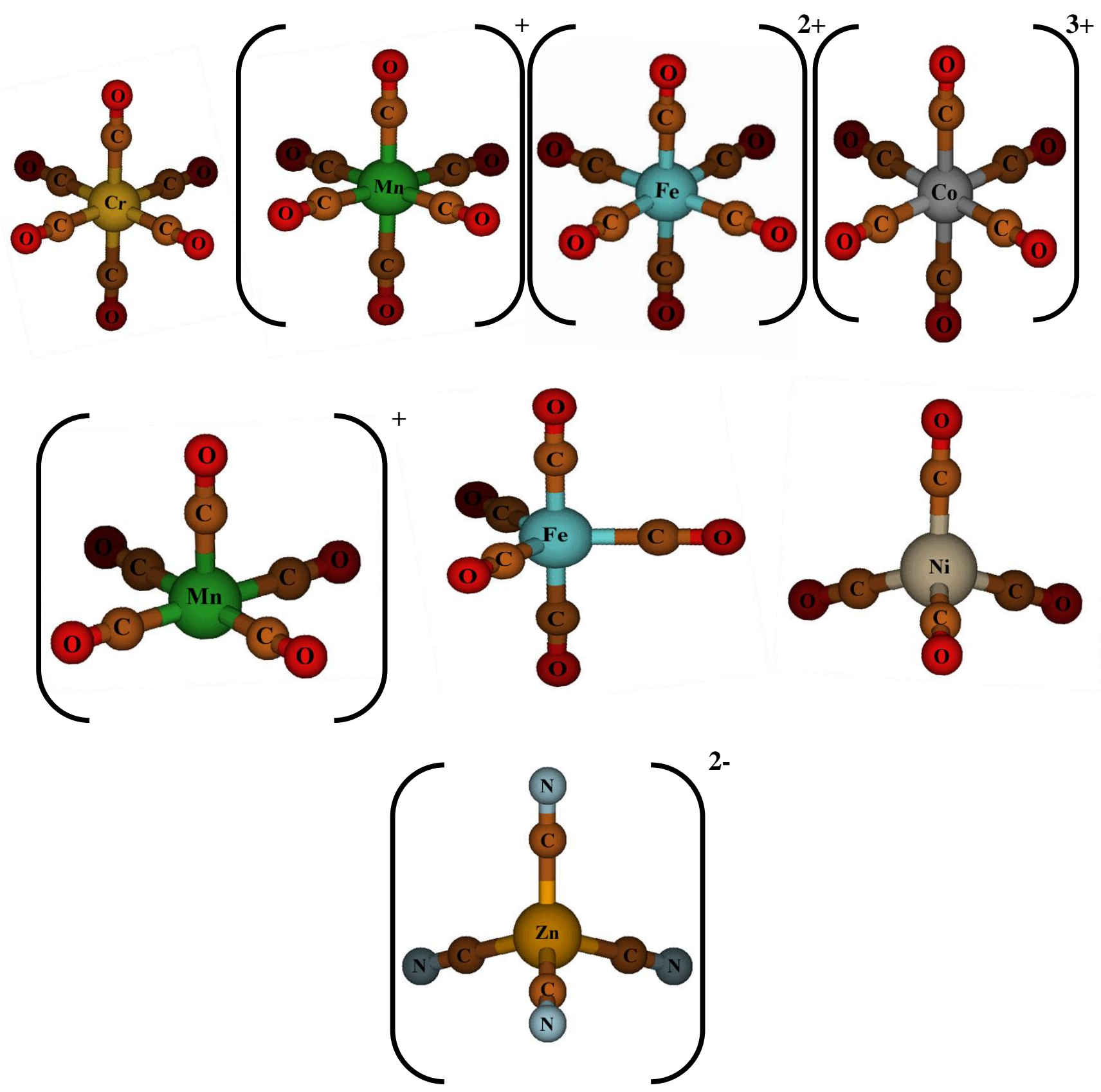

Além disso, a análise das contribuições de carga, fluxo de carga e fluxo de dipolo para as intensidades de infravermelho dos modos de estiramento $\mathrm{CO}$ segundo o modelo CCFDF/QTAIM foi realizada de acordo com o procedimento descrito para os dímeros de ácidos carboxílicos no capítulo 2. Por fim, avaliou-se o caráter iônico e covalente das ligações 
M-C, C-O e C-N por meio do formalismo de Átomos Quânticos Interagentes (IQA) (BLANCO, PENDÁS e FRANCISCO, 2005).

\section{RESULTADOS E DISCUSSÕES}

\subsection{Parâmetros geométricos}

A Tabela 12 mostra as distâncias de ligação $\mathrm{MC}, \mathrm{CO}$ e $\mathrm{CN}$ dos complexos de coordenação $\mathrm{Ni}(\mathrm{CO})_{4}, \quad\left[\mathrm{Mn}(\mathrm{CO})_{5}\right]^{+}, \quad \mathrm{Fe}(\mathrm{CO})_{5}, \quad\left[\mathrm{Co}(\mathrm{CO})_{6}\right]^{3+}, \quad\left[\mathrm{Fe}(\mathrm{CO})_{6}\right]^{2+}, \quad\left[\mathrm{Mn}(\mathrm{CO})_{6}\right]^{+}$, $\mathrm{Cr}(\mathrm{CO})_{6}$ e $\left[\mathrm{Zn}(\mathrm{CN})_{4}\right]^{2-}$. Os comprimentos de ligação apresentam boa concordância com os dados experimentais disponíveis. O maior desvio encontrado, 0,04 Å, refere-se às distâncias $\mathrm{Cr}-\mathrm{C}$ e $\mathrm{C}-\mathrm{O}$ do $\mathrm{Cr}(\mathrm{CO})_{6}$. A maioria dos complexos carbonílicos apresentam um alongamento da ligação $\mathrm{CO}$ em relação ao ligante livre, exceto para os sistemas $\left[\mathrm{Co}(\mathrm{CO})_{6}\right]^{3+}$ e $\left[\mathrm{Fe}(\mathrm{CO})_{6}\right]^{2+}$. Já para o complexo com o ligante cianeto, $\left[\mathrm{Zn}(\mathrm{CN})_{4}\right]^{2-}$, a distância de ligação $\mathrm{CN}$ diminui no sistema.

Tabela 12 - Distâncias de ligação para os ligantes $\left(\mathrm{CO}\right.$ e $\left.\mathrm{CN}^{-}\right)$livres e nos complexos metálicos, obtidas em nível de cálculo B2PLYP/aug-cc-pVTZ, bem como os dados experimentais disponíveis (em $\AA$ )

\begin{tabular}{|c|c|c|c|}
\hline Sistema & Distância & B2PLYP/aug-cc-pVTZ & Exp. \\
\hline $\mathrm{CO}$ & $\mathrm{r}(\mathrm{CO})$ & 1,131 & $1,128^{\mathrm{a}}$ \\
\hline $\mathrm{CN}^{-}$ & $\mathrm{r}(\mathrm{CN})$ & 1,179 & \\
\hline \multirow[t]{2}{*}{$\mathrm{Ni}(\mathrm{CO})_{4}$} & $\mathrm{r}(\mathrm{NiC})$ & 1,838 & $1,839^{b}$ \\
\hline & $\mathrm{r}(\mathrm{CO})$ & 1,143 & $1,121^{\mathrm{b}}$ \\
\hline \multirow[t]{3}{*}[\mathrm{Mn}(\mathrm{CO})_{5}]{$^{+}$} & $\mathrm{r}(\mathrm{MnC})_{\text {Axial }}$ & 1,890 & \\
\hline & $\mathrm{r}(\mathrm{MnC})_{\text {Equatorial }}$ & 1,789 & \\
\hline & $\mathrm{r}(\mathrm{CO})$ & 1,133 & \\
\hline \multirow[t]{2}{*}{$\mathrm{Fe}(\mathrm{CO})_{5}$} & $\mathrm{r}(\mathrm{FeC})$ & 1,803 & $1,821^{b^{\prime}}$ \\
\hline & $\mathrm{r}(\mathrm{CO})$ & 1,149 & $1,153^{\mathrm{b}}$ \\
\hline \multirow[t]{2}{*}[\mathrm{Co}(\mathrm{CO})_{6}]{$^{3+}$} & $\mathrm{r}(\mathrm{CoC})$ & 1,928 & \\
\hline & $\mathrm{r}(\mathrm{CO})$ & 1,118 & \\
\hline \multirow[t]{2}{*}[\mathrm{Fe}(\mathrm{CO})_{6}]{$^{2+}$} & $\mathrm{r}(\mathrm{FeC})$ & 1,881 & $1,911^{\mathrm{c}}$ \\
\hline & $\mathrm{r}(\mathrm{CO})$ & 1,124 & $1,104^{\mathrm{c}}$ \\
\hline \multirow[t]{2}{*}[\mathrm{Mn}(\mathrm{CO})_{6}]{$^{+}$} & $\mathrm{r}(\mathrm{MnC})$ & 1,876 & $1,905^{\mathrm{d}}$ \\
\hline & $\mathrm{r}(\mathrm{CO})$ & 1,134 & $1,123^{\mathrm{d}}$ \\
\hline \multirow[t]{2}{*}{$\mathrm{Cr}(\mathrm{CO})_{6}$} & $\mathrm{r}(\mathrm{CrC})$ & 1,915 & $1,911^{\mathrm{e}}$ \\
\hline & $\mathrm{r}(\mathrm{CO})$ & 1,147 & $1,143^{\mathrm{e}}$ \\
\hline
\end{tabular}




\begin{tabular}{llrr}
\hline Sistema & Distância & B2PLYP/aug-cc-pVTZ & Exp. \\
\hline$\left[\mathrm{Zn}(\mathrm{CN})_{4}\right]^{2-}$ & $\mathrm{r}(\mathrm{ZnC})$ & 2,073 & \\
& $\mathrm{r}(\mathrm{CN})$ & 1,169 & \\
\hline
\end{tabular}

${ }^{a}$ NATIONAL INSTITUTE OF STANDARDS E TECHNOLOGY. Chemistry webbook. U. S. Secretary of Commerce: the standard reference data act. 1968. Disponível em: <http//www.nist.gov/index.html>. Acesso em: 05 dez. 2017.

b (HAYNES, 2016); b' Valores médios das distâncias axiais e equatoriais de ligação.

c (BERNHARDT et al., 1999).

d (GEIER et al., 2007).

e (FARRUGIA e EVANS, 2005).

Analisando-se a série isoeletrônica $\mathrm{Cr}(\mathrm{CO})_{6},\left[\mathrm{Mn}(\mathrm{CO})_{6}\right]^{+},\left[\mathrm{Fe}(\mathrm{CO})_{6}\right]^{2+}$ e $\left[\mathrm{Co}(\mathrm{CO})_{6}\right]^{3+}$, observa-se o decréscimo da distância de ligação C-O, de 1,147 para 1,118 ̊, no sentido em que ocorre o aumento do estado de oxidação do metal. Esta tendência deve estar relacionada com a capacidade do centro metálico de promover a retrodoação para o ligante $(\mathrm{CO})$, sendo que se espera que tal capacidade diminua conforme aumente o estado de oxidação do metal (CRABTREE, 2005).

\subsection{Propriedades espectroscópicas}

As frequências vibracionais das bandas correspondentes aos estiramentos CO concordam satisfatoriamente com as obtidas experimentalmente, com um desvio máximo de $43 \mathrm{~cm}^{-1}$ para o estiramento CO assimétrico do tetracarbonilníquel (0), como mostrado na Tabela 13. A maioria dos complexos metálicos estudados apresentam frequências de estiramento dos ligantes mais baixas em relação aos ligantes livres, exibindo, assim, um deslocamento das bandas $\mathrm{CO}$ e $\mathrm{CN}$ para o vermelho. Em alguns casos, todavia, observa-se um deslocamento para o azul, referentes aos complexos catiônicos $\left[\mathrm{Fe}(\mathrm{CO})_{6}\right]^{2+}$ e $\left[\mathrm{Co}(\mathrm{CO})_{6}\right]^{3+}$ e aniônicos $\left[\mathrm{Zn}(\mathrm{CN})_{4}\right]^{2-}$.

Tabela 13 - Frequências vibracionais $\left(\mathrm{cm}^{-1}\right)$ e intensidades fundamentais de infravermelho $\left(\mathrm{km} \mathrm{mol}^{-1}\right)$ dos modos de estiramento $\mathrm{CO}$ e $\mathrm{CN}$ dos ligantes livres e dos complexos metálicos, calculadas diretamente em nível de cálculo B2PLYP/aug-cc-pVTZ e obtidas por meio do modelo CCFDF/QTAIM

\begin{tabular}{llcrrrr}
\hline \multirow{2}{*}{ Sistema } & \multirow{2}{*}{ Modo vibracional $^{\mathrm{a}}$} & \multicolumn{2}{c}{ B2PLYP/aug-cc-pVTZ } & CCFDF/QTAIM & \multicolumn{2}{c}{ Exp. } \\
\cline { 3 - 7 } & & Freq. & Int. & Int. & Freq. & Int. \\
\hline $\mathrm{CO}$ & $\mathrm{CO}$ & 2154 & 60,0 & 60,1 & $2170^{\mathrm{b}}$ & \\
\hline $\mathrm{CN}^{-}$ & $\mathrm{CN}$ & 2060 & 13,2 & 13,2 & & \\
\hline \multirow{2}{*}{$\mathrm{Ni}(\mathrm{CO})_{4}$} & $<\mathrm{CO}>$ (as) & 2052 & 947,7 & 945,7 & $2092^{\mathrm{c}}$ & \\
& $\mathrm{CO}(\mathrm{s})$ & 2126 & 0,0 & 0,0 & $2154^{\mathrm{c}}$ & \\
\hline
\end{tabular}




\begin{tabular}{|c|c|c|c|c|c|c|}
\hline \multirow{2}{*}{ Sistema } & \multirow{2}{*}{ Modo vibracional $^{\mathrm{a}}$} & \multicolumn{2}{|c|}{ B2PLYP/aug-cc-pVTZ } & \multirow{2}{*}{$\begin{array}{r}\text { CCFDF/QTAIM } \\
\text { Int. }\end{array}$} & \multicolumn{2}{|c|}{ Exp. } \\
\hline & & Freq. & Int. & & Freq. & Int. \\
\hline \multirow[t]{4}{*}[\mathrm{Mn}(\mathrm{CO})_{5}]{$^{+}$} & $\mathrm{CO}_{\text {Axial }}$ & 2052 & 528,0 & 565,8 & $2086^{\mathrm{d}}$ & \\
\hline & $<\mathrm{CO}_{\text {Equatorial }}>$ (as) & 2093 & 1118,7 & 1097,1 & $2114^{\mathrm{d}}$ & \\
\hline & $\mathrm{CO}(\mathrm{s})$ fora de fase & 2128 & 0,0 & 0,2 & & \\
\hline & $\mathrm{CO}(\mathrm{s})$ em fase & 2171 & 3,0 & 5,3 & & \\
\hline \multirow[t]{4}{*}{$\mathrm{Fe}(\mathrm{CO})_{5}$} & $\mathrm{CO}_{\text {Axial }}$ (as) & 2006 & 1309,0 & 1405,8 & $2013^{\mathrm{e}}$ & \\
\hline & $<\mathrm{CO}_{\text {Equatorial }}>$ (as) & 2028 & 874,0 & 887,5 & $2034^{e}$ & \\
\hline & $\mathrm{CO}_{\text {Equatorial }}(\mathrm{s})$ fora de fase & 2114 & 0,0 & 0,5 & & \\
\hline & $\mathrm{CO}_{\text {Equatorial }}(\mathrm{s}) \mathrm{em}$ fase & 2036 & 0,0 & 2,8 & & \\
\hline \multirow[t]{3}{*}[\mathrm{Co}(\mathrm{CO})_{6}]{$^{3+}$} & $\langle\mathrm{CO}\rangle$ (as) & 2247 & 23,6 & 24,2 & & \\
\hline & $\langle\mathrm{CO}\rangle(\mathrm{s})$ fora de fase & 2257 & 0,0 & 0,0 & & \\
\hline & $\langle\mathrm{CO}\rangle(\mathrm{s})$ fase & 2255 & 0,0 & 0,0 & & \\
\hline \multirow[t]{3}{*}[\mathrm{Fe}(\mathrm{CO})_{6}]{$^{2+}$} & $<\mathrm{CO}>$ (as) & 2192 & 322,2 & 322,6 & $2209^{f}$ & \\
\hline & $\langle\mathrm{CO}\rangle(\mathrm{s})$ fora de fase & 2210 & 0,0 & 0,0 & & \\
\hline & $<\mathrm{CO}\rangle$ (s) fase & 2225 & 0,0 & 0,0 & & \\
\hline \multirow[t]{3}{*}[\mathrm{Mn}(\mathrm{CO})_{6}]{$^{+}$} & $\langle\mathrm{CO}\rangle$ (as) & 2091 & 1085,3 & 1081,5 & $2115^{\mathrm{d}}$ & \\
\hline & $<\mathrm{CO}\rangle$ (s) fora de fase & 2129 & 0,0 & 0,0 & & \\
\hline & $<\mathrm{CO}\rangle(\mathrm{s})$ fase & 2147 & 0,0 & 0,0 & & \\
\hline \multirow[t]{3}{*}{$\mathrm{Cr}(\mathrm{CO})_{6}$} & $\langle\mathrm{CO}\rangle$ (as) & 1988 & 2062,0 & 2063,8 & $2003^{g}$ & \\
\hline & $<\mathrm{CO}\rangle$ (s) fora de fase & 2033 & 0,0 & 0,0 & & \\
\hline & $\langle\mathrm{CO}\rangle$ (s) fase & 2116 & 0,0 & 0,0 & & \\
\hline \multirow[t]{2}{*}[\mathrm{Zn}(\mathrm{CN})_{4}]{$^{2-}$} & $\langle\mathrm{CN}\rangle$ (as) & 2146 & 7,3 & 8,4 & & \\
\hline & $\mathrm{CN}(\mathrm{s})$ & 2147 & 0,0 & 0,0 & & \\
\hline
\end{tabular}

a Os estiramentos $\mathrm{CO}$ e $\mathrm{CN}$ simétricos e assimétricos são designados por (s) e (as), respectivamente, e, para bandas degeneradas, as médias das propriedades espectroscópicas foram apresentadas. Além disso, os estiramentos axiais e equatoriais foram discriminados de acordo com o fato de estarem em fase ou não entre si.

b NATIONAL INSTITUTE OF STANDARDS E TECHNOLOGY. Chemistry webbook. U. S. Secretary of Commerce: the standard reference data act. 1968. Disponível em: <http//www.nist.gov/index.html>. Acesso em: 05 set. 2019.

c (FAN e ZIEGLER, 1992).

d (REED e DUNCAN, 2010).

e (JONES, MCDOWELL e GOLDBLATT, 1972).

f (GEIER et al., 2007).

g (HANSFORD e DAVIES, 1994).

As frequências vibracionais dos estiramentos $\mathrm{CO}$ tendem a aumentar no sentido em que ocorre o aumento do número de oxidação do metal para a série de complexos octaédricos $\mathrm{Cr}(\mathrm{CO})_{6}<\left[\mathrm{Mn}(\mathrm{CO})_{6}\right]^{+}<\left[\mathrm{Fe}(\mathrm{CO})_{6}\right]^{2+}<\left[\mathrm{Co}(\mathrm{CO})_{6}\right]^{3+}$. Novamente, isto está de acordo com a expectativa de que a capacidade de retrodoação diminua conforme aumente o número de oxidação (NOX) do metal. Além disto, as frequências de modos de estiramento de ligantes $\mathrm{CO}$ axiais nos complexos $\left[\mathrm{Mn}(\mathrm{CO})_{5}\right]^{+}$e $\mathrm{Fe}(\mathrm{CO})_{5}$ são menores que aquelas dos modos dos 
ligantes $\mathrm{CO}$ equatoriais, o que sugere maior retrodoação para a posição axial. Considerando os modos ativos na espectroscopia de infravermelho, a banda correspondente ao estiramento CO do complexo neutro tende a ter um deslocamento maior em relação ao ligante livre, de cerca de $166 \mathrm{~cm}^{-1}$, do que aqueles observados para as espécies catiônicas e a direção deste deslocamento se modifica do vermelho para o azul ao longo da série.

Por sua vez, as intensidades de infravermelho dos estiramentos $\mathrm{CO}$ e $\mathrm{CN}$ calculadas segundo o modelo CCFDF/QTAIM diferem em, no máximo, $96,9 \mathrm{~km} \mathrm{~mol}^{-1}$, quando comparadas aos resultados obtidos diretamente em nível B2PLYP/aug-cc-pVTZ. De modo geral, as bandas CO e CN tornam-se bem mais intensas por conta da complexação, de 45,9 a $2002,0 \mathrm{~km} \mathrm{~mol}^{-1}$. Observa-se também que o valor desta propriedade diminui ao longo da série $\mathrm{Cr}(\mathrm{CO})_{6}>\left[\mathrm{Mn}(\mathrm{CO})_{6}\right]^{+}>\left[\mathrm{Fe}(\mathrm{CO})_{6}\right]^{2+}>\left[\mathrm{Co}(\mathrm{CO})_{6}\right]^{3+}$.

A análise de carga, fluxo de carga, fluxo de dipolo e de seus termos cruzados para as intensidades de infravermelho dos modos de estiramento $\mathrm{CO}$ e $\mathrm{CN}$, segundo o modelo CCFDF/QTAIM, é apresentada na Tabela 14. Como alguns dos estiramentos CO e CN de cada complexo se acoplam durante os movimentos vibracionais, as variações das intensidades de infravermelho $(\Delta A)$ foram calculadas subtraindo-se a soma dos valores desta propriedade para todos os estiramentos $\mathrm{CO}$ ou $\mathrm{CN}$ de um dado complexo, $\left(\sum A_{\text {est_lig }}\right)_{\text {complexo }}$, em relação àquela dos respectivos ligantes livres, $A_{\text {est_lig_livre }}, \Delta A^{\prime}=\left[\left(\sum A_{\text {est_lig }}\right)_{\text {complexo }}-\right.$ $\left.n\left(A_{\text {est_lig_livre }}\right)\right]$, na qual $n$ é o número de ligantes no complexo. Além disso, para se obter o efeito da complexação na intensidade de infravermelho por unidade de ligante, dividiu-se $\Delta A$, pelo número de ligantes $\mathrm{CO}$ ou $\mathrm{CN}^{-}$presentes no sistema, $\Delta A=\left[\left(\sum A_{\text {est_lig }}\right)_{\text {complexo }}\right.$ $\left.n\left(A_{\text {est_lig_livre }}\right)\right] / n$.

Tabela 14 - Variações das contribuições de carga, fluxo de carga, fluxo de dipolo e os termos cruzados entre estas quantidades durante a complexação por unidade de ligante para a intensidade total, $A,\left(\mathrm{~km} \mathrm{~mol}^{-1}\right)$ dos modos de estiramentos $\mathrm{CO}$ e $\mathrm{CN}$ segundo o modelo CCFDF/QTAIM

\begin{tabular}{lccccccc}
\hline \multirow{2}{*}{ Sistema } & \multicolumn{7}{c}{ CCFDF/QTAIM $^{\mathrm{a}}$} \\
\cline { 2 - 8 } & $\Delta A^{C}$ & $\Delta A^{C F}$ & $\Delta A^{D F}$ & $\Delta A^{C \times C F}$ & $\Delta A^{C \times D F}$ & $\Delta A^{C F \times D F}$ & $\Delta A^{\text {Total }}$ \\
\hline $\mathrm{Ni}(\mathrm{CO})_{4}$ & $-55,3$ & $-187,2$ & $-11,3$ & 582,7 & $-53,5$ & 373,9 & 649,2 \\
{$\left[\mathrm{Mn}(\mathrm{CO})_{5}\right]^{+}$} & $-42,9$ & $-209,4$ & 32,4 & 436,4 & 9,7 & 266,8 & 493,0 \\
$\mathrm{Fe}(\mathrm{CO})_{5}$ & $-53,4$ & $-221,4$ & 32,2 & 495,8 & 0,9 & 326,5 & 580,7 \\
{$\left[\mathrm{Co}(\mathrm{CO})_{6}\right]^{3+}$} & $-35,5$ & 245,0 & 78,5 & $-129,2$ & 70,5 & $-277,4$ & $-48,0$ \\
{$\left[\mathrm{Fe}(\mathrm{CO})_{6}\right]^{2+}$} & $-34,3$ & $-112,9$ & 36,7 & 150,1 & 25,6 & 36,1 & 101,2 \\
{$\left[\mathrm{Mn}(\mathrm{CO})_{6}\right]^{+}$} & $-49,7$ & $-272,4$ & 5,7 & 561,4 & $-25,0$ & 356,8 & 576,9
\end{tabular}




\begin{tabular}{lccccccc}
\hline \multirow{2}{*}{ Sistema } & $\Delta A^{C}$ & $\Delta A^{C F}$ & $\Delta A^{D F}$ & $\Delta A^{C \times C F}$ & $\Delta A^{C \times D F}$ & $\Delta A^{C F \times D F}$ & $\Delta A^{\text {Total }}$ \\
\cline { 2 - 8 } & $-50,7$ & $-83,6$ & $-15,6$ & 720,4 & $-56,9$ & 458,2 & 971,9 \\
$\mathrm{Cr}(\mathrm{CO})_{6}$ & $-3,3$ & 02,5 & 28,0 & $-31,8$ & 9,7 & $-112,0$ & $-7,0$ \\
{$\left[\mathrm{Zn}(\mathrm{CN})_{4}\right]^{2-}$} & ${ }^{\mathrm{a}} \Delta A=\left[\left(\sum A_{\text {est_lig }}\right)_{\text {complexo }}-n\left(A_{\text {est_lig_livre }}\right)\right] / n$. & & &
\end{tabular}

O aumento da intensidade de infravermelho ou a sua diminuição (em alguns poucos casos) durante a complexação podem ser explicados pela contribuição cruzada entre a carga e o fluxo de carga, $\Delta A^{\mathrm{C} \times \mathrm{CF}}$, como mostrado na Figura 14. Isto significa que é a transferência de carga eletrônica e o seu reflexo no fluxo desta quantidade que explica boa parte da variação da intensidade total por unidade. Aparentemente, o aumento da capacidade de retrodoação do centro metálico contribui para o aumento das intensidades de estiramento $\mathrm{CO}$, como visto na Tabela 14 para a série $\mathrm{Cr}(\mathrm{CO})_{6}>\left[\mathrm{Mn}(\mathrm{CO})_{6}\right]^{+}>\left[\mathrm{Fe}(\mathrm{CO})_{6}\right]^{2+}>\left[\mathrm{Co}(\mathrm{CO})_{6}\right]^{3+}$. Além disto, a intensidade por ligante parece sempre diminuir com o aumento do número de oxidação do centro metálico nas carbonilas. Neste caso, os valores variam entre 581 e $972 \mathrm{~km} \mathrm{~mol}^{-1}$ para centros com NOX $=0$, de 493 a $577 \mathrm{~km} \mathrm{~mol}^{-1}$ para $\mathrm{NOX}=1$, ao redor de $101 \mathrm{~km} \mathrm{~mol}^{-1}$ para $\mathrm{NOX}=2 \mathrm{e}-48 \mathrm{~km} \mathrm{~mol}^{-1}$ para $\mathrm{NOX}=3$.

Figura 14 - Variação da intensidade de infravermelho total dos modos de estiramento CO e $\mathrm{CN}$ durante a complexação por unidade de ligante, $\Delta A$, em função da contribuição cruzada entre a carga e o fluxo de carga, $\Delta A^{\mathrm{C} \times \mathrm{CF}}\left(\mathrm{em} \mathrm{km} \mathrm{mol}^{-1}\right)$

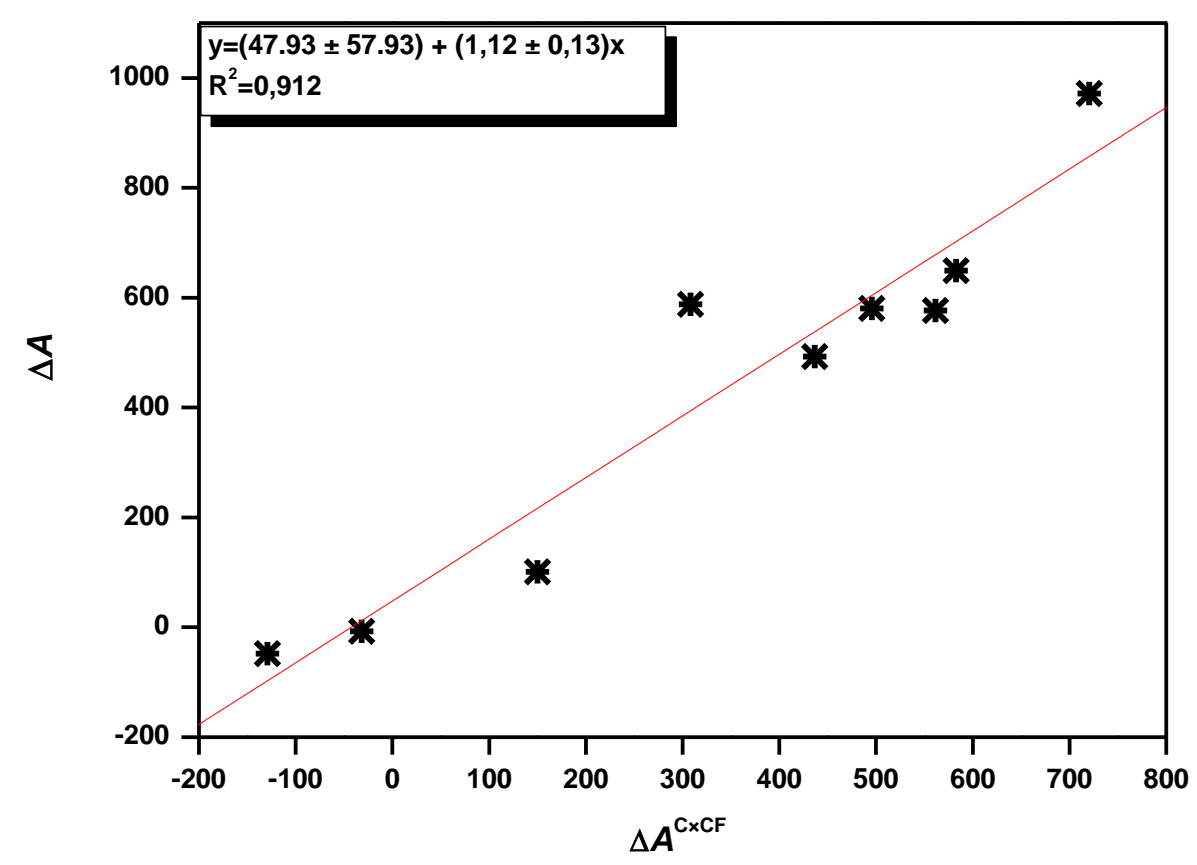




\subsection{Propriedades topológicas QTAIM}

A Tabela 15 mostra as médias dos valores das propriedades topológicas da densidade eletrônica nos pontos críticos das ligações entre o metal e o ligante. As médias das densidades eletrônicas variam de 0,084 a 0,156 u. a. Como esperado, estes valores são maiores que aqueles apresentados no capítulo anterior para ligações de hidrogênio em dímeros de ácidos carboxílicos $\left(\rho_{B C P}=0,04\right.$ a 0,06 u. a.). Os maiores valores para a densidade eletrônica são observados para as ligações axiais Fe-C e Mn-C ( $\left\langle\rho_{B C P}\right\rangle=0,156$ e 0,155 u.a., respectivamente) das pentacarbonilas. $\mathrm{O}$ incremento de $\rho$ no ponto crítico de ligação, juntamente com o decréscimo da densidade de energia total, são indicadores de aumento do caráter covalente das ligações. A Figura 15 evidencia uma correlação linear entre as duas quantidades mencionadas $\left(\mathrm{R}^{2}=0,915\right)$ nos BCPs metal - ligante. Por sua vez, a soma das curvaturas $\lambda_{1}, \lambda_{2} \mathrm{e}$ $\lambda_{3}$ fornece o Laplaciano da densidade eletrônica e, para os complexos considerados neste estudo, os valores do Laplaciano são todos positivos, o que sugere certo caráter iônico das ligações entre o metal e o ligante. Tais características topológicas são comuns em BCPs do tipo metal - ligante (CORTÉS-GUZMÁN e BADER, 2005).

Tabela 15 - Médias das propriedades QTAIM nos pontos críticos das ligações entre o metal e o ligante calculadas em nível B2PLYP/aug-cc-pVTZ (em u. a.)

\begin{tabular}{llrrrrrrrr}
\hline Sistema & Ligação & $\left\langle\rho_{B C P}\right\rangle$ & $\left\langle\Delta \lambda_{1}\right\rangle$ & $\left\langle\Delta \lambda_{2}\right\rangle$ & $\left\langle\Delta \lambda_{3}\right\rangle$ & $\left\langle\nabla^{2} \rho_{B C P}\right\rangle$ & $\left\langle V_{B C P}\right\rangle$ & $\left\langle G_{B C P}\right\rangle$ & $\left\langle H_{B C P}=\left(G_{B C P}+V_{B C P}\right)\right\rangle$ \\
\hline $\mathrm{Ni}(\mathrm{CO})_{4}$ & $\mathrm{NiC}$ & 0,132 & $-0,165$ & $-0,165$ & 0,800 & 0,470 & $-0,239$ & 0,178 & $-0,061$ \\
{$\left[\mathrm{Mn}(\mathrm{CO})_{5}\right]^{+}$} & $\mathrm{MnC}_{\text {Axial }}$ & 0,155 & $-0,157$ & $-0,157$ & 0,842 & 0,529 & $-0,271$ & 0,202 & $-0,069$ \\
& $\mathrm{MnC}_{\text {Equatorial }}$ & 0,120 & $-0,119$ & $-0,113$ & 0,706 & 0,474 & $-0,202$ & 0,160 & $-0,042$ \\
$\mathrm{Fe}(\mathrm{CO})_{5}$ & $\mathrm{FeC}_{\text {Axial }}$ & 0,156 & $-0,204$ & $-0,204$ & 0,865 & 0,458 & $-0,270$ & 0,192 & $-0,078$ \\
& $\mathrm{FeC}$ Equatorial & 0,143 & $-0,193$ & $-0,184$ & 0,830 & 0,453 & $-0,247$ & 0,180 & $-0,067$ \\
{$\left[\mathrm{Co}(\mathrm{CO})_{6}\right]^{3+}$} & $\mathrm{CoC}$ & 0,114 & $-0,122$ & $-0,122$ & 0,553 & 0,309 & $-0,170$ & 0,124 & $-0,046$ \\
{$\left[\mathrm{Fe}(\mathrm{CO})_{6}\right]^{2+}$} & $\mathrm{FeC}$ & 0,124 & $-0,126$ & $-0,126$ & 0,678 & 0,426 & $-0,204$ & 0,155 & $-0,049$ \\
{$\left[\mathrm{Mn}(\mathrm{CO})_{6}\right]^{+}$} & $\mathrm{MnC}$ & 0,125 & $-0,132$ & $-0,132$ & 0,728 & 0,464 & $-0,207$ & 0,162 & $-0,046$ \\
$\mathrm{Cr}(\mathrm{CO})_{6}$ & $\mathrm{CrC}$ & 0,113 & $-0,117$ & $-0,117$ & 0,693 & 0,459 & $-0,183$ & 0,149 & $-0,034$ \\
{$\left[\mathrm{Zn}(\mathrm{CN})_{4}\right]^{2-}$} & $\mathrm{ZnC}$ & 0,084 & $-0,100$ & $-0,100$ & 0,436 & 0,237 & $-0,115$ & 0,087 & $-0,028$ \\
\hline
\end{tabular}


Figura 15 - Correlação entre as médias da densidade de energia total, $H_{B C P}$, e da densidade eletrônica, $\rho_{B C P}$, nos pontos críticos das ligações entre o metal e o ligante (em u. a.)

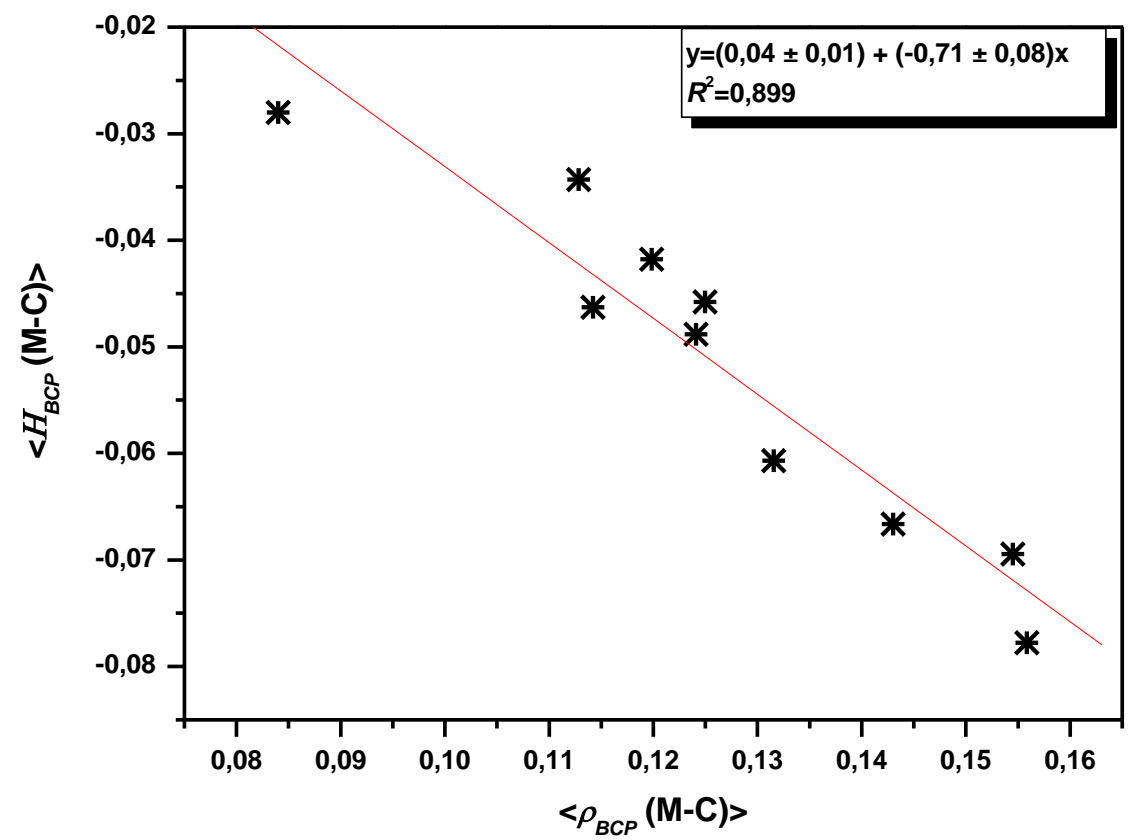

Por sua vez, as variações das propriedades topológicas da densidade eletrônica nos pontos críticos das ligações $\mathrm{C}-\mathrm{O}$ ou $\mathrm{C}-\mathrm{N}$, em relação aos ligantes livres, são apresentadas na Tabela 16. De modo geral, a complexação leva a uma diminuição da densidade eletrônica, exceto para os sistemas $\left[\mathrm{Fe}(\mathrm{CO})_{6}\right]^{2+},\left[\mathrm{Co}(\mathrm{CO})_{6}\right]^{3+} \mathrm{e}\left[\mathrm{Zn}(\mathrm{CN})_{4}\right]^{2-}$, nos quais ocorre um aumento de $\rho_{B C P}$. As mudanças desta propriedade parecem indicar um enfraquecimento da ligação CO durante o processo de coordenação do ligante ao metal. Além disso, o aumento na capacidade de retrodoação do centro metálico com a redução do NOX leva a menores valores de $\rho_{B C P}$ e de $\Delta \nabla^{2} \rho_{B C P}$, juntamente com maiores valores de $H_{B C P}$ dos ligantes, como visto na série isoeletrônica $\mathrm{Cr}(\mathrm{CO})_{6},\left[\mathrm{Mn}(\mathrm{CO})_{6}\right]^{+},\left[\mathrm{Fe}(\mathrm{CO})_{6}\right]^{2+}$ e $\left[\mathrm{Co}(\mathrm{CO})_{6}\right]^{3+}$. A alteração nas quantidades topológicas dos ligantes durante a complexação também parece estar associada com o NOX do centro metálico nos demais casos. Por exemplo, no caso de $\Delta \rho_{B C P}$ em carbonilas temos valores entre $-0,109$ e $-0,042$ u. a. para $\operatorname{NOX}=0$, de $-0,016$ a $-0,005$ u. a. para NOX $=1$, 0,060 u. a. para $\mathrm{NOX}=2$, e 0,087 u. a. para $\mathrm{NOX}=3$.

Tabela 16 - Variações das propriedades QTAIM nos pontos críticos das ligações CO e CN nos complexos de coordenação estudados em relação aos ligantes livres, calculadas em nível B2PLYP/aug-cc-pVTZ (em u. a.)

\begin{tabular}{llrrrrrrrr}
\hline Sistema & Ligação & $\Delta \rho_{B C P}$ & $\Delta \lambda_{1}$ & $\Delta \lambda_{2}$ & $\Delta \lambda_{3}$ & $\Delta \nabla^{2} \rho_{B C P}$ & $\Delta V_{B C P}$ & $\Delta G_{B C P}$ & $\Delta H_{B C P}=\left(\Delta G_{B C P}+\Delta V_{B C P}\right)$ \\
\hline $\mathrm{Ni}(\mathrm{CO})_{4}$ & $\mathrm{CO}$ & $-0,052$ & 0,665 & 0,665 & $-1,807$ & $-0,478$ & 0,318 & $-0,219$ & 0,099
\end{tabular}




\begin{tabular}{llrrrrrrrr}
\hline Sistema & Ligação & $\Delta \rho_{B C P}$ & $\Delta \lambda_{1}$ & $\Delta \lambda_{2}$ & $\Delta \lambda_{3}$ & $\Delta \nabla^{2} \rho_{B C P}$ & $\Delta V_{B C P}$ & $\Delta G_{B C P}$ & $\Delta H_{B C P}=\left(\Delta G_{B C P}+\Delta V_{B C P}\right)$ \\
\hline$\left[\mathrm{Mn}(\mathrm{CO})_{5}\right]^{+}$ & $\mathrm{CO}_{\text {Axial }}$ & $-0,012$ & 0,235 & 0,235 & $-0,539$ & $-0,070$ & 0,062 & $-0,040$ & 0,022 \\
& $\mathrm{CO}_{\text {Equatorial }}$ & $-0,005$ & 0,594 & 0,608 & $-1,102$ & 0,100 & $-0,055$ & 0,040 & $-0,015$ \\
$\mathrm{Fe}(\mathrm{CO})_{5}$ & $\mathrm{CO}_{\text {Axial }}$ & $-0,042$ & 0,522 & 0,522 & $-1,362$ & $-0,317$ & 0,256 & $-0,168$ & 0,089 \\
& $\mathrm{CO}$ Equatorial & $-0,059$ & 0,669 & 0,722 & $-1,824$ & $-0,432$ & 0,356 & $-0,232$ & 0,124 \\
{$\left[\mathrm{Co}(\mathrm{CO})_{6}\right]^{3+}$} & $\mathrm{CO}$ & 0,087 & 1,013 & 1,013 & 0,274 & 2,300 & $-1,063$ & 0,819 & $-0,244$ \\
{$\left[\mathrm{Fe}(\mathrm{CO})_{6}\right]^{2+}$} & $\mathrm{CO}$ & 0,060 & 0,835 & 0,835 & $-0,524$ & 1,145 & $-0,664$ & 0,475 & $-0,189$ \\
{$\left[\mathrm{Mn}(\mathrm{CO})_{6}\right]^{+}$} & $\mathrm{CO}$ & $-0,016$ & 0,998 & 0,998 & $-1,930$ & 0,066 & $-0,024$ & 0,020 & $-0,004$ \\
$\mathrm{Cr}(\mathrm{CO})_{6}$ & $\mathrm{CO}$ & $-0,109$ & 1,242 & 1,242 & $-3,381$ & $-0,897$ & 0,672 & $-0,448$ & 0,224 \\
{$\left[\mathrm{Zn}(\mathrm{CN})_{4}\right]^{2-}$} & $\mathrm{CN}$ & 0,034 & 1,113 & 1,113 & 0,130 & 0,357 & $-0,294$ & 0,191 & $-0,102$ \\
\hline
\end{tabular}

\subsection{Análise de Átomos Quânticos Interagentes}

A Tabela 17 mostra a partição energética IQA para a energia interatômica das ligações entre o metal e o carbono, carbono e oxigênio e carbono e nitrogênio. A energia de interação metal-carbono das hexacarbonilas metálicas é positiva (desestabilizante) e dominada pelo efeito eletrostático clássico. Esta contribuição clássica desestabilizante (predominantemente iônica) aumenta na seguinte ordem $\mathrm{Cr}(\mathrm{CO})_{6}<\left[\mathrm{Mn}(\mathrm{CO})_{6}\right]^{+}<\left[\mathrm{Fe}(\mathrm{CO})_{6}\right]^{2+}<\left[\mathrm{Co}(\mathrm{CO})_{6}\right]^{3+}$. Características semelhantes são observadas para as ligação $\mathrm{MnC}_{\mathrm{Axial}}$ e $\mathrm{MnC}_{\text {Equatorial do }}$ complexo $\left[\mathrm{Mn}(\mathrm{CO})_{5}\right]^{+}$, respectivamente, ou seja, $E_{\text {Inter }}>0$ e $V_{C l}>V_{X C}$. A contribuição estabilizante de troca-correlação, por sua vez, é predominante para a maioria das ligações metal-carbono das pentacarbonilas e para as ligações $\mathrm{NiC}$ e $\mathrm{ZnC}$ dos sistemas $\mathrm{Ni}(\mathrm{CO})_{4}$ e $\left[\mathrm{Zn}(\mathrm{CN})_{4}\right]^{2-}$.

Tabela 17 - Médias das energias de interação IQA $\left(\left\langle E_{\text {Inter }}\right\rangle\right)$ juntamente com os componentes clássico $\left(V_{c l}\right)$ e de troca-correlação $\left(V_{X C}\right)$ para as ligações metal-carbono, carbono-oxigênio e carbono-nitrogênio dos ligantes livres e dos complexos de coordenação, calculadas em nível B2PLYP/aug-cc-pVTZ (em u. a.)

\begin{tabular}{llrrr}
\hline Sistema & Ligação & $\left\langle E_{\text {Inter }}\left(\Omega, \Omega^{\prime}\right)\right\rangle$ & $\left\langle V_{c l}\left(\Omega, \Omega^{\prime}\right)\right\rangle$ & $\left\langle V_{X C}\left(\Omega, \Omega^{\prime}\right)\right\rangle$ \\
\hline $\mathrm{CO}$ livre & $\mathrm{CO}$ & $-1,7007$ & $-1,2371$ & $-0,4637$ \\
\hline $\mathrm{CN}^{-}$livre & $\mathrm{CN}$ & $-1,4599$ & $-0,8899$ & $-0,5699$ \\
\hline $\mathrm{Ni}(\mathrm{CO})_{4}$ & $\langle\mathrm{NiC}\rangle$ & $-0,0664$ & 0,1534 & $-0,2198$ \\
& $\langle\mathrm{CO}\rangle$ & $-1,4973$ & $-1,0521$ & $-0,4452$ \\
\hline$\left[\mathrm{Mn}(\mathrm{CO})_{5}\right]^{+}$ & $\mathrm{MnC}_{\text {Axial }}$ & $-0,0254$ & 0,2389 & $-0,2644$ \\
& $\mathrm{CO}_{\text {Axial }}$ & $-1,4587$ & $-1,0048$ & $-0,4539$ \\
& $\left\langle\mathrm{MnC}_{\text {Equatorial }}\right\rangle$ & 0,0348 & 0,2280 & $-0,1931$ \\
& $\left\langle\mathrm{CO}_{\text {Equatorial }}\right\rangle$ & $-1,5089$ & $-1,0455$ & $-0,4634$ \\
\hline
\end{tabular}




\begin{tabular}{llrrr}
\hline Sistema & Ligação & $\left\langle E_{\text {Inter }}\left(\Omega, \Omega^{\prime}\right)\right\rangle$ & $\left\langle V_{c 1}\left(\Omega, \Omega^{\prime}\right)\right\rangle$ & $\left\langle V_{X C}\left(\Omega, \Omega^{\prime}\right)\right\rangle$ \\
\hline $\mathrm{Fe}(\mathrm{CO})_{5}$ & $\langle\mathrm{FeC}$ Axial & $-0,0492$ & 0,1964 & $-0,2456$ \\
& $\left\langle\mathrm{CO}_{\text {Axial }}\right\rangle$ & $-1,4483$ & $-1,0071$ & $-0,4412$ \\
& $\left\langle\mathrm{FeC}_{\text {Equatorial }}\right\rangle$ & $-0,0456$ & 0,1813 & $-0,2268$ \\
& $\langle\mathrm{CO}$ Equatorial & $-1,4588$ & $-1,0165$ & $-0,4424$ \\
\hline$\left[\mathrm{Co}(\mathrm{CO})_{6}\right]^{3+}$ & $\langle\mathrm{CoC}\rangle$ & 0,0720 & 0,2429 & $-0,1709$ \\
& $\langle\mathrm{CO}\rangle$ & $-1,5276$ & $-1,0247$ & $-0,5029$ \\
\hline$\left[\mathrm{Fe}(\mathrm{CO})_{6}\right]^{2+}$ & $\langle\mathrm{FeC}\rangle$ & 0,0385 & 0,2335 & $-0,1951$ \\
& $\langle\mathrm{CO}\rangle$ & $-1,5407$ & $-1,0587$ & $-0,4821$ \\
\hline$\left[\mathrm{Mn}(\mathrm{CO})_{6}\right]^{+}$ & $\langle\mathrm{MnC}\rangle$ & 0,0379 & 0,2321 & $-0,1943$ \\
& $\langle\mathrm{CO}\rangle$ & $-1,5095$ & $-1,0481$ & $-0,4614$ \\
\hline $\mathrm{Cr}(\mathrm{CO})_{6}$ & $\langle\mathrm{CrC}\rangle$ & 0,0417 & 0,2154 & $-0,1736$ \\
& $\langle\mathrm{CO}\rangle$ & $-1,4685$ & $-1,0250$ & $-0,4436$ \\
\hline$\left[\mathrm{Zn}(\mathrm{CN})_{4}\right]^{2-}$ & $\langle\mathrm{ZnC}\rangle$ & $-0,0222$ & 0,0973 & $-0,1195$ \\
& $\langle\mathrm{CN}\rangle$ & $-1,4304$ & $-0,8517$ & $-0,5787$ \\
\hline
\end{tabular}

Já as energias interatômicas dos ligantes $\mathrm{CO}$ e $\mathrm{CN}^{-}$nos complexos são sempre menos negativas (estabilizantes) do que as dos respectivos ligantes livres. No caso de $E_{\text {Inter }}(\mathrm{C}, \mathrm{O})$, nas hexacarbonilas, observamos que $\mathrm{Cr}(\mathrm{CO})_{6}>\left[\mathrm{Mn}(\mathrm{CO})_{6}\right]^{+}>\left[\mathrm{Fe}(\mathrm{CO})_{6}\right]^{2+}$, ou seja, a desestabilização da ligação CO destes complexos está em acordo com o esperado por meio do efeito de retrodoação. Neste caso, o complexo $\left[\mathrm{Co}(\mathrm{CO})_{6}\right]^{3+}$ parece ser uma exceção. Porém, ao analisarmos somente as contribuições não-clássicas (predominantemente covalentes) para $E_{\text {Inter }}(\mathrm{C}, \mathrm{O})$, observamos claramente que $\mathrm{Cr}(\mathrm{CO})_{6}>\left[\mathrm{Mn}(\mathrm{CO})_{6}\right]^{+}>\left[\mathrm{Fe}(\mathrm{CO})_{6}\right]^{2+}>\left[\mathrm{Co}(\mathrm{CO})_{6}\right]^{3+}$, ou seja, valores mais negativos para $V_{X C}(\mathrm{C}, \mathrm{O})$, que indicam maior caráter estabilizante covalente, são obtidos no complexo $\left[\mathrm{Co}(\mathrm{CO})_{6}\right]^{3+}$.

Além disto, variações similares são observadas nos termos da Tabela 17 de acordo com o NOX de cada centro metálico. Por exemplo, analisando as variações em relação ao ligante livre, $\Delta V_{X C}(\mathrm{C}, \mathrm{O})$ varia entre 0,019 e 0,023 u. a. para $\mathrm{NOX}=0$, de 0,000 a 0,010 u. a. para $\operatorname{NOX}=1$, ao redor de $-0,018$ para $\mathrm{NOX}=2$ e aproximadamente $-0,039$ u. a. para NOX $=3$. Finalmente, comparando as posições axiais e equatoriais dos complexos $\left[\mathrm{Mn}(\mathrm{CO})_{5}\right]^{+} \mathrm{e}$ $\mathrm{Fe}(\mathrm{CO})_{5}$, nota-se, principalmente no primeiro, uma maior desestabilização do termo $V_{X C}(\mathrm{C}, \mathrm{O})$ nas posições axiais, o que sugere maior retrodoação do centro metálico para estes ligantes.

A interação metal-ligante (M-L) nos complexos estudados, ou seja, a soma das energias de interação entre o metal e cada átomo do ligante (em valores médios), tende a ser sempre estabilizante devido a maior contribuição do componente de troca-correlação para esta 
energia, como mostrado na Tabela 18. A preponderância de $V_{X C}(\mathrm{M}-\mathrm{L})$ indica que estas ligações possuem um caráter covalente mais acentuado, exceto nos complexos $\mathrm{Ni}(\mathrm{CO})_{4}$ $\left[\mathrm{Zn}(\mathrm{CN})_{4}\right]^{2-}$. Além disto, a contribuição estabilizante da energia de interação metal-ligante e de seu componente eletrostático tendem a aumentar segundo a sequência $\mathrm{Cr}(\mathrm{CO})_{6}<$ $\left[\mathrm{Mn}(\mathrm{CO})_{6}\right]^{+}<\left[\mathrm{Fe}(\mathrm{CO})_{6}\right]^{2+}<\left[\mathrm{Co}(\mathrm{CO})_{6}\right]^{3+}$.

Tabela 18 - Valores médios das energias de interação IQA entre o metal e o ligante, assim como os seus componentes eletrostático e de troca-correlação, obtidos em nível de cálculo B2PLYP/aug-cc-pVTZ (em u. a.)

\begin{tabular}{lrrr}
\hline Sistema & $\left\langle E_{\text {Inter }}(\mathrm{M}, \mathrm{L})\right\rangle$ & $\left\langle V_{c l}(\mathrm{M}, \mathrm{L})\right\rangle$ & $\left\langle V_{X C}(\mathrm{M}, \mathrm{L})\right\rangle$ \\
\hline $\mathrm{Ni}(\mathrm{CO})_{4}$ & $-0,2545$ & $-0,0240$ & $-0,2305$ \\
{$\left[\mathrm{Mn}(\mathrm{CO})_{5}\right]^{+}$} & $-0,2279$ & $-0,0102$ & $-0,2178$ \\
$\mathrm{Fe}(\mathrm{CO})_{5}$ & $-0,2665$ & $-0,0204$ & $-0,2460$ \\
{$\left[\mathrm{Co}(\mathrm{CO})_{6}\right]^{3+}$} & $-0,1120$ & 0,0651 & $-0,1771$ \\
{$\left[\mathrm{Fe}(\mathrm{CO})_{6}\right]^{2+}$} & $-0,1690$ & 0,0344 & $-0,2034$ \\
{$\left[\mathrm{Mn}(\mathrm{CO})_{6}\right]^{+}$} & $-0,2096$ & $-0,0064$ & $-0,2033$ \\
$\mathrm{Cr}(\mathrm{CO})_{6}$ & $-0,2427$ & $-0,0608$ & $-0,1819$ \\
{$\left[\mathrm{Zn}(\mathrm{CN})_{4}\right]^{2-}$} & $-0,3042$ & $-0,1805$ & $-0,1237$ \\
\hline
\end{tabular}

\subsection{Análise das variações de cargas atômicas durante a complexação}

As cargas atômicas QTAIM das espécies metálicas e dos carbonos são sempre positivas, enquanto aquelas dos nitrogênios e oxigênios são sempre negativas (Tabela 19). As cargas dos centros metálicos, dos carbonos e dos oxigênios ou nitrogênios variam de 0,749 a $1,247 e$, de 0,645 a $1,218 e$, e de $-1,430$ a $-0,879 e$, respectivamente. As cargas positivas assumidas pelos centros metálicos com NOX 0 indicam claramente que a retrodoação metal ligante é maior que a doação ligante - metal, o que parece também ser observado para os centros metálicos com $\mathrm{NOX}=1$, que assumem cargas maiores que 1 nos complexos estudados. Entretanto, o oposto ocorre para os centros metálicos com $\mathrm{NOX} \geq 2$, de forma que a doação ligante - metal é mais expressiva que a retrodoação nestes casos.

De modo geral, quando carbonilas se coordenam às espécies metálicas consideradas, os carbonos recebem carga eletrônica $(\Delta q=-0,306$ a $-0,028 e)$ e os oxigênios perdem carga eletrônica $(\Delta q=0,035$ a $0,302 e)$, exceto no complexo $\left[\mathrm{Co}(\mathrm{CO})_{6}\right]^{3+}$, o qual o carbono também perde uma pequena fração de carga eletrônica. Além disso, os oxigênios equatoriais são mais negativamente carregados que os axiais nas pentacarbonilas metálicas. Já para o complexo com o ligante $\mathrm{CN}^{-}$, ocorre uma transferência de carga eletrônica de ambos os átomos $(\mathrm{C}$ e $\mathrm{N})$ para o centro metálico. 
Embora seja mais complicado de analisar, por conta de muitos efeitos a serem considerados simultaneamente (efetividade da doação $\sigma$ ligante - metal, mudanças de carga devido às variações de comprimentos de ligação dos ligantes na complexação, entre outros), parece que uma parcela significativa da carga eletrônica recebida pelo carbono do ligante CO advém da retrodoação. Isto explicaria a razão pela qual os complexos contendo centros metálicos com NOX 0 ou 1 exibem as maiores frações de carga eletrônica recebidas por átomos de carbono em carbonilas (entre -0,306 e -0,141e).

Tabela 19 - Cargas atômicas QTAIM dos complexos de coordenação estudados, bem como suas variações em relação aos ligantes livres (em $e$ )

\begin{tabular}{llrr}
\hline Sistema & Átomo & $\langle q(\mathrm{QTAIM})\rangle^{\mathrm{a}}$ & $\Delta q(\mathrm{QTAIM})^{\mathrm{b}}$ \\
\hline $\mathrm{Ni}(\mathrm{CO})_{4}$ & $\mathrm{Ni}$ & 0,749 & \\
& $\mathrm{C}$ & 0,959 & $-0,222$ \\
& $\mathrm{O}$ & $-1,146$ & 0,035 \\
\hline$\left[\mathrm{Mn}(\mathrm{CO})_{5}\right]^{+}$ & $\mathrm{Mn}$ & 1,126 & \\
& $\mathrm{C}_{\text {Axial }}$ & 1,014 & $-0,167$ \\
& $\mathrm{C}_{\text {Equatorial }}$ & 1,040 & $-0,141$ \\
& $\mathrm{O}_{\text {Axial }}$ & $-1,057$ & 0,124 \\
& $\mathrm{O}_{\text {Equatorial }}$ & $-1,073$ & 0,108 \\
\hline $\mathrm{Fe}(\mathrm{CO})_{5}$ & $\mathrm{Fe}$ & 0,892 & \\
& $\mathrm{C}_{\text {Axial }}$ & 0,973 & $-0,207$ \\
& $\mathrm{C}_{\text {Equatorial }}$ & 0,947 & $-0,233$ \\
& $\mathrm{O}_{\text {Axial }}$ & $-1,129$ & 0,052 \\
& $\mathrm{O}_{\text {Equatorial }}$ & $-1,140$ & 0,041 \\
\hline$\left[\mathrm{Co}(\mathrm{CO})_{6}\right]^{3+}$ & $\mathrm{Co}$ & 0,969 & \\
& $\mathrm{C}$ & 1,218 & 0,037 \\
& $\mathrm{O}$ & $-0,879$ & 0,302 \\
\hline$\left[\mathrm{Fe}(\mathrm{CO})_{6}\right]^{2+}$ & $\mathrm{Fe}$ & 0,979 & \\
& $\mathrm{C}$ & 1,153 & $-0,028$ \\
& $\mathrm{O}$ & $-0,982$ & 0,199 \\
\hline$\left[\mathrm{Mn}(\mathrm{CO})_{6}\right]^{+}$ & $\mathrm{Mn}$ & 1,109 & \\
& $\mathrm{C}$ & 0,874 & $-0,306$ \\
& $\mathrm{O}$ & $-1,067$ & 0,114 \\
\hline $\mathrm{Cr}(\mathrm{CO})_{6}$ & $\mathrm{Cr}$ & 1,247 & \\
& $\mathrm{C}$ & 0,938 & $-0,243$ \\
& $\mathrm{O}$ & $-1,145$ & 0,036 \\
\hline & $\mathrm{Zn}$ & 1,140 & \\
& $\mathrm{C}$ & 0,645 & 0,103 \\
& $\mathrm{~N}$ & $-1,430$ & 0,112 \\
\hline
\end{tabular}

${ }^{a}$ As cargas atômicas dos carbonos, oxigênios e nitrogênios são as médias desta propriedade para todos os átomos de cada tipo do complexo.

${ }^{\mathrm{b}} \Delta q=q($ complexo $)-q($ ligantes $)$. 


\section{CONCLUSÕES}

A maioria dos complexos metálicos estudados apresentam frequências de estiramento de ligantes menores quando comparadas às dos ligantes livres, exibindo, assim, um deslocamento das bandas $\mathrm{CO}$ e $\mathrm{CN}$ para comprimentos de onda maiores do espectro de infravermelho. Além disso, estas bandas vibracionais tornam-se, de modo geral, mais intensas com a complexação do ligante ao metal devido à contribuição cruzada entre a carga e o fluxo de carga $\left(\triangle A^{\mathrm{C} \times \mathrm{CF}}\right)$. Possivelmente, as variações de intensidade estariam associadas com a efetividade da retrodoação $\pi$ metal - ligante.

Em relação às variações das propriedades topológicas durante o processo de coordenação, observa-se, na maioria dos casos (exceto nos complexos com centros metálicos com NOX 2 ou 3), uma diminuição da densidade eletrônica no BCP da ligação CO quando esta molécula está ligada ao metal, indicando um enfraquecimento da ligação CO. Este enfraquecimento parece estar principalmente ligado à efetividade da retrodoação metal ligante. Além disso, a partição energética IQA da energia interatômica das ligações metalcarbono, carbono-oxigênio e carbono-nitrogênio mostra que a contribuição eletrostática (majoritariamente iônica) tende a ser, em geral, predominante em relação àquela de trocacorrelação (majoritariamente covalente). Além disto, a contribuição de troca-correlação para as ligações CO mostra um padrão de variações durante a complexação que parece reforçar as expectativas sobre a retrodoação nas carbonilas estudadas.

Por fim, as cargas atômicas QTAIM dos metais e dos carbonos são sempre positivas e as dos oxigênios e nitrogênios são sempre negativas. Estas cargas suportam um padrão onde a retrodoação metal - ligante é maior que a doação ligante - metal para complexos com centros metálicos apresentando NOX $=0$ e 1 . O oposto é observado para complexos com centros metálicos em que o NOX é igual ou maior que 2.

\section{PERSPECTIVAS}

Analisar as propriedades espectroscópicas, topológicas e energéticas de outros complexos contendo o ligante cianeto, isoeletrônico do $\mathrm{CO}$, a fim de comparar melhor com os resultados obtidos para as carbonilas metálicas.

\section{REFERÊNCIAS}

BADER, R. F. W. Atoms in Molecules: A Quantum Theory. $1^{\mathrm{a}}$ ed. Oxford, UK: Clarendon 
Press, 1990.

BERNHARDT, E.; BLEY, B.; WARTCHOW, R.; WILLNER, H.; BILL, E.; KUHN, P.; SHAM, I. H. T.; BODENBINDER, M.; BRÖCHLER, R. e AUBKE, F. Journal of the American Chemical Society. v. 121, p. 7188-7200,1999.

BLANCO, M. A.; PENDÁS, A. M. e FRANCISCO, E. Interacting quantum atoms: a correlated energy decomposition scheme based on the quantum theory of atoms in molecules. Journal of Chemical Theory and Computation, v. 1, p. 1096-1109, 2005.

CHATT, J. e DUNCANSON, L. A. Olefin co-ordination compounds. Part III. Infrared spectra and structure: attempted preparation of acetylene comlexes. Journal of the Chemical Society, v. 0, p.2622-2929, 1953.

CORTÉS-GUZMÁN, F e BADER, R. F. W. Complementary of QTAIM and MO theory in the study of bonding in donor-acceptor complexes. Coordination Chemistry Reviews, v. 249, p. 633-662, 2005.

CRABTREE, R. H. The organometallic chemistry of the transition metals. $4^{\mathrm{a}}$ ed. New Jersey, US: John Wiley \& Sons, 2005.

DAVIDE, T., FRANCISCO, E., BLANCO, M. A., MACHI, P., SIRONI, A. e PENDÁS, A. M. Bonding in classical and nonclassical transition metal carbonyls: the Interacting Quantum Atoms perspective. Journal of Chemical Theory and Computation, v. 6, p. 1064-1074, 2010.

DEWAR, M. A review of $\pi$ complex theory. Bulletin de la Societé Chimique de France, v. 18, C71-C79, 1951.

DUNNING JR, T. H. Gaussian basis sets for use in correlated molecular calculations. I. The atoms boron through neon and hydrogen. The Journal of Chemical Physics, v. 90, p. 10071023, 1989.

FARRUGIA, L. J. e EVANS, C. Experimental X-ray charge density studies studies on the binary carbonyls $\mathrm{Cr}(\mathrm{CO})_{6}, \mathrm{Fe}(\mathrm{CO})_{5}$, and $\mathrm{Ni}(\mathrm{CO})_{4}$. Journal of Chemical Physics A, v. 109, p. 8834-8848, 2005.

FAN, L. e ZIEGLER, T. Application of Density Functional Theory to infrared absorption intensity calculations on transition-metal carbonyls. The Journal of Physical Chemistry, v. 96, p. 6938-6941, 1992.

FRISCH, M. J.; TRUCKS G. W.; SCHLEGEL, H. B.; SCUSERIA, G. E.; ROBB, M. A.; CHEESEMAN, J. R.; SCALMANI, G.; BARONE, V.; MENNUCCI, B.; PETERSSON, G. A.; NAKATSUJI, H.; CARICATO, M.; LI, X.; HRATCHIAN, H. P.; IZMAYLOV, A. F.; BLOINO, J.; ZHENG, G.; SONNENBERG, J. L.; HADA, M.; EHARA, M.; TOYOTA, K.; FUKUDA, R.; HASEGAWA, J.; ISHIDA, M.; NAKAJIMA, T.; HONDA, Y.; KITAO, O.; NAKAI, H.; VREVEN, T.; MONTGOMERY JR., J. A.; PERALTA, J. E.; OGLIARO, F.; BEARPARK, M.; HEYD, J. J.; BROTHERS, E.; KUDIN, K. N.; STAROVEROV, V. N.; KEITH, T.; KOBAYASHI, R.; NORMAND, J.; RAGHAVACHARI, K.; RENDELL, A.; BURANT, J. C.; IYENGAR, S. S.; TOMASI, J.; COSSI, M.; REGA, N.; MILLAM, J. M.; 
KLENE, M.; KNOX, J. E.; CROSS, J. B.; BAKKEN, V.; ADAMO, C.; JARAMILLO, J.; GOMPERTS, R.; STRATMANN, R. E.; YAZYEV, O.; AUSTIN, A. J.; CAMMI, R.; POMELLI, C.; OCHTERSKI, J. W.; MARTIN, R. L.; MOROKUMA, K.; ZAKRZEWSKI, V. G.; VOTH, G. A.; SALVADOR, P.; DANNENBERG, J. J.; DAPPRICH, S.; DANIELS, A. D.; FARKAS, O.; FORESMAN, J. B.; ORTIZ, J. V.; CIOSLOWSKI, J. e FOX, D. J. Gaussian 09, Revision D.01, Gaussian Inc., Wallingford, CT, 2013.

GEIER, J.; WILLNER, H.; LEHMANN, C. W. e AUBKE, F. Formation of hexacarbonylmanaganese(I) salts, $\left[\mathrm{Mn}(\mathrm{CO})_{6}\right]^{+} \mathrm{X}^{-}$, in anhydrous HF. Inorganic Chemistry, v. 46, p. 7210-7214, 2007.

GRIMME, S. Semiempirical hybrid density functional with perturbative second-order correlation. The Journal of Chemical Physics, v. 124, 034108, 2006.

GOLDMAN, A. S. e KROGH-JESPERSEN, K. Why do cationic carbon monoxide complexes have high $\mathrm{C}-\mathrm{O}$ stretching force constants and short $\mathrm{C}-\mathrm{O}$ bonds? Electrostatic effects, not $\sigma$-bonding. Journal of the American Chemical Society. v. 118, p. 12159$12166,1996$.

HAIDUKE, R. L. A. e BRUNS, R. E. An atomic charge-charge flux-dipole flux atom-inmolecule decomposition for molecular dipole-moment derivatives and infrared fundamental intensities. Journal of Chemical Physics A, v. 109, p. 2680-2688, 2005.

HANSFORD, G. M. e DAVIES, P. B. Infrared laser spectroscopy of jet-cooled chromium hexacarbonyl in the 5- $\mu \mathrm{m}$ region. Journal of Molecular spectroscopy, v. 168, p. 540-555, 1994.

HAYNES, W. M. Ed. CRC Handbook of Chemistry e Physics; 96th ed. CRC Press, Taylor e Francis: Boca Raton, FL, 2016.

HOUSECROFT, C. E. e SHARPE, A. G. Inorganic chemistry. $3^{\text {a }}$ ed. Person: Prentice Hall, 2008. 807 p.

HUHEEY, J. E.; KEITER, E. A. e KEITER, R. L. Inorganic Chemistry Principles of Structure and Reactivity; Harper Collins: New York, 1993.

JONES, L. H.; McDOWELL, R. S. e GOLDBLATT, M. Potential Constants of Iron Pentacarbonyl from Vibrational Spectra of Isotopic Species. The Journal of Chemical Physics. v. 57, p. 2050-2064, 1972.

KENDALL, R. A.; DUNNING JR., T.H. e HARRISON, R.J. Electron affinities of the first-row atoms revisited. Systematic basis sets and wave functions. The Journal of Chemical Physics, v. 96, p. 6796, 1992.

LUPINETTI, A. J.; FRENKING, G. e STRAUSS, S. H. Nonclassical Metal Carbonyls. Angewandte Chemie International Edition. v. 37, p. 2113-2116, 1998.

NATIONAL INSTITUTE OF STANDARDS AND TECHNOLOGY. Chemistry webbook. U. S. Secretary of Commerce: the standard reference data act. 1968. Disponível em: <http//www.nist.gov/index.html>. Acesso em: 5 dez. 2017. 
PENDÁS, A. M.; BLANCO, M. A. e FRANCISCO, E. The nature of the hydrogen bond: a synthesis from the interacting quantum atoms picture. The Journal of Chemical Physics, $v$. 125, p. 184-112, 2006.

REED, Z. D e DUNCAN, M. A. Infrared spectroscopy and structures of manganese carbonyl cations, $\mathrm{Mn}(\mathrm{CO})_{\mathrm{n}}{ }^{+}(\mathrm{n}=1-9)$. Journal of the American Society for Mass Spectrometry, v. 21, p.739-749, 2010.

RICKS, A. M.; BAKKER, J. M.; DOUBERLY, G. E. e DUNCAN, M. A. Infrared spectroscopy and structures of cobalt carbonyl cations, $\operatorname{Co}(\mathrm{CO})_{\mathrm{n}}{ }^{+}(\mathrm{n}=1-9)$. Journal of Chemical Physics A, v. 113, p. 4701-4708, 2009.

SCHAFTENAAR, G. e NOORDIK J. H. Molden: a pre- and post-processing program for molecular and electronic structures, Journal Computational - Aided Molecular Design, v. 14, p. 123-134, 2000.

WILSON, A. K.; WOON, D. E.; PETERSON, K. A. e DUNNING JR, T. H. Gaussian basis sets for use in correlated molecular calculations. IX. The atoms gallium through krypton. Journal of Chemical Physics, v. 110, 7667-7676, 1999.

WOON, D. E. e DUNNING Jr., T. H. Gaussian-basis sets for use in correlated molecular calculations. 3. The atoms aluminum through argon. The Journal of Chemical Physics, v. 98, p. $1358-1371,1993$.

WRIGHTON, M. The Photochemistry of Metal Carbonyls. Chemical Review, v. 74, p. 401430, 1974. 
Capítulo 4

Análise das variações das intensidades de infravermelho durante a excitação eletrônica segundo o modelo de Carga - Fluxo de Carga Fluxo de Dipolo 


\section{INTRODUÇÃO}

Tradicionalmente, a Teoria Quântica de Átomos e Moléculas, utilizada para a partição Carga - Fluxo de Carga - Fluxo de Dipolo (CCFDF), é concebida como uma "teoria de moléculas no estado fundamental" para o cálculo de cargas e dipolos atômicos de diversos sistemas, como os descritos anteriormente neste trabalho (BADER, 1990; HAIDUKE e BRUNS, 2005; MATTA e BOYD, 2007; FERRO-COSTAS et al., 2014; TERRABUIO et al., 2014). Assim, a teoria proposta por Bader não é usualmente empregada para compreender fenômenos fotofísicos e fotoquímicos que envolvam estados excitados (FERRO-COSTAS et al., 2014). Um processo fotoquímico envolve a transição de um elétron do estado fundamental para um estado excitado devido à absorção de luz por uma molécula. Esta transição eletrônica, que poderia ocorrer do orbital molecular ocupado de mais alta energia (HOMO) para o orbital molecular não ocupado de menor energia (LUMO), resulta em uma nova espécie química com características e propriedades diferentes daquela no estado fundamental (ADAMSON, 1983). Durante a excitação eletrônica, um estado singleto ou tripleto podem ser produzidos (na verdade são obtidos três estados tripletos degenerados e um estado singleto neste processo). Se os spins permanecem emparelhados no estado excitado, a molécula está no estado singleto e, se os spins tornam-se desemparelhados, a molécula está em um estado excitado tripleto, como ilustrado na Figura 16.

Figura 16 - Representação esquemática dos estados de spin eletrônico singleto e tripleto durante uma excitação hipotética do tipo HOMO - LUMO

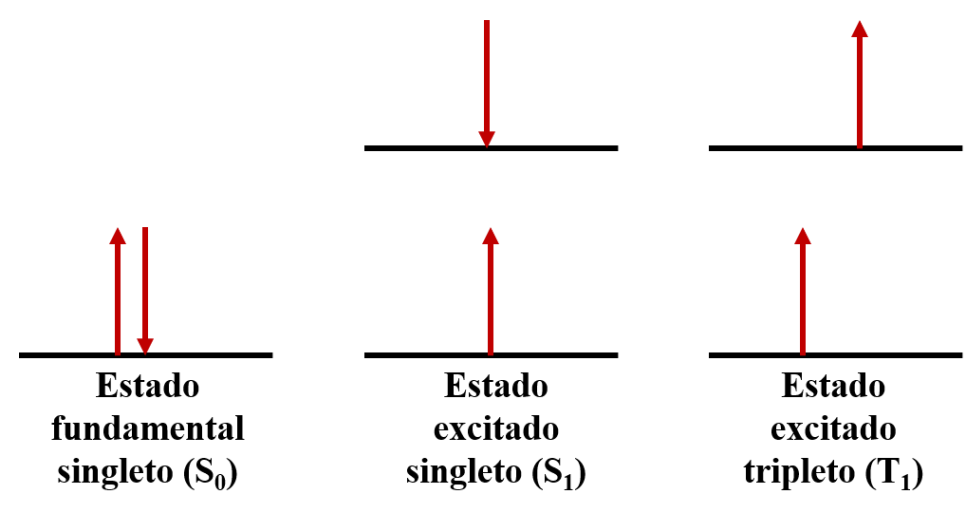

O tempo de vida de uma espécie excitada é breve porque há diversos mecanismos pelos quais ela pode perder o seu excesso de energia e decair novamente para o estado fundamental. Esta perda de energia pode ocorrer por conta das colisões entre as moléculas 
excitadas e as moléculas de solvente, resultando na desativação vibracional ou em virtude da relaxação não radiativa entre os níveis vibracionais mais baixos de um estado eletrônico e os níveis vibracionais mais altos de outro estado eletrônico (conversão interna), como mostrado na Figura 17 (DEMAS, 1983; LAKOWICZ, 2010). O estado excitado também pode ser desativado por meio de processos radiativos como a fluorescência e a fosforescência (DEMAS, 1983). A fluorescência em moléculas ocorre a partir do estado excitado com multiplicidade de spin igual àquela do estado fundamental (DEMAS, 1983; LAKOWICZ, 2010). Esta transição é altamente provável e o tempo de vida do estado excitado é curto. Por sua vez, a fosforescência envolve a transição a partir de um estado excitado com multiplicidade diferente do estado fundamental (LAKOWICZ, 2010). Como há uma alteração do spin eletrônico neste último caso, a transição é menos provável e, portanto, o tempo de vida do estado excitado é mais longo.

Figura 17 - Diagrama de Jablonski ilustrando um processo fotoquímico

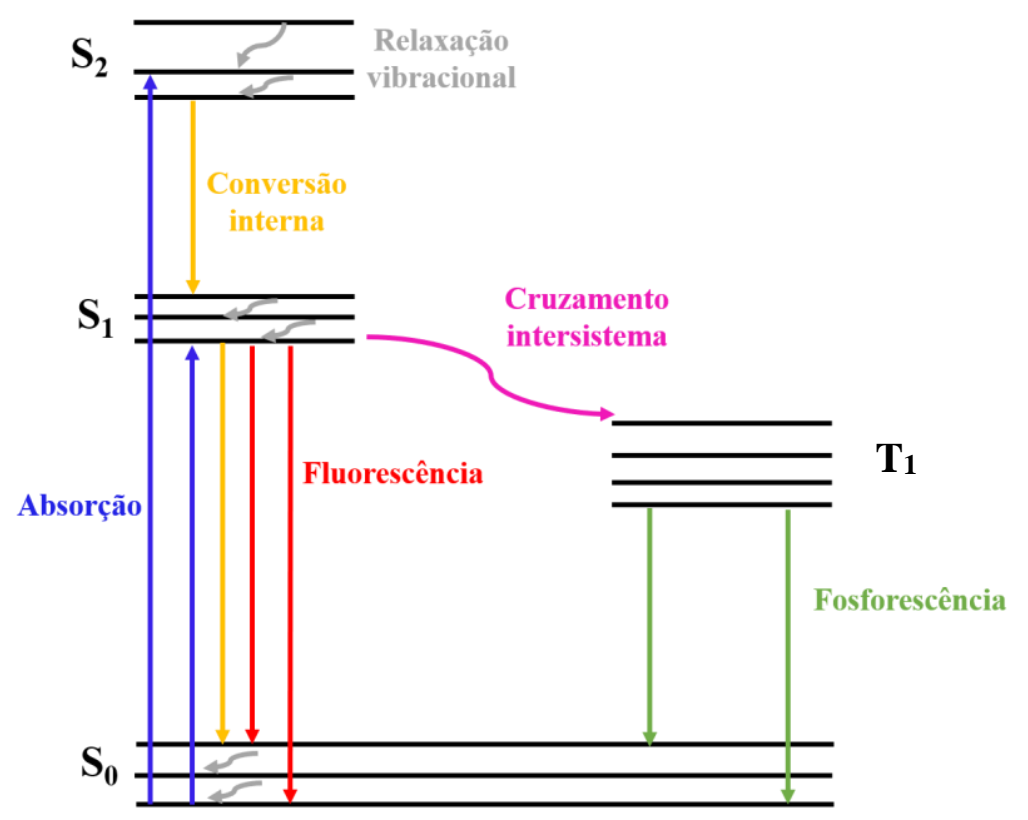

Fonte: Adaptado de LAKOWICZ, J. R. Principles of Fluorescence Spectroscopy, $3^{\mathrm{a}}$ ed., Springer: USA, 2010.

A descrição da superfície de energia potencial de estados excitados é mais complexa quando comparada a do estado fundamental. De modo geral, estas superfícies envolvem muitos mínimos locais, estados de transição, cruzamentos intersistemas e estados acoplados (SERRANO-ANDRÉS e MERCHÁN, 2005; GARCÍA-REVILLA et al., 2018). Além disto, existem muitos tipos de estados excitados que requerem quantidades distintas de correlação 
eletrônica e conjuntos de funções de base apropriadas que abarquem os efeitos inerentes a estes estados simultaneamente (SERRANO-ANDRÉS e MERCHÁN, 2005). Outro problema associado ao estudo da estrutura eletrônica destes estados é a invalidação da aproximação Born-Oppenheimer quando eles estão muito próximos energeticamente (GARCÍA-REVILLA et al., 2018). Tais dificuldades resultam em métodos e algoritmos não tão eficientes quanto aqueles usados para os tratamentos teóricos dos estados fundamentais (SERRANO-ANDRÉS e MERCHÁN, 2005).

Assim, apesar de haver grande quantidade de estudos para o estado fundamental de átomos, moléculas e sólidos por meio da Teoria Quântica de Átomos em Moléculas (QTAIM), não há uma contrapartida tão ampla no que se refere aos estados excitados eletrônicos. Algumas investigações sobre a topologia da densidade eletrônica durante processos de excitação incluem análises de interações que não são covalentes (JARACORTÉS, ROCHA-RINZA e HERNÁDEZ-TRUJILLO, 2015), reações de foto-isomerização (GUTIERREZ-ARZALUZ, ROCHA-RINZA e CORTÉZ-GÚZMAN, 2015) e estudos sobre a teoria do orbital molecular durante rearranjos eletrônicos (FERRO-COSTAS et al., 2014). A combinação da QTAIM com a Teoria do Funcional de densidade dependente do tempo (TDDFT) tem propiciado diversos avanços na compreensão de fenômenos fotofísicos e fotoquímicos (GARCÍA-REVILLA et al., 2018). A TD-DFT é baseada na solução da equação da resposta linear durante uma perturbação radiativa para obter a energia de excitação e o momento de transição, evitando, assim, o cálculo explícito do estado excitado considerado (SERRANO-ANDRÉS e MERCHÁN, 2005). As energias de excitação podem ser verticais ou adiabáticas. No primeiro caso, as energias dos estados excitados são calculadas na geometria de equilíbrio do estado fundamental, enquanto no segundo, esta propriedade é obtida na geometria de equilíbrio do próprio estado, como ilustra a Figura 18 (COYLE, 2004). 
Figura 18 - Energias de transição vertical e adiabática

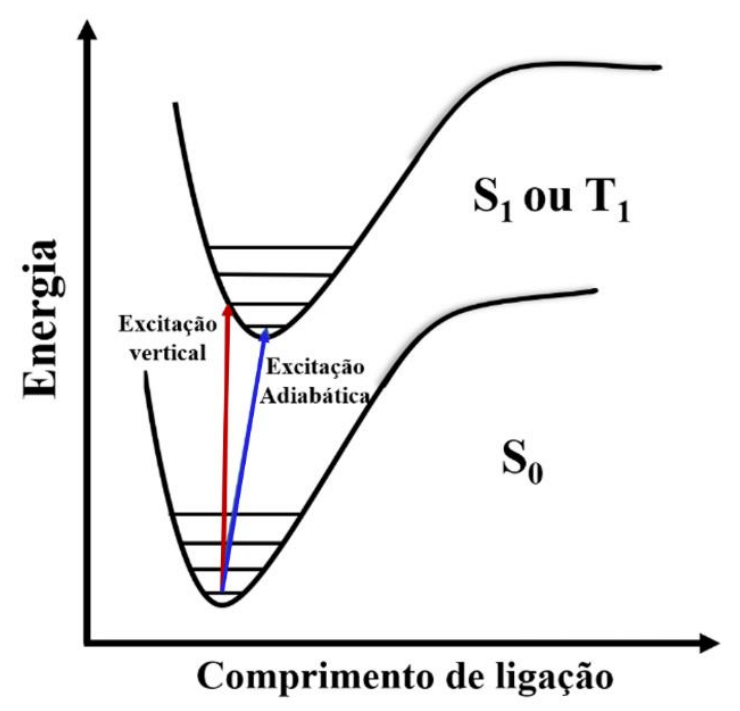

Compreender a natureza dos estados excitados apresenta grande relevância para áreas do conhecimento que abrangem desde a síntese orgânica e a química inorgânica até a nanotecnologia e a biologia e uma ferramenta útil para esta análise é a espectroscopia de infravermelho. Neste contexto, desde a formalização do modelo CCFDF/QTAIM, apenas moléculas nos estados fundamentais haviam sido submetidas a uma análise por meio desta partição. Neste sentido, o estudo da distribuição eletrônica em estados excitados e de suas variações durante modos vibracionais representa um campo de pesquisa com amplas oportunidades para a aplicação deste modelo. O primeiro estudo das variações das intensidades de infravermelho durante a excitação eletrônica segundo o modelo CCFDF/QTAIM foi realizado para a molécula de monóxido de carbono (CO) por nosso grupo de pesquisa (TERRABUIO et al., 2017). Neste trabalho, foi mostrado que o enfraquecimento da banda de estiramento CO durante a excitação para os estados singleto e tripleto de menor energia é devido, principalmente, a mudanças nas cargas atômicas. Em seguida, Martins e De Oliveira (2017) aplicaram o mesmo modelo de partição para os estados fundamental e excitados da molécula de ozônio. Para este sistema, as mudanças nas intensidades de infravermelho podem ser interpretadas como resultado das contribuições de carga e de fluxo de carga.

As alterações de propriedades moleculares durante a excitação eletrônica refletem na reatividade química de tais compostos. Um exemplo é o momento de dipolo, que pode apresentar até mesmo inversões de sentido (TERRABUIO et al., 2017). Neste contexto, algumas moléculas diatômicas simples, como hidreto, monofluoreto, monocloreto e monobrometo de alumínio ( $\mathrm{AlH}, \mathrm{AlF}, \mathrm{AlCl}$ e $\mathrm{AlBr}$, respectivamente), monofluoreto, 
monocloreto e monobrometo de boro (BF, $\mathrm{BCl}$ e $\mathrm{BBr}$ ) e monossulfeto de carbono (CS), foram escolhidas para analisar como a QTAIM explica as mudanças de propriedades moleculares devido a excitação eletrônica e como a partição CCFDF/QTAIM explica as variações das intensidades de infravermelho durante este processo. Para isso, três estados eletrônicos foram considerados no estudo de cada caso, o estado fundamental $\left(\mathrm{S}_{\mathrm{o}}\right)$ e os primeiros estados excitados singleto $\left(\mathrm{S}_{1}\right)$ e tripleto $\left(\mathrm{T}_{1}\right)$ de menor energia.

\section{OBJETIVOS}

2.1 Avaliar os efeitos da excitação eletrônica em propriedades, como distâncias de ligação, momentos de dipolo moleculares, multipolos atômicos, frequências vibracionais e intensidades de infravermelho durante a excitação eletrônica das moléculas diatômicas simples escolhidas;

2.2 Analisar as mudanças nos componentes de energia intra- e interatômica durante a excitação eletrônica segundo a Partição Virial;

2.3 Aplicar o modelo de partição CCFDF/QTAIM para decompor as variações nas intensidades de infravermelho dos estados excitados tripleto e singleto de menor energia dos sistemas considerados em comparação ao estado fundamental.

\section{METODOLOGIA}

O estudo dos estados excitados das moléculas diatômicas hidreto, monofluoreto, monocloreto e monobrometo de alumínio ( $\mathrm{AlH}, \mathrm{AlF}, \mathrm{AlCl}$ e $\mathrm{AlBr}$ ), monofluoreto, monocloreto e monobrometo de boro ( $\mathrm{BF}, \mathrm{BCl}$ e $\mathrm{BBr}$ ) e monossulfeto de carbono (CS), foi realizado no programa Gaussian 09 (FRISCH et al., 2013). Para isso, o funcional híbrido de troca e correlação PBE0, que tem sido bem-sucedido na determinação das propriedades de tais estados (JACQUEMIN et al., 2009; LEANG, ZAHARIEV e GORDON, 2012), e o conjunto de base triplo- $\zeta$ de Dunning (1989) contendo funções difusas, aug-cc-pVTZ, as quais são indicadas para descrever o caráter difuso da densidade eletrônica dos estados excitados, foram selecionados (KENDALL, DUNNING JR. e HARRISON, 1992; WILSON et al., 1999; WOON e DUNNING JR, 1993). O estado fundamental foi calculado utilizando a versão restrita da Teoria do Funcional de Densidade (DFT), enquanto a versão dependente do tempo 
da DFT (TD-DFT) forneceu os resultados para os estados excitados, conforme as suas implementações no programa Gaussian 09 (FRISCH et al., 2013).

Os estados fundamentais e excitados tiveram suas distâncias de ligação otimizadas utilizando um critério de convergência mais rigoroso, mediante a palavra-chave "verytight". Uma grade de pontos mais acurada também foi utilizada em integrais avaliadas de forma numérica (palavra-chave "ultrafine"). A partir destas estruturas correspondentes ao ponto de mínimo da superfície de energia potencial, foram obtidas as energias de excitação, as cargas e dipolos atômicos, além das frequências e intensidades vibracionais. Por sua vez, avaliou-se o efeito da excitação nos componentes inter- e intra-atômicos da energia eletrônica por meio de uma análise baseada nas partições QTAIM e segundo o teorema Virial. Por fim, as contribuições de carga, fluxo de carga e fluxo de dipolo para as intensidades de infravermelho foram obtidas via o modelo CCFDF/QTAIM, conforme o procedimento já descrito para os dímeros bidentados e as carbonilas metálicas nos capítulos anteriores.

\section{RESULTADOS E DISCUSSÕES}

\subsection{Energias de excitação vertical e adiabática}

A Tabela 20 contém as energias de transição verticais e adiabáticas do estado singleto fundamental $\left(\mathrm{S}_{0}\right)$ e para os estados excitados tripleto $\left(\mathrm{T}_{1}\right)$ e singleto $\left(\mathrm{S}_{1}\right)$ de menor energia das moléculas de $\mathrm{AlH}, \mathrm{AlF}, \mathrm{AlCl}, \mathrm{AlBr}, \mathrm{BF}, \mathrm{BCl}, \mathrm{BBr}$ e $\mathrm{CS}$, as quais foram obtidas a partir de cálculos PBE0/aug-cc-pVTZ. Os respectivos dados experimentais disponíveis também foram incluídos na mesma tabela. Os resultados deste nível de cálculo concordam satisfatoriamente com os dados experimentais encontrados, sendo que os maiores desvios ocorrem para os estados tripletos dos sistemas $\mathrm{BF}, \mathrm{BCl}$ e $\mathrm{BBr}(0,89$ a $0,93 \mathrm{eV})$. Em todos os casos, o tratamento teórico empregado para esta análise subestima os valores de energias de excitação verticais e adiabáticas em relação aos dados experimentais disponíveis.

Tabela 20 - Energias de excitação verticais $\left(\Delta E_{v e r t}\right)$ e adiabáticas $\left(\Delta E_{a d}\right)$ das moléculas $\mathrm{AlH}$, $\mathrm{AlF}, \mathrm{AlCl}, \mathrm{AlBr}, \mathrm{BF}, \mathrm{BCl}, \mathrm{BBr}$ e CS, calculadas com o nível de teoria PBE0/aug-cc-pVTZ $(\mathrm{em} \mathrm{eV})$

\begin{tabular}{|c|c|c|c|c|c|}
\hline & \multicolumn{2}{|l|}{ Vertical } & \multicolumn{3}{|c|}{ Adiabático } \\
\hline Transição & $\Delta E_{\text {vert(raw) }}{ }^{\mathrm{a}}$ & $\Delta E_{a d(\text { raw })}{ }^{\mathrm{a}}$ & Desv. $^{\mathrm{c}}$ & $\Delta E_{a d}^{\mathrm{b}}$ & Desv. $^{c}$ \\
\hline $\mathrm{AlH}$ & & & & & \\
\hline$a^{3} \Pi \leftarrow X^{1} \Sigma^{+}$ & 1,31 & 1,27 & & 1,28 & \\
\hline
\end{tabular}




\begin{tabular}{|c|c|c|c|c|c|}
\hline & Vertical & & Adiabá & ítico & \\
\hline Transição & $\Delta E_{\text {vert(raw) }}{ }^{\text {a }}$ & $\Delta E_{a d(\text { raw })^{\mathrm{a}}}$ & Desv. ${ }^{\mathrm{c}}$ & $\Delta E_{a d}^{\mathrm{b}}$ & Desv. $^{\mathrm{c}}$ \\
\hline $\mathrm{A}^{1} \Pi \leftarrow \mathrm{X}^{1} \Sigma^{+}$ & 2,86 & 2,86 & $-0,05$ & 2,84 & \\
\hline Exp. & & 2,91 & & & \\
\hline \multicolumn{6}{|l|}{$\mathrm{AlF}$} \\
\hline$a^{3} \Pi \leftarrow X^{1} \Sigma^{+}$ & 2,77 & 2,77 & $-0,61$ & 2,77 & $-0,61$ \\
\hline Exp. & & 3,38 & & 3,38 & \\
\hline $\mathrm{A}^{1} \Pi \leftarrow \mathrm{X}^{1} \Sigma^{+}$ & 5,32 & 5,32 & $-0,13$ & 5,32 & $-0,13$ \\
\hline Exp. & & 5,45 & & 5,45 & \\
\hline \multicolumn{6}{|l|}{$\mathrm{AlCl}$} \\
\hline$a^{3} \Pi \leftarrow X^{1} \Sigma^{+}$ & 2,47 & 2,46 & $-0,58$ & 2,46 & $-0,58$ \\
\hline Exp. & & 3,04 & & 3,04 & \\
\hline $\mathrm{A}^{1} \Pi \leftarrow \mathrm{X}^{1} \Sigma^{+}$ & 4,64 & 4,64 & $-0,10$ & 4,64 & $-0,10$ \\
\hline Exp. & & 4,74 & & 4,74 & \\
\hline \multicolumn{6}{|l|}{$\mathrm{AlBr}$} \\
\hline$a^{3} \Pi \leftarrow X^{1} \Sigma^{+}$ & 2,39 & 2,37 & $-0,56$ & 2,38 & $-0,56$ \\
\hline Exp. & & 2,93 & & 2,93 & \\
\hline $\mathrm{A}^{1} \Pi \leftarrow \mathrm{X}^{1} \Sigma^{+}$ & 4,35 & 4,35 & $-0,10$ & 4,34 & $-0,11$ \\
\hline Exp. & & 4,44 & & 4,45 & \\
\hline \multicolumn{6}{|l|}{$\mathrm{BF}$} \\
\hline $\mathrm{a}^{3} \Pi \leftarrow \mathrm{X}^{1} \Sigma^{+}$ & 2,79 & 2,72 & $-0,89$ & 2,71 & $-0,90$ \\
\hline Exp. & & 3,61 & & 3,61 & \\
\hline $\mathrm{A}^{1} \Pi \leftarrow \mathrm{X}^{1} \Sigma^{+}$ & 6,16 & 6,13 & $-0,20$ & 6,13 & $-0,22$ \\
\hline Exp. & & 6,33 & & 6,34 & \\
\hline \multicolumn{6}{|l|}{$\mathrm{BCl}$} \\
\hline$a^{3} \Pi \leftarrow X^{1} \Sigma^{+}$ & 1,59 & 1,58 & $-0,93$ & 1,59 & $-0,92$ \\
\hline Exp. & & 2,51 & & 2,50 & \\
\hline $\mathrm{A}^{1} \Pi \leftarrow \mathrm{X}^{1} \Sigma^{+}$ & 4,40 & 4,39 & & 4,39 & $-0,17$ \\
\hline Exp. & & 4,56 & $-0,17$ & 4,56 & \\
\hline \multicolumn{6}{|l|}{$\mathrm{BBr}$} \\
\hline $\mathrm{a}^{3} \Pi \leftarrow \mathrm{X}^{1} \Sigma^{+}$ & 1,44 & 1,41 & $-0,91$ & 1,42 & $-0,90$ \\
\hline Exp. & & 2,32 & & 2,32 & \\
\hline $\mathrm{A}^{1} \Pi \leftarrow \mathrm{X}{ }^{1} \Sigma^{+}$ & 4,04 & 4,02 & $-0,19$ & 4,02 & $-0,19$ \\
\hline Exp. & & 4,20 & & 4,21 & \\
\hline \multicolumn{6}{|l|}{ CS } \\
\hline$a^{3} \Pi \leftarrow X^{1} \Sigma^{+}$ & 2,97 & 2,95 & $-0,47$ & 2,94 & $-0,49$ \\
\hline Exp. & & 3,42 & & 3,43 & \\
\hline $\mathrm{A}^{1} \Pi \leftarrow \mathrm{X}^{1} \Sigma^{+}$ & 4,79 & 4,76 & $-0,05$ & 4,75 & $-0,08$ \\
\hline Exp. & & 4,81 & & 4,82 & \\
\hline
\end{tabular}

${ }^{a}$ Energias não corrigidas pelas energias do ponto zero.

${ }^{\mathrm{b}}$ Energias corrigidas pelas energias do ponto zero.

${ }^{\mathrm{c}}$ Desv. $=\Delta E$ (teórico) $-\Delta E($ exp.).

d NATIONAL INSTITUTE OF STANDARDS E TECHNOLOGY. Chemistry webbook. U. S. Secretary of Commerce: the standard reference data act. 1968. Disponível em: <http//www.nist.gov/index.html>. Acesso em: 
05 dez. 2017.

\subsection{Energias atômicas obtidas a partir da aproximação Virial}

A Tabela 21 mostra a decomposição da energia eletrônica segundo a Partição Virial em contribuições intra- e interatômicas, obtidas em cálculos PBE0/aug-cc-pVTZ, para os estados fundamentais e excitados adiabaticamente dos sistemas estudados.

Tabela 21 - Componentes da energia atômica, segundo a Partição Virial, obtidas por meio de cálculos PBE0/aug-cc-pVTZ nas geometrias de equilíbrio de cada estado (em u. a.)

\begin{tabular}{|c|c|c|c|c|c|c|c|c|c|}
\hline \multirow{2}{*}{$\frac{\text { Sistema/Estado }}{\mathrm{AlH}}$} & \multirow[t]{2}{*}{ Prop. } & \multicolumn{2}{|c|}{ Átomos } & \multirow[t]{2}{*}{ Soma } & \multirow{2}{*}{$\frac{\text { Sistema/Estado }}{\mathrm{BF}}$} & \multirow[t]{2}{*}{ Prop. } & \multicolumn{2}{|c|}{ Átomos } & \multirow[t]{2}{*}{ Soma } \\
\hline & & $\mathrm{Al}$ & $\mathrm{H}$ & & & & B & $\mathrm{F}$ & \\
\hline \multirow[t]{3}{*}{$\mathrm{X}^{1} \Sigma^{+}$} & $E_{\text {Intra }}$ & $-241,599$ & $-0,392$ & $-241,991$ & $\mathrm{X}^{1} \Sigma^{+}$ & $E_{\text {Intra }}$ & $-24,057$ & $-99,190$ & $-123,247$ \\
\hline & $E_{\text {Inter }}$ & 0,134 & $-0,328$ & $-0,195$ & & $E_{\text {Inter }}$ & $-0,031$ & $-0,676$ & $-0,707$ \\
\hline & $E^{a}$ & $-241,465$ & $-0,720$ & $-242,186$ & & $E^{a}$ & $-24,088$ & $-99,866$ & $-123,954$ \\
\hline \multirow[t]{6}{*}{$a^{3} \Pi$} & $E_{\text {Intra }}$ & $-241,577$ & $-0,394$ & $-241,971$ & $a^{3} \Pi$ & $E_{\text {Intra }}$ & $-24,047$ & $-99,217$ & $-123,265$ \\
\hline & $E_{\text {Inter }}$ & 0,089 & $-0,283$ & $-0,194$ & & $E_{\text {Inter }}$ & $-0,052$ & $-0,549$ & $-0,601$ \\
\hline & $E^{a}$ & $-241,488$ & $-0,677$ & $-242,165$ & & $E^{a}$ & $-24,099$ & $-99,766$ & $-123,866$ \\
\hline & $\Delta E_{\text {Intra }}{ }^{b}$ & 0,022 & $-0,002$ & 0,020 & & $\Delta E_{\text {Intra }}{ }^{b}$ & 0,010 & $-0,027$ & $-0,017$ \\
\hline & $\Delta E_{\text {Inter }}^{b}$ & $-0,044$ & 0,045 & 0,001 & & $\Delta E_{\text {Inter }}^{b}$ & $-0,022$ & 0,127 & 0,105 \\
\hline & $\Delta E^{b}$ & $-0,022$ & 0,043 & 0,021 & & $\Delta E^{b}$ & $-0,012$ & 0,100 & 0,088 \\
\hline \multirow[t]{6}{*}{$\mathrm{A}^{1} \Pi$} & $E_{\text {Intra }}$ & $-241,688$ & $-0,399$ & $-242,086$ & $\mathrm{~A}^{1} \Pi$ & $E_{\text {Intra }}$ & $-24,014$ & $-99,165$ & $-123,180$ \\
\hline & $E_{\text {Inter }}$ & 0,208 & $-0,211$ & $-0,003$ & & $E_{\text {Inter }}$ & 0,058 & $-0,608$ & $-0,550$ \\
\hline & $E^{a}$ & $-241,480$ & $-0,610$ & $-242,090$ & & $E^{a}$ & $-23,957$ & $-99,773$ & $-123,730$ \\
\hline & $\Delta E_{\text {Intra }}^{b}$ & $-0,089$ & $-0,007$ & $-0,096$ & & $\Delta E_{\text {Intra }}{ }^{b}$ & 0,043 & 0,025 & 0,068 \\
\hline & $\Delta E_{\text {Inter }}^{b}$ & 0,074 & 0,117 & 0,191 & & $\Delta E_{\text {Inter }}^{b}$ & 0,088 & 0,069 & 0,157 \\
\hline & $\Delta E^{b}$ & $-0,015$ & 0,110 & 0,096 & & $\Delta E^{b}$ & 0,131 & 0,093 & 0,224 \\
\hline $\mathrm{AlF}$ & & $\mathrm{Al}$ & $\mathrm{F}$ & & $\mathrm{BCl}$ & & B & $\mathrm{Cl}$ & \\
\hline \multirow[t]{3}{*}{$\mathrm{X}^{1} \Sigma^{+}$} & $E_{\text {Intra }}$ & $-241,540$ & $-99,335$ & $-340,875$ & $\mathrm{X}^{1} \Sigma^{+}$ & $E_{\text {Intra }}$ & $-24,145$ & $-459,308$ & $-483,454$ \\
\hline & $E_{\text {Inter }}$ & 0,116 & $-0,294$ & $-0,178$ & & $E_{\text {Inter }}$ & $-0,003$ & $-0,122$ & $-0,125$ \\
\hline & $E^{a}$ & $-241,424$ & $-99,629$ & $-341,053$ & & $E^{a}$ & $-24,148$ & $-459,430$ & $-483,578$ \\
\hline \multirow[t]{6}{*}{$a^{3} \Pi$} & $E_{\text {Intra }}$ & $-241,458$ & $-99,338$ & $-340,796$ & $a^{3} \Pi$ & $E_{\text {Intra }}$ & $-24,128$ & $-459,342$ & $-483,470$ \\
\hline & $E_{\text {Inter }}$ & 0,099 & $-0,277$ & $-0,178$ & & $E_{\text {Inter }}$ & $-0,070$ & 0,012 & $-0,057$ \\
\hline & $E^{a}$ & $-241,359$ & $-99,615$ & $-340,974$ & & $E^{a}$ & $-24,198$ & $-459,330$ & $-483,528$ \\
\hline & $\Delta E_{\text {Intra }}^{b}$ & 0,082 & $-0,003$ & 0,078 & & $\Delta E_{\text {Intra }}{ }^{b}$ & 0,017 & $-0,034$ & $-0,017$ \\
\hline & $\Delta E_{\text {Inter }}^{b}$ & $-0,016$ & 0,017 & 0,000 & & $\Delta E_{\text {Inter }}^{b}$ & $-0,067$ & 0,134 & 0,067 \\
\hline & $\Delta E^{b}$ & 0,065 & 0,014 & 0,079 & & $\Delta E^{b}$ & $-0,050$ & 0,100 & 0,050 \\
\hline
\end{tabular}




\begin{tabular}{|c|c|c|c|c|c|c|c|c|c|}
\hline \multirow{2}{*}{$\frac{\text { Sistema/Estado }}{\mathrm{A}^{1} \Pi}$} & \multirow{2}{*}{$\begin{array}{l}\text { Prop. } \\
\text { Entra }\end{array}$} & \multicolumn{2}{|c|}{ Átomos } & \multirow{2}{*}{$\begin{array}{l}\text { Soma } \\
-340,788\end{array}$} & \multirow{2}{*}{$\frac{\text { Sistema/Estado }}{\mathrm{A}^{1} \Pi}$} & \multirow{2}{*}{$\begin{array}{l}\text { Prop. } \\
E_{\text {Intra }}\end{array}$} & \multicolumn{2}{|c|}{ Átomos } & \multirow{2}{*}{$\begin{array}{c}\text { Soma } \\
-483,433\end{array}$} \\
\hline & & $-241,470$ & $-99,319$ & & & & $-24,163$ & $-459,270$ & \\
\hline & $E_{\text {Inter }}$ & 0,199 & $-0,278$ & $-0,079$ & & $E_{\text {Inter }}$ & 0,041 & $-0,012$ & 0,029 \\
\hline & $E^{a}$ & $-241,270$ & $-99,597$ & $-340,867$ & & $E^{a}$ & $-24,122$ & $-459,282$ & $-483,404$ \\
\hline & $\Delta E_{\text {Intra }}^{b}$ & 0,070 & 0,016 & 0,086 & & $\Delta E_{\text {Intra }}^{b}$ & $-0,017$ & 0,038 & 0,021 \\
\hline & $\Delta E_{\text {Inter }}^{b}$ & 0,084 & 0,016 & 0,099 & & $\Delta E_{\text {Inter }}^{b}$ & 0,043 & 0,110 & 0,153 \\
\hline & $\Delta E^{b}$ & 0,153 & 0,032 & 0,185 & & $\Delta E^{b}$ & 0,026 & 0,148 & 0,174 \\
\hline $\mathrm{AlCl}$ & & $\mathrm{Al}$ & $\mathrm{Cl}$ & & $\mathrm{BBr}$ & & B & $\mathrm{Br}$ & \\
\hline \multirow[t]{3}{*}{$\mathrm{X}^{1} \Sigma^{+}$} & $E_{\text {Intra }}$ & $-241,588$ & $-459,444$ & $-701,032$ & $\mathrm{X}^{1} \Sigma^{+}$ & $E_{\text {Intra }}$ & $-24,210$ & $-2572,308$ & $-2596,518$ \\
\hline & $E_{\text {Inter }}$ & 0,149 & 0,174 & 0,323 & & $E_{\text {Inter }}$ & 0,011 & 1,192 & 1,204 \\
\hline & $E^{a}$ & $-241,439$ & $-459,270$ & $-700,709$ & & $E^{a}$ & $-24,198$ & $-2571,115$ & $-2595,314$ \\
\hline \multirow[t]{6}{*}{$a^{3} \Pi$} & $E_{\text {Intra }}$ & $-241,510$ & $-459,433$ & $-700,943$ & $a^{3} \Pi$ & $E_{\text {Intra }}$ & $-24,182$ & $-2572,337$ & $-2596,519$ \\
\hline & $E_{\text {Inter }}$ & 0,115 & 0,188 & 0,303 & & $E_{\text {Inter }}$ & $-0,059$ & 1,303 & 1,244 \\
\hline & $E^{a}$ & $-241,395$ & $-459,245$ & $-700,640$ & & $E^{a}$ & $-24,241$ & $-2571,034$ & $-2595,275$ \\
\hline & $\Delta E_{\text {Intra }}{ }^{b}$ & 0,078 & 0,010 & 0,089 & & $\Delta E_{\text {Intra }}{ }^{b}$ & 0,028 & $-0,029$ & $-0,001$ \\
\hline & $\Delta E_{\text {Inter }}^{b}$ & $-0,034$ & 0,014 & $-0,019$ & & $\Delta E_{\text {Inter }}^{b}$ & $-0,070$ & 0,110 & 0,040 \\
\hline & $\Delta E^{b}$ & 0,045 & 0,025 & 0,070 & & $\Delta E^{b}$ & $-0,042$ & 0,081 & 0,039 \\
\hline \multirow[t]{6}{*}{$\mathrm{A}^{1} \Pi$} & $E_{\text {Intra }}$ & $-241,579$ & $-459,407$ & $-700,986$ & & & & & \\
\hline & $E_{\text {Inter }}$ & 0,203 & 0,239 & 0,443 & & & & & \\
\hline & $E^{a}$ & $-241,376$ & $-459,167$ & $-700,543$ & & & & & \\
\hline & $\Delta E_{\text {Intra }}^{b}$ & 0,009 & 0,037 & 0,046 & & & & & \\
\hline & $\Delta E_{\text {Inter }}^{b}$ & 0,055 & 0,066 & 0,120 & & & & & \\
\hline & $\Delta E^{b}$ & 0,063 & 0,103 & 0,166 & & & & & \\
\hline $\mathrm{AlBr}$ & & $\mathrm{Al}$ & $\mathrm{Br}$ & & $\mathrm{CS}$ & & $\mathrm{C}$ & $\mathrm{S}$ & \\
\hline \multirow[t]{3}{*}{$\mathrm{X}^{1} \Sigma^{+}$} & $E_{\text {Intra }}$ & $-241,607$ & $-2572,404$ & $-2814,011$ & $\mathrm{X}^{1} \Sigma^{+}$ & $E_{\text {Intra }}$ & $-37,281$ & $-396,985$ & $-434,265$ \\
\hline & $E_{\text {Inter }}$ & 0,158 & 1,368 & 1,526 & & $E_{\text {Inter }}$ & $-0,735$ & 0,160 & $-0,575$ \\
\hline & $E^{a}$ & $-241,449$ & $-2571,036$ & $-2812,485$ & & $E^{a}$ & $-38,015$ & $-396,825$ & $-434,840$ \\
\hline \multirow[t]{6}{*}{$a^{3} \Pi$} & $E_{\text {Intra }}$ & $-241,537$ & $-2572,393$ & $-2813,930$ & $a^{3} \Pi$ & $E_{\text {Intra }}$ & $-37,337$ & $-397,101$ & $-434,438$ \\
\hline & $E_{\text {Inter }}$ & 0,125 & 1,385 & 1,510 & & $E_{\text {Inter }}$ & $-0,597$ & 0,315 & $-0,282$ \\
\hline & $E^{a}$ & $-241,412$ & $-2571,008$ & $-2812,420$ & & $E^{a}$ & $-37,934$ & $-396,786$ & $-434,720$ \\
\hline & $\Delta E_{\text {Intra }}{ }^{b}$ & 0,071 & 0,011 & 0,082 & & $\Delta E_{\text {Intra }}{ }^{b}$ & $-0,056$ & $-0,117$ & $-0,173$ \\
\hline & $\Delta E_{\text {Inter }}^{b}$ & $-0,033$ & 0,017 & $-0,016$ & & $\Delta E_{\text {Inter }}^{b}$ & 0,137 & 0,155 & 0,293 \\
\hline & $\Delta E^{b}$ & 0,037 & 0,028 & 0,065 & & $\Delta E^{b}$ & 0,081 & 0,039 & 0,120 \\
\hline \multirow[t]{5}{*}{$\mathrm{A}^{1} \Pi$} & $E_{\text {Intra }}$ & $-241,646$ & $-2572,376$ & $-2814,023$ & $\mathrm{~A}^{1} \Pi$ & $E_{\text {Intra }}$ & $-37,389$ & $-397,103$ & $-434,492$ \\
\hline & $E_{\text {Inter }}$ & 0,216 & 1,476 & 1,691 & & $E_{\text {Inter }}$ & $-0,474$ & 0,324 & $-0,149$ \\
\hline & $E^{a}$ & $-241,431$ & $-2570,901$ & $-2812,331$ & & $E^{a}$ & $-37,862$ & $-396,779$ & $-434,641$ \\
\hline & $\Delta E_{\text {Intra }}{ }^{b}$ & $-0,039$ & 0,028 & $-0,011$ & & $\Delta E_{\text {Intra }}{ }^{b}$ & $-0,108$ & $-0,118$ & $-0,226$ \\
\hline & $\Delta E_{\text {Inter }}^{b}$ & 0,058 & 0,108 & 0,165 & & $\Delta E_{\text {Inter }}^{b}$ & 0,261 & 0,164 & 0,425 \\
\hline
\end{tabular}




\begin{tabular}{|c|c|c|c|c|c|c|c|c|c|}
\hline \multirow[t]{2}{*}{ Sistema/Estado } & \multirow{2}{*}{$\begin{array}{l}\text { Prop. } \\
\Delta E^{b}\end{array}$} & \multicolumn{2}{|c|}{ Átomos } & \multirow{2}{*}{$\begin{array}{r}\text { Soma } \\
0,154\end{array}$} & \multirow[t]{2}{*}{ Sistema/Estado } & \multirow{2}{*}{$\frac{\text { Prop. }}{\Delta E^{b}}$} & \multicolumn{2}{|c|}{ Átomos } & \multirow{2}{*}{$\frac{\text { Soma }}{0,199}$} \\
\hline & & 0,019 & 0,135 & & & & 0,153 & 0,046 & \\
\hline
\end{tabular}

${ }^{\mathrm{a}} E(\Omega)=E_{\text {Intra }}(\Omega)-E_{\text {Inter }}(\Omega)$.

${ }^{\mathrm{b}} \Delta E=E$ (estado excitado $)-E($ estado fundamental $)$.

A excitação eletrônica para os estados excitados tripleto das moléculas $\mathrm{AlH}, \mathrm{AlF}, \mathrm{AlCl}$ e $\mathrm{AlBr}$ acarreta em um aumento nas energias intra-atômicas totais de 0,020 a 0,089 u. a., sendo que esta desestabilização é maior para os átomos de alumínio $\left(\Delta E_{\text {Intra }}(\mathrm{Al})=0,022\right.$ a 0,082 u. a.) e quase não se observam mudanças expressivas em $E_{\text {Intra }}$ do outro átomo de cada molécula. Por sua vez, as energias interatômicas totais praticamente não variam ( $\mathrm{AlH}$ e $\mathrm{AlF}$ ) ou diminuem (estabilização) em, aproximadamente, 0,02 u. a. (AlCl e AlBr). Para os estados excitados tripletos de $\mathrm{BF}, \mathrm{BCl}, \mathrm{BBr}$ e $\mathrm{CS}$, por sua vez, observa-se o oposto, isto é, as energias intra-atômicas totais diminuem de 0,001 a 0,173 u. a. e as interatômicas totais aumentam de 0,040 a 0,293 u. a. Para estes últimos casos, os halogênios e o enxofre tendem a se estabilizar mais, apresentando $\Delta E_{\text {Intra }}$ de $-0,027$ a $-0,117$ u. a., com alterações muito menores para o boro e o carbono.

No que se referem aos estados singletos, as energias intra- e interatômicas totais aumentam de 0,021 a 0,086 u. a. e de 0,099 a 0,157, respectivamente, para AlF, $\mathrm{AlCl}, \mathrm{BF}$ e $\mathrm{BCl}$ e ambos os átomos ficam mais desestabilizados individualmente, exceto pelo boro do $\mathrm{BCl}$, que apresenta uma pequena estabilização. Já para $\mathrm{AlH}, \mathrm{AlBr}$ e CS, as energias intraatômicas totais diminuem (de 0,011 a 0,226 u. a.) e os átomos se estabilizam durante o processo de excitação eletrônica, com exceção do $\mathrm{Br}$ do AlBr. No entanto, a interação entre os átomos é fortemente desestabilizada durante esta excitação (de 0,165 a 0,425 u. a.).

De modo geral, a excitação eletrônica do estado fundamental para os estados $T_{1}$ e $S_{1}$ resulta no enfraquecimento das ligações químicas das moléculas consideradas, que é maior para estados $\mathrm{A}^{1} \Pi$ do que para a ${ }^{3} \Pi$. Isto é coerente com a redução da densidade eletrônica nos BCPs e o deslocamento batocrômico observado nas frequências vibracionais (ver seções 4.5 e 4.6).

\subsection{Comprimentos de ligação nos estados fundamentais e excitados}

Os resultados obtidos em nível de cálculo PBE0/aug-cc-pVTZ concordam satisfatoriamente com os dados experimentais, sendo que o maior desvio é referente ao estado $\mathrm{S}_{1}$ do AlCl (0,091 $\AA$ ), como mostrado na Tabela 22. Excluindo-se este caso, o segundo maior desvio em distâncias de equilíbrio é observado para o estado $S_{1}$ do $\operatorname{AlBr}(0,038 \AA)$. 
Entretanto, estas são duas das maiores distâncias de ligação encontradas, de forma que estas discrepâncias não chegam a ser graves.

Tabela 22 - Distâncias de ligação para os estados fundamentais e excitados, obtidas a partir de cálculos PBE0/aug-cc-pVTZ, e resultados experimentais disponíveis (em $\AA$ )

\begin{tabular}{|c|c|c|c|c|c|}
\hline \multirow[b]{2}{*}{ Sistema/Estado } & \multicolumn{2}{|c|}{ PBE0/aug-cc-pVTZ } & \multicolumn{2}{|c|}{ Exp } & \multirow[b]{2}{*}{ Desv. } \\
\hline & $r_{\mathrm{e}}$ & $\Delta r_{\mathrm{e}}$ & $r_{\mathrm{e}}$ & $\Delta r_{\mathrm{e}}$ & \\
\hline \multicolumn{6}{|l|}{$\mathrm{AlH}$} \\
\hline $\mathrm{X}^{1} \Sigma^{+}$ & 1,669 & & 1,648 & & 0,021 \\
\hline$a^{3} \Pi$ & 1,586 & $-0,083$ & 1,608 & $-0,040$ & $-0,022$ \\
\hline $\mathrm{A}^{1} \Pi$ & 1,673 & 0,004 & 1,648 & 0,000 & 0,025 \\
\hline \multicolumn{6}{|l|}{$\mathrm{AlF}$} \\
\hline $\mathrm{X}^{1} \Sigma^{+}$ & 1,676 & & 1,654 & & 0,021 \\
\hline$a^{3} \Pi$ & 1,668 & $-0,008$ & 1,648 & $-0,007$ & 0,020 \\
\hline $\mathrm{A}^{1} \Pi$ & 1,670 & $-0,006$ & 1,649 & $-0,006$ & 0,021 \\
\hline \multicolumn{6}{|l|}{$\mathrm{AlCl}$} \\
\hline $\mathrm{X}^{1} \Sigma^{+}$ & 2,155 & & 2,130 & & 0,025 \\
\hline$a^{3} \Pi$ & 2,111 & $-0,044$ & 2,100 & $-0,030$ & 0,011 \\
\hline $\mathrm{A}^{1} \Pi$ & 2,158 & 0,003 & 2,060 & $-0,070$ & 0,091 \\
\hline \multicolumn{6}{|l|}{$\mathrm{AlBr}$} \\
\hline $\mathrm{X}^{1} \Sigma^{+}$ & 2,324 & & 2,295 & & 0,026 \\
\hline$a^{3} \Pi$ & 2,268 & $-0,056$ & 2,260 & $-0,035$ & 0,008 \\
\hline $\mathrm{A}^{1} \Pi$ & 2,360 & 0,036 & 2,322 & 0,027 & 0,038 \\
\hline \multicolumn{6}{|l|}{$\mathrm{BF}$} \\
\hline $\mathrm{X}^{1} \Sigma^{+}$ & 1,263 & & 1,263 & & 0,000 \\
\hline$a^{3} \Pi$ & 1,319 & 0,056 & 1,308 & 0,046 & 0,011 \\
\hline $\mathrm{A}^{1} \Pi$ & 1,298 & 0,035 & 1,304 & 0,041 & $-0,006$ \\
\hline \multicolumn{6}{|l|}{$\mathrm{BCl}$} \\
\hline $\mathrm{X}^{1} \Sigma^{+}$ & 1,721 & & 1,715 & & 0,005 \\
\hline$a^{3} \Pi$ & 1,700 & $-0,021$ & 1,698 & $-0,017$ & 0,002 \\
\hline $\mathrm{A}^{1} \Pi$ & 1,680 & $-0,040$ & 1,689 & $-0,026$ & $-0,009$ \\
\hline \multicolumn{6}{|l|}{$\mathrm{BBr}$} \\
\hline $\mathrm{X}^{1} \Sigma^{+}$ & 1,895 & & 1,888 & & 0,007 \\
\hline$a^{3} \Pi$ & 1,846 & $-0,049$ & 1,853 & $-0,035$ & $-0,007$ \\
\hline $\mathrm{A}^{1} \Pi$ & 1,844 & $-0,051$ & & & \\
\hline \multicolumn{6}{|l|}{$\mathrm{CS}$} \\
\hline $\mathrm{X}^{1} \Sigma^{+}$ & 1,533 & & 1,535 & & $-0,002$ \\
\hline$a^{3} \Pi$ & 1,568 & 0,034 & 1,568 & 0,033 & $-0,001$ \\
\hline $\mathrm{A}^{1} \Pi$ & 1,573 & 0,006 & 1,573 & 0,038 & 0,000 \\
\hline
\end{tabular}

${ }^{a}$ NATIONAL INSTITUTE OF STANDARDS E TECHNOLOGY. Chemistry webbook. U. S. Secretary of Commerce: the standard reference data act. 1968. Disponível em: <http//www.nist.gov/index.html>. Acesso em: 05 dez. 2017. 
${ }^{\mathrm{b}} \Delta r_{e}=r_{e}$ (estado excitado $)-r_{e}($ estado fundamental $)$.

Em geral, os cálculos preveem corretamente o aumento ou redução do comprimento de ligação de equilíbrio durante a excitação, quando comparados aos dados experimentais. A única discrepância qualitativa ocorre no estado $\mathrm{S}_{1}$ do $\mathrm{AlCl}$, em que os cálculos indicam um leve aumento para $r_{e}(\mathrm{AlCl})$, de $0,003 \AA$, enquanto os dados experimentais apontam para uma redução significativa desta distância, de 0,070 Å. Segundo os cálculos, nos casos do AlH, $\mathrm{AlCl}$ e AlBr, a excitação leva a uma diminuição de 0,083, 0,044 e 0,056 Å, respectivamente, nas distâncias de ligação para os estados tripletos e a um aumento de 0,004, 0,003 e 0,036 desta propriedade para os estados singletos excitados. Para o $\mathrm{BCl}, \mathrm{BBr}$ e $\mathrm{AlF}$, a excitação eletrônica acarreta um encurtamento do comprimento de ligação para ambos os estados excitados, sendo que esta diminuição é maior, exceto para o AlF, para os estados singletos $(0,040$ e $0,050 \AA$ Å). Já, para o BF e CS, ocorre um aumento da distância de ligação na excitação para ambos os estados, sendo este mais pronunciado para os estados tripletos $(0,056$ e $0,034 \AA$, respectivamente) do que para os estados excitados singletos $(0,035$ e $0,006 \AA$, respectivamente).

\subsection{Momentos de dipolo e quadrupolo atômicos}

Os resultados PBE0/aug-cc-pVTZ obtidos para os momentos de dipolo de todos os estados eletrônicos das moléculas de $\mathrm{AlH}, \mathrm{AlF}, \mathrm{AlCl}, \mathrm{AlBr}, \mathrm{BF}, \mathrm{BCl}, \mathrm{BBr}$ e $\mathrm{CS}$ são encontrados na Tabela 23. Estas moléculas foram dispostas ao longo do eixo Cartesiano $z$, com o alumínio, o boro e o carbono na origem e o hidrogênio, os halogênios e o enxofre no sentido positivo deste eixo. As magnitudes experimentais dos momentos de dipolo encontrados para os estados fundamentais das moléculas AlF e CS, 1,53 e 1,96 D (HAYNES, 2016), respectivamente, concordam de forma satisfatória com os resultados teóricos. 
Tabela 23 - Momentos de dipolo $(\vec{\mu})$ e seus componentes, polarização atômica $\left(\vec{\mu}_{\mathrm{AP}}\right)$ e transferência de carga $\left(\vec{\mu}_{\mathrm{CT}}\right)$, obtidos a partir de cálculos PBE0/aug-cc-pVTZ nas geometrias de equilíbrio do estado fundamental e excitados para as moléculas estudadas (em D)

\begin{tabular}{|c|c|c|c|c|c|c|c|}
\hline Sistema/Estado & $\vec{\mu}$ & & $\Delta \vec{\mu}^{\mathrm{a}}$ & Sistema/Estado & $\vec{\mu}$ & & $\Delta \vec{\mu}^{\mathrm{a}}$ \\
\hline $\mathrm{AlH}$ & & & & $\mathrm{BF}$ & & & \\
\hline \multirow[t]{3}{*}{$\mathrm{X}^{1} \Sigma^{+}$} & $\vec{\mu}_{\mathrm{AP}}(\Omega)$ & 6,21 & & $\mathrm{X}^{1} \Sigma^{+}$ & $\vec{\mu}_{\mathrm{AP}}(\Omega)$ & 6,44 & \\
\hline & $\vec{\mu}_{\text {СТ }}(\Omega)$ & $-6,32$ & & & $\vec{\mu}_{\mathrm{CT}}(\Omega)$ & $-5,44$ & \\
\hline & $\vec{\mu}(\Omega)$ & $-0,11$ & & & $\vec{\mu}(\Omega)$ & 1,00 & \\
\hline \multirow[t]{3}{*}{$a^{3} \Pi$} & $\vec{\mu}_{\mathrm{AP}}(\Omega)$ & 4,11 & $-2,10$ & $a^{3} \Pi$ & $\vec{\mu}_{\mathrm{AP}}(\Omega)$ & 4,48 & $-1,96$ \\
\hline & $\vec{\mu}_{\text {СТ }}(\Omega)$ & $-4,89$ & 1,43 & & $\vec{\mu}_{\text {СТ }}(\Omega)$ & $-5,33$ & 0,11 \\
\hline & $\vec{\mu}(\Omega)$ & $-0,78$ & $-0,67$ & & $\mu(\Omega)$ & $-0,85$ & $-1,85$ \\
\hline \multirow[t]{3}{*}{$\mathrm{A}^{1} \Pi$} & $\vec{\mu}_{\mathrm{AP}}(\Omega)$ & 3,34 & $-2,87$ & $\mathrm{~A}^{1} \Pi$ & $\vec{\mu}_{\mathrm{AP}}(\Omega)$ & 5,52 & $-0,92$ \\
\hline & $\vec{\mu}_{\text {СТ }}(\Omega)$ & $-3,65$ & 2,68 & & $\vec{\mu}_{\text {СТ }}(\Omega)$ & $-5,32$ & 0,12 \\
\hline & $\vec{\mu}(\Omega)$ & $-0,31$ & $-0,19$ & & $\vec{\mu}(\Omega)$ & 0,20 & $-0,81$ \\
\hline $\mathrm{AlF}$ & & & & $\mathrm{BCl}$ & & & \\
\hline \multirow[t]{3}{*}{$\mathrm{X}^{1} \Sigma^{+}$} & $\vec{\mu}_{\mathrm{AP}}(\Omega)$ & 6,01 & & $\mathrm{X}^{1} \Sigma^{+}$ & $\vec{\mu}_{\mathrm{AP}}(\Omega)$ & 7,85 & \\
\hline & $\vec{\mu}_{\text {СТ }}(\Omega)$ & $-7,52$ & & & $\vec{\mu}_{\text {СТ }}(\Omega)$ & $-6,29$ & \\
\hline & $\vec{\mu}(\Omega)$ & $-1,51$ & & & $\vec{\mu}(\Omega)$ & 1,56 & \\
\hline \multirow[t]{3}{*}{$a^{3} \Pi$} & $\vec{\mu}_{\mathrm{AP}}(\Omega)$ & 5,25 & $-0,76$ & $a^{3} \Pi$ & $\vec{\mu}_{\mathrm{AP}}(\Omega)$ & 4,91 & $-2,94$ \\
\hline & $\vec{\mu}_{\text {СТ }}(\Omega)$ & $-7,18$ & 0,33 & & $\vec{\mu}_{\text {СТ }}(\Omega)$ & $-5,77$ & 0,53 \\
\hline & $\vec{\mu}(\Omega)$ & $-1,94$ & $-0,43$ & & $\mu(\Omega)$ & $-0,85$ & $-2,42$ \\
\hline \multirow[t]{3}{*}{$\mathrm{A}^{1} \Pi$} & $\vec{\mu}_{\mathrm{AP}}(\Omega)$ & 5,77 & $-0,24$ & $\mathrm{~A}^{1} \Pi$ & $\vec{\mu}_{\mathrm{AP}}(\Omega)$ & 5,97 & $-1,89$ \\
\hline & $\vec{\mu}_{\text {СТ }}(\Omega)$ & $-7,01$ & 0,51 & & $\vec{\mu}_{\text {СТ }}(\Omega)$ & $-5,40$ & 0,90 \\
\hline & $\vec{\mu}(\Omega)$ & $-1,24$ & 0,27 & & $\vec{\mu}(\Omega)$ & 0,57 & $-0,99$ \\
\hline $\mathrm{AlCl}$ & & & & $\mathrm{BBr}$ & & & \\
\hline \multirow[t]{3}{*}{$\mathrm{X}^{1} \Sigma^{+}$} & $\vec{\mu}_{\mathrm{AP}}(\Omega)$ & 7,27 & & $\mathrm{X}^{1} \Sigma^{+}$ & $\vec{\mu}_{\mathrm{AP}}(\Omega)$ & 7,47 & \\
\hline & $\vec{\mu}_{\text {СТ }}(\Omega)$ & $-8,92$ & & & $\vec{\mu}_{\text {СТ }}(\Omega)$ & $-6,00$ & \\
\hline & $\vec{\mu}(\Omega)$ & $-1,65$ & & & $\vec{\mu}(\Omega)$ & 1,47 & \\
\hline \multirow[t]{3}{*}{$a^{3} \Pi$} & $\vec{\mu}_{\mathrm{AP}}(\Omega)$ & 6,53 & $-0,74$ & $a^{3} \Pi$ & $\vec{\mu}_{\mathrm{AP}}(\Omega)$ & 4,49 & $-2,98$ \\
\hline & $\vec{\mu}_{\text {СТ }}(\Omega)$ & $-8,34$ & 0,58 & & $\vec{\mu}_{\text {СТ }}(\Omega)$ & $-5,23$ & 0,77 \\
\hline & $\vec{\mu}(\Omega)$ & $-1,81$ & $-0,16$ & & $\vec{\mu}(\Omega)$ & $-0,74$ & $-2,21$ \\
\hline \multirow[t]{3}{*}{$\mathrm{A}^{1} \Pi$} & $\vec{\mu}_{\mathrm{AP}}(\Omega)$ & 6,40 & $-0,86$ & $\mathrm{~A}^{1} \Pi$ & $\vec{\mu}_{\mathrm{AP}}(\Omega)$ & 4,95 & $-2,51$ \\
\hline & $\vec{\mu}_{\text {СТ }}(\Omega)$ & $-7,19$ & 1,73 & & $\vec{\mu}_{\text {СТ }}(\Omega)$ & $-4,08$ & 1,92 \\
\hline & $\vec{\mu}(\Omega)$ & $-0,79$ & 0,86 & & $\vec{\mu}(\Omega)$ & 0,87 & $-0,60$ \\
\hline $\mathrm{AlBr}$ & & & & CS & & & \\
\hline \multirow[t]{3}{*}{$\mathrm{X}^{1} \Sigma^{+}$} & $\vec{\mu}_{\mathrm{AP}}(\Omega)$ & 7,50 & & $\mathrm{X}^{1} \Sigma^{+}$ & $\vec{\mu}_{\mathrm{AP}}(\Omega)$ & $-3,44$ & \\
\hline & $\vec{\mu}_{\mathrm{CT}}(\Omega)$ & $-9,25$ & & & $\vec{\mu}_{\mathrm{CT}}(\Omega)$ & 5,35 & \\
\hline & $\vec{\mu}(\Omega)$ & $-1,75$ & & & $\vec{\mu}(\Omega)$ & 1,90 & \\
\hline \multirow[t]{2}{*}{$a^{3} \Pi$} & $\vec{\mu}_{\mathrm{AP}}(\Omega)$ & 6,77 & $-0,73$ & $a^{3} \Pi$ & $\vec{\mu}_{\mathrm{AP}}(\Omega)$ & $-4,34$ & $-0,89$ \\
\hline & $\vec{\mu}_{\mathrm{CT}}(\Omega)$ & $-8,43$ & 0,82 & & $\vec{\mu}_{\mathrm{CT}}(\Omega)$ & 4,03 & $-1,32$ \\
\hline
\end{tabular}




\begin{tabular}{cccccccc}
\hline Sistema/Estado & $\vec{\mu}$ & & $\Delta \vec{\mu}^{\mathrm{a}}$ & Sistema/Estado & $\vec{\mu}$ & $\Delta \vec{\mu}^{\mathrm{a}}$ \\
\hline \multirow{4}{*}{$\mathrm{A}^{1} \Pi$} & $\vec{\mu}(\Omega)$ & $-1,66$ & 0,09 & & $\vec{\mu}(\Omega)$ & $-0,31$ & $-2,21$ \\
& $\vec{\mu}_{\mathrm{AP}}(\Omega)$ & 5,73 & $-1,77$ & $\mathrm{~A}^{1} \Pi$ & $\vec{\mu}_{\mathrm{AP}}(\Omega)$ & $-3,53$ & $-0,09$ \\
& $\vec{\mu}_{\mathrm{CT}}(\Omega)$ & $-6,27$ & 2,98 & & $\vec{\mu}_{\mathrm{CT}}(\Omega)$ & 3,99 & $-1,36$ \\
& $\vec{\mu}(\Omega)$ & $-0,54$ & 1,21 & & $\vec{\mu}(\Omega)$ & 0,46 & $-1,45$ \\
\hline
\end{tabular}

${ }^{\text {a }} \Delta \vec{\mu}=\vec{\mu}$ (estado excitado $)-\vec{\mu}$ (estado fundamental).

A excitação eletrônica acarreta um aumento da magnitude do momento de dipolo para os estados tripletos e uma diminuição no valor desta propriedade para os estados singletos excitados das moléculas $\mathrm{AlF}$ e $\mathrm{AlCl}$, sem inversão de sinais. No caso do $\mathrm{AlBr}$, observam-se reduções da magnitude do vetor de momento de dipolo, sem mudanças de sinais, tanto para $\mathrm{S}_{1}$ quanto para $\mathrm{T}_{1}$. Para o $\mathrm{AlH}$, a intensificação do vetor de momento de dipolo ocorre para ambos os estados excitados. Já, para os demais sistemas, $\mathrm{BF}, \mathrm{BCl}, \mathrm{BBr}$ e CS, observa-se reduções de $|\vec{\mu}|$ para ambos os estados excitados e, além disso, ocorre uma inversão no sentido do vetor para os estados tripletos. Como esperado pelo mecanismo de polarização contra polarização (BADER, 1990), as contribuições de transferência de carga (CT) e de polarização atômica (AP) têm sinais opostos em todos os estados. Além disto, a contribuição CT geralmente é predominante, determinando o sinal do momento de dipolo. As exceções ocorrem para os estados fundamentais e $\mathrm{S}_{1}$ do $\mathrm{BF}, \mathrm{BCl}$, e do $\mathrm{BBr}$ e para o estado $\mathrm{T}_{1}$ do $\mathrm{CS}$, nos quais os dipolos atômicos determinam o sinal do momento de dipolo.

As contribuições de transferência de carga e de polarização atômica tendem a diminuir em magnitude para quase todos os estados excitados quando se compara com os respectivos estados fundamentais (a exceção fica por conta da polarização atômica nos estados excitados do CS). A redução em $\vec{\mu}_{\mathrm{z}, \mathrm{CT}}$ é mais expressiva para os estados excitados singletos do que para os estados tripletos (isto também é observado para a molécula de CS), enquanto observa-se o oposto para $\vec{\mu}_{z, \mathrm{AP}}$, exceto para os sistemas $\mathrm{AlH}, \mathrm{AlCl}$ e $\mathrm{AlBr}$ (em que ambas as contribuições diminuem mais em excitações para $S_{1}$ ). Entretanto, $\vec{\mu}_{\mathrm{z}, \mathrm{CT}}$ varia muito pouco nos três estados dos sistemas BF e AlF, sendo que as mudanças de magnitude e sentido nestes casos são muito dependentes do que ocorre com a contribuição de polarização atômica.

A Tabela 24 lista de forma mais detalhada os momentos de dipolo calculados com PBE0/aug-cc-pVTZ e suas decomposições em polarização atômica $\left(\vec{\mu}_{z, \mathrm{AP}}(\Omega)\right)$ e transferência de carga $\left(\vec{\mu}_{\mathrm{z}, \text { Ст }}(\Omega)\right)$, assim como suas variações durante a excitação eletrônica. A excitação eletrônica provoca uma redução da separação de cargas atômicas nos estados excitados de todos os sistemas considerados em relação ao estado fundamental $\left(\mathrm{AlH}: 0,79 e\right.$ para $\mathrm{X}^{1} \Sigma^{+}$, 
0,64 e para a ${ }^{3} \Pi$ e $0,45 e$ para $\mathrm{A}^{1} \Pi$, por exemplo), as quais, entretanto, ainda mantêm os mesmos sinais que para o estado fundamental (alumínio, boro e enxofre positivamente carregados e hidrogênio, halogênios e carbono negativamente carregados). Isto explica por que a contribuição de transferência de carga eletrônica diminui de magnitude em todos os casos, mas ainda apresenta o mesmo sinal para os três estados de cada molécula.

Tabela 24 - Decomposição atômica do momento de dipolo $(\vec{\mu})$ dos sistemas $\mathrm{AlH}, \mathrm{AlF}, \mathrm{AlCl}$, $\mathrm{AlBr}, \mathrm{BF}, \mathrm{BCl}, \mathrm{BBr}$ e CS calculados com PBE0/aug-cc-pVTZ nas geometrias de equilíbrio de cada estado (As cargas estão em u. a. e os momentos de dipolo em D)

\begin{tabular}{|c|c|c|c|c|c|c|c|c|c|}
\hline \multirow[t]{2}{*}{ Estado } & \multirow[t]{2}{*}{ Propriedade } & \multicolumn{2}{|c|}{ Átomos $(\Omega)$} & \multirow[t]{2}{*}{ Soma } & \multirow[t]{2}{*}{ Estado } & \multirow[t]{2}{*}{ Propriedade } & \multicolumn{2}{|c|}{ Átomos $(\Omega)$} & \multirow[t]{2}{*}{ Soma } \\
\hline & & $\mathrm{Al}$ & $\mathrm{H}$ & & & & B & $\mathrm{F}$ & \\
\hline \multirow[t]{4}{*}{$\mathrm{X}^{1} \Sigma^{+}$} & $\mathrm{q}(\Omega)$ & 0,789 & $-0,789$ & 0,000 & $\mathrm{X}^{1} \Sigma^{+}$ & $\mathrm{q}(\Omega)$ & 0,897 & $-0,897$ & 0,000 \\
\hline & $\mu_{\mathrm{z}, \mathrm{AP}}(\Omega)$ & 5,306 & 0,907 & 6,213 & & $\mu_{\mathrm{z}, \mathrm{AP}}(\Omega)$ & 4,522 & 1,918 & 6,440 \\
\hline & $\mu_{\mathrm{z}, \mathrm{CT}}(\Omega)$ & 0,000 & $-6,325$ & $-6,325$ & & $\mu_{\mathrm{z}, \mathrm{CT}}(\Omega)$ & 0,008 & $-5,447$ & $-5,439$ \\
\hline & $\mu_{\mathrm{z}}(\Omega)$ & & & $-0,112$ & & $\mu_{\mathrm{z}}(\Omega)$ & & & 1,001 \\
\hline \multirow[t]{8}{*}{$a^{3} \Pi$} & $\mathrm{q}(\Omega)$ & 0,643 & $-0,642$ & 0,000 & $a^{3} \Pi$ & $\mathrm{q}(\Omega)$ & 0,842 & $-0,842$ & 0,000 \\
\hline & $\mu_{\mathrm{z}, \mathrm{AP}}(\Omega)$ & 3,134 & 0,978 & 4,111 & & $\mu_{\mathrm{z}, \mathrm{AP}}(\Omega)$ & 2,990 & 1,489 & 4,479 \\
\hline & $\mu_{\mathrm{z}, \mathrm{CT}}(\Omega)$ & 0,000 & $-4,891$ & $-4,891$ & & $\mu_{\mathrm{z}, \mathrm{CT}}(\Omega)$ & $-0,036$ & $-5,293$ & $-5,329$ \\
\hline & $\mu_{\mathrm{z}}(\Omega)$ & & & $-0,779$ & & $\vec{\mu}_{z}(\Omega)$ & & & $-0,850$ \\
\hline & $\Delta \mathrm{q}(\Omega)^{\mathrm{a}}$ & $-0,147$ & 0,147 & 0,000 & & $\Delta \mathrm{q}(\Omega)^{\mathrm{a}}$ & $-0,055$ & 0,055 & 0,000 \\
\hline & $\Delta \mu_{\mathrm{z}, \mathrm{AP}}(\Omega)^{\mathrm{a}}$ & $-2,173$ & 0,071 & $-2,102$ & & $\Delta \mu_{z, \mathrm{AP}}(\Omega)^{\mathrm{a}}$ & $-1,532$ & $-0,429$ & $-1,961$ \\
\hline & $\Delta \mu_{\mathrm{z}, \mathrm{CT}}(\Omega)^{\mathrm{a}}$ & 0,000 & 1,434 & 1,434 & & $\Delta \mu_{\mathrm{z}, \mathrm{CT}}(\Omega)^{\mathrm{a}}$ & $-0,044$ & 0,154 & 0,110 \\
\hline & $\Delta \mu_{\mathrm{z}}(\Omega)^{\mathrm{a}}$ & & & $-0,668$ & & $\Delta \mu_{\mathrm{z}}(\Omega)^{\mathrm{a}}$ & & & $-1,851$ \\
\hline \multirow[t]{9}{*}{$\mathrm{A}^{1} \Pi$} & $\mathrm{q}(\Omega)$ & 0,454 & $-0,454$ & 0,000 & $\mathrm{~A}^{1} \Pi$ & $\mathrm{q}(\Omega)$ & 0,855 & $-0,854$ & 0,000 \\
\hline & $\mu_{\mathrm{z}, \mathrm{AP}}(\Omega)$ & 2,618 & 0,722 & 3,340 & & $\mu_{\mathrm{z}, \mathrm{AP}}(\Omega)$ & 3,613 & 1,904 & 5,518 \\
\hline & $\mu_{\mathrm{z}, \mathrm{CT}}(\Omega)$ & 0,000 & $-3,646$ & $-3,646$ & & $\mu_{\mathrm{z}, \mathrm{CT}}(\Omega)$ & 0,012 & $-5,335$ & $-5,322$ \\
\hline & $\mu_{\mathrm{z}}(\Omega)$ & & & $-0,306$ & & $\mu_{\mathrm{z}}(\Omega)$ & & & 0,195 \\
\hline & $\Delta \mathrm{q}(\Omega)^{\mathrm{a}}$ & $-0,335$ & 0,335 & 0,000 & & $\Delta \mathrm{q}(\Omega)^{\mathrm{a}}$ & $-0,042$ & 0,043 & 0,000 \\
\hline & $\Delta \mu_{\mathrm{z}, \mathrm{AP}}(\Omega)^{\mathrm{a}}$ & $-2,688$ & $-0,185$ & $-2,873$ & & $\Delta \mu_{z, \mathrm{AP}}(\Omega)^{\mathrm{a}}$ & $-0,908$ & $-0,014$ & $-0,922$ \\
\hline & $\Delta \mu_{\mathrm{z}, \mathrm{CT}}(\Omega)^{\mathrm{a}}$ & 0,000 & 2,679 & 2,679 & & $\Delta \mu_{\mathrm{z}, \mathrm{CT}}(\Omega)^{\mathrm{a}}$ & 0,004 & 0,113 & 0,116 \\
\hline & $\Delta \mu_{\mathrm{z}}(\Omega)^{\mathrm{a}}$ & & & $-0,195$ & & $\Delta \mu_{\mathrm{z}}(\Omega)^{\mathrm{a}}$ & & & $-0,806$ \\
\hline & & $\mathrm{Al}$ & $\mathrm{F}$ & & & & B & $\mathrm{Cl}$ & \\
\hline \multirow[t]{4}{*}{$\mathrm{X}^{1} \Sigma^{+}$} & $\mathrm{q}(\Omega)$ & 0,935 & $-0,934$ & 0,000 & $\mathrm{X}^{1} \Sigma^{+}$ & $\mathrm{q}(\Omega)$ & 0,762 & $-0,762$ & 0,000 \\
\hline & $\mu_{\mathrm{z}, \mathrm{AP}}(\Omega)$ & 4,777 & 1,234 & 6,011 & & $\mu_{\mathrm{z}, \mathrm{AP}}(\Omega)$ & 4,440 & 3,413 & 7,854 \\
\hline & $\mu_{\mathrm{z}, \mathrm{CT}}(\Omega)$ & $-0,048$ & $-7,470$ & $-7,518$ & & $\mu_{\mathrm{z}, \mathrm{CT}}(\Omega)$ & $-0,010$ & $-6,282$ & $-6,292$ \\
\hline & $\mu_{\mathrm{z}}(\Omega)$ & & & $-1,507$ & & $\mu_{z}(\Omega)$ & & & 1,562 \\
\hline \multirow[t]{2}{*}{$a^{3} \Pi$} & $\mathrm{q}(\Omega)$ & 0,897 & $-0,897$ & 0,000 & $a^{3} \Pi$ & $\mathrm{q}(\Omega)$ & 0,706 & $-0,706$ & 0,000 \\
\hline & $\mu_{\mathrm{z}, \mathrm{AP}}(\Omega)$ & 3,988 & 1,259 & 5,247 & & $\mu_{\mathrm{z}, \mathrm{AP}}(\Omega)$ & 2,457 & 2,454 & 4,911 \\
\hline
\end{tabular}




\begin{tabular}{|c|c|c|c|c|c|c|c|c|c|}
\hline \multirow[t]{2}{*}{ Estado } & \multirow{2}{*}{$\frac{\text { Propriedade }}{\mu_{\mathrm{z}, \mathrm{CT}}(\Omega)}$} & \multicolumn{2}{|c|}{ Átomos $(\Omega)$} & \multirow{2}{*}{$\begin{array}{c}\text { Soma } \\
-7,184\end{array}$} & \multirow[t]{2}{*}{ Estado } & \multirow{2}{*}{$\begin{array}{c}\text { Propriedade } \\
\mu_{\mathrm{z}, \mathrm{CT}}(\Omega)\end{array}$} & \multicolumn{2}{|c|}{ Átomos $(\Omega)$} & \multirow{2}{*}{$\begin{array}{r}\text { Soma } \\
-5,765\end{array}$} \\
\hline & & $-0,043$ & $-7,141$ & & & & $-0,003$ & $-5,762$ & \\
\hline & $\mu_{\mathrm{z}}(\Omega)$ & & & $-1,937$ & & $\mu_{\mathrm{z}}(\Omega)$ & & & $-0,854$ \\
\hline & $\Delta \mathrm{q}(\Omega)^{\mathrm{a}}$ & $-0,038$ & 0,037 & $-0,001$ & & $\Delta \mathrm{q}(\Omega)^{\mathrm{a}}$ & $-0,055$ & 0,055 & 0,000 \\
\hline & $\Delta \mu_{\mathrm{z}, \mathrm{AP}}(\Omega)^{\mathrm{a}}$ & $-0,789$ & 0,024 & $-0,764$ & & $\Delta \mu_{\mathrm{z}, \mathrm{AP}}(\Omega)^{\mathrm{a}}$ & $-1,983$ & $-0,960$ & $-2,943$ \\
\hline & $\Delta \mu_{\mathrm{z}, \mathrm{CT}}(\Omega)^{\mathrm{a}}$ & 0,005 & 0,330 & 0,334 & & $\Delta \mu_{\mathrm{z}, \mathrm{CT}}(\Omega)^{\mathrm{a}}$ & 0,007 & 0,520 & 0,527 \\
\hline & $\Delta \mu_{\mathrm{z}}(\Omega)^{\mathrm{a}}$ & & & $-0,430$ & & $\Delta \mu_{\mathrm{z}}(\Omega)^{\mathrm{a}}$ & & & $-2,416$ \\
\hline \multirow[t]{9}{*}{$\mathrm{A}^{1} \Pi$} & $\mathrm{q}(\Omega)$ & 0,878 & $-0,874$ & 0,004 & $\mathrm{~A}^{1} \Pi$ & $\mathrm{q}(\Omega)$ & 0,669 & $-0,669$ & 0,000 \\
\hline & $\mu_{\mathrm{z}, \mathrm{AP}}(\Omega)$ & 4,323 & 1,446 & 5,769 & & $\mu_{\mathrm{z}, \mathrm{AP}}(\Omega)$ & 3,149 & 2,816 & 5,966 \\
\hline & $\mu_{\mathrm{z}, \mathrm{CT}}(\Omega)$ & $-0,045$ & $-6,961$ & $-7,006$ & & $\mu_{\mathrm{z}, \mathrm{CT}}(\Omega)$ & 0,014 & $-5,410$ & $-5,396$ \\
\hline & $\mu_{\mathrm{z}}(\Omega)$ & & & $-1,237$ & & $\mu_{\mathrm{z}}(\Omega)$ & & & 0,570 \\
\hline & $\Delta \mathrm{q}(\Omega)^{\mathrm{a}}$ & $-0,057$ & 0,061 & 0,004 & & $\Delta \mathrm{q}(\Omega)^{\mathrm{a}}$ & $-0,093$ & 0,093 & 0,000 \\
\hline & $\Delta \mu_{\mathrm{z}, \mathrm{AP}}(\Omega)^{\mathrm{a}}$ & $-0,454$ & 0,212 & $-0,242$ & & $\Delta \mu_{z, \mathrm{AP}}(\Omega)^{\mathrm{a}}$ & $-1,291$ & $-0,597$ & $-1,888$ \\
\hline & $\Delta \mu_{z, \mathrm{CT}}(\Omega)^{\mathrm{a}}$ & 0,003 & 0,509 & 0,512 & & $\Delta \mu_{\mathrm{z}, \mathrm{CT}}(\Omega)^{\mathrm{a}}$ & 0,024 & 0,872 & 0,896 \\
\hline & $\Delta \mu_{\mathrm{z}}(\Omega)^{\mathrm{a}}$ & & & 0,270 & & $\Delta \mu_{\mathrm{z}}(\Omega)^{\mathrm{a}}$ & & & $-0,992$ \\
\hline & & $\mathrm{Al}$ & $\mathrm{Cl}$ & & & & B & $\mathrm{Br}$ & \\
\hline \multirow[t]{4}{*}{$\mathrm{X}^{1} \Sigma^{+}$} & $\mathrm{q}(\Omega)$ & 0,862 & $-0,862$ & 0,000 & $\mathrm{X}^{1} \Sigma^{+}+$ & $\mathrm{q}(\Omega)$ & 0,659 & $-0,659$ & 0,000 \\
\hline & $\mu_{\mathrm{z}, \mathrm{AP}}(\Omega)$ & 4,596 & 2,672 & 7,268 & & $\mu_{\mathrm{z}, \mathrm{AP}}(\Omega)$ & 4,147 & 3,319 & 7,466 \\
\hline & $\mu_{\mathrm{z}, \mathrm{CT}}(\Omega)$ & $-0,052$ & $-8,866$ & $-8,917$ & & $\mu_{\mathrm{z}, \mathrm{CT}}(\Omega)$ & $-0,011$ & $-5,985$ & $-5,996$ \\
\hline & $\mu_{\mathrm{z}}(\Omega)$ & & & $-1,649$ & & $\mu_{\mathrm{z}}(\Omega)$ & & & 1,470 \\
\hline \multirow[t]{8}{*}{$a^{3} \Pi$} & $\mathrm{q}(\Omega)$ & 23 & $-0,823$ & 0,000 & $\Pi$ & $\mathrm{q}(\Omega$ &, 590 & $-0,590$ & 0,000 \\
\hline & $\mu_{\mathrm{z}, \mathrm{AP}}(\Omega)$ & 3,728 & 2,800 & 6,528 & & $\mu_{\mathrm{z}, \mathrm{AP}}(\Omega)$ & 2,137 & 2,349 & 4,486 \\
\hline & $\mu_{\mathrm{z}, \mathrm{CT}}(\Omega)$ & $-0,021$ & $-8,316$ & $-8,337$ & & $\mu_{\mathrm{z}, \mathrm{CT}}(\Omega)$ & 0,005 & $-5,232$ & $-5,226$ \\
\hline & $\mu_{\mathrm{z}}(\Omega)$ & & & $-1,809$ & & $\mu_{\mathrm{z}}(\Omega)$ & & & $-0,740$ \\
\hline & $\Delta \mathrm{q}(\Omega)^{\mathrm{a}}$ & $-0,039$ & 0,039 & 0,000 & & $\Delta \mathrm{q}(\Omega)^{\mathrm{a}}$ & $-0,069$ & 0,070 & 0,000 \\
\hline & $\Delta \mu_{z, \mathrm{AP}}(\Omega)^{\mathrm{a}}$ & $-0,868$ & 0,128 & $-0,740$ & & $\Delta \mu_{z, \mathrm{AP}}(\Omega)^{\mathrm{a}}$ & $-2,010$ & $-0,970$ & $-2,980$ \\
\hline & $\Delta \mu_{\mathrm{z}, \mathrm{CT}}(\Omega)^{\mathrm{a}}$ & 0,030 & 0,550 & 0,580 & & $\Delta \mu_{\mathrm{z}, \mathrm{CT}}(\Omega)^{\mathrm{a}}$ & 0,016 & 0,754 & 0,770 \\
\hline & $\Delta \mu_{\mathrm{z}}(\Omega)^{\mathrm{a}}$ & & & $-0,160$ & & $\Delta \mu_{\mathrm{z}}(\Omega)^{\mathrm{a}}$ & & & $-2,210$ \\
\hline \multirow[t]{9}{*}{$\mathrm{A}^{1} \Pi$} & $\mathrm{q}(\Omega)$ & 0,695 & $-0,694$ & 0,001 & $\mathrm{~A}^{1} \Pi$ & $\mathrm{q}(\Omega)$ & 0,461 & $-0,461$ & 0,000 \\
\hline & $\mu_{\mathrm{z}, \mathrm{AP}}(\Omega)$ & 3 & 2,751 & 6,404 & & $\mu_{z, \mathrm{AP}(\Omega)}$ & 2,681 & 2,271 & 4,952 \\
\hline & $\mu_{\mathrm{z}, \mathrm{CT}}(\Omega)$ & $-0,163$ & $-7,027$ & $-7,190$ & & $\mu_{\mathrm{z}, \mathrm{CT}}(\Omega)$ & 0,028 & $-4,106$ & $-4,078$ \\
\hline & $\mu_{z}(\Omega)$ & & & $-0,786$ & & $\mu_{\mathrm{z}}(\Omega)$ & & & 0,874 \\
\hline & $\Delta \mathrm{q}(\Omega)^{\mathrm{a}}$ & $-0,167$ & 0,168 & 0,001 & & $\Delta \mathrm{q}(\Omega)^{\mathrm{a}}$ & $-0,198$ & 0,199 & 0,000 \\
\hline & $\Delta \mu_{\mathrm{z}, \mathrm{AP}}(\Omega)^{\mathrm{a}}$ & $-0,943$ & 0,078 & $-0,864$ & & $\Delta \mu_{z, \mathrm{AP}}(\Omega)^{\mathrm{a}}$ & $-1,466$ & $-1,048$ & $-2,514$ \\
\hline & $\Delta \mu_{\mathrm{z}, \mathrm{CT}}(\Omega)^{\mathrm{a}}$ & $-0,112$ & 1,839 & 1,727 & & $\Delta \mu_{\mathrm{z}, \mathrm{CT}}(\Omega)^{\mathrm{a}}$ & 0,039 & 1,879 & 1,918 \\
\hline & $\Delta \mu_{\mathrm{z}}(\Omega)^{\mathrm{a}}$ & & & 0,863 & & $\Delta \mu_{\mathrm{z}}(\Omega)^{\mathrm{a}}$ & & & $-0,595$ \\
\hline & & 10 & (1) & & & & e & 3 & \\
\hline \multirow[t]{2}{*}{$\mathrm{X}^{1} \Sigma^{+}$} & $\mathrm{q}(\Omega)$ & 0,829 & $-0,829$ & 0,000 & $\mathrm{X}^{1} \Sigma^{+}$ & $\mathrm{q}(\Omega)$ & $-0,726$ & 0,726 & 0,000 \\
\hline & $\mu_{\mathrm{z}, \mathrm{AP}}(\Omega)$ & 4,437 & 3,064 & 7,500 & & $\mu_{\mathrm{z}, \mathrm{AP}}(\Omega)$ & $-0,468$ & $-2,976$ & $-3,444$ \\
\hline
\end{tabular}




\begin{tabular}{|c|c|c|c|c|c|c|c|c|c|}
\hline \multirow[t]{2}{*}{ Estado } & \multirow{2}{*}{$\begin{array}{c}\text { Propriedade } \\
\mu_{\mathrm{z}, \mathrm{CT}}(\Omega)\end{array}$} & \multicolumn{2}{|c|}{ Átomos $(\Omega)$} & \multirow{2}{*}{$\begin{array}{c}\text { Soma } \\
-9,249\end{array}$} & \multirow[t]{2}{*}{ Estado } & \multirow{2}{*}{$\begin{array}{c}\text { Propriedade } \\
\mu_{\mathrm{z}, \mathrm{CT}}(\Omega)\end{array}$} & \multicolumn{2}{|c|}{ Átomos $(\Omega)$} & \multirow{2}{*}{$\begin{array}{l}\text { Soma } \\
5,348\end{array}$} \\
\hline & & $-0,058$ & $-9,191$ & & & & $-0,003$ & 5,351 & \\
\hline & $\mu_{\mathrm{z}}(\Omega)$ & & & $-1,749$ & & $\mu_{\mathrm{z}}(\Omega)$ & & & 1,904 \\
\hline \multirow[t]{8}{*}{$a^{3} \Pi$} & $\mathrm{q}(\Omega)$ & 0,774 & $-0,774$ & 0,000 & $a^{3} \Pi$ & $\mathrm{q}(\Omega)$ & $-0,535$ & 0,535 & 0,000 \\
\hline & $\mu_{\mathrm{z}, \mathrm{AP}}(\Omega)$ & 3,565 & 3,206 & 6,772 & & $\mu_{z, \mathrm{AP}}(\Omega)$ & $-1,411$ & $-2,926$ & $-4,337$ \\
\hline & $\mu_{\mathrm{z}, \mathrm{CT}}(\Omega)$ & $-0,126$ & $-8,304$ & $-8,430$ & & $\mu_{\mathrm{z}, \mathrm{CT}}(\Omega)$ & $-0,001$ & 4,029 & 4,028 \\
\hline & $\mu_{\mathrm{z}}(\Omega)$ & & & $-1,659$ & & $\mu_{\mathrm{z}}(\Omega)$ & & & $-0,309$ \\
\hline & $\Delta \mathrm{q}(\Omega)^{\mathrm{a}}$ & $-0,055$ & 0,055 & 0,000 & & $\Delta \mathrm{q}(\Omega)^{\mathrm{a}}$ & 0,191 & $-0,191$ & 0,000 \\
\hline & $\Delta \mu_{\mathrm{z}, \mathrm{AP}}(\Omega)^{\mathrm{a}}$ & $-0,872$ & 0,143 & $-0,729$ & & $\Delta \mu_{z, \mathrm{AP}}(\Omega)^{\mathrm{a}}$ & $-0,943$ & 0,050 & $-0,893$ \\
\hline & $\Delta \mu_{\mathrm{z}, \mathrm{CT}}(\Omega)^{\mathrm{a}}$ & $-0,068$ & 0,887 & 0,819 & & $\Delta \mu_{\mathrm{z}, \mathrm{CT}}(\Omega)^{\mathrm{a}}$ & 0,003 & $-1,322$ & $-1,320$ \\
\hline & $\Delta \mu_{\mathrm{z}}(\Omega)^{\mathrm{a}}$ & & & 0,090 & & $\Delta \mu_{\mathrm{z}}(\Omega)^{\mathrm{a}}$ & & & $-2,213$ \\
\hline \multirow[t]{8}{*}{$\mathrm{A}^{1} \Pi$} & $\mathrm{q}(\Omega)$ & 0,554 & $-0,553$ & 0,001 & A ${ }^{1} \Pi$ & $\mathrm{q}(\Omega)$ & $-0,528$ & 0,528 & 0,000 \\
\hline & $\mu_{\mathrm{z}, \mathrm{AP}}(\Omega)$ & 3,099 & 2,633 & 5,732 & & $\mu_{z, \mathrm{AP}}(\Omega)$ & $-0,860$ & $-2,673$ & $-3,533$ \\
\hline & $\mu_{z, \mathrm{CT}}(\Omega)$ & $-0,050$ & $-6,219$ & $-6,269$ & & $\mu_{\mathrm{z}, \mathrm{CT}}(\Omega)$ & 0,000 & 3,990 & 3,990 \\
\hline & $\mu_{\mathrm{z}}(\Omega)$ & & & $-0,537$ & & $\mu_{\mathrm{z}}(\Omega)$ & & & 0,457 \\
\hline & $\Delta \mathrm{q}(\Omega)^{\mathrm{a}}$ & $-0,275$ & 0,276 & 0,001 & & $\Delta \mathrm{q}(\Omega)^{\mathrm{a}}$ & 0,198 & $-0,198$ & 0,000 \\
\hline & $\Delta \mu_{\mathrm{z}, \mathrm{AP}}(\Omega)^{\mathrm{a}}$ & $-1,338$ & $-0,431$ & $-1,769$ & & $\Delta \mu_{\mathrm{z}, \mathrm{AP}}(\Omega)^{\mathrm{a}}$ & $-0,392$ & 0,303 & $-0,089$ \\
\hline & $\Delta \mu_{\mathrm{z}, \mathrm{CT}}(\Omega)^{\mathrm{a}}$ & 0,008 & 2,972 & 2,980 & & $\Delta \mu_{\mathrm{z}, \mathrm{CT}}(\Omega)^{\mathrm{a}}$ & 0,003 & $-1,361$ & $-1,358$ \\
\hline & $\Delta \mu_{\mathrm{z}}(\Omega)^{\mathrm{a}}$ & & & 1,212 & & $\Delta \mu_{\mathrm{z}}(\Omega)^{\mathrm{a}}$ & & & $-1,446$ \\
\hline
\end{tabular}

As variações que ocorrem na contribuição da transferência de carga dos haletos de boro são menos expressivas que aquelas de polarização atômica. No caso da molécula de BF, as mudanças em $\vec{\mu}_{\mathrm{z}, \mathrm{CT}}$ de cada átomo são quase nulas. Por sua vez, analisando as contribuições $\vec{\mu}_{z, \mathrm{AP}}$, notamos que o momento de dipolo do boro é a principal quantidade afetada pela excitação, diminuindo de 1,53 e $0,91 \mathrm{D}$ durante a excitação para $\mathrm{T}_{1}$ e $\mathrm{S}_{1}$, respectivamente (veja que o momento de dipolo total desta molécula cai em 1,85 e $0,81 \mathrm{D}$ na transição de $\mathrm{S}_{0}$ para $T_{1}$ e $S_{1}$, respectivamente). Portanto, é a grande redução no dipolo atômico do boro que explica a significativa diminuição de magnitude do momento de dipolo do BF no processo $\mathrm{S}_{0}$ $\rightarrow S_{1}$ e também a inversão no sentido deste vetor no processo $S_{0} \rightarrow T_{1}$. A redução do dipolo atômico do boro também é o principal fator a ser considerado para $\mathrm{BCl}$ e $\mathrm{BBr}$, com valores entre $-1,98$ e -2,01 D no caso da excitação $S_{0} \rightarrow T_{1}$ e entre $-1,29$ e $-1,47$ D para $S_{0} \rightarrow S_{1}$. A diferença é que os dipolos atômicos do cloro e do bromo sofrem uma redução mais significativa que aquela do flúor, enquanto a contribuição CT tem sentido oposto, cancelando em grande parte (estados $T_{1}$ ) ou superando um pouco (estados $S_{1}$ ) a contribuição AP destes halogênios mais polarizáveis. Novamente, a grande redução de dipolo atômico do boro na 
transição para os estados tripletos explica a inversão do sentido do dipolo atômico observada no $\mathrm{BCl}$ e $\mathrm{BBr}$, enquanto a diminuição menos expressiva do dipolo atômico do boro na excitação para os estados $S_{1}$ não é capaz de inverter o sentido deste vetor, mas resulta em uma grande redução de sua magnitude.

Quanto à molécula de CS, os átomos de carbono apresentam valores de polarização atômica bem mais negativos nos estados excitados $\left(\mathrm{T}_{1}: \vec{\mu}_{\mathrm{z}, \mathrm{AP}}(\mathrm{C})=-1,41 \mathrm{D} ; \mathrm{S}_{1}: \vec{\mu}_{\mathrm{z}, \mathrm{AP}}(\mathrm{C})=-0,86\right.$ D) do que no estado fundamental $\left(\mathrm{S}_{0}: \vec{\mu}_{z, \mathrm{AP}}(\mathrm{C})=-0,47 \mathrm{D}\right)$, enquanto os valores desta quantidade para os átomos de enxofre sofrem um aumento muito pequeno durante a excitação eletrônica. Então, as mudanças de polarização do carbono predominam sobre aquelas do enxofre, principalmente no caso do estado tripleto. Por sua vez, a redução da contribuição CT é quase a mesma nos processos $S_{0} \rightarrow T_{1}$ e $S_{0} \rightarrow S_{1}$ (ao redor de -1,32 e -1,36 D). Desta forma, a inversão de sentido do momento de dipolo do CS no estado $\mathrm{T}_{1}$ ocorre por conta da maior redução do dipolo atômico do carbono nesta excitação, enquanto a diminuição deste dipolo atômico no estado $S_{1}$ não é suficiente para inverter o sentido do dipolo da molécula de CS.

Para a molécula de $\mathrm{AlH}$, a variação das contribuições AP é similar nas excitações para $\mathrm{S}_{1}$ ou $\mathrm{T}_{1},-2,87$ e $-2,10 \mathrm{D}$, respectivamente, com predominância da contribuição AP do alumínio (-2,69 e -2,17 D, respectivamente). Por sua vez, a contribuição CT aumenta o dipolo molecular, sendo muito maior sua contribuição para o estado $\mathrm{S}_{1}(2,68 \mathrm{D})$ do que para o $\mathrm{T}_{1}$ (1,43 D). Como ambos os efeitos, CT e AP, quase se cancelam para o estado $\mathrm{S}_{1}$, o momento de dipolo do $\mathrm{AlH}$ neste caso é semelhante aquele determinado para o estado fundamental. Entretanto, as duas contribuições não se cancelam de forma tão eficiente para o estado $\mathrm{T}_{1}$, intensificando o momento de dipolo negativo neste caso. Ou seja, as separações de carga distintas observadas nos estados $\mathrm{S}_{1}$ e $\mathrm{T}_{1}$ são responsáveis pelas mudanças observadas nos momentos de dipolo desta molécula durante ambas as excitações.

Por sua vez, as variações das contribuições CT nas excitações do AlF são pequenas e positivas $\left(0,33\right.$ e $0,51 \mathrm{D}$ para $\mathrm{T}_{1}$ e $\mathrm{S}_{1}$, respectivamente). Além disto, a variação do momento de dipolo do alumínio durante as excitações (-0,79 e -0,45 D para $T_{1}$ e $S_{1}$, respectivamente) é muito mais relevante que aquela do flúor. Ambos estes efeitos tendem a um cancelamento parcial no caso de estado $S_{1}$, enquanto há uma intensificação do momento de dipolo negativo do AlF na transição para $\mathrm{T}_{1}$. Em outras palavras, isto ocorre, principalmente, por conta das mudanças de polarização do alumínio durante as excitações para ambos os estados estudados.

Para as moléculas de $\mathrm{AlCl}$ e $\mathrm{AlBr}$, o termo de transferência de carga eletrônica tem papel muito distinto dependendo se nos referimos ao estado $S_{1}$ ou $T_{1}$. Então, a variação da 
contribuição CT é muito menor no processo $\mathrm{S}_{0} \rightarrow \mathrm{T}_{1}(0,58$ e $0,82 \mathrm{D}$ respectivamente no $\mathrm{AlCl}$ e no $\mathrm{AlBr}$ ) que no processo $\mathrm{S}_{0} \rightarrow \mathrm{S}_{1}(1,73$ e 2,98 $\mathrm{D}$ respectivamente no $\mathrm{AlCl}$ e no $\mathrm{AlBr}$ ). Enquanto a variação das contribuições AP é quase a mesma nos dois estados excitados do $\mathrm{AlCl}$, com predominância daquela do dipolo atômico do alumínio $(-0,87$ e $-0,94 \mathrm{D}$ respectivamente para $T_{1}$ e $S_{1}$ ), a variação do dipolo atômico do alumínio do AlBr é também predominante e assume valores de $-0,87$ e $-1,34 \mathrm{D}$, respectivamente, nos processos $\mathrm{S}_{0} \rightarrow \mathrm{T}_{1}$ e $\mathrm{S}_{0} \rightarrow \mathrm{S}_{1}$. Desta maneira, a intensificação do dipolo negativo do $\mathrm{AlCl}$ durante a excitação para $\mathrm{T}_{1}$ e o leve enfraquecimento desta quantidade na transição para $\mathrm{S}_{1}$ ocorrem, principalmente, por conta das diferentes separações de carga em ambos estes estados. No caso do AlBr, as mudanças são mais complicadas e se devem, principalmente, às diferenças nas separações de carga, embora as polarizações distintas do alumínio em ambos os estados excitados também tenham papel importante neste caso.

Na Tabela 25 constam os elementos $Q_{z z}$ dos momentos de quadrupolo atômicos dos sistemas estudados. Esta propriedade é uma medida da deformação da nuvem eletrônica de cada átomo a partir de uma simetria esférica (eixo $z$ em relação ao plano $x y$ ). Os momentos de quadrupolo dos átomos aumentam durante a excitação eletrônica, exceto pelo $Q_{z z}$ do flúor, que diminui um pouco nos estados excitados tripletos do BF e AlF. Os momentos de quadrupolo variam de forma mais pronunciada para os átomos de alumínio, boro e carbono do que para os átomos de hidrogênio, halogênios e enxofre e, nestes casos, o efeito da excitação tende a ser maior para o estado singleto do que para o tripleto. Isto indica que todas as excitações devem ser do tipo $\sigma \rightarrow \pi$, pois um valor mais positivo de $Q_{z z}$ implica que a nuvem eletrônica do átomo tem agora uma distribuição mais concentrada ao longo do plano xy em relação ao eixo molecular $(z)$ do que ocorria para o estado fundamental. Além disto, muitas das propriedades analisadas aqui indicam um enfraquecimento das ligações durante tais excitações (embora haja alguns poucos valores discordantes). Isto está em acordo com a classificação destas excitações como sendo do tipo $\sigma \rightarrow \pi^{*}$. Isto também está coerente com a classificação experimental dos estados excitados considerados (NATIONAL INSTITUTE OF STANDARDS E TECHNOLOGY, 1968).

Tabela 25 - Momentos de quadrupolo atômico QTAIM ao longo do eixo molecular de ligação $\left(Q_{z z}\right)$ obtidos em cálculos PBE0/aug-cc-pVTZ das moléculas $\mathrm{AlH}, \mathrm{AlF}, \mathrm{AlCl}, \mathrm{AlBr}$, $\mathrm{BF}, \mathrm{BCl}, \mathrm{BBr}$ e $\mathrm{CS}$ (em u. a.)

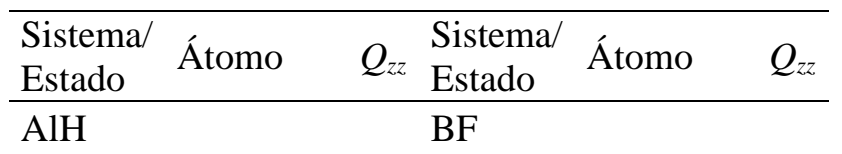




\begin{tabular}{|c|c|c|c|c|c|}
\hline $\begin{array}{l}\text { Sistema/ } \\
\text { Estado } \\
\end{array}$ & Átomo & $Q_{z z}$ & $\begin{array}{l}\text { Sistema/ } \\
\text { Estado }\end{array}$ & Átomo & $Q_{z z}$ \\
\hline \multirow[t]{2}{*}{$\mathrm{X}^{1} \Sigma^{+}$} & $\mathrm{Al}$ & $-0,770$ & $\mathrm{X}^{1} \Sigma^{+}$ & B & $-0,051$ \\
\hline & $\mathrm{H}$ & 0,848 & & $\mathrm{~F}$ & 0,137 \\
\hline \multirow[t]{2}{*}{$a^{3} \Pi$} & $\mathrm{Al}$ & 6,944 & $a^{3} \Pi$ & B & 2,596 \\
\hline & $\mathrm{H}$ & 1,420 & & $\mathrm{~F}$ & 0,061 \\
\hline \multirow[t]{2}{*}{$\mathrm{A}^{1} \Pi$} & $\mathrm{Al}$ & 7,510 & $\mathrm{~A}^{1} \Pi$ & B & 4,564 \\
\hline & $\mathrm{H}$ & 1,136 & & $\mathrm{~F}$ & 0,262 \\
\hline $\mathrm{AlF}$ & & & $\mathrm{BCl}$ & & \\
\hline \multirow[t]{2}{*}{$X^{1} \Sigma^{+}$} & $\mathrm{Al}$ & 1,050 & $\mathrm{X}^{1} \Sigma^{+}$ & B & $-0,079$ \\
\hline & $\mathrm{F}$ & 0,808 & & $\mathrm{Cl}$ & $-1,633$ \\
\hline \multirow[t]{2}{*}{$a^{3} \Pi$} & $\mathrm{Al}$ & 6,106 & $a^{3} \Pi$ & B & 2,605 \\
\hline & $\mathrm{F}$ & 0,660 & & $\mathrm{Cl}$ & $-0,071$ \\
\hline \multirow[t]{2}{*}{$\mathrm{A}^{1} \Pi$} & $\mathrm{Al}$ & 9,486 & $\mathrm{~A}^{1} \Pi$ & B & 2,483 \\
\hline & $\mathrm{F}$ & 0,965 & & $\mathrm{Cl}$ & $-0,293$ \\
\hline $\mathrm{AlCl}$ & & & $\mathrm{BBr}$ & & \\
\hline \multirow[t]{2}{*}{$\mathrm{X}^{1} \Sigma^{+}$} & $\mathrm{Al}$ & 0,880 & $\mathrm{X}^{1} \Sigma^{+}$ & B & 0,129 \\
\hline & $\mathrm{Cl}$ & $-0,410$ & & $\mathrm{Br}$ & $-1,623$ \\
\hline \multirow[t]{2}{*}{$a^{3} \Pi$} & $\mathrm{Al}$ & 5,914 & $a^{3} \Pi$ & B & 2,853 \\
\hline & $\mathrm{Cl}$ & $-0,232$ & & $\mathrm{Br}$ & 0,662 \\
\hline \multirow[t]{2}{*}{$\mathrm{A}^{1} \Pi$} & $\mathrm{Al}$ & 7,819 & $\mathrm{~A}^{1} \Pi$ & B & 2,875 \\
\hline & $\mathrm{Cl}$ & 0,272 & & $\mathrm{Br}$ & 0,729 \\
\hline $\mathrm{AlBr}$ & & & CS & & \\
\hline \multirow[t]{2}{*}{$\mathrm{X}^{1} \Sigma^{+}$} & $\mathrm{Al}$ & 0,879 & $\mathrm{X}^{1} \Sigma^{+}$ & $\mathrm{C}$ & $-0,791$ \\
\hline & $\mathrm{Br}$ & $-0,891$ & & $\mathrm{~S}$ & 5,056 \\
\hline \multirow[t]{2}{*}{$a^{3} \Pi$} & $\mathrm{Al}$ & 5,942 & $a^{3} \Pi$ & $\mathrm{C}$ & 1,264 \\
\hline & $\mathrm{Br}$ & $-0,481$ & & $S$ & 6,145 \\
\hline \multirow[t]{2}{*}{$\mathrm{A}^{1} \Pi$} & $\mathrm{Al}$ & 7,712 & $\mathrm{~A}^{1} \Pi$ & $\mathrm{C}$ & 1,609 \\
\hline & $\mathrm{Br}$ & 0,633 & & $S$ & 5,833 \\
\hline
\end{tabular}

\subsection{Propriedades topológicas QTAIM}

A Tabela 26 apresenta os resultados de algumas propriedades topológicas nos pontos críticos das ligações Al-H, Al-F, Al-Cl, Al-Br, B-F, B-Cl, B-Br e C-S como, por exemplo, densidade eletrônica $\left(\rho_{B C P}\right)$, Laplaciano da densidade eletrônica $\left(\nabla^{2} \rho_{B C P}\right)$ e densidade de energia eletrônica $\left(H_{b}\right)$, obtidas com o nível de cálculo PBE0/aug-cc-pVTZ. As excitações verticais ocasionam reduções nas densidades eletrônicas nos BCPs das ligações estudadas, exceto para os estados tripletos das moléculas $\mathrm{AlF}, \mathrm{AlCl}$ e $\mathrm{AlBr}$, nas quais esta propriedade permanece constante. No caso do BF, as variações das densidades eletrônicas são as mesmas 
para os estados excitados tripleto e singleto $(0,004$ u. a.). Entretanto, de modo geral, a redução da densidade eletrônica é mais pronunciada para estados singletos.

Tabela 26 - Propriedades topológicas QTAIM nos pontos críticos de ligação calculadas em nível PBE0/aug-cc-pVTZ para processos de excitação verticais e adiabáticos (em u. a.) ${ }^{\mathrm{a}}$

\begin{tabular}{|c|c|c|c|c|}
\hline Sistema/Estado & $r(\AA)$ & $\rho_{B C P}$ & $\nabla^{2} \rho_{B C P}$ & $H_{b}=\left(G_{b}+V_{b}\right)$ \\
\hline \multicolumn{5}{|l|}{$\mathrm{AlH}$} \\
\hline $\mathrm{X}^{1} \Sigma^{+}$ & 1,669 & 0,073 & 0,168 & $-0,024$ \\
\hline \multicolumn{5}{|l|}{ Vertical } \\
\hline$a^{3} \Pi$ & 1,669 & 0,068 & 0,164 & $-0,020$ \\
\hline $\mathrm{A}^{1} \Pi$ & 1,669 & 0,063 & 0,155 & $-0,018$ \\
\hline \multicolumn{5}{|l|}{ Adiabático } \\
\hline$a^{3} \Pi$ & 1,586 & 0,078 & 0,237 & $-0,023$ \\
\hline $\mathrm{A}^{1} \Pi$ & 1,673 & 0,063 & 0,152 & $-0,017$ \\
\hline \multicolumn{5}{|l|}{$\mathrm{AlF}$} \\
\hline $\mathrm{X}^{1} \Sigma^{+}$ & 1,676 & 0,103 & 0,961 & $-0,001$ \\
\hline \multicolumn{5}{|l|}{ Vertical } \\
\hline $\mathrm{a}^{3} \Pi$ & 1,676 & 0,104 & 0,954 & $-0,002$ \\
\hline $\mathrm{A}^{1} \Pi$ & 1,676 & 0,103 & 0,935 & $-0,003$ \\
\hline \multicolumn{5}{|l|}{ Adiabático } \\
\hline$a^{3} \Pi$ & 1,668 & 0,106 & 0,983 & $-0,002$ \\
\hline $\mathrm{A}^{1} \Pi$ & 1,670 & 0,105 & 0,956 & $-0,003$ \\
\hline \multicolumn{5}{|l|}{$\mathrm{AlCl}$} \\
\hline $\mathrm{X}^{1} \Sigma^{+}$ & 2,155 & 0,066 & 0,269 & $-0,014$ \\
\hline \multicolumn{5}{|l|}{ Vertical } \\
\hline$a^{3} \Pi$ & 2,155 & 0,066 & 0,270 & $-0,014$ \\
\hline $\mathrm{A}^{1} \Pi$ & 2,155 & 0,064 & 0,248 & $-0,014$ \\
\hline \multicolumn{5}{|l|}{ Adiabático } \\
\hline$a^{3} \Pi$ & 2,111 & 0,072 & 0,315 & $-0,015$ \\
\hline $\mathrm{A}^{1} \Pi$ & 2,158 & 0,064 & 0,245 & $-0,014$ \\
\hline \multicolumn{5}{|l|}{$\mathrm{AlBr}$} \\
\hline $\mathrm{X}^{1} \Sigma^{+}$ & 2,324 & 0,058 & 0,162 & $-0,016$ \\
\hline \multicolumn{5}{|l|}{ Vertical } \\
\hline$a^{3} \Pi$ & 2,324 & 0,058 & 0,165 & $-0,016$ \\
\hline $\mathrm{A}^{1} \Pi$ & 2,324 & 0,055 & 0,143 & $-0,015$ \\
\hline \multicolumn{5}{|l|}{ Adiabático } \\
\hline $\mathrm{a}^{3} \Pi$ & 2,268 & 0,064 & 0,204 & $-0,017$ \\
\hline $\mathrm{A}^{1} \Pi$ & 2,360 & 0,052 & 0,121 & $-0,014$ \\
\hline \multicolumn{5}{|l|}{$\mathrm{BF}$} \\
\hline $\mathrm{X}^{1} \Sigma^{+}$ & 1,263 & 0,242 & 1,544 & $-0,193$ \\
\hline
\end{tabular}




\begin{tabular}{lrrrr}
\hline Sistema/Estado & $r(\AA)$ & $\rho_{B C P}$ & $\nabla^{2} \rho_{B C P}$ & $H_{b}=\left(G_{b}+V_{b}\right)$ \\
\hline Vertical & & & & \\
a $^{3} \Pi$ & 1,263 & 0,238 & 1,801 & $-0,182$ \\
A ${ }^{1} \Pi$ & 1,263 & 0,238 & 1,773 & $-0,185$
\end{tabular}

Adiabático

\begin{tabular}{lllll}
$\mathrm{a}^{3} \Pi$ & 1,319 & 0,206 & 1,406 & $-0,152$ \\
$\mathrm{~A}{ }^{1} \Pi$ & 1,298 & 0,216 & 1,525 & $-0,163$ \\
\hline $\mathrm{BCl}$ & & & & \\
$\mathrm{X}^{1} \Sigma^{+}$ & 1,720 & 0,156 & $-0,008$ & $-0,152$
\end{tabular}

Vertical

$\begin{array}{lllll}\mathrm{a}^{3} \Pi & 1,720 & 0,152 & 0,200 & -0,144 \\ \mathrm{~A}^{1} \Pi & 1,720 & 0,147 & 0,234 & -0,135\end{array}$

Adiabático

\begin{tabular}{lllll}
$\mathrm{a}^{3} \Pi$ & 1,700 & 0,158 & 0,231 & $-0,151$ \\
$\mathrm{~A}^{1} \Pi$ & 1,680 & 0,193 & 0,296 & $-0,148$ \\
\hline $\mathrm{BBr}$ & & & & \\
$\mathrm{X}^{1} \Sigma^{+}$ & 1,895 & 0,137 & $-0,212$ & $-0,133$
\end{tabular}

Vertical

$\begin{array}{lllll}\text { a }^{3} \Pi & 1,895 & 0,133 & -0,040 & -0,126\end{array}$

$\begin{array}{lllll}\mathrm{A}^{1} \Pi & 1,895 & 0,125 & -0,008 & -0,115\end{array}$

Adiabático

\begin{tabular}{lllll}
$\mathrm{a}^{3} \Pi$ & 1,846 & 0,143 & 0,006 & $-0,139$ \\
$\mathrm{~A}^{1} \Pi$ & 1,844 & 0,136 & 0,042 & $-0,129$ \\
\hline $\mathrm{CS}$ & & & & \\
$\mathrm{X}^{1} \Sigma^{+}$ & 1,533 & 0,286 & 0,207 & $-0,390$
\end{tabular}

Vertical

$\begin{array}{lllll}\mathrm{a}^{3} \Pi & 1,533 & 0,251 & 0,285 & -0,320 \\ \mathrm{~A}^{1} \Pi & 1,533 & 0,243 & 0,199 & -0,312\end{array}$

Adiabático

$\begin{array}{lllll}\text { a }^{3} \Pi \quad 1,568 & 0,237 & 0,118 & -0,298\end{array}$

$\begin{array}{lllll}\mathrm{A}^{1} \Pi \quad 1,573 & 0,228 & -0,001 & -0,286\end{array}$

${ }^{a}$ Os símbolos usados para as propriedades de ligação avaliadas para o BCP são: $\rho_{B C P}$ para a densidade eletrônica, $\nabla^{2} \rho_{B C P}$ é o Laplaciano da densidade eletrônica, e $G_{B C P}, V_{B C P}$ e $H_{\mathrm{BCP}}$ são, respectivamente, a densidade de energia cinética, a densidade de energia potencial e a densidade de energia total.

Em relação às excitações verticais dos sistemas contendo alumínio, observa-se que o valor do $\nabla^{2} \rho_{B C P}$ é menor para os estados excitados do que para o estado fundamental, isto é, a concentração da densidade eletrônica no BCP da ligação aumenta com a excitação eletrônica, exceto para os estados tripletos do $\mathrm{AlCl}$ e $\mathrm{AlBr}$, em que há um leve aumento desta propriedade. Já, para as espécies $\mathrm{BF}, \mathrm{BCl}$ e $\mathrm{BBr}$, o valor do Laplaciano da densidade 
eletrônica aumenta durante a excitação vertical para os dois estados excitados considerados, indicando que a concentração de densidade eletrônica nos BCPs tende a diminuir por conta das transições. $\mathrm{O}$ aumento mais significativo ocorre para o estado tripleto no primeiro caso e para o singleto nos dois últimos casos. O CS, por sua vez, apresenta um maior valor de $\nabla^{2} \rho_{B C P}$ para o estado tripleto, enquanto quase não há variação durante a excitação para o singleto. As densidades de energia totais são negativas, indicando o predomínio da energia potencial sobre a cinética e seus valores absolutos diminuem ou praticamente não se alteram durante as transições verticais.

\subsection{Frequências vibracionais}

As frequências vibracionais harmônicas para os estados fundamentais e excitados das moléculas estudadas são apresentadas na Tabela 27. Os maiores desvios são de 295 e $63 \mathrm{~cm}^{-1}$, para os estados $\mathrm{A}^{1} \Pi$ do $\mathrm{AlH}$ e $\mathrm{BBr}$, respectivamente. Em geral, os cálculos predizem corretamente a direção e a magnitude dos deslocamentos observados na posição das bandas vibracionais, em comparação aos dados experimentais. Entretanto, os cálculos subestimam significativamente o deslocamento no caso do estado $\mathrm{S}_{1}$ do $\mathrm{AlH}$ (teo.: $-261 \mathrm{~cm}^{-1}$ e exp.: -600 $\mathrm{cm}^{-1}$ ). Para os estados excitados singletos das moléculas $\mathrm{AlH}, \mathrm{AlF}, \mathrm{AlCl}$ e $\mathrm{AlBr}$, as frequências vibracionais são mais baixas do que aquelas nos estados fundamentais, indicando um deslocamento batocrômico, enquanto os valores para esta propriedade são maiores para os estados tripletos destes casos, evidenciando um deslocamento hipsocrômico. Além disso, o deslocamento batocrômico é observado para ambos os estados das moléculas BF e CS, enquanto $\mathrm{BCl}$ e $\mathrm{BBr}$ apresentam somente deslocamentos hipsocrômicos.

Tabela 27 - Frequências vibracionais harmônicas $(\omega)$, obtidas em cálculos PBE0/aug-ccpVTZ, e resultados experimentais $\left(\mathrm{em} \mathrm{cm}^{-1}\right)$

\begin{tabular}{lccccc}
\hline & \multicolumn{2}{c}{ PBE0/aug-cc-pVTZ } & \multicolumn{2}{c}{ Exp. $^{\text {a }}$} & \\
\cline { 2 - 5 } Sistema/Estado & $\omega$ & $\Delta \omega^{\mathrm{b}}$ & $\omega$ & $\Delta \omega^{\mathrm{b}}$ & Desv. \\
\hline $\mathrm{AlH}$ & 1639 & & 1683 & & -44 \\
$\mathrm{X}^{1} \Sigma^{+}$ & 1843 & 204 & & & \\
$\mathrm{a}^{3} \Pi$ & 1378 & -261 & 1083 & -600 & 295 \\
$\mathrm{~A}^{1} \Pi$ & 773 & & 802 & & -29 \\
\hline $\mathrm{AlF}$ & 808 & 34 & 828 & 26 & -20 \\
$\mathrm{X}^{1} \Sigma^{+}$ & & & & &
\end{tabular}




\begin{tabular}{|c|c|c|c|c|c|}
\hline \multirow[b]{2}{*}{ Sistema/Estado } & \multicolumn{2}{|c|}{ PBE0/aug-cc-pVTZ } & \multicolumn{2}{|c|}{ Exp. $^{a}$} & \multirow[b]{2}{*}{ Desv. ${ }^{\mathrm{c}}$} \\
\hline & $\omega$ & $\Delta \omega^{\mathrm{b}}$ & $\omega$ & $\Delta \omega^{\mathrm{b}}$ & \\
\hline $\mathrm{A}^{1} \Pi$ & 763 & -10 & 804 & 2 & -41 \\
\hline \multicolumn{6}{|l|}{$\mathrm{AlCl}$} \\
\hline $\mathrm{X}^{1} \Sigma^{+}$ & 468 & & 481 & & -13 \\
\hline$a^{3} \Pi$ & 521 & 52 & 524 & 43 & -4 \\
\hline $\mathrm{A}^{1} \Pi$ & 407 & -61 & 450 & -31 & -43 \\
\hline \multicolumn{6}{|l|}{$\mathrm{AlBr}$} \\
\hline $\mathrm{X}^{1} \Sigma^{+}$ & 368 & & 378 & & -10 \\
\hline$a^{3} \Pi$ & 410 & 42 & 411 & 33 & -1 \\
\hline $\mathrm{A}^{1} \Pi$ & 265 & -104 & 297 & -81 & -33 \\
\hline \multicolumn{6}{|l|}{$\mathrm{BF}$} \\
\hline $\mathrm{X}^{1} \Sigma^{+}$ & 1413 & & 1402 & & 11 \\
\hline$a^{3} \Pi$ & 1317 & -96 & 1324 & -78 & -6 \\
\hline $\mathrm{A}^{1} \Pi$ & 1294 & -119 & 1265 & -137 & 29 \\
\hline \multicolumn{6}{|l|}{$\mathrm{BCl}$} \\
\hline $\mathrm{X}^{1} \Sigma^{+}$ & 836 & & 839 & & -3 \\
\hline$a^{3} \Pi$ & 941 & 106 & 911 & 72 & 30 \\
\hline $\mathrm{A}^{1} \Pi$ & 885 & -56 & 849 & 10 & 36 \\
\hline \multicolumn{6}{|l|}{$\mathrm{BBr}$} \\
\hline $\mathrm{X}^{1} \Sigma^{+}$ & 683 & & 684 & & -1 \\
\hline $\mathrm{a}^{3} \Pi$ & 798 & 115 & 757 & 73 & 41 \\
\hline $\mathrm{A}^{1} \Pi$ & 697 & 14 & 760 & 75 & -63 \\
\hline \multicolumn{6}{|l|}{ CS } \\
\hline $\mathrm{X}^{1} \Sigma^{+}$ & 1327 & & 1285 & & 42 \\
\hline$a^{3} \Pi$ & 1175 & -152 & 1135 & -150 & 40 \\
\hline $\mathrm{A}^{1} \Pi$ & 1081 & -247 & 1073 & -212 & 7 \\
\hline
\end{tabular}

${ }^{a}$ NATIONAL INSTITUTE OF STANDARDS E TECHNOLOGY. Chemistry webbook. U. S. Secretary of Commerce: the standard reference data act. 1968. Disponível em: <http//www.nist.gov/index.html>. Acesso em: 5 dez. 2017.

${ }^{b} \Delta \omega=\omega$ (estado excitado) $-\omega$ (estado fundamental).

${ }^{\mathrm{c}}$ Desv. $=\omega($ teórico $)-\omega($ exp.).

\subsection{Análise de Carga - Fluxo de Carga - Fluxo de Dipolo das intensidades de infravermelho}

As intensidades de infravermelho foram obtidas a partir das derivadas do momento de dipolo em relação aos modos normais de vibração. A partição CCFDF/QTAIM desta propriedade para as moléculas $\mathrm{AlH}, \mathrm{AlF}, \mathrm{AlCl}, \mathrm{AlBr}, \mathrm{BF}, \mathrm{BCl}, \mathrm{BBr}$ e $\mathrm{CS}$ pode ser visualizada 
na Tabela 28. As intensidades de infravermelho obtidas segundo o modelo CCFDF/QTAIM concordam satisfatoriamente com aquelas calculadas diretamente em nível PBE0/aug-ccpVTZ. As intensidades de infravermelho tendem a diminuir com a excitação eletrônica, de 33,8 a $698,3 \mathrm{~km} \mathrm{~mol}^{-1}$, exceto no caso do estado tripleto do $\mathrm{BF}$, em que há um leve aumento de $33,3 \mathrm{~km} \mathrm{~mol}^{-1}$.

Tabela 28 - Contribuições para as intensidades fundamentais de infravermelho segundo análise CCFDF/QTAIM, assim como as obtidas em cálculos PBE0/aug-cc-pVTZ, e suas variações durante a excitação eletrônica $\left(\mathrm{em} \mathrm{km} \mathrm{mol}^{-1}\right)^{\mathrm{a}}$

\begin{tabular}{|c|c|c|c|c|c|c|c|c|}
\hline \multirow{2}{*}{ Sistema/Estado } & \multicolumn{7}{|c|}{ CCFDF/QTAIM } & \multirow{2}{*}{ Int. (PBE0/aug-cc-pVTZ) } \\
\hline & $A^{C}$ & $A^{C F}$ & $A^{D F}$ & $A^{C \times C F}$ & $A^{C \times D F}$ & $A^{C F \times D F}$ & $A^{\text {Total }}$ & \\
\hline \multicolumn{9}{|l|}{$\mathrm{AlH}$} \\
\hline $\mathrm{X}^{1} \Sigma^{+}$ & 625,3 & 22,5 & 38,5 & $-237,0$ & 310,4 & $-58,8$ & 700,9 & 701,0 \\
\hline$a^{3} \Pi$ & 414,1 & 362,8 & 1,1 & $-775,2$ & $-14,5$ & 13,6 & 1,9 & 2,6 \\
\hline $\mathrm{A}^{1} \Pi$ & 206,9 & 1637,0 & 473,2 & $-1163,9$ & 622,7 & $-1751,6$ & 24,3 & 24,9 \\
\hline \multicolumn{9}{|l|}{$\mathrm{AlF}$} \\
\hline $\mathrm{X}^{1} \Sigma^{+}$ & 76,4 & 1,3 & 9,7 & $-20,0$ & 54,5 & $-7,1$ & 114,7 & 115,1 \\
\hline$a^{3} \Pi$ & 70,4 & 3,2 & 8,3 & $-29,8$ & 48,3 & $-10,2$ & 90,1 & 92,7 \\
\hline $\mathrm{A}^{1} \Pi$ & 67,1 & 35,2 & 22,1 & $-97,2$ & 77,0 & $-55,8$ & 48,4 & 48,0 \\
\hline \multicolumn{9}{|l|}{$\mathrm{AlCl}$} \\
\hline $\mathrm{X}^{1} \Sigma^{+}$ & 47,5 & 0,4 & 26,3 & $-9,2$ & 70,7 & $-6,8$ & 128,9 & 128,9 \\
\hline$a^{3} \Pi$ & 43,3 & 11,2 & 20,2 & $-44,1$ & 59,1 & $-30,1$ & 59,6 & 60,0 \\
\hline $\mathrm{A}^{1} \Pi$ & 30,9 & 179,4 & 132,1 & $-148,9$ & 127,7 & $-307,8$ & 13,4 & 14,0 \\
\hline \multicolumn{9}{|l|}{$\mathrm{AlBr}$} \\
\hline $\mathrm{X}^{1} \Sigma^{+}$ & 33,3 & 0,8 & 25,2 & $-10,1$ & 57,9 & $-8,8$ & 98,4 & 98,4 \\
\hline$a^{3} \Pi$ & 29,1 & 16,4 & 21,6 & $-43,7$ & 50,1 & $-37,7$ & 35,8 & 35,7 \\
\hline $\mathrm{A}^{1} \Pi$ & 14,9 & 214,6 & 160,7 & $-113,0$ & 97,8 & $-371,4$ & 3,6 & 3,3 \\
\hline \multicolumn{9}{|l|}{$\mathrm{BF}$} \\
\hline $\mathrm{X}^{1} \Sigma^{+}$ & 112,5 & 3,7 & 15,4 & $-40,9$ & 83,2 & $-15,1$ & 158,9 & 158,8 \\
\hline$a^{3} \Pi$ & 99,1 & 17,7 & 65,2 & $-83,9$ & 160,8 & $-68,1$ & 191,0 & 192,1 \\
\hline $\mathrm{A}^{1} \Pi$ & 102,2 & 89,8 & 111,7 & $-191,5$ & 213,6 & $-200,2$ & 125,5 & 125,0 \\
\hline \multicolumn{9}{|l|}{$\mathrm{BCl}$} \\
\hline $\mathrm{X}^{1} \Sigma^{+}$ & 67,5 & 12,1 & 117,4 & $-57,2$ & 178,1 & $-75,4$ & 242,5 & 242,5 \\
\hline$a^{3} \Pi$ & 58,1 & 72,9 & 61,3 & $-130,1$ & 119,3 & $-133,7$ & 47,8 & 47,8 \\
\hline $\mathrm{A}^{1} \Pi$ & 52,1 & 429,4 & 269,4 & $-299,2$ & 237,0 & $-680,3$ & 8,5 & 8,3 \\
\hline \multicolumn{9}{|l|}{$\mathrm{BBr}$} \\
\hline $\mathrm{X}^{1} \Sigma^{+}$ & 43,8 & 107,1 & 329,9 & $-137,0$ & 240,5 & $-375,9$ & 208,4 & 208,4 \\
\hline$a^{3} \Pi$ & 35,1 & 94,0 & 60,8 & $-114,9$ & 92,3 & $-151,2$ & 16,2 & 16,2 \\
\hline $\mathrm{A}^{1} \Pi$ & 21,4 & 554,7 & 432,7 & $-218,0$ & 192,5 & $-979,8$ & 3,5 & 3,6 \\
\hline
\end{tabular}




\begin{tabular}{|c|c|c|c|c|c|c|c|c|}
\hline \multirow{2}{*}{ Sistema/Estado } & \multicolumn{7}{|c|}{ CCFDF/QTAIM } & \multirow{2}{*}{ Int. (PBE0/aug-cc-pVTZ) } \\
\hline & $A^{C}$ & $A^{C F}$ & $A^{D F}$ & $A^{C \times C F}$ & $A^{C \times D F}$ & $A^{C F \times D F}$ & $A^{\text {Total }}$ & \\
\hline \multicolumn{9}{|l|}{$\mathrm{CS}$} \\
\hline $\mathrm{X}^{1} \Sigma^{+}$ & 59,0 & 2833,1 & 1298,9 & $-817,5$ & 553,5 & $-3836,6$ & 90,4 & 90,5 \\
\hline$a^{3} \Pi$ & 32,0 & 1973,0 & 1457,6 & $-502,6$ & 432,0 & $-3391,7$ & 0,3 & 0,3 \\
\hline $\mathrm{A}^{1} \Pi$ & 31,2 & 2468,4 & 2113,8 & $-554,9$ & 513,4 & $-4568,5$ & 3,5 & 3,5 \\
\hline Sistema/Estado & $\Delta A^{C}$ & $\Delta A^{C F}$ & $\Delta A^{D F}$ & $\Delta A^{C \times C F}$ & $\Delta A^{C \times D F}$ & $\Delta A^{C F \times D F}$ & $\Delta A^{\text {Total }}$ & $\Delta$ Int. (PBE0/aug-cc-pVTZ) \\
\hline \multicolumn{9}{|l|}{$\mathrm{AlH}$} \\
\hline$a^{3} \Pi$ & $-211,3$ & 340,3 & $-37,4$ & $-538,2$ & $-324,9$ & 72,4 & $-699,0$ & $-698,3$ \\
\hline $\mathrm{A}^{1} \Pi$ & $-418,5$ & 1614,5 & 434,7 & $-926,8$ & 312,2 & $-1692,8$ & $-676,6$ & $-676,0$ \\
\hline \multicolumn{9}{|l|}{ AlF } \\
\hline$a^{3} \Pi$ & $-6,0$ & 1,8 & $-1,4$ & $-9,8$ & $-6,2$ & $-3,1$ & $-24,6$ & $-22,4$ \\
\hline $\mathrm{A}^{1} \Pi$ & $-9,3$ & 33,9 & 12,4 & $-77,1$ & 22,5 & $-48,6$ & $-66,3$ & $-67,1$ \\
\hline \multicolumn{9}{|l|}{$\mathrm{AlCl}$} \\
\hline$a^{3} \Pi$ & $-4,2$ & 10,8 & $-6,1$ & $-34,9$ & $-11,5$ & $-23,3$ & $-69,2$ & $-68,8$ \\
\hline $\mathrm{A}^{1} \Pi$ & $-16,7$ & 178,9 & 105,8 & $-139,7$ & 57,1 & $-301,0$ & $-115,5$ & $-114,9$ \\
\hline \multicolumn{9}{|l|}{$\mathrm{AlBr}$} \\
\hline$a^{3} \Pi$ & $-4,2$ & 15,7 & $-3,6$ & $-33,6$ & $-7,9$ & $-28,9$ & $-62,6$ & $-62,7$ \\
\hline $\mathrm{A}^{1} \Pi$ & $-18,4$ & 213,8 & 135,5 & $-102,9$ & 39,8 & $-362,6$ & $-94,8$ & $-95,1$ \\
\hline \multicolumn{9}{|l|}{$\mathrm{BF}$} \\
\hline$a^{3} \Pi$ & $-13,4$ & 14,0 & 49,8 & $-43,0$ & 77,6 & $-52,9$ & 32,1 & 33,3 \\
\hline $\mathrm{A}^{1} \Pi$ & $-10,3$ & 86,0 & 96,3 & $-150,6$ & 130,4 & $-185,1$ & $-33,4$ & $-33,8$ \\
\hline \multicolumn{9}{|l|}{$\mathrm{BCl}$} \\
\hline$a^{3} \Pi$ & $-9,5$ & 60,8 & $-56,1$ & $-72,9$ & $-58,8$ & $-58,2$ & $-194,7$ & $-194,7$ \\
\hline $\mathrm{A}^{1} \Pi$ & $-15,4$ & 417,3 & 152,1 & $-242,0$ & 58,9 & $-604,9$ & $-234,0$ & $-234,2$ \\
\hline \multicolumn{9}{|l|}{$\mathrm{BBr}$} \\
\hline$a^{3} \Pi$ & $-8,8$ & $-13,0$ & $-269,1$ & 22,1 & $-148,2$ & 224,7 & $-192,3$ & $-192,2$ \\
\hline $\mathrm{A}^{1} \Pi$ & $-22,4$ & 447,6 & 102,8 & $-81,0$ & $-48,0$ & $-603,9$ & $-204,9$ & $-204,8$ \\
\hline \multicolumn{9}{|l|}{$\mathrm{CS}$} \\
\hline$a^{3} \Pi$ & $-27,0$ & $-860,1$ & 158,7 & 314,9 & $-121,5$ & 444,9 & $-90,1$ & $-90,1$ \\
\hline $\mathrm{A}^{1} \Pi$ & $-27,8$ & $-364,7$ & 814,9 & 262,6 & $-40,1$ & $-731,9$ & $-86,9$ & $-87,0$ \\
\hline
\end{tabular}

${ }^{\mathrm{a}} \triangle A=A$ (estado excitado $)-A($ estado fundamental).

Para a maioria dos sistemas considerados, exceto para o CS, as variações das intensidades de infravermelho durante a excitação eletrônica podem ser explicadas por mudanças na contribuição de carga, juntamente com as variações nos termos cruzados entre a carga e os fluxos de carga e de dipolo atômicos $\left(\Delta A^{C}+\Delta A^{C \times C F}+\Delta A^{C \times D F}\right)$, como mostrado na Figura 19, o que indica um padrão similar ao encontrado para a molécula de CO (TERRABUIO et al., 2017). O caso da molécula de CS é inesperado e a interpretação para a 
redução das intensidades desta molécula é bem mais complicada. Neste caso e também na molécula de $\mathrm{AlH}$, a soma das três contribuições restantes, $\Delta A^{C F}+\Delta A^{D F}+\Delta A^{C F \times D F}$, assume valores surpreendentemente elevados (ao redor de $366 \mathrm{~km} \mathrm{~mol}^{-1} \mathrm{AlH}$ e de $-269 \mathrm{~km} \mathrm{~mol}^{-1}$ para CS). A razão para este comportamento anômalo ainda precisa ser melhor investigada.

Figura 19 - Variação da intensidade de infravermelho dos modos de estiramento das moléculas $\mathrm{AlH}, \mathrm{AlF}, \mathrm{AlCl}, \mathrm{AlBr}, \mathrm{BF}, \mathrm{BCl}, \mathrm{BBr}$ durante a excitação eletrônica, $\triangle A$, em função da soma da contribuição de carga, $\Delta \boldsymbol{A}^{\boldsymbol{C}}$, e seus termos cruzados com fluxo de carga e fluxo de dipolo, $\Delta \boldsymbol{A}^{\mathbf{C} \times \mathbf{C F}}$ e $\Delta \boldsymbol{A}^{\mathbf{C} \times \mathbf{D F}}\left(\mathrm{em} \mathrm{km} \mathrm{mol}^{-1}\right)$

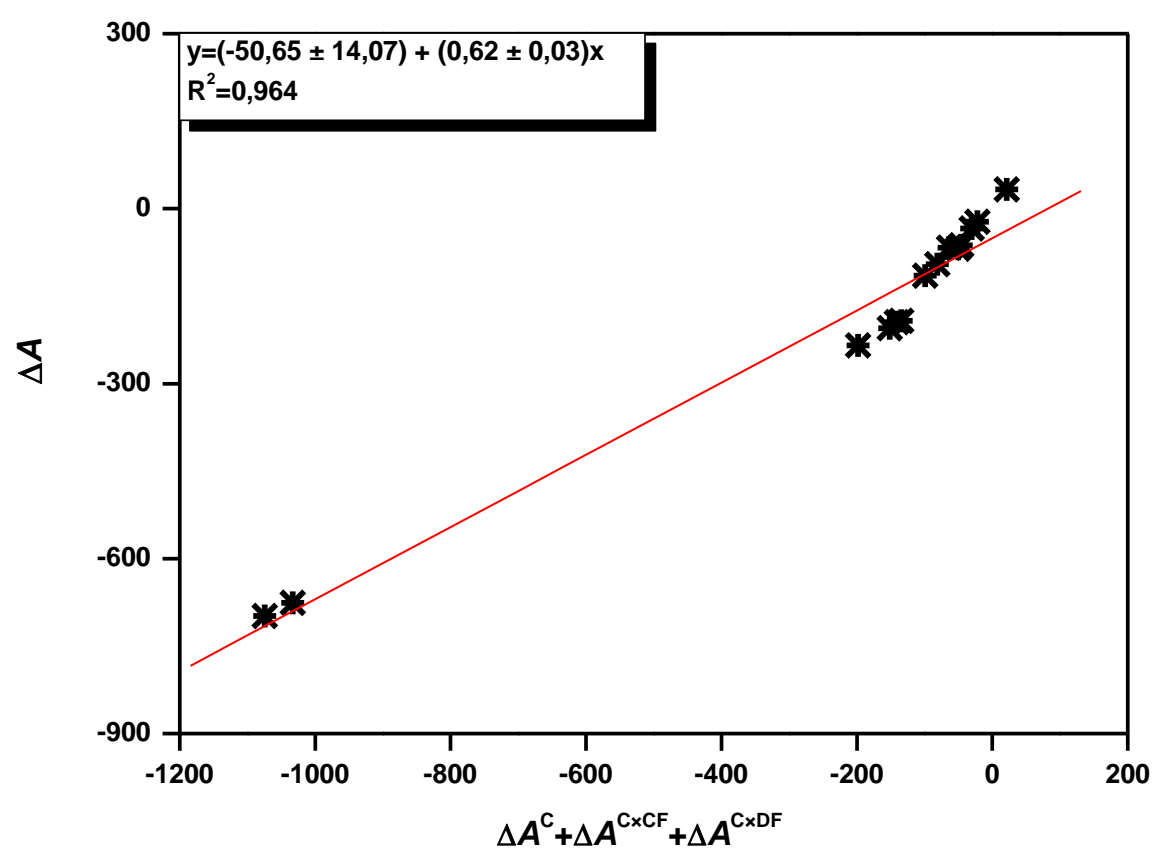

\section{CONCLUSÕES}

A transição eletrônica $\sigma \rightarrow \pi^{*}$ acarreta uma diminuição na magnitude do momento de dipolo para os estados excitados das moléculas $\mathrm{BF}, \mathrm{BCl}, \mathrm{BBr}$ e $\mathrm{CS}$ e, além disso, observa-se uma inversão no sentido do vetor para os respectivos estados tripletos. Estas mudanças podem ser explicadas principalmente pela diminuição expressiva das contribuições de polarização atômica do boro e do carbono durante as excitações, as quais são mais pronunciadas para o estado $\mathrm{T}_{1}$ do que para o estado $\mathrm{S}_{1}$. Para o $\mathrm{AlH}$, a excitação para ambos os estados produz intensificação do momento de dipolo, enquanto o oposto ocorre para AlBr. Nos demais sistemas ( $\mathrm{AlF}$ e $\mathrm{AlCl}$ ), o momento de dipolo se intensifica para os estados tripletos e se enfraquece para os estados excitados singletos, porém o vetor continua com o mesmo sentido observado nos respectivos estados fundamentais. A explicação para as variações de momento de dipolo detectadas no AlF durante as transições eletrônicas depende muito do que ocorre com a contribuição AP do alumínio, enquanto as separações de carga muito distintas 
observadas nos estados $S_{1}$ e $T_{1}$ constituem a principal explicação para as mudanças de momento de dipolo do $\mathrm{AlH}, \mathrm{AlCl}$ e $\mathrm{AlBr}$.

Em geral, com relação às propriedades topológicas, as excitações verticais ocasionam reduções na densidade eletrônica e na magnitude da densidade de energia total nos BCPs dos sistemas estudados. Por sua vez, o Laplaciano da densidade eletrônica nestes BCPs tende a diminuir para os sistemas contendo alumínio e aumentar para aqueles com boro. No que se referem às propriedades espectroscópicas, observa-se um deslocamento batocrômico das frequências vibracionais do BF e do CS em ambas as transições eletrônicas, enquanto o oposto, deslocamento hipsocrômico, é encontrado no $\mathrm{BCl}$ e $\mathrm{BBr}$. Nos demais sistemas, $\mathrm{AlH}$, $\mathrm{AlF}, \mathrm{AlCl}$ e AlBr, ocorrem deslocamento batocrômicos no processo $\mathrm{S}_{0} \rightarrow \mathrm{S}_{1}$ e deslocamentos hipsocrômicos nos processos $\mathrm{S}_{0} \rightarrow \mathrm{T}_{1}$. Além disso, as bandas vibracionais no infravermelho quase sempre se tornam menos intensas com a excitação eletrônica (exceto pelo BF) e estas variações de intensidade são explicadas devido às mudanças da contribuição de carga, juntamente com as variações nos termos cruzados entre a carga e os fluxos de carga e de dipolo atômicos, $\left(\Delta A^{C}+\Delta A^{C \times C F}+\Delta A^{C \times D F}\right)$ (exceto para o CS).

\section{PERSPECTIVAS}

Investigar as variações das propriedades da densidade eletrônica e das intensidades de infravermelho, segundo modelo CCFDF/QTAIM, durante a excitação eletrônica em sistemas mais variados (diferentes famílias de moléculas e casos poliatômicos). Entre estes, seria possível citar ainda casos exóticos, como sistemas que contenham atratores não-nucleares. A principal dúvida a ser respondida aqui é se a excitação eletrônica é capaz de eliminar o atrator ou não.

\section{REFERÊNCIAS}

ADAMSON, A. W. Properties of excited states. Journal of Chemical Education, v. 60, p. 797-802, 1983.

BADER, R. F. W. Atoms in Molecules: A Quantum Theory. $1^{\text {a }}$ ed. Oxford, UK: Clarendon Press, 1990.

COYLE, J. D. Introduction to Organic Photochemistry, England: Wiley, 2004.

DEMAS, J. N. Photophysical pathways in metal complexes, Journal of Chemical Education, 
v. 60, p. $803-808,1983$.

DUNNING JR, T. H. Gaussian basis sets for use in correlated molecular calculations. I. The atoms boron through neon and hydrogen. The Journal of Chemical Physics, v. 90, p. 1007$1023,1989$.

FERRO-COSTAS, D.; PENDÁS, A. M.; GONZÁLEZ, L. e MOSQUERA, R. A. Beyond the molecular orbital conception of electronically excited states through the quantum theory of atoms in molecules. Physical Chemistry Chemical Physics, v. 16, p. 9249-9258, 2014.

FRISCH, M. J.; TRUCKS G. W.; SCHLEGEL, H. B.; SCUSERIA, G. E.; ROBB, M. A.; CHEESEMAN, J. R.; SCALMANI, G.; BARONE, V.; MENNUCCI, B.; PETERSSON, G. A.; NAKATSUJI, H.; CARICATO, M.; LI, X.; HRATCHIAN, H. P.; IZMAYLOV, A. F.; BLOINO, J.; ZHENG, G.; SONNENBERG, J. L.; HADA, M.; EHARA, M.; TOYOTA, K.; FUKUDA, R.; HASEGAWA, J.; ISHIDA, M.; NAKAJIMA, T.; HONDA, Y.; KITAO, O.; NAKAI, H.; VREVEN, T.; MONTGOMERY JR., J. A.; PERALTA, J. E.; OGLIARO, F.; BEARPARK, M.; HEYD, J. J.; BROTHERS, E.; KUDIN, K. N.; STAROVEROV, V. N.; KEITH, T.; KOBAYASHI, R.; NORMAND, J.; RAGHAVACHARI, K.; RENDELL, A.; BURANT, J. C.; IYENGAR, S. S.; TOMASI, J.; COSSI, M.; REGA, N.; MILLAM, J. M.; KLENE, M.; KNOX, J. E.; CROSS, J. B.; BAKKEN, V.; ADAMO, C.; JARAMILLO, J.; GOMPERTS, R.; STRATMANN, R. E.; YAZYEV, O.; AUSTIN, A. J.; CAMMI, R.; POMELLI, C.; OCHTERSKI, J. W.; MARTIN, R. L.; MOROKUMA, K.; ZAKRZEWSKI, V. G.; VOTH, G. A.; SALVADOR, P.; DANNENBERG, J. J.; DAPPRICH, S.; DANIELS, A. D.; FARKAS, O.; FORESMAN, J. B.; ORTIZ, J. V.; CIOSLOWSKI, J. e FOX, D. J. Gaussian 09, Revision D.01, Gaussian Inc., Wallingford, CT, 2013.

GARCÍA-REVILLA, M. A.; CORTÉZ-GUZMÁN, F.; ROCHA-RINZA, T. e HERNANDÉZTRUJILLO, J. Latin American contributions to quantum chemical topology. International Journal of Quantum Chemistry, v. 119, p. 3-26, 2019.

GUTIÉRREZ-ARZALUZ, L.; ROCHA-RINZA, T. e CORTÉS-GUZMÁN, F. Stilbene photoisomerization driving force as revealed by the topology of the electron density and QTAIM properties. Computational and Theoretical Chemistry, v. 1053, p. 214-2019, 2015.

HAIDUKE, R. L. A. e BRUNS, R. E. An atomic charge-charge flux-dipole flux atom-inmolecule decomposition for molecular dipole-moment derivatives and infrared fundamental intensities. Journal of Chemical Physics A, v. 109, p. 2680-2688, 2005.

HAYNES, W. M. Ed. CRC Handbook of Chemistry e Physics; 96th ed. CRC Press, Taylor e Francis: Boca Raton, FL, 2016.

JACQUEMIN, D.; WATHELET, V.; PERPÈTE, E. A. e ADAMO, C. Extensive TD-DFT benchmark: singlet-excited states of organic molecules. Journal of Chemical Theory and Computation, v. 5, p. 2420-2435, 2009.

JARA-CORTÉS, J.; ROCHA-RINZA, T. e HERNÁNDEZ-TRUJILLO, J. Electron density analysis of aromatic complexes in excited electronic states: The benzene and naphthalene excimers. Computational and Theoretical Chemistry, v. 1053, p. 220-228, 2015.

KENDALL, R. A.; DUNNING JR., T.H. e HARRISON, R. J. Electron affinities of the 
first-row atoms revisited. Systematic basis sets and wave functions. The Journal of Chemical Physics, v. 96, p. 6796-6806, 1992.

KEITH, T. A. AIMAll (version 16.10.31), TK Gristmill Software, Overland Park KS, USA, 2013.

LAKOWICZ, J. R. Principles of Fluorescence Spectroscopy, 3rd ed., Springer: USA, 2010.

LEANG, S. S., ZAHARIEV, F. e GORDON, M.S. Benchmarking the performance of timedependent density functional methods. The Journal of Chemical Physics. v. 136, p.1-12, 2012.

MARTINS, G. R. e DE OLIVEIRA, A. E. Low-Lying electronic excited states and fundamental infrared intensities for ozone: the QTAIM/CCFDF model. Journal of Molecular Structure, v. 1150, p. 21-27, 2017.

MATTA, C. F. e BOYD, R. J. The Quantum Theory of Atoms in Molecules From Solid State to DNA and Drug Design, Weinheim: Wiley-Vch, 2007. 1-21 p.

NATIONAL INSTITUTE OF STANDARDS E TECHNOLOGY. Chemistry webbook. U. S. Secretary of Commerce: the standard reference data act. 1968. Disponível em: <http//www.nist.gov/index.html>. Acesso em: 05 dez. 2017.

SERRANO-ANDRÉS, L. e MERCHÁN, M. Quantum Chemistry of the excited state: 2005 overview. Journal of Molecular Structure, v. 729, p. 99-108, 2005.

TERRABUIO, L. A.; Da SILVA, N. A.; HAIDUKE, R. L. A. e MATTA, C. F. Real space atomic decomposition of fundamental properties of carbon monoxide in the ground and the two lowest lying excited electronic states. Molecular Physics, v. 115, p. 1955-1965, 2017.

TERRABUIO, L. A.; RICHTER, W. E.; SILVA, A. F.; BRUNS, R. E. e HAIDUKE, R. L. A. An atom in molecules study of infrared intensity enhancements in fundamental donor stretching bands in hydrogen bond formation. Physical Chemistry Chemical Physics, v. 16, p. 24920-24928, 2014.

WILSON, A. K.; WOON, D. E.; PETERSON, K. A. e DUNNING JR, T. H. Gaussian basis sets for use in correlated molecular calculations. IX. The atoms gallium through krypton.

Journal of Chemical Physics, v. 110, 7667-7676, 1999.

WOON, D. E. e DUNNING Jr., T. H. Gaussian-basis sets for use in correlated molecular calculations. 3. The atoms aluminum through argon. The Journal of Chemical Physics, v. 98, p. 1358-1371, 1993. 
Capítulo 5

Propriedades topológicas da densidade eletrônica para a predição da reatividade e da regiosseletividade em substituições eletrofílicas aromáticas 


\section{INTRODUÇÃO}

As reações de substituição eletrofílica aromática $\left(\mathrm{S}_{\mathrm{E}} \mathrm{Ar}\right)$ são processos fundamentais na química orgânica, pois tanto o composto aromático quanto o eletrófilo $\left(\mathrm{Y}^{+}\right)$estão sujeitos a ampla variação, resultando na síntese de inúmeros compostos com vasta aplicabilidade (BRUICE, 2004; CLAIDEN, GREEVES e WARREN, 2012; LIU, 2014; McMURRY, 2007). As reações representativas de $S_{E} A r$ do benzeno são nitração, sulfonação, halogenação, alquilação e acilação de Friedel Crafts, sendo que todas elas ocorrem pelo mesmo tipo de mecanismo em duas etapas e diferem apenas no modo como o eletrófilo necessário para iniciar a reação é gerado (BRUICE, 2004; McMURRY, 2007). Se representarmos um areno pela fórmula geral ArH, em que Ar é o grupo arila, a parte eletrofílica do composto genérico Y-B substitui um dos hidrogênios do anel, ou seja,

$$
\begin{gathered}
\boldsymbol{\delta}+\boldsymbol{\delta}- \\
\mathrm{Ar}-\mathbf{H}+\mathbf{Y}-\mathrm{B} \rightarrow \mathrm{Ar}-\mathbf{Y}+\mathbf{H}-\mathrm{B} .
\end{gathered}
$$

Os princípios mecanísticos destas reações, ilustrados na Figura 20, envolvem a utilização de um par de elétrons $\pi$ do benzeno para formar uma ligação com o eletrófilo $\left(\mathrm{Y}^{+}\right)$, produzindo um carbocátion na primeira etapa (BRUICE, 2004; McMURRY, 2007). A formação deste intermediário é relativamente lenta e endergônica porque uma substância aromática está sendo convertida em um composto não aromático menos estável (BRUICE, 2004). Após a sua formação, o íon arênio perde rapidamente um próton, restaurando a aromaticidade do anel e resultando no produto da substituição eletrofílica aromática. Esta segunda etapa é rápida e fortemente exergônica porque recupera a aromaticidade, aumentando a estabilidade (CLAIDEN, GREEVES e WARREN, 2012). 
Figura 20 - Diagrama de coordenada de reação para a substituição eletrofílica do benzeno

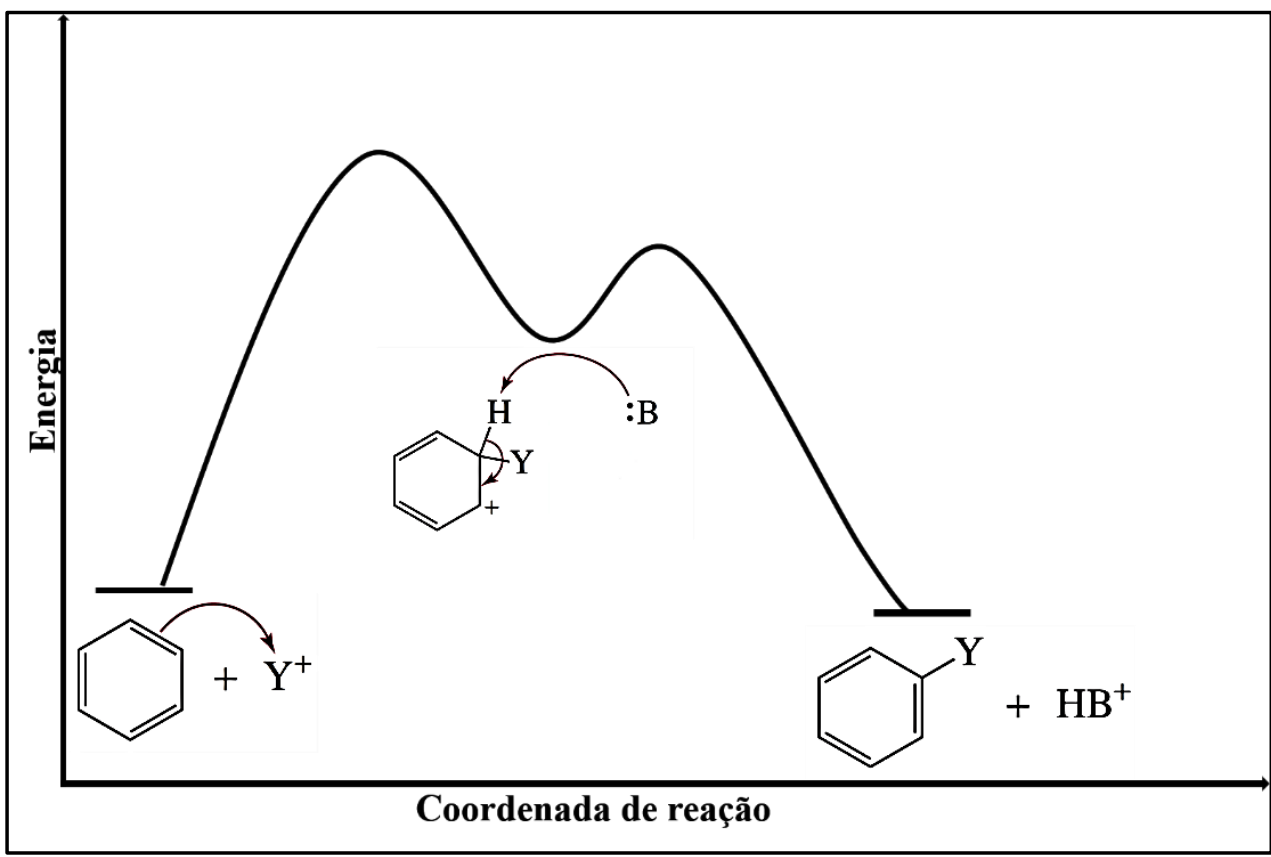

FONTE: Adaptado de BRUICE, P. Y. Organic Chemistry. Upper Saddle River: Prentice Hall, 2004.

A capacidade de compostos aromáticos de interagirem com uma diversidade de doadores e aceptores de elétrons é modulada por conta da presença de substituintes, que modificam a natureza eletrônica do benzeno, e isto produz grande impacto em diversas áreas da química, como a bioquímica e a ciência dos materiais, porque leva a um comportamento não homogêneo dos carbonos do anel (BAUZÁ et al., 2013). A análise da substituição em anéis que já tenham pelo menos um substituinte suscita diversos questionamentos sobre os fatores que governam estas reações, tais como: (1) Qual é o efeito de um substituinte na reatividade e regiosseletividade do anel aromático em reações de substituição eletrofílica? (2) Quais descritores seriam apropriados para predizer os sítios reativos em processos de $\mathrm{S}_{\mathrm{E}} \mathrm{Ar}$ ? e (3) Como a reatividade e a regiosseletividade podem ser associadas com a distribuição espacial de carga eletrônica do composto aromático inicial (KOLEVA et al., 2009)?

Grupos ativadores, como $-\mathrm{NH}_{2},-\mathrm{OH}$ e $-\mathrm{OCH}_{3}$, tornam o anel benzênico mais reativo diante da substituição eletrofílica, enquanto os desativadores, como $-\mathrm{F},-\mathrm{Cl},-\mathrm{Br}$ e $-\mathrm{CN}$, o tornam menos reativo (CLAIDEN, GREEVES e WARREN, 2012). De acordo com a expectativa experimental, todos os substituintes fortemente ativantes doam elétrons ao anel por ressonância e retiram elétrons do anel indutivamente, enquanto os fortemente desativantes são somente retiradores de elétrons (BRUICE, 2004). Além disso, os ativadores ou desativadores previamente ligados ao benzeno determinam a posição de um novo grupo. 
Todos os substituintes ativantes e os halogênios, fracamente desativantes, são chamados de orientadores orto/para, enquanto todos os substituintes mais desativadores que os halogênios são orientadores meta (BRUICE, 2004; CLAIDEN, GREEVES e WARREN, 2012; McMURRY, 2007). A princípio, com base nestas informações, seria esperado que uma análise de carga eletrônica fornecesse a conexão entre a estrutura eletrônica de arenos e sua reatividade em processos de $\mathrm{S}_{\mathrm{E}} \mathrm{Ar}$. Todavia, os resultados sugerem que a habilidade de substituintes para ativar ou desativar o anel aromático não pode ser associada com a carga total do anel ou com as cargas parciais dos átomos constituintes em virtude do fato de que há uma relação mais complexa entre a reatividade e a população eletrônica $\pi$ (BADER e CHANG, 1989). Além disto, a contribuição da população eletrônica $\sigma$ causa problemas nestes tipos de análise, pois a reatividade deveria depender quase exclusivamente da população $\pi$ durante a etapa lenta de formação do íon arênio, e, normalmente, não é fácil separar ambas as contribuições para a população total de cada carbono.

A ideia principal dos descritores estáticos de reatividade seria que as informações obtidas das características dos reagentes isolados são suficientes para predizer o resultado da reação (FIEVEZ, 2011). Um índice simples de reatividade teórico é atraente, não apenas porque pode ser facilmente calculado, mas também porque pode fornecer informações químicas gerais. Vários índices de reatividade para predizer a regiosseletividade da reação $\mathrm{S}_{\mathrm{E}} \mathrm{Ar}$ foram propostos, por exemplo, Politzer et al. (1984) utilizaram o potencial eletrostático molecular de benzenos substituídos para a caracterização da ativação e desativação de anéis aromáticos durante o ataque eletrofílico e Zhou e Parr (1990) aplicaram o conceito de dureza no estudo da regiosseletividade da substituição eletrofílica aromática. Outro exemplo é dado pela afinidade eletrofílica que, no entanto, se baseia na observação experimental de que a estabilidade do complexo $\sigma$ reflete o resultado da reação, de modo que seria classificado como um índice mais a posteriori do que a priori (FIEVEZ et al., 2011)

Além disso, as propriedades advindas da Teoria Quântica de Átomos em Moléculas (QTAIM) (BADER, 1990) oferecem outros meios para se tentar predizer os sítios de ataques eletrofílicos em reações de substituição de compostos aromáticos. Bader e Chang (1989) mostraram que a habilidade de um substituinte em ativar ou desativar o anel aromático, isto é, torná-lo mais ou menos reativo, é refletida pela população $\pi$ e momentos de quadrupolo atômicos dos carbonos do anel. Se o eixo $z$ do sistema de coordenadas Cartesiano está centrado no núcleo de um átomo do sistema aromático, então o componente $z z$ do momento de quadrupolo da densidade de carga eletrônica para o átomo $\Omega$ é (MATTA e BOYD, 2007): 


$$
Q_{Z Z}(\Omega)=-e \int_{\Omega}\left(3 z_{\Omega}^{2}-\vec{r}_{\Omega}^{2}\right) \rho(\vec{r}) d \vec{r}
$$

em que $\rho$ é a densidade eletrônica no ponto $\vec{r}\left(x_{\Omega}, y_{\Omega}\right.$ e $\left.z_{\Omega}\right)$ e $e$ é a carga elementar do elétron.

O momento de quadrupolo é uma medida do desvio da nuvem eletrônica do átomo de uma simetria esférica (MATTA e BOYD, 2007; BADER, 1990; BADER e CHANG, 1989). Um valor negativo para $Q_{z z}$ implica que a esfera está distorcida ao longo do eixo $z$, enquanto um valor positivo desta propriedade resulta em uma distorção da esfera no plano $x y$ (MOA e MOSKERA, 2006; CORMIER, WATT e LEWIS, 2010). A aproximação do eletrófilo em reações de $S_{E} A r$ deveria ser favorecida por uma menor concentração de carga eletrônica no plano do anel (definido como plano xy) e uma maior concentração desta carga ao longo do eixo perpendicular ao plano $x y$ devido a presença de elétrons $\pi$. Deste modo, substituintes ativantes deveriam produzir um valor menor de $Q_{z z}$ em átomos de carbono que aqueles do benzeno, enquanto substituintes desativantes deveriam estar associados com um valor maior de $Q_{Z Z}$ que os do benzeno (MOA e MOSKERA, 2006; BADER e CHANG, 1989).

Geralmente, o entendimento dos processos $S_{E} A r$ requer a elucidação da relação entre a estrutura eletrônica do benzeno e sua reatividade em diferentes posições do anel. Neste sentido, este trabalho objetiva expandir o estudo de Bader e Chang (1989) e investigar a influência de um conjunto maior de substituintes, considerados experimentalmente como fortemente ativantes $\left(-\mathrm{NH}_{2},-\mathrm{NHCH}_{3},-\mathrm{N}\left(\mathrm{CH}_{3}\right)_{2},-\mathrm{OH}\right.$ e $\left.-\mathrm{OCH}_{3}\right)$, fracamente ativante ($\left.\mathrm{CHCH}_{2}\right)$, fracamente desativantes $(-\mathrm{F},-\mathrm{Cl}$ e $-\mathrm{Br})$, moderadamente desativantes $(-\mathrm{COH}$, $\mathrm{COCH}_{3},-\mathrm{COOCH}_{3},-\mathrm{COOH}$ e $-\mathrm{COCl}$ ) e fortemente desativantes (-CN e $-\mathrm{NO}_{2}$ ), nos momentos de quadrupolo atômico do anel aromático usando um nível de cálculo mais avançado do que aquele escolhido no estudo precursor e, assim, predizer a reatividade e os sítios de ataques eletrofílicos em reações de $S_{E} A r$.

Além disso, outro objetivo seria propor propriedades topológicas alternativas, derivadas da QTAIM, como descritores de ativação/desativação e dos efeitos de grupos dirigentes na suscetibilidade das posições orto, meta e para perante reações de $\mathrm{S}_{\mathrm{E}} \mathrm{Ar}$. Por fim, também introduzimos quantidades energéticas derivadas da partição de Átomos Quânticos Interagentes (PENDÁS, BLANCO e FRANCISCO, 2005 e 2006) na predição da reatividade e da regiosseletividade em compostos aromáticos substituídos. 


\section{OBJETIVO}

2.1 Investigar quantidades topológicas alternativas advindas da QTAIM, como densidades de energia cinética e potencial $\left(G_{R C P}\right.$ e $\left.V_{R C P}\right)$ e curvaturas da densidade eletrônica $(\lambda)$ nos pontos críticos dos anéis aromáticos (RCPs) e nos atratores nucleares dos átomos de carbono (NCP), bem como a energia intra-atômica $\left(\Delta E_{\text {Intra }}\right)$, obtida segundo a partição de Átomos Quânticos Interagentes, como descritores para predizer a reatividade e regiosseletividade em reações de substituição eletrofílica aromática baseando-se apenas nas propriedades dos substratos iniciais.

\section{METODOLOGIA}

Os cálculos de estrutura eletrônica foram realizados no programa Gaussian 09 (FRISCH et al., 2009) utilizando-se o funcional duplo híbrido B2PLYP (GRIMME, 2006) com o conjunto de funções de base cc-pVQZ (DUNNING JR, 1989; WILSON et al.; 1999; WOON e DUNNING JR, 1992). Primeiramente, as geometrias de equilíbrio foram encontradas. Na sequência, os quadrupolos atômicos e as propriedades topológicas nos pontos críticos do anel (curvaturas da densidade eletrônica e densidades de energia cinética e potencial) foram obtidos no programa AIMAll (KEITH, 2013), por meio das densidades eletrônicas previamente determinadas nas geometrias de equilíbrio. As curvaturas da densidade eletrônica perpendiculares aos atratores nucleares de carbono também foram avaliadas. Além disso, avaliou-se o efeito da substituição do hidrogênio por grupos ativadores e desativadores na energia intra-atômica dada pelo formalismo de Átomos Quânticos Interagentes (IQA) (BLANCO, PENDÁS e FRANCISCO, 2005). Esta análise foi realizada empregando-se densidades eletrônicas determinadas em nível B3LYP/cc-pVQZ, a partir das geometrias otimizadas com B2PLYP/cc-pVQZ, o que foi necessário em virtude da alta demanda por recursos computacionais que seria requerida no caso de usar densidades fornecidas pelo funcional duplo híbrido no formalismo IQA. 


\section{RESULTADOS E DISCUSSÕES}

\subsection{Efeitos dos substituintes nos momentos de quadrupolo dos átomos de carbono de anéis aromáticos}

Os substituintes investigados neste trabalho são classificados de acordo com o modo como afetam experimentalmente a reatividade do anel benzênico diante da substituição eletrofílica em compostos aromáticos, quando comparados com o benzeno, no qual o substituinte é o hidrogênio (Quadro 1).

Quadro 1 - Efeitos de substituintes na reatividade do anel benzênico perante reações de substituição eletrofílica

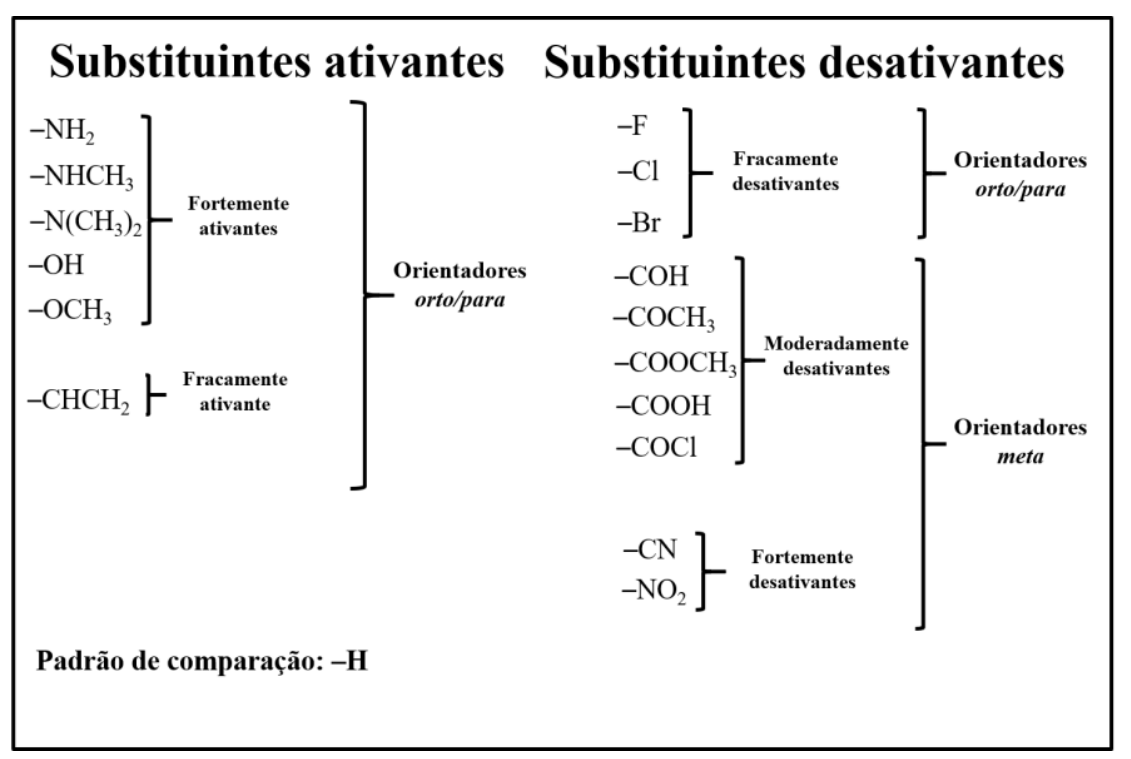

FONTE: Adaptado de BRUICE, P. Y. Organic Chemistry. Upper Saddle River: Prentice Hall, 2004.

A Tabela 29 mostra as variações dos componentes $z z$ dos momentos de quadrupolo dos átomos de carbono dos compostos aromáticos substituídos em relação ao benzeno $\left(\Delta Q_{z z}\right.$, sendo $z$ o eixo perpendicular ao plano do anel benzênico). Como esperado, em termos dos quadrupolos atômicos, $Q_{z z}$, nota-se que os substituintes ativantes $\left(-\mathrm{NH}_{2},-\mathrm{NHCH}_{3},-\mathrm{N}\left(\mathrm{CH}_{3}\right)_{2}\right.$, $-\mathrm{OH},-\mathrm{OCH}_{3}$ e $-\mathrm{CHCH}_{2}$ ) fazem com que valores mais negativos desta propriedade sejam observados em certas posições, quando comparados aos resultados do benzeno, o que indica uma distorção da nuvem eletrônica ao longo do eixo $z$. Desta forma, isto favorece a aproximação no eletrófilo, para dar origem ao intermediário da reação. Por sua vez, os 
substituintes desativantes $\left(-\mathrm{F},-\mathrm{Cl},-\mathrm{Br},-\mathrm{COH},-\mathrm{COCH}_{3},-\mathrm{COOCH}_{3},-\mathrm{COOH},-\mathrm{COCl},-\mathrm{CN}\right.$ e $-\mathrm{NO}_{2}$ ) dão origem a valores mais positivos de $Q_{z z}$, exceto pelo flúor, cloro e $-\mathrm{COCH}_{3}$.

Tabela 29 - Variações nos componentes $z z$ dos momentos de quadrupolo atômicos, $\Delta Q_{z z}$, do carbono de compostos aromáticos substituídos (ArY, em que Y é o substituinte) em relação ao benzeno (Ar), segundo cálculos B2PLYP/cc-pVQZ (em u. a.)

\begin{tabular}{|c|c|c|c|c|c|}
\hline Substituinte (Y) & Átomo & $\Delta Q_{\mathrm{ZZ}}{ }^{a}$ & Substituinte $(\mathrm{Y})$ & Átomo & $\Delta Q_{\mathrm{ZZ}}$ \\
\hline \multirow[t]{6}{*}{$-\mathrm{NH}_{2}$} & $\mathrm{C}$ & 0,442 & $-\mathrm{Br}$ & $\mathrm{C}$ & 0,331 \\
\hline & $\mathrm{C}_{\text {orto }}$ & $-0,328$ & & $\mathrm{C}_{\text {orto }}$ & 0,071 \\
\hline & $\mathrm{C}_{\text {Meta }}$ & 0,080 & & $\mathrm{C}_{\text {Meta }}$ & 0,077 \\
\hline & $\mathrm{C}_{\text {Para }}$ & $-0,244$ & & $\mathrm{C}_{\text {Para }}$ & 0,010 \\
\hline & $\mathrm{C}_{\text {Meta }}$ & 0,081 & & $\mathrm{C}_{\text {Meta }}$ & 0,077 \\
\hline & $\mathrm{C}_{\text {Orto }}$ & $-0,326$ & & $\mathrm{C}_{\text {Orto }}$ & 0,071 \\
\hline \multirow[t]{6}{*}{$-\mathrm{NHCH}_{3}$} & $\mathrm{C}$ & 0,445 & $-\mathrm{COH}$ & $\mathrm{C}$ & 0,009 \\
\hline & Corto & $-0,420$ & & $\mathrm{C}_{\text {orto }}$ & 0,127 \\
\hline & $\mathrm{C}_{\text {Meta }}$ & 0,077 & & $\mathrm{C}_{\text {Meta }}$ & 0,017 \\
\hline & $\mathrm{C}_{\text {Para }}$ & $-0,258$ & & $\mathrm{C}_{\text {Para }}$ & 0,157 \\
\hline & $\mathrm{C}_{\text {Meta }}$ & 0,079 & & $\mathrm{C}_{\text {Meta }}$ & 0,050 \\
\hline & $\mathrm{C}_{\text {Orto }}$ & $-0,330$ & & $\mathrm{C}_{\text {Orto }}$ & 0,241 \\
\hline \multirow[t]{6}{*}{$-\mathrm{N}\left(\mathrm{CH}_{3}\right)_{2}$} & $\mathrm{C}$ & 0,372 & $-\mathrm{COCH}_{3}$ & $\mathrm{C}$ & $-0,010$ \\
\hline & $\mathrm{C}_{\text {Orto }}$ & $-0,427$ & & $\mathrm{C}_{\text {Orto }}$ & 0,229 \\
\hline & $\mathrm{C}_{\text {Meta }}$ & 0,053 & & $\mathrm{C}_{\text {Meta }}$ & 0,029 \\
\hline & $\mathrm{C}_{\text {Para }}$ & $-0,269$ & & $\mathrm{C}_{\text {Para }}$ & 0,126 \\
\hline & $\mathrm{C}_{\text {Meta }}$ & 0,053 & & $\mathrm{C}_{\text {Meta }}$ & $-0,005$ \\
\hline & $\mathrm{C}_{\text {Orto }}$ & $-0,427$ & & $\mathrm{C}_{\text {Orto }}$ & 0,073 \\
\hline \multirow[t]{6}{*}{$-\mathrm{OH}$} & $\mathrm{C}$ & 0,371 & $-\mathrm{COOCH}_{3}$ & $\mathrm{C}$ & $-0,049$ \\
\hline & $\mathrm{C}_{\text {orto }}$ & $-0,195$ & & $\mathrm{C}_{\text {orto }}$ & 0,210 \\
\hline & $\mathrm{C}_{\text {Meta }}$ & 0,097 & & $\mathrm{C}_{\text {Meta }}$ & 0,015 \\
\hline & $\mathrm{C}_{\text {Para }}$ & $-0,184$ & & $\mathrm{C}_{\text {Para }}$ & 0,117 \\
\hline & $\mathrm{C}_{\text {Meta }}$ & 0,085 & & $\mathrm{C}_{\text {Meta }}$ & 0,003 \\
\hline & Corto & $-0,322$ & & $\mathrm{C}_{\text {Orto }}$ & 0,155 \\
\hline \multirow[t]{5}{*}{$-\mathrm{OCH}_{3}$} & $\mathrm{C}$ & 0,323 & $-\mathrm{COOH}$ & $\mathrm{C}$ & $-0,067$ \\
\hline & $\mathrm{C}_{\text {Orto }}$ & $-0,183$ & & $\mathrm{C}_{\text {Orto }}$ & 0,230 \\
\hline & $\mathrm{C}_{\text {Meta }}$ & 0,077 & & $\mathrm{C}_{\text {Meta }}$ & 0,027 \\
\hline & $\mathrm{C}_{\text {Para }}$ & $-0,184$ & & $\mathrm{C}_{\text {Para }}$ & 0,143 \\
\hline & $\mathrm{C}_{\text {Meta }}$ & 0,059 & & $\mathrm{C}_{\text {Meta }}$ & 0,016 \\
\hline
\end{tabular}




\begin{tabular}{|c|c|c|c|c|}
\hline Substituinte $(\mathrm{Y})$ & Átomo & $\Delta Q_{Z Z}{ }^{a}$ Substituinte $(\mathrm{Y})$ & Átomo & $\Delta Q_{Z Z}$ \\
\hline & $\mathrm{C}_{\text {Orto }}$ & $-0,372$ & $\mathrm{C}_{\text {Orto }}$ & 0,180 \\
\hline \multirow[t]{6}{*}{$-\mathrm{CHCH}_{2}$} & $\mathrm{C}$ & $0,229-\mathrm{COCl}$ & $\mathrm{C}$ & $-0,067$ \\
\hline & $\mathrm{C}_{\text {orto }}$ & $-0,032$ & $\mathrm{C}_{\text {orto }}$ & 0,261 \\
\hline & $\mathrm{C}_{\text {Meta }}$ & 0,013 & $\mathrm{C}_{\text {Meta }}$ & 0,059 \\
\hline & $\mathrm{C}_{\text {Para }}$ & $-0,004$ & $\mathrm{C}_{\text {Para }}$ & 0,211 \\
\hline & $\mathrm{C}_{\text {Meta }}$ & 0,023 & $\mathrm{C}_{\text {Meta }}$ & 0,045 \\
\hline & $\mathrm{C}_{\text {Orto }}$ & 0,002 & $\mathrm{C}_{\text {orto }}$ & 0,259 \\
\hline \multirow[t]{6}{*}{$-\mathrm{F}$} & $\mathrm{C}$ & $0,191-\mathrm{CN}$ & $\mathrm{C}$ & 0,082 \\
\hline & Corto & $-0,078$ & $\mathrm{C}_{\text {orto }}$ & 0,176 \\
\hline & $\mathrm{C}_{\text {Meta }}$ & 0,101 & $\mathrm{C}_{\text {Meta }}$ & 0,091 \\
\hline & $\mathrm{C}_{\text {Para }}$ & $-0,078$ & $\mathrm{C}_{\text {Para }}$ & 0,150 \\
\hline & $\mathrm{C}_{\text {Meta }}$ & 0,101 & $\mathrm{C}_{\text {Meta }}$ & 0,091 \\
\hline & $\mathrm{C}_{\text {Orto }}$ & $-0,079$ & $\mathrm{C}_{\text {Orto }}$ & 0,176 \\
\hline \multirow[t]{6}{*}{$-\mathrm{Cl}$} & $\mathrm{C}$ & $0,251-\mathrm{NO}_{2}$ & $\mathrm{C}$ & $-0,137$ \\
\hline & $\mathrm{C}_{\text {orto }}$ & 0,041 & $\mathrm{C}_{\text {orto }}$ & 0,260 \\
\hline & $\mathrm{C}_{\text {Meta }}$ & 0,080 & $\mathrm{C}_{\text {Meta }}$ & 0,085 \\
\hline & $\mathrm{C}_{\text {Para }}$ & $-0,006$ & $\mathrm{C}_{\text {Para }}$ & 0,183 \\
\hline & $\mathrm{C}_{\text {Meta }}$ & 0,081 & $\mathrm{C}_{\text {Meta }}$ & 0,085 \\
\hline & $\mathrm{C}_{\text {orto }}$ & 0,041 & $\mathrm{C}_{\text {orto }}$ & 0,259 \\
\hline
\end{tabular}

${ }^{\mathrm{a}} \Delta Q_{\mathrm{ZZ}}(\Omega)=Q_{\mathrm{ZZ}}(\Omega)_{\mathrm{ArY}}-Q_{\mathrm{ZZ}}(\Omega)_{\mathrm{Ar}}$.

Além disto, substituintes ativantes $\left(-\mathrm{NH}_{2},-\mathrm{NHCH}_{3},-\mathrm{N}\left(\mathrm{CH}_{3}\right)_{2},-\mathrm{OH},-\mathrm{OCH}_{3}\right.$ e $\mathrm{CHCH}_{2}$ ) sempre fornecem valores negativos de $\Delta Q_{z z}$ nas posições orto/para, juntamente com valores positivos de $\Delta Q_{z z}$ nas posições meta. Por sua vez, os substituintes fracamente desativantes $(-\mathrm{F},-\mathrm{Cl}$ e $-\mathrm{Br})$ apresentam valores negativos para as posições orto/para quando o substituinte é o flúor ou dão origem a valores positivos de $\Delta Q_{z z}$ em mais posições $(-\mathrm{Cl}$ e $\mathrm{Br}$ ), mas sempre mostrando a posição meta como aquela na qual $\Delta Q_{z z}$ é mais positivo. Isto significa que há uma menor concentração de carga eletrônica no eixo perpendicular ao plano do anel da posição meta comparativamente com as demais posições, favorecendo, assim, reações de substituição eletrofílica nas posições orto e para.

Por outro lado, os valores de $\Delta Q_{z z}$ dos carbonos das posições orto e para se tornam mais positivos com a presença de grupos moderadamente ou fortemente desativantes $(-\mathrm{COH}$, $-\mathrm{COCH}_{3},-\mathrm{COOCH}_{3},-\mathrm{COOH},-\mathrm{COCl},-\mathrm{CN}$ e $-\mathrm{NO}_{2}$ ) do que o observado para os átomos de 
carbono das posições meta. Nestes casos, a aproximação do eletrófilo aos carbonos meta é mais favorável quando comparada aos demais.

Em suma, mesmo quando consideramos vários substituintes de categorias distintas, o componente $z z$ do quadrupolo atômico dos átomos de carbono do anel benzênico continua se comportando como um excelente descritor de ativação/desativação (exceto principalmente pelo $-\mathrm{F}$ ) e, também, das orientações preferenciais para substituição eletrofílica (orto, meta ou para). Isto reforça as conclusões do trabalho prévio de Bader e Chang (1989).

\subsection{Propriedades topológicas QTAIM nos pontos críticos dos anéis aromáticos}

A Tabela 30 mostra as variações das propriedades topológicas, como densidade eletrônica $\left(\Delta \rho_{R C P}\right)$, Laplaciano da densidade eletrônica $\left(\Delta \nabla^{2} \rho_{R C P}\right)$ e suas curvaturas $(\Delta \lambda)$, densidades de energia cinética $\left(\triangle G_{R C P}\right)$ e potencial $\left(\triangle V_{R C P}\right)$ e densidade de energia total $\left(\triangle H_{R C P}\right)$ no ponto crítico do anel (RCP) dos compostos aromáticos substituídos em relação ao benzeno. Primeiramente, nota-se que a substituição do hidrogênio por grupos ativadores leva a uma diminuição da densidade eletrônica no RCP. Por outro lado, grupos desativadores promovem um aumento da concentração de densidade eletrônica no RCP, exceto pelos substituintes $-\mathrm{Fe}-\mathrm{CN}$.

Tabela 30 - Variações das propriedades topológicas densidade eletrônica $\left(\Delta \rho_{R C P}\right)$ e suas curvaturas $\left(\Delta \lambda_{1}, \Delta \lambda_{2}\right.$ e $\left.\Delta \lambda_{3}\right)$, Laplaciano da densidade eletrônica $\left(\Delta \nabla^{2} \rho_{R C P}\right)$, densidades de energia potencial e cinética $\left(\Delta V_{R C P}\right.$ e $\left.\Delta G_{R C P}\right)$, assim como a total $\left(\Delta H_{R C P}\right)$, no ponto crítico do anel aromático em relação ao benzeno (em u. a.)

\begin{tabular}{|c|c|c|c|c|c|c|c|c|}
\hline Substituinte & $\Delta \rho_{R C P}$ & $\Delta \lambda_{1}$ & $\Delta \lambda_{2}$ & $\Delta \lambda_{3}$ & $\Delta \nabla^{2} \rho_{R C P}$ & $\Delta V_{R C P}$ & $\Delta G_{R C P}$ & $\Delta H_{R C P}$ \\
\hline$-\mathrm{NH}_{2}$ & $-0,00030$ & 0,00086 & $-0,00568$ & 0,00271 & $-0,00211$ & 0,00042 & $-0,00047$ & $-0,00005$ \\
\hline$-\mathrm{NHCH}_{3}$ & $-0,00034$ & 0,00083 & $-0,00666$ & 0,00297 & $-0,00286$ & 0,00053 & $-0,00062$ & $-0,00009$ \\
\hline$-\mathrm{N}\left(\mathrm{CH}_{3}\right)_{2}$ & $-0,00038$ & 0,00076 & $-0,00885$ & 0,00444 & $-0,00365$ & 0,00064 & $-0,00078$ & $-0,00014$ \\
\hline$-\mathrm{OH}$ & $-0,00025$ & 0,00090 & $-0,00471$ & 0,00200 & $-0,00181$ & 0,00038 & $-0,00042$ & $-0,00004$ \\
\hline$-\mathrm{OCH}_{3}$ & $-0,00026$ & 0,00080 & $-0,00574$ & 0,00249 & $-0,00246$ & 0,00045 & $-0,00053$ & $-0,00008$ \\
\hline$-\mathrm{CHCH}_{2}$ & $-0,00003$ & 0,00009 & $-0,00349$ & 0,00221 & $-0,00119$ & 0,00006 & $-0,00018$ & $-0,00012$ \\
\hline$-\mathrm{F}$ & $-0,00008$ & 0,00076 & $-0,00222$ & 0,00071 & $-0,00075$ & 0,00019 & $-0,00019$ & 0,00000 \\
\hline$-\mathrm{Cl}$ & 0,00005 & 0,00026 & $-0,00164$ & 0,00057 & $-0,00080$ & 0,00000 & $-0,00010$ & $-0,00010$ \\
\hline$-\mathrm{Br}$ & 0,00013 & 0,00010 & $-0,00114$ & 0,00040 & $-0,00063$ & $-0,00006$ & $-0,00005$ & $-0,00011$ \\
\hline$-\mathrm{COH}$ & 0,00025 & $-0,00045$ & $-0,00088$ & 0,00099 & $-0,00035$ & $-0,00027$ & 0,00009 & $-0,00018$ \\
\hline$-\mathrm{COCH}_{3}$ & 0,00020 & $-0,00037$ & $-0,00112$ & 0,00087 & $-0,00062$ & $-0,00019$ & 0,00002 & $-0,00017$ \\
\hline$-\mathrm{COOCH}_{3}$ & 0,00015 & $-0,00025$ & $-0,00065$ & 0,00048 & $-0,00041$ & $-0,00017$ & 0,00003 & $-0,00014$ \\
\hline$-\mathrm{COOH}$ & 0,00014 & $-0,00026$ & $-0,00043$ & 0,00033 & $-0,00036$ & $-0,00018$ & 0,00004 & $-0,00013$ \\
\hline
\end{tabular}




\begin{tabular}{lrrrrrrrr}
\hline Substituinte & $\Delta \rho_{R C P}$ & $\Delta \lambda_{1}$ & $\Delta \lambda_{2}$ & $\Delta \lambda_{3}$ & $\Delta \nabla^{2} \rho_{R C P}$ & $\Delta V_{R C P}$ & $\Delta G_{R C P}$ & $\Delta H_{R C P}$ \\
\hline$-\mathrm{COCl}$ & 0,00016 & $-0,00030$ & $-0,00094$ & 0,00060 & $-0,00064$ & $-0,00016$ & 0,00000 & $-0,00016$ \\
$-\mathrm{CN}$ & $-0,00017$ & 0,00024 & $-0,00097$ & $-0,00032$ & $-0,00106$ & 0,00013 & $-0,00020$ & $-0,00007$ \\
$-\mathrm{NO}_{2}$ & 0,00034 & $-0,00026$ & $-0,00045$ & 0,00093 & 0,00022 & $-0,00035$ & 0,00020 & $-0,00015$ \\
\hline
\end{tabular}

Além disto, as variações da primeira e da terceira curvaturas da densidade eletrônica em relação ao benzeno, $\Delta \lambda_{2}$ e $\Delta \lambda_{3}$, cujos autovetores estão representados na Figura 21, permitem predizer a reatividade e a regiosseletividade dos sistemas estudados. Substituintes ativantes apresentam maiores valores de $\Delta \lambda_{3}$ (entre $2,0 \times 10^{-3}$ e $4,4 \times 10^{-3}$ u. a.) em relação aos desativantes (menores que $1,0 \times 10^{-3}$ u. a.). Por sua vez, grupos orientadores orto/para apresentam $\Delta \lambda_{1}>0$, enquanto o oposto ocorre para os substituintes orientadores meta, que possuem $\Delta \lambda_{1}<0$ (exceto pelo grupo ciano). Por sua vez, as variações na segunda curvatura da densidade eletrônica, $\Delta \lambda_{2}$, tendem a ser mais negativas para substituintes ativantes (de 0,00885 a $-0,00349$ u. a.) do que para os desativantes ( $\geq-0,00222$ u. a.).

Figura 21 - Autovetores das curvaturas da densidade eletrônica $\left(\lambda_{2}\right.$ e $\left.\lambda_{3}\right)$ para o benzeno, $\mathrm{C}_{6} \mathrm{H}_{6}$ plotados com o software Molden (version 5.3) (SCHAFTENAAR e NOORDIK, 2000)

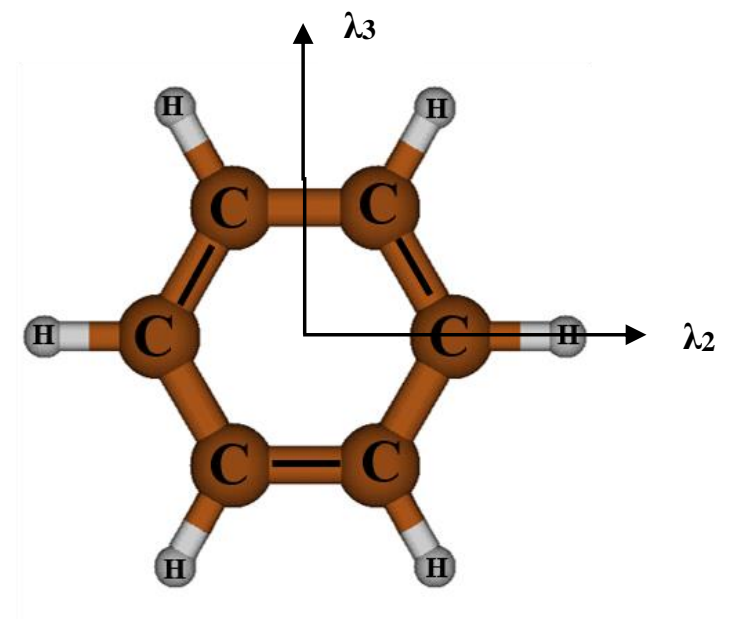

A Figura 22 mostra a correlação entre as variações da primeira e da terceira curvatura da densidade eletrônica, $\Delta \lambda_{1}$ e $\Delta \lambda_{3}$. Como mencionado, os substituintes podem ser separados com base no seu caráter ativante $\left(\Delta \lambda_{3} \geq 0,001\right.$ u. a.) e desativante $\left(\Delta \lambda_{3}<0,001\right.$ u. a.) em reações de substituição eletrofílica. Além disto, observa-se também uma distinção entre estes grupos segundo a sua regiosseletividade, na qual grupos dirigentes orto/para estão situados à direita $\left(\Delta \lambda_{1}>0\right)$ e os dirigentes meta à esquerda $\left(\Delta \lambda_{1}<0\right)$. 
Figura 22 - Relação entre as curvaturas da densidade eletrônica $\Delta \lambda_{1}$ e $\Delta \lambda_{3}$ no ponto crítico do anel, RCP (em u. a.)

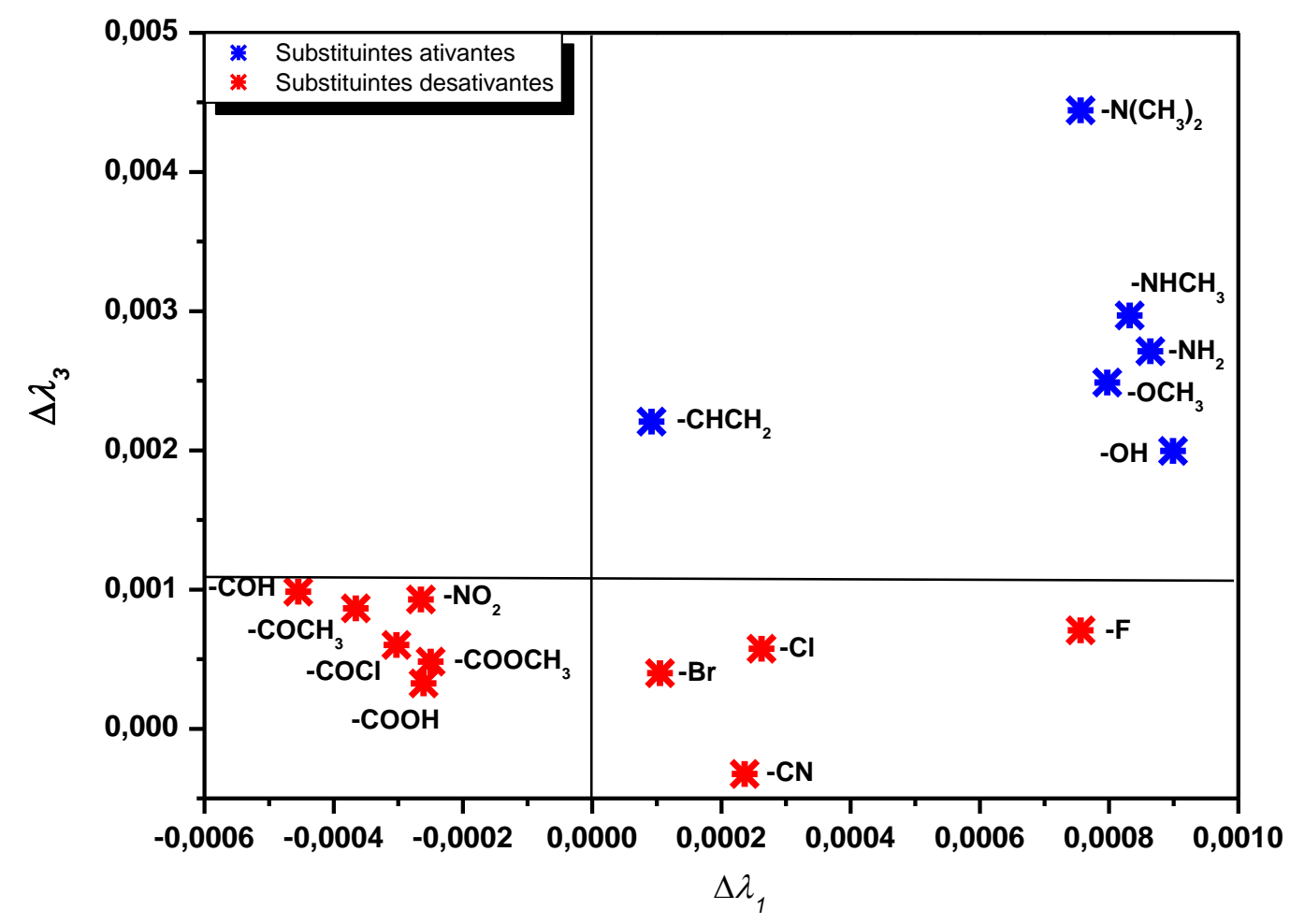

Além disso, há uma correlação linear entre a segunda e a terceira curvatura da densidade eletrônica, $\Delta \lambda_{2}$ e $\Delta \lambda_{3}$, como mostrado na Figura 23, o que está em acordo com o fato destas quantidades serem bons descritores de reatividade, como discutido anteriormente. 
Figura 23 - Correlação entre as curvaturas da densidade eletrônica $\Delta \lambda_{2}$ e $\Delta \lambda_{3}$ no ponto crítico do anel, RCP (em u. a.)

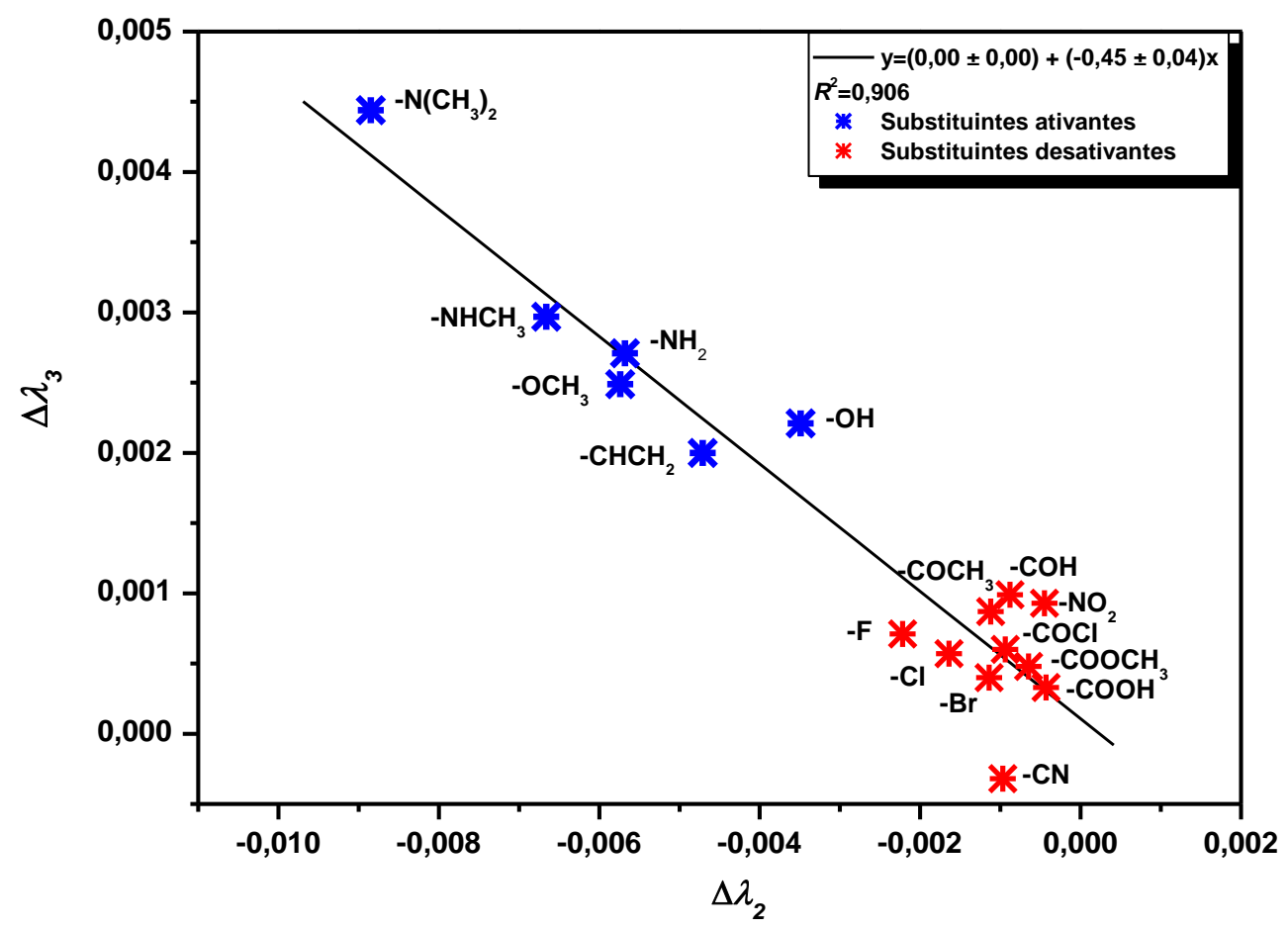

Por sua vez, a correlação entre as variações de densidade de energia cinética e potencial no RCP, em relação ao benzeno, pode ser visualizada na Figura 24. Grupos ativantes apresentam variação de energia potencial sempre positiva $\left(\Delta V_{R C P}>0\right)$, enquanto o oposto é observado para os desativantes $\left(\Delta V_{R C P}<0\right)$, exceto para os substituintes $-\mathrm{F}$ e $-\mathrm{CN}$. Portanto, a energia potencial permite predizer a reatividade de compostos aromáticos substituídos em reações $\mathrm{S}_{\mathrm{E}} \mathrm{Ar}$ a partir das propriedades do substrato inicial. Em contrapartida, a densidade de energia cinética constitui-se como um descritor da regiosseletividade de reações $S_{\mathrm{E}} \mathrm{Ar}$, visto que os substituintes dirigentes orto/para apresentam $\Delta G_{R C P}<0$ e substituintes dirigentes meta possuem $\Delta G_{R C P} \geq 0$ (exceto pelo grupo- $\mathrm{CN}$ ). 
Figura 24 - Correlação entre as variações de densidades de energia cinética $\left(\triangle G_{R C P}\right)$ e potencial $\left(\triangle V_{R C P}\right)$ no ponto crítico do anel em relação ao benzeno (em u. a.)

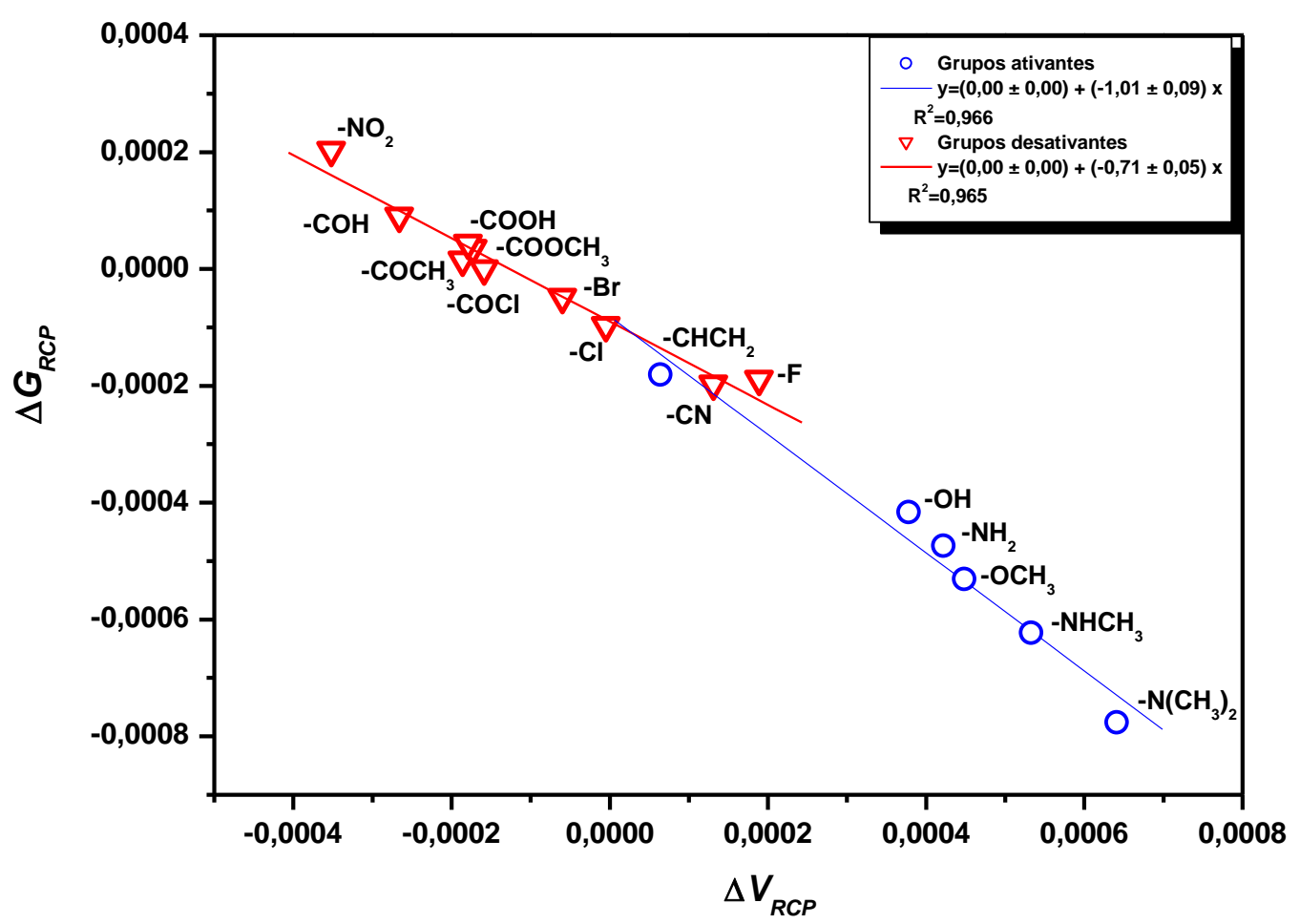

Conforme observado na Figura 24, as correlações entre as densidades de energia potencial e cinética no RCP permitem ainda distinguir os grupos fortemente $\left(-\mathrm{NH}_{2},-\mathrm{NHCH}_{3}\right.$, $-\mathrm{N}\left(\mathrm{CH}_{3}\right)_{2},-\mathrm{OH}$ e $\left.-\mathrm{OCH}_{3}\right)$ daquele fracamente ativante $\left(-\mathrm{CHCH}_{2}\right)$, bem como separar os substituintes fortemente $\left(-\mathrm{NO}_{2}\right)$, moderadamente $\left(-\mathrm{COH},-\mathrm{COCH}_{3},-\mathrm{COOCH}_{3},-\mathrm{COOH}\right.$ e $\mathrm{COCl})$ e fracamente desativantes $(-\mathrm{F},-\mathrm{Cl}$ e $-\mathrm{Br})$, com exceção do grupo ciano $(-\mathrm{CN})$. Isto significa que as correlações observadas nesta figura apresentam algum caráter quantitativo ou, pelo menos, semiquantitativo em termos da reatividade.

Além destas análises, as variações das curvaturas da densidade eletrônica perpendiculares ao anel aromático, nos atratores nucleares dos carbonos, em relação ao benzeno foram avaliadas ( $\Delta \lambda_{\text {perp }}$ ) (Tabela 31$)$. O aumento da população eletrônica $\pi$ resulta em uma concentração maior de carga eletrônica acima e abaixo do plano do anel como mostrado anteriormente pelas análises de quadrupolos atômicos. Portanto, a substituição do hidrogênio por grupos ativantes, orientadores orto/para, fornece valores positivos de $\Delta \lambda_{\text {perp }}$ $\left(61,6\right.$ a 174,1 u. a.) nos átomos de carbono orto/para, e valores negativos de $\Delta \lambda_{\text {perp }}$ nas posições meta. A exceção é $-\mathrm{CHCH}_{2}$, que apresenta um valor positivo de $\Delta \lambda_{\text {perp }}$ apenas na posição para. Todavia, esta propriedade é negativa (-5,1 u. a.), para um dos carbonos na posição orto, o que ainda está em acordo com substituintes dirigentes orto/para porque este valor é maior que aqueles das posições meta. 
Grupos orientadores meta, por sua vez, resultam em valores negativos de $\Delta \lambda_{\text {perp }}$ para a maioria dos carbonos do anel aromático, exceto aquele ligado ao substituinte. No caso do $\mathrm{COH}$, por exemplo, observa-se uma variação da curvatura da densidade eletrônica positiva no carbono meta, indicando incorretamente que esta posição está mais sujeita ao ataque de um possível eletrófilo. Em relação aos halogênios, considerados substituintes orto/para dirigentes, os resultados de $\Delta \lambda_{\text {perp }}$ concordam com os sítios preferenciais de substituição para o flúor e o cloro. Embora para o $-\mathrm{Cl}$ apenas a posição para possua $\Delta \lambda_{\text {perp }}>0$, os carbonos orto ainda apresentam valores menos negativos que os meta. A análise desta propriedade topológica não é tão bem sucedida para o bromo, pois o valor de $\Delta \lambda_{p e r}$ é positivo para o átomo de carbono para, indicando a ativação, e o sítio meta é predito como o mais favorável que o orto. Portanto, as curvaturas da densidade eletrônica perpendiculares ao anel aromático nos atratores nucleares dos átomos de carbono também podem ser empregadas para predizer a reatividade e a regiosseletividade em reações de substituição eletrofílica aromática.

Tabela 31 - Variações das curvaturas da densidade eletrônica perpendiculares ao anel aromático no atratores nucleares de átomos de carbonos, $\Delta \lambda_{\text {perp }}$, de acordo com cálculos B2PLYP/cc-VQZ (em u. a.)

\begin{tabular}{|c|c|c|c|c|c|}
\hline Substituinte & Átomo & $\Delta \lambda_{\text {perp }}{ }^{\mathrm{a}}$ & Substituinte & Átomo & $\Delta \lambda_{\text {perp }}{ }^{\mathrm{a}}$ \\
\hline \multirow[t]{6}{*}{$-\mathrm{NH}_{2}$} & $\mathrm{C}$ & 125,4 & $-\mathrm{Br}$ & $\mathrm{C}$ & $-105,3$ \\
\hline & Corto & 145,3 & & $\mathrm{C}_{\text {Orto }}$ & $-19,1$ \\
\hline & $\mathrm{C}_{\text {Meta }}$ & $-13,8$ & & $\mathrm{C}_{\text {Meta }}$ & $-17,3$ \\
\hline & $\mathrm{C}_{\text {Para }}$ & 124,1 & & $\mathrm{C}_{\text {Para }}$ & 17,0 \\
\hline & $\mathrm{C}_{\text {Meta }}$ & $-13,8$ & & $\mathrm{C}_{\text {Meta }}$ & $-17,3$ \\
\hline & Corto & 145,3 & & Corto & $-19,1$ \\
\hline \multirow[t]{6}{*}{$-\mathrm{NHCH}_{3}$} & $\mathrm{C}$ & 117,4 & $-\mathrm{COH}$ & $\mathrm{C}$ & 12,5 \\
\hline & $\mathrm{C}_{\text {Orto }}$ & 174,1 & & $\mathrm{C}_{\text {Orto }}$ & $-82,7$ \\
\hline & $\mathrm{C}_{\text {Meta }}$ & $-17,2$ & & $\mathrm{C}_{\text {Meta }}$ & 5,2 \\
\hline & $\mathrm{C}_{\text {Para }}$ & 129,4 & & $\mathrm{C}_{\text {Para }}$ & $-71,4$ \\
\hline & $\mathrm{C}_{\text {Meta }}$ & $-13,1$ & & $\mathrm{C}_{\text {Meta }}$ & $-13,0$ \\
\hline & $\mathrm{C}_{\text {Orto }}$ & 135,6 & & $\mathrm{C}_{\text {Orto }}$ & $-91,1$ \\
\hline \multirow[t]{5}{*}{$-\mathrm{N}\left(\mathrm{CH}_{3}\right)_{2}$} & $\mathrm{C}$ & 149,2 & $-\mathrm{COCH}_{3}$ & $\mathrm{C}$ & 26,8 \\
\hline & Corto & 145,7 & & Corto & $-111,8$ \\
\hline & $\mathrm{C}_{\text {Meta }}$ & $-23,2$ & & $\mathrm{C}_{\text {Meta }}$ & $-13,5$ \\
\hline & $\mathrm{C}_{\text {Para }}$ & 121,6 & & $\mathrm{C}_{\text {Para }}$ & $-61,5$ \\
\hline & $\mathrm{C}_{\text {Meta }}$ & $-23,2$ & & $\mathrm{C}_{\text {Meta }}$ & $-0,6$ \\
\hline
\end{tabular}




\begin{tabular}{|c|c|c|c|c|c|}
\hline Substituinte & Átomo & $\Delta \lambda_{\text {perp }}{ }^{\mathrm{a}}$ & Substituinte & Átomo & $\Delta \lambda_{\text {perp }}{ }^{\mathrm{a}}$ \\
\hline & Corto & 145,7 & & Corto & $-68,0$ \\
\hline \multirow[t]{6}{*}{$-\mathrm{OH}$} & $\mathrm{C}$ & 74,1 & $-\mathrm{COOCH}_{3}$ & $\mathrm{C}$ & 109,2 \\
\hline & Corto & 93,9 & & Corto & $-99,7$ \\
\hline & $\mathrm{C}_{\text {Meta }}$ & $-13,7$ & & $\mathrm{C}_{\text {Meta }}$ & $-2,5$ \\
\hline & $\mathrm{C}_{\text {Para }}$ & 103,3 & & $\mathrm{C}_{\text {Para }}$ & $-56,3$ \\
\hline & $\mathrm{C}_{\text {Meta }}$ & $-5,1$ & & $\mathrm{C}_{\text {Meta }}$ & $-1,7$ \\
\hline & $\mathrm{C}_{\text {Orto }}$ & 128,8 & & Corto & $-79,7$ \\
\hline \multirow[t]{6}{*}{$-\mathrm{OCH}_{3}$} & $\mathrm{C}$ & 55,1 & $-\mathrm{COOH}$ & $\mathrm{C}$ & 131,5 \\
\hline & $\mathrm{C}_{\text {Orto }}$ & 61,6 & & $\mathrm{C}_{\text {orto }}$ & $-102,6$ \\
\hline & $\mathrm{C}_{\text {Meta }}$ & $-11,5$ & & $\mathrm{C}_{\text {Meta }}$ & $-2,6$ \\
\hline & $\mathrm{C}_{\text {Para }}$ & 94,9 & & $\mathrm{C}_{\text {Para }}$ & $-63,9$ \\
\hline & $\mathrm{C}_{\text {Meta }}$ & $-4,6$ & & $\mathrm{C}_{\text {Meta }}$ & $-2,3$ \\
\hline & $\mathrm{C}_{\text {orto }}$ & 150,5 & & $\mathrm{C}_{\text {Orto }}$ & $-83,7$ \\
\hline \multirow[t]{5}{*}{$-\mathrm{CHCH}_{2}$} & $\mathrm{C}$ & 111,1 & $-\mathrm{COCl}$ & $\mathrm{C}$ & 118,5 \\
\hline & $\mathrm{C}_{\text {orto }}$ & $-64,4$ & & $\mathrm{C}_{\text {orto }}$ & $-113,5$ \\
\hline & $\mathrm{C}_{\text {Meta }}$ & $-7,9$ & & $\mathrm{C}_{\text {Meta }}$ & $-11,0$ \\
\hline & $\mathrm{C}_{\text {Para }}$ & 1,2 & & $\mathrm{C}_{\text {Para }}$ & $-90,1$ \\
\hline & $\begin{array}{l}\mathrm{C}_{\text {Meta }} \\
\mathrm{C}_{\text {Orto }}\end{array}$ & $\begin{array}{r}-14,6 \\
-5,1\end{array}$ & & $\begin{array}{l}\mathrm{C}_{\text {Meta }} \\
\mathrm{C}_{\text {orto }}\end{array}$ & $\begin{array}{r}-7,3 \\
-119,3\end{array}$ \\
\hline \multirow[t]{6}{*}{$-\mathrm{F}$} & $\mathrm{C}$ & $-13,9$ & $-\mathrm{CN}$ & $\mathrm{C}$ & 312,9 \\
\hline & $\mathrm{C}_{\text {Orto }}$ & 67,3 & & $\mathrm{C}_{\text {orto }}$ & $-57,2$ \\
\hline & $\mathrm{C}_{\text {Meta }}$ & $-1,4$ & & $\mathrm{C}_{\text {Meta }}$ & $-9,9$ \\
\hline & $\mathrm{C}_{\text {Para }}$ & 63,7 & & $\mathrm{C}_{\text {Para }}$ & $-45,4$ \\
\hline & $\mathrm{C}_{\text {Meta }}$ & $-1,4$ & & $\mathrm{C}_{\text {Meta }}$ & $-9,9$ \\
\hline & $\mathrm{C}_{\text {orto }}$ & 67,3 & & Corto & $-57,2$ \\
\hline \multirow[t]{6}{*}{$-\mathrm{Cl}$} & $\mathrm{C}$ & $-36,4$ & $-\mathrm{NO}_{2}$ & $\mathrm{C}$ & $-20,7$ \\
\hline & Corto & $-5,7$ & & Corto & $-66,4$ \\
\hline & $\mathrm{C}_{\text {Meta }}$ & $-12,8$ & & $\mathrm{C}_{\text {Meta }}$ & $-4,5$ \\
\hline & $\mathrm{C}_{\text {Para }}$ & 25,3 & & $\mathrm{C}_{\text {Para }}$ & $-65,6$ \\
\hline & $\mathrm{C}_{\text {Meta }}$ & $-12,8$ & & $\mathrm{C}_{\text {Meta }}$ & $-4,5$ \\
\hline & $\mathrm{C}_{\text {Orto }}$ & $-5,7$ & & $\mathrm{C}_{\text {Orto }}$ & $-66,4$ \\
\hline
\end{tabular}

Além disso, para investigar o caráter quantitativo da reatividade destes novos descritores de reatividade foram obtidas as correlações entre eles e o parâmetro empírico de efeito de ressonância $\left(\sigma_{R}\right)$ de Taft (HANSCH, LEO e TAFT, 1991), comumente utilizado em estudos de reatividade, como mostrado nas Figuras 25 a 26. Os dados de $\sigma_{R}$ para os 
substituintes $-\mathrm{NHCH}_{3},-\mathrm{COOCH}_{3}$ e $-\mathrm{COOH}$ não foram encontrados e, por isso, estes sistemas não aparecem nas figuras mencionadas. $\mathrm{O}$ parâmetro $\sigma_{R}$ é proporcional à diferença do deslocamento do flúor na ressonância magnética nuclear para fluorobenzenos meta e para substituídos, sendo considerado como uma medida empírica da mudança da densidade eletrônica produzida pela presença de um substituinte (HANSCH, LEO e TAFT, 1991; BADER e CHANG, 1989).

Figura 25 - Correlação entre a variação da segunda curvatura da densidade eletrônica, $\Delta \lambda_{2}$, (em u. a.) e o parâmetro empírico de efeito de ressonância $\left(\sigma_{R}\right)$ de Taft (os substituintes ativantes e desativantes estão destacados em azul e vermelho, respectivamente)

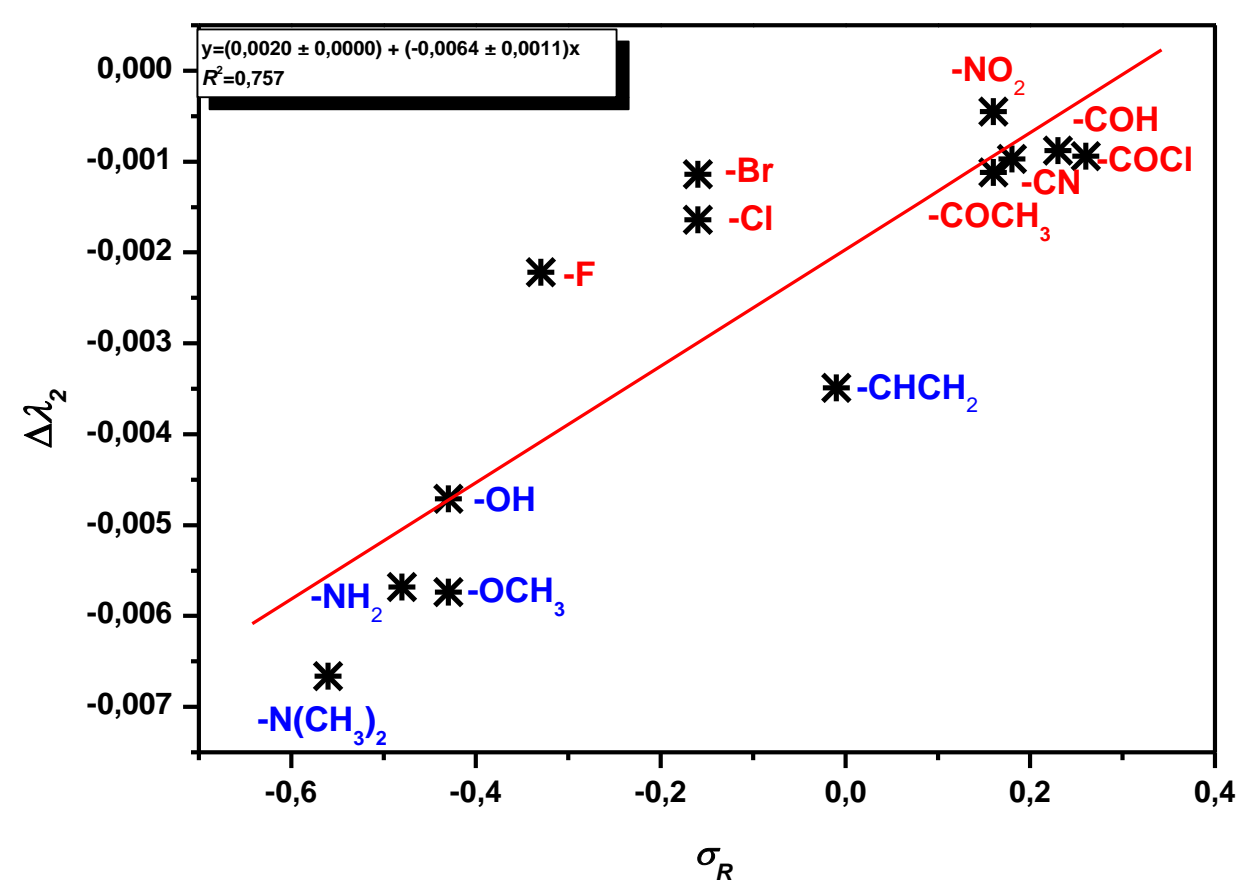


Figura 26 - Correlação entre a variação da densidade de energia potencial em relação ao benzeno, $\Delta V_{R C P}$, (em u. a.) e o parâmetro empírico de efeito de ressonância $\left(\sigma_{R}\right)$ de Taft (os substituintes ativantes e desativantes estão destacados em azul e vermelho, respectivamente)

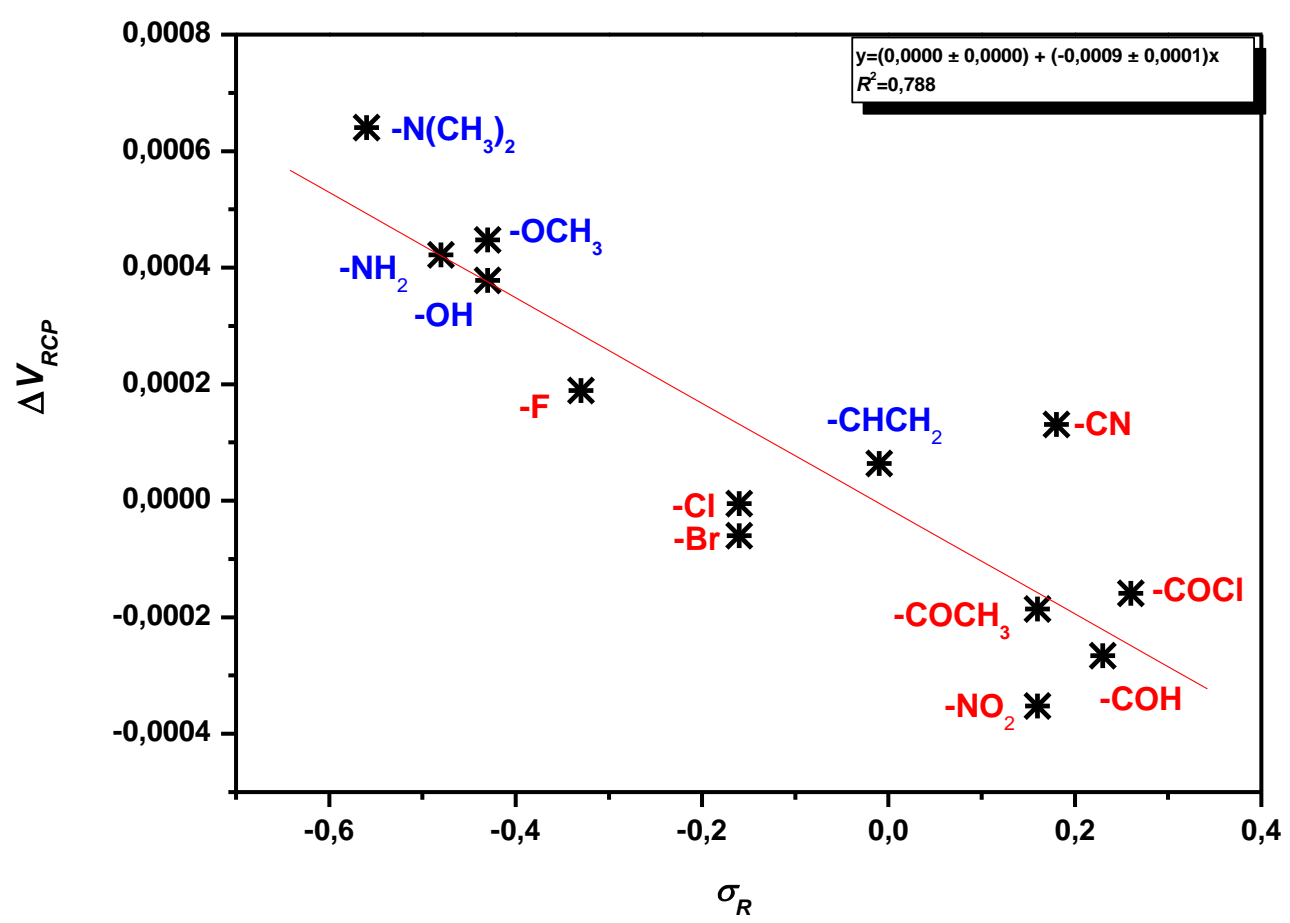

Embora os coeficientes de regressão encontrados não sejam tão elevados (entre 0,681 e 0,788), as correlações apresentadas são animadoras considerando, por exemplo, que os cálculos das curvaturas de $\rho$ e das densidades de energia foram realizados em fase gasosa, enquanto os dados experimentais foram obtidos em diferentes tipos de solventes. Então, os gráficos apresentados mostram uma tendência de separação dos substituintes ativantes dos desativantes, o que está em acordo, de modo geral, com o que foi observado nas Figuras 22 a 24.

As figuras 27 e 28, por sua vez, mostram as correlações entre as variações das curvaturas perpendiculares aos núcleos de carbono orto (valores médios) e para em relação ao benzeno (veja Tabela 31) e o parâmetro empírico de ressonância de Taft (HANSCH, LEO e TAFT, 1991). Os coeficientes de regressão linear são de 0,960 e 0,985, o que evidencia o grande potencial destes novos descritores em estudos de reatividade de compostos aromáticos. 
Figura 27 - Correlação entre a curvatura da densidade eletrônica perpendicular ao núcleo carbono orto em relação ao benzeno, $\Delta \lambda_{\text {perp }}$, (em u. a.) e o parâmetro empírico de ressonância de Taft $\left(\sigma_{R}\right)$ (os substituintes ativantes e desativantes estão destacados em azul, respectivamente)

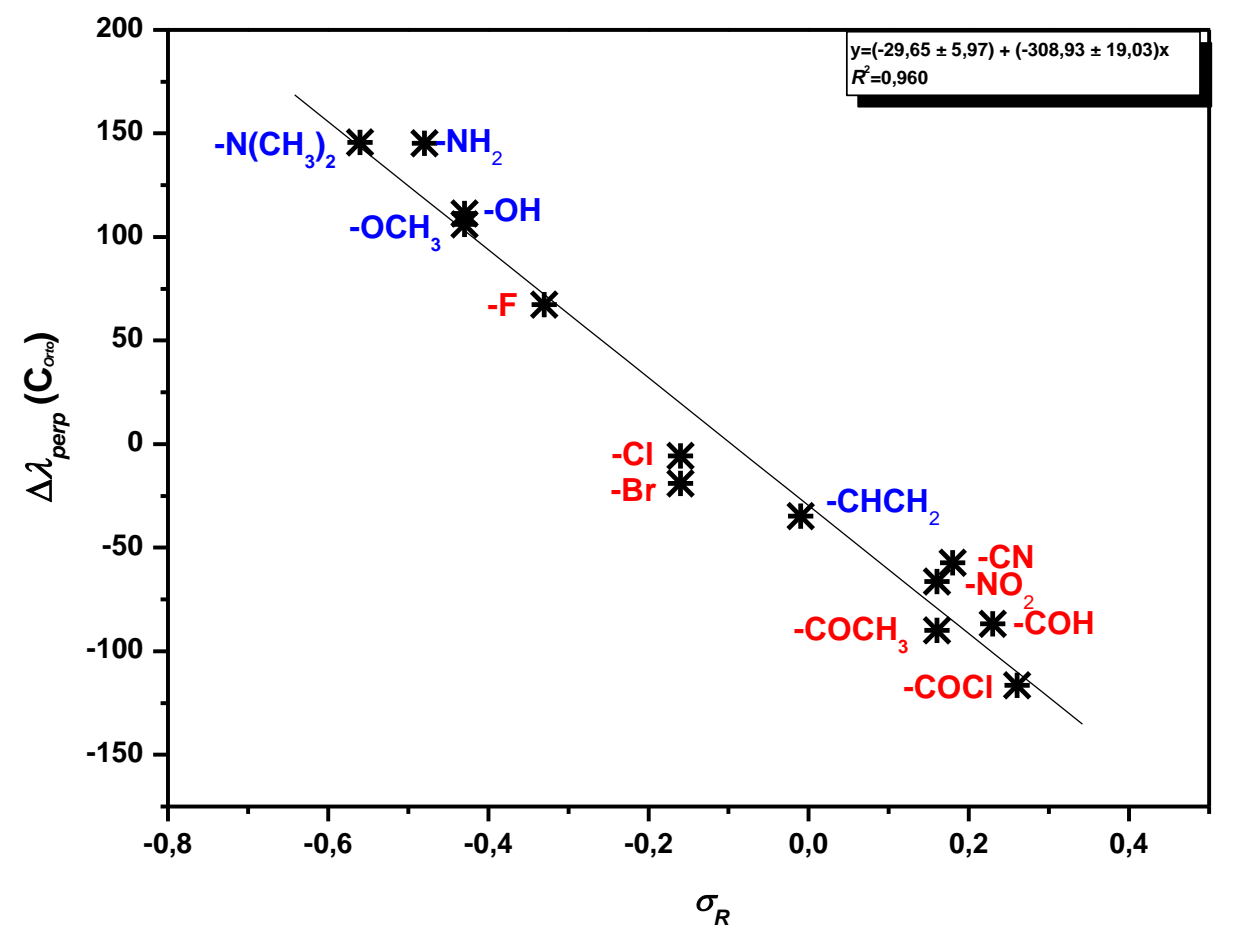

Figura 28 - Correlação entre a curvatura da densidade eletrônica perpendicular ao núcleo carbono para em relação ao benzeno, $\Delta \lambda_{\text {perp }}$, (em u. a.) e o parâmetro empírico de ressonância de Taft $\left(\sigma_{R}\right)$ (os substituintes ativantes e desativantes estão destacados em azul, respectivamente)

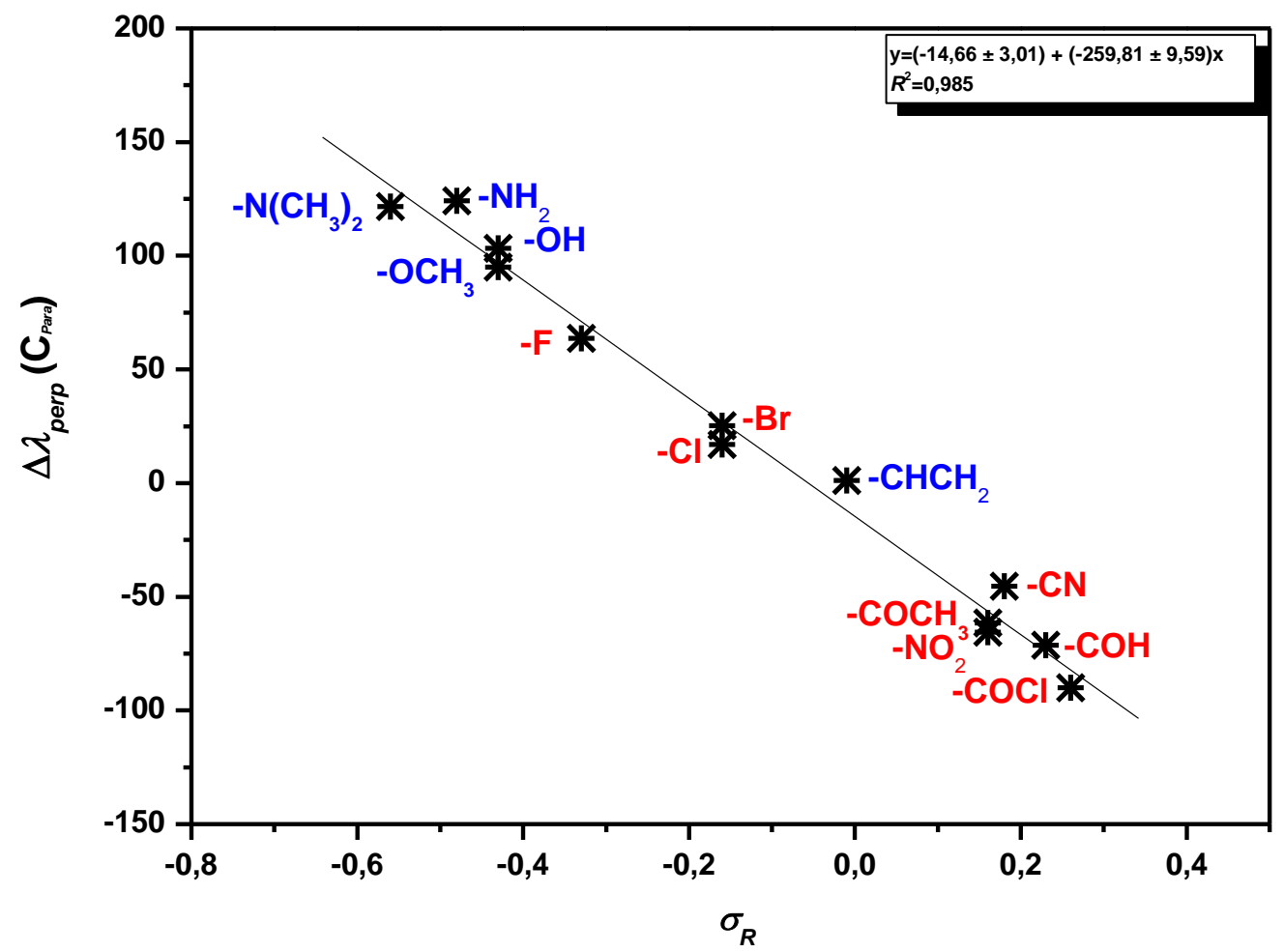




\subsection{Análise das variações de energia intra-atômica segundo a partição de Átomos Quânticos Interagentes}

Com base na partição de Átomos Quânticos Interagentes, proposta por Pendás et al. (2005), avaliou-se a variação da energia intra-atômica durante a substituição do hidrogênio do benzeno por diferentes grupos substituintes $\left(\Delta E_{\text {Intra }}\right)$ como uma possível propriedade preditiva de sítios reativos perante reações de substituição eletrofílica aromática. As maiores variações de energia intra-atômica $(0,020$ a 0,236 u. a.) são correspondentes aos átomos de carbono diretamente ligados aos substituintes (Tabela 32). De modo geral, estas variações são maiores para grupos ativadores do que para grupos desativadores.

Tabela 32 - Variações das energias intra-atômicas e de seus componentes (energia cinética eletrônica do átomo $\Omega, T(\Omega)$, energia de atração elétron-núcleo, $V_{e n}(\Omega)$, e energia de interação elétron-elétron, $V_{e e}(\Omega)$ ) durante a substituição do hidrogênio do benzeno (Ar) por diferentes substituintes (Y), obtidas em nível de cálculo B3LYP/cc-pVQZ (em u. a.)

\begin{tabular}{llrrrr}
\hline Substituinte (Y) & Átomo & $\Delta E_{\text {Intra }}(\Omega)^{\mathrm{a}}$ & $\Delta(T(\Omega))$ & $\Delta\left(V_{\text {ne }}\left(\Omega, \Omega \Omega^{\prime}\right)\right)$ & $\Delta\left(V_{e e}\left(\Omega, \Omega^{\prime}\right)\right)$ \\
\hline$-\mathrm{NH}_{2}$ & $\mathrm{C}$ & 0,137 & $-0,179$ & 1,333 & $-1,017$ \\
& $\mathrm{C}_{\text {Orto }}$ & $-0,001$ & 0,002 & $-0,017$ & 0,014 \\
& $\mathrm{C}_{\text {Meta }}$ & 0,004 & $-0,003$ & 0,023 & $-0,016$ \\
& $\mathrm{C}_{\text {Para }}$ & $-0,003$ & $-0,006$ & 0,001 & 0,001 \\
& $\mathrm{C}_{\text {Meta }}$ & 0,004 & $-0,003$ & 0,023 & $-0,016$ \\
& $\mathrm{C}_{\text {Orto }}$ & $-0,001$ & 0,002 & $-0,017$ & 0,014 \\
\hline$-\mathrm{NHCH}_{3}$ & $\mathrm{C}$ & 0,143 & $-0,185$ & 1,382 & $-1,053$ \\
& $\mathrm{C}_{\text {Orto }}$ & $-0,003$ & $-0,001$ & $-0,032$ & 0,030 \\
& $\mathrm{C}_{\text {Meta }}$ & 0,004 & $-0,004$ & 0,024 & $-0,016$ \\
& $\mathrm{C}_{\text {Para }}$ & $-0,003$ & $-0,005$ & $-0,003$ & 0,005 \\
& $\mathrm{C}_{\text {Meta }}$ & 0,004 & $-0,003$ & 0,022 & $-0,015$ \\
& $\mathrm{C}_{\text {Orto }}$ & $-0,001$ & 0,003 & $-0,027$ & 0,022 \\
\hline$-\mathrm{N}\left(\mathrm{CH}_{3}\right)_{2}$ & $\mathrm{C}_{6}$ & 0,139 & $-0,194$ & 1,379 & $-1,047$ \\
& $\mathrm{C}_{\text {Orto }}$ & $-0,002$ & 0,001 & $-0,037$ & 0,034 \\
& $\mathrm{C}_{\text {Meta }}$ & 0,005 & $-0,001$ & 0,017 & $-0,011$ \\
& $\mathrm{C}_{\text {Para }}$ & $-0,003$ & $-0,002$ & $-0,011$ & 0,010 \\
& $\mathrm{C}_{\text {Meta }}$ & 0,004 & $-0,001$ & 0,016 & $-0,011$ \\
& $\mathrm{C}_{\text {Orto }}$ & $-0,002$ & 0,001 & $-0,037$ & 0,034 \\
\hline & $\mathrm{C}_{6}$ & 0,218 & $-0,277$ & 1,971 & $-1,477$ \\
& $\mathrm{C}_{\text {Orto }}$ & $-0,003$ & 0,003 & 0,021 & $-0,027$ \\
& $\mathrm{C}_{\text {Meta }}$ & 0,003 & $-0,005$ & 0,031 & $-0,023$ \\
& $\mathrm{C}_{\text {Para }}$ & $-0,002$ & $-0,006$ & 0,012 & $-0,008$ \\
& $\mathrm{C}_{\text {Meta }}$ & 0,003 & $-0,006$ & 0,035 & $-0,026$ \\
& $\mathrm{C}_{\text {Orto }}$ & $-0,002$ & 0,007 & $-0,032$ & 0,023 \\
\hline$-\mathrm{OCH}_{3}$ & $\mathrm{C}_{6}$ & 0,218 & $-0,281$ & 1,989 & $-1,490$ \\
& $\mathrm{C}_{\text {Orto }}$ & $-0,003$ & 0,007 & 0,010 & $-0,018$
\end{tabular}




\begin{tabular}{|c|c|c|c|c|c|}
\hline Substituinte (Y) & Átomo & $\Delta E_{\text {Intra }}(\Omega)^{\mathrm{a}}$ & $\Delta(T(\Omega))$ & $\Delta\left(V_{n e}\left(\Omega, \Omega^{\prime}\right)\right)$ & $\Delta\left(V_{e e}\left(\Omega, \Omega^{\prime}\right)\right)$ \\
\hline & $\mathrm{C}_{\text {Meta }}$ & 0,003 & $-0,004$ & 0,025 & $-0,018$ \\
\hline & $\mathrm{C}_{\text {Para }}$ & $-0,002$ & $-0,004$ & 0,005 & $-0,003$ \\
\hline & $\mathrm{C}_{\text {Meta }}$ & 0,003 & $-0,006$ & 0,033 & $-0,024$ \\
\hline & $\mathrm{C}_{\text {orto }}$ & $-0,002$ & 0,004 & $-0,040$ & 0,033 \\
\hline \multirow[t]{6}{*}{$-\mathrm{CHCH}_{2}$} & $\mathrm{C}$ & 0,026 & 0,027 & $-0,132$ & 0,130 \\
\hline & $\mathrm{C}_{\text {orto }}$ & 0,002 & 0,011 & $-0,028$ & 0,019 \\
\hline & $\mathrm{C}_{\text {Meta }}$ & 0,000 & 0,001 & 0,005 & $-0,005$ \\
\hline & $\mathrm{C}_{\text {Para }}$ & $-0,001$ & 0,001 & 0,000 & $-0,002$ \\
\hline & $\mathrm{C}_{\text {Meta }}$ & 0,001 & $-0,001$ & 0,012 & $-0,010$ \\
\hline & $\mathrm{C}_{\text {orto }}$ & 0,003 & 0,007 & $-0,008$ & 0,004 \\
\hline \multirow[t]{6}{*}{$\overline{-F}$} & $\mathrm{C}$ & 0,236 & $-0,294$ & 2,075 & $-1,545$ \\
\hline & $\mathrm{C}_{\text {orto }}$ & $-0,003$ & 0,005 & 0,035 & $-0,043$ \\
\hline & $\mathrm{C}_{\text {Meta }}$ & 0,002 & $-0,008$ & 0,043 & $-0,033$ \\
\hline & $\mathrm{C}_{\text {Para }}$ & $-0,002$ & $-0,006$ & 0,023 & $-0,018$ \\
\hline & $\mathrm{C}_{\text {Meta }}$ & 0,002 & $-0,008$ & 0,043 & $-0,033$ \\
\hline & $\mathrm{C}_{\text {orto }}$ & $-0,003$ & 0,005 & 0,035 & $-0,043$ \\
\hline \multirow[t]{6}{*}{$\overline{-\mathrm{Cl}}$} & $\mathrm{C}$ & 0,060 & $-0,040$ & 0,257 & $-0,158$ \\
\hline & $\mathrm{C}_{\text {orto }}$ & 0,002 & $-0,003$ & 0,085 & $-0,079$ \\
\hline & $\mathrm{C}_{\text {Meta }}$ & 0,001 & $-0,004$ & 0,031 & $-0,026$ \\
\hline & $\mathrm{C}_{\text {Para }}$ & $-0,001$ & $-0,003$ & 0,020 & $-0,018$ \\
\hline & $\mathrm{C}_{\text {Meta }}$ & 0,001 & $-0,004$ & 0,030 & $-0,026$ \\
\hline & $\mathrm{C}_{\text {Orto }}$ & 0,002 & $-0,003$ & 0,085 & $-0,079$ \\
\hline \multirow[t]{6}{*}{$-\mathrm{Br}$} & $\mathrm{C}$ & 0,043 & 0,014 & $-0,216$ & 0,246 \\
\hline & $\mathrm{C}_{\text {orto }}$ & 0,003 & $-0,005$ & 0,092 & $-0,084$ \\
\hline & $\mathrm{C}_{\text {Meta }}$ & 0,001 & $-0,003$ & 0,029 & $-0,025$ \\
\hline & $\mathrm{C}_{\text {Para }}$ & $-0,001$ & $-0,003$ & 0,020 & $-0,018$ \\
\hline & $\mathrm{C}_{\text {Meta }}$ & 0,000 & $-0,003$ & 0,028 & $-0,025$ \\
\hline & $\mathrm{C}_{\text {orto }}$ & 0,003 & $-0,005$ & 0,092 & $-0,084$ \\
\hline \multirow[t]{6}{*}{$-\mathrm{COH}$} & $\mathrm{C}$ & 0,021 & 0,079 & $-0,308$ & 0,249 \\
\hline & $\mathrm{C}_{\text {orto }}$ & 0,003 & 0,014 & $-0,036$ & 0,025 \\
\hline & $\mathrm{C}_{\text {Meta }}$ & $-0,001$ & $-0,004$ & 0,028 & $-0,025$ \\
\hline & $\mathrm{C}_{\text {Para }}$ & 0,002 & 0,002 & 0,015 & $-0,014$ \\
\hline & $\mathrm{C}_{\text {Meta }}$ & $-0,001$ & $-0,004$ & 0,028 & $-0,026$ \\
\hline & $\mathrm{C}_{\text {orto }}$ & 0,002 & 0,011 & 0,006 & $-0,014$ \\
\hline \multirow[t]{6}{*}{$-\mathrm{COCH}_{3}$} & $\mathrm{C}$ & 0,020 & 0,065 & $-0,287$ & 0,242 \\
\hline & $\mathrm{C}_{\text {orto }}$ & 0,003 & 0,015 & $-0,006$ & $-0,006$ \\
\hline & $\mathrm{C}_{\text {Meta }}$ & $-0,001$ & $-0,001$ & 0,018 & $-0,018$ \\
\hline & $\mathrm{C}_{\text {Para }}$ & 0,002 & 0,003 & 0,006 & $-0,007$ \\
\hline & $\mathrm{C}_{\text {Meta }}$ & 0,000 & $-0,001$ & 0,015 & $-0,014$ \\
\hline & $\mathrm{C}_{\text {Orto }}$ & 0,004 & 0,012 & $-0,035$ & 0,027 \\
\hline \multirow[t]{6}{*}{$-\mathrm{COOCH}_{3}$} & $\mathrm{C}$ & 0,021 & 0,052 & $-0,205$ & 0,175 \\
\hline & $\mathrm{C}_{\text {orto }}$ & 0,003 & 0,012 & 0,005 & $-0,014$ \\
\hline & $\mathrm{C}_{\text {Meta }}$ & 0,000 & $-0,002$ & 0,019 & $-0,017$ \\
\hline & $\mathrm{C}_{\text {Para }}$ & 0,002 & 0,003 & 0,006 & $-0,007$ \\
\hline & $\mathrm{C}_{\text {Meta }}$ & 0,000 & $-0,001$ & 0,015 & $-0,015$ \\
\hline & $\mathrm{C}_{\text {orto }}$ & 0,003 & 0,007 & 0,011 & $-0,015$ \\
\hline
\end{tabular}




\begin{tabular}{|c|c|c|c|c|c|}
\hline Substituinte (Y) & Átomo & $\Delta E_{\text {Intra }}(\Omega)^{\mathrm{a}}$ & $\Delta(T(\Omega))$ & $\Delta\left(V_{n e}\left(\Omega, \Omega^{\prime}\right)\right)$ & $\Delta\left(V_{e e}\left(\Omega, \Omega^{\prime}\right)\right)$ \\
\hline \multirow[t]{6}{*}{$-\mathrm{COOH}$} & $\mathrm{C}$ & 0,020 & 0,052 & $-0,202$ & 0,171 \\
\hline & $\mathrm{C}_{\text {Orto }}$ & 0,003 & 0,012 & 0,010 & $-0,018$ \\
\hline & $\mathrm{C}_{\text {Meta }}$ & $-0,001$ & $-0,002$ & 0,022 & $-0,021$ \\
\hline & $\mathrm{C}_{\text {Para }}$ & 0,002 & 0,003 & 0,008 & $-0,010$ \\
\hline & $\mathrm{C}_{\text {Meta }}$ & $-0,001$ & $-0,002$ & 0,019 & $-0,018$ \\
\hline & $\mathrm{C}_{\text {orto }}$ & 0,003 & 0,008 & 0,013 & $-0,017$ \\
\hline \multirow[t]{6}{*}{$-\mathrm{COCl}$} & $\mathrm{C}$ & 0,022 & 0,039 & $-0,143$ & 0,126 \\
\hline & $\mathrm{C}_{\text {orto }}$ & 0,004 & 0,010 & 0,023 & $-0,029$ \\
\hline & $\mathrm{C}_{\text {Meta }}$ & $-0,001$ & $-0,002$ & 0,032 & $-0,031$ \\
\hline & $\mathrm{C}_{\text {Para }}$ & 0,002 & 0,004 & 0,017 & $-0,019$ \\
\hline & $\mathrm{C}_{\text {Meta }}$ & $-0,001$ & $-0,003$ & 0,032 & $-0,030$ \\
\hline & $\mathrm{C}_{\text {Orto }}$ & 0,003 & 0,010 & 0,019 & $-0,026$ \\
\hline \multirow[t]{6}{*}{$-\mathrm{CN}$} & $\mathrm{C}$ & 0,029 & $-0,018$ & 0,124 & $-0,077$ \\
\hline & $\mathrm{C}_{\text {orto }}$ & 0,003 & $-0,003$ & 0,071 & $-0,064$ \\
\hline & $\mathrm{C}_{\text {Meta }}$ & $-0,001$ & $-0,003$ & 0,035 & $-0,033$ \\
\hline & $\mathrm{C}_{\text {Para }}$ & 0,001 & 0,000 & 0,025 & $-0,024$ \\
\hline & $\mathrm{C}_{\text {Meta }}$ & $-0,001$ & $-0,003$ & 0,035 & $-0,033$ \\
\hline & $\mathrm{C}_{\text {Orto }}$ & 0,003 & $-0,003$ & 0,071 & $-0,064$ \\
\hline \multirow[t]{6}{*}{$-\mathrm{NO}_{2}$} & $\mathrm{C}$ & 0,090 & $-0,058$ & 0,695 & $-0,548$ \\
\hline & $\mathrm{C}_{\text {orto }}$ & 0,002 & 0,007 & 0,062 & $-0,067$ \\
\hline & $\mathrm{C}_{\text {Meta }}$ & $-0,001$ & $-0,007$ & 0,051 & $-0,045$ \\
\hline & $\mathrm{C}_{\text {Para }}$ & 0,001 & 0,001 & 0,027 & $-0,026$ \\
\hline & $\mathrm{C}_{\text {Meta }}$ & $-0,001$ & $-0,007$ & 0,051 & $-0,045$ \\
\hline & $\mathrm{C}_{\text {Orto }}$ & 0,003 & 0,007 & 0,063 & $-0,067$ \\
\hline
\end{tabular}

${ }^{\mathrm{a}} \Delta E_{\text {Intra }}(\Omega)=E_{\text {Intra }}(\Omega)_{\text {ArY }}-E_{\text {Intra }}(\Omega)_{\text {Ar }}$, com definições análogas para os termos nas demais colunas.

É possível observar que, aparentemente, a posição de ataque mais favorável está associada a valores menores de $\Delta E_{\text {Intra }}$ Os grupos $-\mathrm{NH}_{2},-\mathrm{NHCH}_{3},-\mathrm{N}\left(\mathrm{CH}_{3}\right)_{2},-\mathrm{OH},-\mathrm{OCH}_{3} \mathrm{e}$ -F, considerados experimentalmente orientadores orto/para, acarretam uma variação negativa da energia intra-atômica nos carbonos nestas posições (de $-0,001$ a $-0,003$ u. a.), enquanto $\Delta E_{\text {Intra }}$ é positivo para os carbonos das posições meta destes sistemas. Porém, esta quantidade falha parcialmente ao tratar o substituinte ativante $-\mathrm{CHCH}_{2}$. Neste caso, segundo esta análise energética, apenas a posições para é prevista como suscetível a reações $\mathrm{S}_{\mathrm{E}} \mathrm{Ar}$, com uma variação da energia intra-atômica de -0,001 u. a. em relação benzeno, ou seja, valores positivos de $\Delta E_{\text {Intra }}$ são obtidos para as posições orto e meta, com inversão da tendência esperada nestes carbonos. Em relação aos halogênios, as posições orto e para do flúor são as mais suscetíveis ao ataque de um eletrófilo, enquanto para o cloro e bromo, a energia intraatômica prediz que sítio para é o mais reativo. 
Por outro lado, os substituintes mais desativadores que os halogênios, exceto o $\mathrm{COOCH}_{3}$, causam uma variação negativa, em torno de $-0,001$ u. a., da energia intra-atômica nos carbonos meta, enquanto os carbonos das posições orto e para apresentam $\Delta E_{\text {Intra }}$ positivo. Este descritor consegue prever a posição correta mesmo para o substituinte $\mathrm{COOCH}_{3}$, pois valores mais positivos são encontrados para $\Delta E_{\text {Intra }}$ dos carbonos orto e para do que para a posição meta. A energia intra-atômica, assim como o momento de quadrupolo atômico, não permite fazer previsões sobre as porcentagens de isômeros orto, meta e para esperadas para reações de $S_{E} A r$, constituindo-se apenas como uma ferramenta qualitativa promissora para a determinação dos possíveis sítios reativos.

Os valores dos componentes da energia intra-atômica (energia cinética eletrônica, energia de atração entre a densidade eletrônica do átomo e seu núcleo e energia de interação elétron-elétron) não estão tão correlacionados com os processos de ativação e desativação do anel aromático e com o favorecimento das posições orto, meta e para durante a troca de substituintes.

\section{CONCLUSÕES}

De modo geral, grupos ativadores tendem a gerar variações negativas de $Q_{z z}$ em certos átomos de carbono, com relação ao benzeno $\left(\Delta Q_{z z}\right)$, que correspondem a distorções da nuvem eletrônica para fora do plano do anel, enquanto grupos desativadores tendem a ocasionar o fenômeno oposto, ou seja, variações positivas de $Q_{z z}$ em todos os átomos de carbono do anel. Verificamos também, como previamente discutido na literatura, que as posições com os menores valores de $\Delta Q_{z z}$ são aquelas mais favoráveis a um ataque eletrofílico. Por sua vez, os valores das variações das curvaturas da densidade eletrônica e das densidades de energia cinética e potencial nos RCPs, em relação aos dados do benzeno, permitem predizer tanto a reatividade, quanto a regiosseletividade em reações de substituição eletrofílica aromática. As duas últimas quantidades possibilitam, ainda, separar os diferentes substituintes de acordo com o grau de ativação e desativação. As curvaturas da densidade eletrônica perpendiculares ao anel aromático nos atratores nucleares de átomos de carbono também podem ser considerados descritores promissores nos estudos de reatividade e regiosseletividade destes compostos. Por fim, notamos que a energia intra-atômica, obtida a partir da análise de Átomos Quânticos Interagentes (IQA), configura-se como uma quantidade alternativa para a predição de sítios reativos frente a reações de $S_{E} A r$. 


\section{PERSPECTIVAS}

Utilizar descritores como quadrupolos atômicos e densidades de energia cinética e potencial nos RCPs para predizer a ordem de reatividade dos grupos $(\mathrm{R}),-\mathrm{NO}_{2},-\mathrm{Br},-\mathrm{Cl},-$ $\mathrm{COOH},-\mathrm{H},-\mathrm{F},-\mathrm{C}\left(\mathrm{CH}_{3}\right)_{3},-\mathrm{CH}_{3},-\mathrm{OCH}_{3},-\mathrm{OC}_{2} \mathrm{H}_{5},-\mathrm{N}\left(\mathrm{CH}_{3}\right)_{2},-\mathrm{OH}$ e $-\mathrm{NH}_{2}$, em reações de substituição nucleofílica aromática.

\section{REFERÊNCIAS}

BADER, R. F. W. Atoms in Molecules: A Quantum Theory. $1^{\text {a }}$ ed. Oxford, UK: Clarendon Press, 1990.

BADER, R. F. W. e CHANG, C. Properties of atoms in molecules: electrophilic aromatic substitution. The Journal of Physical Chemistry, v. 93, p. 2946-2956, 1989.

BAUZÁ, A.; QUIÑONERO, D.; DEYÀ, P. M. e FRONTERA, A. Quadrupole moment versus molecular electrostatic potential: strange behavior of ethynyl-substituted benzenes. Chemical Physics Letters, v. 567, p. 60-65, 2013.

BLANCO, M. A.; PENDÁS, A. M. e FRANCISCO, E. Interacting quantum atoms: a correlated energy decomposition scheme based on the quantum theory of atoms in molecules. Journal of Chemical Theory and Computation, v. 1, p. 1096-1109, 2005.

BRUICE, P. Y. Organic Chemistry. Upper Saddle River: Prentice Hall, 2004.

CLAIDEN, J.; GREEVES, N. e WARREN, S. Organic Chemistry, 2a ed., Oxford: Oxford University Press, 2012.

CORMIER, K. W.; WATT, M. e LEWIS, M. The quadrupole moment of substituted cyclopentadienyl anion. The Journal of Physical Chemistry A, v. 114, p. 11708-11713, 2010.

DUNNING JR, T. H. Gaussian basis sets for use in correlated molecular calculations. I. The atoms boron through neon and hydrogen. The Journal of Chemical Physics, v. 90, p. 1007$1023,1989$.

FIEVEZ, T.; PINTER, B.; GEERLINGS, P.; BICKELHAUPT, F. M. e De PROFT, F., Regioselectivity in electrophilic aromatic substitution: insights from interaction energy decomposition potentials. European Journal of Organic Chemistry, v.16, p. 2958-2968, 2011.

FRISCH, M. J.; TRUCKS G. W.; SCHLEGEL, H. B.; SCUSERIA, G. E.; ROBB, M. A.; CHEESEMAN, J. R.; SCALMANI, G.; BARONE, V.; MENNUCCI, B.; PETERSSON, G. A.; NAKATSUJI, H.; CARICATO, M.; LI, X.; HRATCHIAN, H. P.; IZMAYLOV, A. F.; 
BLOINO, J.; ZHENG, G.; SONNENBERG, J. L.; HADA, M.; EHARA, M.; TOYOTA, K.; FUKUDA, R.; HASEGAWA, J.; ISHIDA, M.; NAKAJIMA, T.; HONDA, Y.; KITAO, O.; NAKAI, H.; VREVEN, T.; MONTGOMERY JR., J. A.; PERALTA, J. E.; OGLIARO, F.; BEARPARK, M.; HEYD, J. J.; BROTHERS, E.; KUDIN, K. N.; STAROVEROV, V. N.; KEITH, T.; KOBAYASHI, R.; NORMAND, J.; RAGHAVACHARI, K.; RENDELL, A.; BURANT, J. C.; IYENGAR, S. S.; TOMASI, J.; COSSI, M.; REGA, N.; MILLAM, J. M.; KLENE, M.; KNOX, J. E.; CROSS, J. B.; BAKKEN, V.; ADAMO, C.; JARAMILLO, J.; GOMPERTS, R.; STRATMANN, R. E.; YAZYEV, O.; AUSTIN, A. J.; CAMMI, R.; POMELLI, C.; OCHTERSKI, J. W.; MARTIN, R. L.; MOROKUMA, K.; ZAKRZEWSKI, V. G.; VOTH, G. A.; SALVADOR, P.; DANNENBERG, J. J.; DAPPRICH, S.; DANIELS, A. D.; FARKAS, O.; FORESMAN, J. B.; ORTIZ, J. V.; CIOSLOWSKI, J. e FOX, D. J. Gaussian 09, Revision D.01, Gaussian Inc., Wallingford, CT, 2013.

GRIMME, S. Semiempirical hybrid density functional with perturbative second-order correlation. The Journal of Chemical Physics, v. 124, 034108, 2006.

HANSCH, C; LEO, A. e TAFT, R. W. A Survey of Hammett substituent constants and resonance and field parameters. Chemical Review, v. 91, p. 165-195, 1991.

KEITH, T. A. AIMAll (version 16.10.31), TK Gristmill Software, Overland Park KS, USA, 2013.

KOLEVA, G.; GALABOV, B.; WU, J. I.; SCHAEFER III, H. F. e SCHLEYER, P, von R. Electrophile affinity: a reactivity measure for aromatic substitution. Journal of the American Chemical Society, v. 131, p. 14722-14727, 2009.

LIU, S. Where does the electron go? The nature of ortho/para and meta group directing in electrophilic aromatic substitution, The Journal of Chemical Physics, v. 141, 10.1063/1.4901898, 2014

MATTA, C. F. e BOYD, R. J. The Quantum Theory of Atoms in Molecules From Solid State to DNA and Drug Design, Weinheim: Wiley-Vch, 2007.

McMURRY, J. Organic Chemistry. 7ª ed., Massachusetts: Brooks Cole, 2007.

MOA, G. M. J. e MOSQUERA, R. A. On the electron donor and the electrophilic substitution activating abilities of substituents in uracil. The Journal Physical Chemistry A, v. 110, p. 5934-5941, 2006.

PENDÁS, A. M.; BLANCO, M. A. e FRANCISCO, E. The nature of the hydrogen bond: a synthesis from the interacting quantum atoms picture. The Journal of Chemical Physics, v. $125,184112,2006$.

POLITZER, P.; ABRAHMSEN, L. e SJOBERG, P. Effects of amino and nitro substituents upon the electrostatic potential of an aromatic ring. Journal of the American Chemical Society. v. 106, p. 855-860, 1984.

SCHAFTENAAR, G. e NOORDIK J. H. Molden: a pre- and post-processing program for molecular and electronic structures, Journal Computational - Aided Molecular Design, v. 14, p. 123-134, 2000. 
WILSON, A. K.; WOON, D. E.; PETERSON, K. A. e DUNNING JR, T. H. Gaussian basis sets for use in correlated molecular calculations. IX. The atoms gallium through krypton. Journal of Chemical Physics, v. 110, 7667-7676, 1999.

WOON, D. E. e DUNNING Jr., T. H. Gaussian-basis sets for use in correlated molecular calculations. 3. The atoms aluminum through argon. The Journal of Chemical Physics, v. 98, p. 1358-1371, 1993.

ZHOU, Z. e PARR, R. G. Activation hardness: new index for describing the orientation of electrophilic aromatic substitution. Journal of the American Chemical Society. v. 112, 5720-5724, 1990.

\section{CONSIDERAÇÕES FINAIS}

A formação de ligações de hidrogênio durante a complexação de ácidos carboxílicos, a coordenação dos ligantes carbonil e cianeto a centros metálicos distintos e a excitação eletrônica de moléculas diatômicas simples provocam variações significativas nas intensidades de infravermelho de certos modos vibracionais. Para compreender a razão destas mudanças, esta propriedade espectroscópica foi decomposta em contribuições de carga, fluxo de carga e fluxo de dipolo de acordo com o preconizado pelo modelo CCFDF/QTAIM. Para os primeiros dois casos, as mudanças nesta propriedade espectroscópica podem ser justificadas pela transferência significativa de carga eletrônica que reforça as derivadas do momento de dipolo devido ao deslocamento dos átomos carregados (termo cruzado entre carga e fluxo de carga). Por sua vez, no que se refere ao processo de excitação eletrônica, observa-se que a soma de três contribuições é necessária para explicar as variações de intensidade observadas, carga e os termos cruzados entre a carga com fluxo de carga e fluxo de dipolo.

As transições eletrônicas podem provocar inversões no sentido do vetor de momento de dipolo, como ocorre nas excitações $\sigma \rightarrow \pi^{*}$ para o estado tripleto das moléculas $\mathrm{BF}, \mathrm{BCl}$, $\mathrm{BBr}$ e CS. Isto ocorre em virtude da diminuição expressiva das contribuições de polarização atômica do boro e do carbono durante a excitação.

Por fim, as propriedades tais como as densidades de energia cinética e potencial, as curvaturas das densidades eletrônicas nos pontos críticos dos anéis aromáticos e dos núcleos dos átomos de carbono, assim como a energia intra-atômica, são apresentadas como descritores alternativos para predizer a reatividade e a regiosseletividade em reações de substituição eletrofílica aromática baseando-se apenas nas características dos substratos iniciais. 